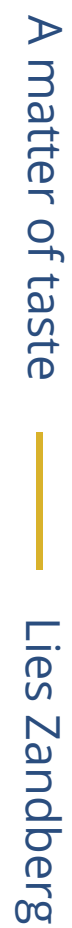

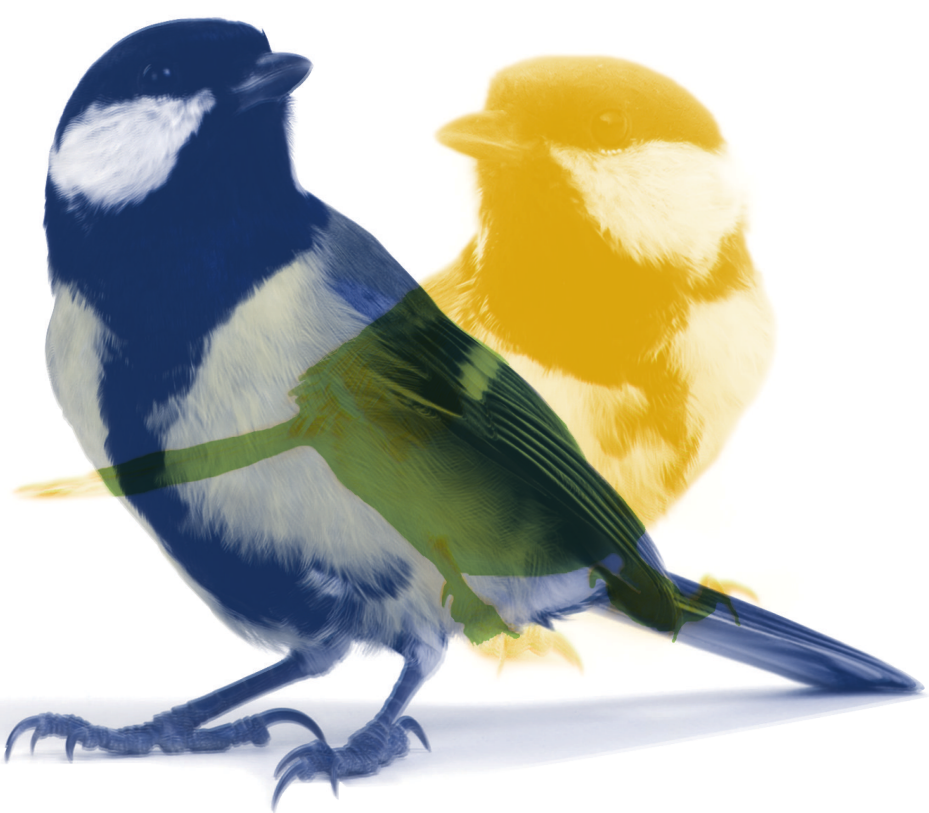

\title{
A matter of taste
}




\section{A matter of taste}

The role of compatibility in mate preferences in great tits

Lies Zandberg 


\section{Thesis committee}

\section{Promotor}

Prof. Dr Marc Naguib

Professor of Behavioural Ecology

Wageningen University \& Research

\section{Co-promotors}

Dr Camilla Hinde

Associate Professor, Behavioural Ecology Group

Wageningen University \& Research

Prof. Dr Kees van Oers

Extraordinary Professor of Animal personality

Wageningen University \& Research / Netherlands Institute of Ecology (NIOO-KNAW), Wageningen

\section{Other members}

Prof. Dr Trond Amundsen - Norwegian University of Science and Technology, Norway Prof. Dr Martien A.M. Groenen - Wageningen University \& Research

Dr Nick J. Royle - University of Exeter, United Kingdom

Dr Martine E. Maan - University of Groningen, Netherlands

This research was conducted under the auspices of the Graduate School Wageningen Institute of Animal Sciences. 


\section{A matter of taste}

The role of compatibility in mate preferences in great tits

Elisabeth Christina Ada (Lies) Zandberg

Thesis

submitted in fulfilment of the requirements for the degree of doctor

at Wageningen University

by the authority of the Rector Magnificus,

Prof. Dr A.P.J. Mol,

in the presence of the

Thesis Committee appointed by the Academic Board

to be defended in public on Friday 2 November 2018

at $4 \mathrm{pm}$ in the Aula. 
Lies Zandberg

A matter of taste: The role of compatibility in mate preferences in great tits 235 pages

PhD thesis, Wageningen University, Wageningen, the Netherlands (2018)

With references, with summaries in English and Dutch

ISBN 978-94-6343-516-1

DOI https://doi.org/10.18174/459842 
"Beauty is no quality in things themselves: It exists merely in the mind which contemplates them; and each mind perceives a different beauty."

David Hume, 1757 



\section{Table of Contents}

Chapter $1 \quad$ General Introduction 9

Chapter 2 Measuring mate preferences: Absolute versus comparative 33 evaluation of potential partners

Chapter 3 Direct fitness benefits explain mate preference, but not choice, for 57 similarity in heterozygosity levels

Chapter 4 Estimating individual mate attractiveness and the reproductive benefits of compatibility

Chapter 5 Provisioning is affected by personality, but not the compatibility of personalities in great tit parents

Chapter 6 General Discussion

References

Summary

Samenvatting

Curriculum Vitae

Acknowledgements

WIAS Training and Education statement 

Chapter 1

General introduction 
Tn 1860 Charles Darwin wrote: "The sight of a feather in a peacock's tail, whenever I gaze at it, 1 makes me sick!". When considering his theory of natural selection, it seemed counterintuitive that animals would have evolved such exaggerated traits that do not aid, and sometimes even hinder, their survival abilities. Some years later Darwin himself proposed a theory that would explain the evolution and the maintenance of these traits. Sexual selection "depends on the advantage which certain individuals have over other individuals of the same sex and species, in exclusive relation to reproduction." (Darwin 1871). Under sexual selection traits are selected to increase an individuals' reproductive success through competition over mates: it is selection generated by differential access to gametes of the opposite sex allowing the bearer of those traits to leave more copies of its alleles in future generations (Andersson 1994; Kokko et al. 2006).

Typically, sexual selection is divided into intra- and intersexual selection, leading to the evolution of armaments and ornaments (Danchin and Cézilly 2008). In the first case individuals compete with others of the same sex for access to the gametes of the opposite sex. This competition is usually most intense in the sex that has greater variance in reproductive success (Bateman 1948). Traits have evolved to increase success in these contests, such as scramble competition or sperm competition. In the second case traits have evolved to attract or stimulate potential mates (Andersson 1994). In the broad sense this can also include passive processes that increase the likelihood of individuals of one sex to be encountered or found by the other sex. However, in the narrow sense, intersexual selection describes the selection of certain phenotypes or traits of one sex by the other (Andersson 1994). Individuals that have these traits are preferred over other individuals not possessing these traits (see Andersson and Iwasa 1996; Rosenthal 2017). By having preferences for certain traits in a mate, individuals can gain benefits through which they can increase their reproductive success and with it their fitness.

\section{Terminology}

While often females are considered as the choosing, and males as the courting sex, in more and more species it is found that there is no such clear division. In some species females choose, in others males are the choosier sex, and in many other species there is mutual mate choice (Amundsen 2000; Danchin and Cézilly 2008). Therefore, following Rosenthal (2017), I will use the terms chooser and courter.

The process of mate choice can be defined as the effects of traits in one sex that lead to non-random mating or non-random allocation of reproductive investment with members of the opposite sex (Kokko et al. 2003; Edward 2015). Mate preferences, on the other hand, say something about a chooser's internal representation of courter traits that predisposes her (or him) to mate with some phenotypes over others (Jennions and Petrie 1997). Thus, mate choice is the outcome of the interaction between preferences and other internal and 
external factors such as competition over mates and the availability of mates. Therefore, throughout this thesis I will make a distinction between the terms mate preference and mate choice.

\section{The evolution of mate choice}

\section{Direct selection}

Mate preferences can evolve under direct selection if individuals can gain direct benefits from being choosy (Kirkpatrick and Ryan 1991; Kokko et al. 2003). Through direct benefits choosers can reduce their investment in the current breeding event, which increases their prospects for future reproductive success or survival. Direct benefits associated to the choice for a high-quality partner can be nuptial gifts (Vahed 1998), fertilization insurance, access to resources or territory quality (Alatalo et al. 1986), and parental care (Hadfield et al. 2006; Nakagawa et al. 2007; García-navas et al. 2012). For instance, in blue tits (Cyanistus caeruleus), it has been shown that the breeding success is mainly determined by parental, rather than genetic effects (Hadfield et al. 2006) and that parental breast plumage colouration is positively correlated with provisioning rates and breeding success (Senar et al. 2002; García-navas et al. 2012). Thus, female blue tits may be able to increase their reproductive success by choosing for males with very yellow breast plumage who provision their offspring more than pale males.

\section{Indirect selection}

When choosers can secure genetic benefits via their offspring by mating with certain individuals there is an indirect selection for preferences for these individuals. Indirect benefits are additive, when an individuals' genetic traits are passed on to the offspring, giving them a higher relative fitness by increasing their survival and/or reproductive success (Trivers 1972; Iwasa et al. 1991; Kokko et al. 2006). This idea assumes that there is additive genetic variation in fitness, and that choosers can assess this heritable genetic quality in courters when choosing a mate. These additive genetic benefits can be divided into two categories: 'good-genes' and 'sexy sons'.

In the case of the good-genes hypothesis, choosers are thought to prefer certain traits in a mate that indicate their heritable viability (Møller and Alatalo 1999). Only high-quality courters can afford to maintain or develop these traits, ensuring the honesty of the signals (e.g. by giving a handicap to the carrier (Zahavi 1975), or by indicating resistance to parasites (Hamilton and Zuk 1982). Since viability or quality is dependent on a large number of alleles, good-gene indicators are expected to reflect genome-wide quality (Andersson and Iwasa 1996). However, it is difficult to show this good-genes effect empirically. Although Møller and Alatalo (1999) found a weak, but significant effect of 
male attractiveness on offspring viability, a later meta-analyses on a larger set of studies failed to show such a correlation (Prokop et al. 2012). In many studies no distinction is made between additive genetic effects and variation in maternal effects or epigenetic effects, which makes it difficult to demonstrate these good-genes effects (Hettyey et al. 2010).

In the case of the sexy-sons hypothesis, the focus is more on attractive offspring, rather than viable offspring. In this case the trait of interest does not have to signal genome-wide quality, since the indirect benefits are realised through higher mating success rather than through a higher viability (Andersson and Iwasa 1996). Therefore, if there is a populationwide preference for a certain trait, mutations increasing the expression of this trait will directly be selected for, regardless of any potential negative effects on survival or viability. Under this hypothesis, if choosers that are choosy mate more often with courters with a high expression of the trait, alleles for the preference for the trait and alleles for the expression of the trait will become linked (via linkage disequilibrium), which can result in a significant positive covariance between the preference and the display (Kokko et al. 2006). This can lead to runaway selection where the preferences for and the expression of the trait co-evolve and are driven to extremes (Fisher 1930; Lande 1981; Kokko et al. 2002).

While good-genes and sexy-sons were previously often seen as two mutually exclusive processes, recently it has been suggested more and more that these two hypotheses may not be as dissimilar as is often suggested. Both sexy-son and good-genes benefits can result from the same process of female choice for males with high breeding values for fitness (Eshel et al. 2000; Kokko et al. 2002). Which type of benefits determine preferences depends on the costliness of choice. If choice is cheap, leading to a high mating skew among males, the breeding value for the fitness of males is mainly determined by the sexiness of his sons. However if the costs of choice are high, the breeding value for fitness also has to include other components of fitness and therefore choice for viability - good genes, will play a larger role (Kokko et al. 2002).

\section{Exploitation of pre-existing biases}

Preferences for certain traits may have been selected for in other contexts than a mating context, and may be the result of a naturally selected sensory bias or differences in environmental conditions (Rodd et al. 2002; Kraaijeveld et al. 2011). For instance in guppies (Poecilia reticulata), who have a sensory bias for orange food items to optimize their foraging, it is thought that the intensity of male orange colouration has been selected for though the choosers' bias for this colour in their choice of a mate (Rodd et al. 2002). 


\section{The 'Lek paradox'}

Most of the theory described above suggests that all individuals should have the same preferences for the same universally best individuals, or superior traits. However, if all choosers consistently choose for the same courter traits indicating quality, as a consequence this will rapidly exhaust the genetic and phenotypic variation and will drive the traits to fixation. When this happens, the genetic benefits of preferring and choosing such traits will decrease. This apparent paradox is called the 'Lek paradox' (Borgia 1979; Kirkpatrick and Ryan 1991; Rowe and Houle 1996; Kotiaho et al. 2008). Nevertheless, empirical evidence shows that substantial variation still persists in courters' traits, despite this often-assumed directional selection. Solving this paradox is a long running and much debated issue in the study of sexual selection (Kirkpatrick and Ryan 1991; Kotiaho et al. 2008). Numerous attempts to solve the problem have been done by considering condition dependence of male ornaments (the handicap principle; Iwasa et al. 1991), selection for non-additive traits, and fluctuating selection, but no consensus has been reached yet. Currently, there is a growing acceptance that individual variation in mate preferences may weaken the strong directional selection on male ornaments and thereby allow genetic diversity to persist (Jennions and Petrie 1997; Widemo and Sæther 1999; Brooks and Endler 2001; Forstmeier and Birkhead 2004; Mays and Hill 2004).

\section{Individual differences in mate preferences and compatibility}

Previously, it was assumed that all individuals should have the same preferences for the same superior individuals. Empirical research, however, has shown that this is not always the case and that choosers can actually differ consistently in their preferences (Godin and Dugatkin 1995; Brooks and Endler 2001; Forstmeier and Birkhead 2004; Lehtonen and Lindström 2008). Similarly courter attractiveness may not always be repeatable between choosers, suggesting variation in chooser preferences (Forstmeier and Birkhead 2004). This variation in preferences has been suggested to be due to differences in physical condition, in ontogeny, and in the environment (Jennions and Petrie 1997; Widemo and Sæther 1999; Cotton, Small, et al. 2006): when the costs of being choosy are too high for the individual to bear, for instance due to increased sampling costs or a lower condition, individuals will be less selective in finding a mate according to their preferences (Milinski and Bakker 1992; Cotton, Small, et al. 2006).

Sometimes, however, the same courter may give higher benefits to some choosers than to others. Interfering with free mate choice tends to harm choosers and lowers the average reproductive success (Gowaty et al. 2007). In mice (Peromyscus polionotus rhoadsi; Koeninger Ryan and Altmann 2001; Mus musculus; Drickamer et al. 2003; Gowaty et al. 2003), zebra finches (Taeniopygia guttata; Ihle et al. 2015), fruit flies (Drosophila 
pseudoobscura; Anderson et al. 2007) and giant pandas (Ailuropoda melanoleuca; MartinWintle et al. 2015) it has been shown that reproductive success and offspring viability were higher when individuals were paired with preferred rather than unpreferred partners, irrespective of the partner's phenotype. In these cases, compatibility rather than quality seems to be important for reproductive success. Thus, when an individual is mated with a compatible partner, it has a higher reproductive success than when mated with a different (incompatible) partner, regardless of each pair mates' separate qualities.

Socially monogamous species are especially likely to have evolved individual preferences for compatible mates rather than uniform preferences, due to the fact that individuals become unavailable after pairing. In these monogamous systems, strong preferences for attractive individuals may not be favoured by selection, since scramble competition over attractive mates leads to increased costs of choosiness, which may not outweigh the benefits (Dechaume-Moncharmont et al. 2016; Wang et al. 2017). Instead of uniform preferences it may therefore be more beneficial to have individual preferences for compatible mates, which leads to optimal direct or indirect benefits (Ihle et al. 2015; chapter 3 ). Thus, there may be adaptive explanations for variation in mate preferences. In the literature, individual variation in preferences and preferences for compatibility may have been somewhat overlooked, since selection for compatibility may reduce variation in male mating success and consequently is likely to reduce the strength of populationwide sexual selection (unless the preferences are very strongly assortative that it will lead to speciation). Benefits of preferences for compatibility, similar to universal directional preferences, can be direct or indirect.

\section{Indirect benefits of compatibility}

In the case of non-additive genetic variation in fitness-relevant traits it is not directly the individual quality of the parental genomes, but the combination of these genomes that affects offspring fitness (Zeh and Zeh 1996; Neff and Pitcher 2005; Puurtinen et al. 2009). Most of the models on genetic complementarity are based on the assumption that offspring fitness is related to their heterozygosity (Tregenza and Wedell 2000; Neff and Pitcher 2005; Kempenaers 2007; Mays et al. 2008). Heterozygosity, the genetic variability within an individual, is known to be positively correlated with reproductive success, survival, immunocompetence and parasite resistance (Kempenaers 2007; Chapman et al. 2009). The most common example of genetic (in)compatibility is perhaps inbreeding and the homozygote disadvantage in offspring from related individuals (inbreeding depression). This disadvantage is thought to be due to an accumulation of recessive deleterious alleles in homozygous genotypes causing a fitness disadvantage, or due to the advantage that heterozygote individuals have. This heterozygote advantage can either be the result from masking deleterious alleles when paired with a dominant allele, or by 
overdominance or heterosis, giving heterozygote individuals a fitness advantage (Charlesworth and Charlesworth 1987; Mays et al. 2008).

By finding an unrelated, or genetically dissimilar mate, offspring heterozygosity can be increased and the negative effects of inbreeding avoided when inbreeding depression is larger than any negative effects caused by extreme outbreeding (i.e. outbreeding depression; Van de Casteele et al. 2003; Szulkin et al. 2007). While there is ample evidence of inbreeding depression (Keller and Waller 2002; Spottiswoode and Møller 2004), the evidence of inbreeding avoidance is not as clear (reviewed in Kempenaers 2007). Some studies found disassortative mating patterns for genetic similarity (Freeman-Gallant et al. 2003; Marshall et al. 2003; Gerlach and Lysiak 2006; Oh and Badyaev 2006; Sanderson et al. 2015), other studies have found mating patterns do not differ from random (EdlyWright et al. 2007; Hansson et al. 2007; Jamieson et al. 2009), or have even found choice for genetically similar mates (Cohen and Dearborn 2004; Loyau et al. 2012; Robinson et al. 2012). Even within species mating patterns with regard to genetic similarity have been found to vary, such as variation in mating patterns between populations in blue tits (Krokene and Lifjeld 2000; Foerster et al. 2006). It should be noted however, that most of these studies measure mate choice, the partner an individual eventually pairs with, and not mate preference.

There is an on-going discussion about the possible reasons underlying inbreeding tolerance. Inbreeding avoidance may not be necessary in species with sufficient natal dispersal (Szulkin et al. 2012), or the risk of inbreeding depression may not weigh up to the costs associated with inbreeding avoidance. In addition, these costs may be dependent on the level of inbreeding in the population (Kempenaers 2007; Jamieson et al. 2009), which may lead to between population differences in inbreeding avoidance. Additionally, in species with biparental care, genetic compatibility may be traded off against direct benefits or compatibility, such as territory quality, or paternal care (Mays et al. 2008). In this case choosers have been shown to have more extra-pair matings when paired with genetically similar social partners (Freeman-Gallant et al. 2006; Brouwer et al. 2011; van Oers et al. 2008; reviewed in Arct et al. 2015, but see also Foerster et al. 2006; Edly-Wright et al. 2007; Rubenstein 2007), potentially enabling them to increase their offspring heterozygosity. Alternatively, inbreeding avoidance may not always be the best strategy. In some cases there may be an optimal level of inbreeding/outbreeding due to outbreeding depression, or benefits associated with kin selection or inclusive fitness benefits may lead to random mating or even mating patterns for genetic similarity (Tregenza and Wedell 2000; Mays and Hill 2004; Kokko and Ots 2006). 


\section{Direct benefits of compatibility}

Direct benefits of compatibility may also play a role in mate preferences, emerging from a combination of both parents' behaviours. Especially in species with pair bonds and biparental care, behavioural compatibility is expected to play a role in reproductive success (Schuett et al. 2010). Likely candidates for behavioural compatibility are expected in individual differences in personality traits, such as boldness, exploratory behaviour or aggressiveness (see Royle et al. 2010). The combination of personalities within a pair has been shown to affect reproductive success (Dingemanse et al. 2004; Both et al. 2005), and it is likely that these benefits are mediated through parental care (Royle et al. 2010; Schuett et al. 2011). In a number of species, mate preferences or choice for behavioural similarity have been reported (Schuett 2008; Kralj-Fišer et al. 2013; Class et al. 2014). Behavioural compatibility can increase the likelihood of reproducing, pair coordination and success (Schuett et al. 2011; Ariyomo and Watt 2013; Jiang et al. 2013; Kralj-Fišer et al. 2013; Class et al. 2014). For instance in cockatiels (Nymphicus hollandicus) pairs with more pair bonding behaviour had a higher incubation coordination and subsequently hatched more eggs (Spoon et al. 2006), and in zebra finches behaviourally similar pairs were more successful in rearing their offspring (Schuett et al. 2011).

It is likely that depending on their behavioural compatibility pairs may differ in their coordination of parental care (Johnstone et al. 2014). Personality types may vary for instance in their provisioning rules, risk taking, behavioural consistency or the responsiveness to their partners' efforts (Both et al. 2005; Hinde 2006; Hinde and Kilner 2007; Royle et al. 2010; Schuett et al. 2011; Gabriel and Black 2012; Mutzel et al. 2013; David et al. 2015). Personality-based differences in perception and response to partner foraging effort, consistency and strategy may in turn influence a parent's own investment. When individuals have similar personalities, and thus similar provisioning rules or strategies, this may lead to greater coordination of care, greater investment, and subsequently a higher reproductive success (Royle et al. 2010; Schuett et al. 2011).

\section{Self-referent preferences}

Different courter traits may indicate different benefits to choosers (Johnstone 1995; Johnstone 1996; Candolin 2003). Depending on the choosers traits some benefits may be more important or more beneficial to some than to others. However, if and how courters differ in the attention they pay to different mate qualities and how they weigh different signals of quality has received relatively little attention. Self-referent preference may also be related to the choosers own traits such as size or quality. For instance in convict cichlids (Amatitlania nigrofasciata) females preferred a male that was approximately $30 \%$ larger than themselves, and thus large females preferred relatively large males, and smaller females showed a preference for relatively small males (Dechaume-Moncharmont et al. 2013). 
Individual quality may not only affect choosiness (as mentioned earlier), but it is also suggested that there has the potential to affect preferences. Low-quality individuals with a preference for high-quality mates may try in vain to obtain such a high-quality mate even though their competitive ability is not high enough to get one, or to keep one. To avoid these costs of competition it is suggested that individuals with poor competitive ability may have evolved preferences for mates with a similar quality to themselves (Fawcett and Johnstone 2003; Holveck and Riebel 2010).

Additionally, choosers may differ in their preference, depending on whether individuals choose for direct or indirect benefits. Females may increase their reproductive success by choosing males with high parental care or a good territory when choosing a social mate (Senar et al. 2002), but may focus on genetic quality or compatibility when choosing an extra-pair mate (Foerster et al. 2003; van Oers et al. 2008). But choosers may also differ in how they weigh courter traits, depending on their own traits and qualities, such as their ability to compensate for reductions in parental care (Burley 1986; Sheldon 2000) or for certain genetic characteristics (Pilakouta and Smiseth 2017). For instance, in burying beetles, inbred females had strong preferences for outbred males, while outbred females did not show such preferences. It seems that the inbreeding status of the male was very important for inbred females to increase their offsprings' genetic variability, while for outbred females this trait was much less important for their choice of a mate, since they themselves were outbred enough to ensure their offsprings' genetic variability (Pilakouta and Smiseth 2017). Choosers may also pay more attention to genetic quality than to direct benefits in a mate, when they themselves are able to compensate for the potentially reduced parental care and vice versa. Thus, having these self-referent preferences, can optimise choice for each individual depending on their own qualities and weaknesses.

\section{Plastic preferences}

Preferences may also differ depending on the physical or social environment and to depend on the state of the chooser. For instance, environmental circumstances can affect preferences depending on the expected benefits of certain courter traits in that environment. Consequently, chooser selection for traits in a mate may also vary between years. In lark buntings (Calamospiza melanocorys) female selection for male traits was found to differ between years, with even reversals of direction of selection for some traits (Chaine and Lyon 2008). Females were shown to be very flexible in their choice for mates, with large between-year differences in choice for male traits, and the changes in these preferences paralleled the changes in expected benefits from male traits for that year. In collared flycatchers (Fidecula albicollis), there were differences in the strength of preference even within seasons: only late breeding females showed strong preferences for the male forehead patch size, a sexually selected trait, which coincided with the reproductive 
benefits they can gain from this. In late breeding pairs male patch size correlated with reproductive success, while this benefit it not present in early breeding females (Qvarnström et al. 2000). This suggests that differences in female preferences within the same population may result from short-term environmentally driven changes in the expected benefits from the choice for males with certain traits. Similarly, it is suggested that the social environment an individual experiences may affect its preferences (Rodríguez et al. 2013). For instance, mate preferences of female wolf spiders (Schizocosa uetzi) for male leg colours, were determined by their experience with black- or brownlegged males when they were immature (Hebets 2003). If they were familiar with blacklegged males they preferred males with black legs, and vice versa, whereas when they had no experience with either of them, they showed no preferences. Thus, the environment, social or physical, and the expected benefits from certain traits in a mate can influence chooser preferences in order to optimize reproductive success in a given environment.

\section{Mate choice}

Many variables play a role in the translation of preference of the chooser into choice, both internal (e.g. sampling strategy and choosiness) and external (e.g. competition over mates and local availability). Due to these variables, choice is often not the same as preference, and these two should therefore be considered as separate processes, both playing an important role in selection. For instance, choice patterns for size in orb weaving spiders (Zygiella $x$-notata) may suggest assortative preferences for size. But when individuals are given a choice in the absence of (or under low) intrasexual competition, all males will prefer the largest and most fecund females and the size assortative mating pattern disappears (Bel-Venner et al. 2008). Similarly, in numerous studies, assortative mating patterns are thought to be the result of competition for mates leading to assortative mating for a certain quality trait, without considering the possibility of assortative preferences for compatibility instead of quality (García-navas et al. 2009; Holveck and Riebel 2010). While often the terms mate preference and mate choice are used interchangeably, here I strongly advise that the terminology for mate preference (functions) and mate choice should be used consistently, to be able to compare studies and draw conclusions (Jennions and Petrie 1997; Wagner 1998; Edward 2015). Only by considering both preference and choice, with both playing a role in sexual selection, is it possible to disentangle these separate effects and to clarify how selection acts on preferences and traits.

\section{Differential allocation}

As described earlier, individuals can gain direct or indirect benefits from mating with a compatible partner, leading to increasing reproductive success. But these benefits are not the only effects that mate attractiveness can have on reproductive success. Parents trade- 
off their current and future reproduction by allocating resources to reproduction over time (Houston and McNamara 1999), and have been selected to do so in response to the expected costs and benefits of these current and future reproductive opportunities (Brommer 2000). The attractiveness of a current mate affects the trade-off between current and future reproductive opportunities, and thus the optimal investment (Ratikainen and Kokko 2009). This idea of differential allocation depending on mate attractiveness was first presented by Burley $(1986,1988)$ to explain differences in reproductive success after artificially increasing mate attractiveness. Differential allocation was originally assumed to entail a higher reproductive success with increasing mate attractiveness or quality (reviewed in Sheldon 2000 and Horváthová et al. 2012). However, several studies have shown a decrease in reproductive investment with increasing mate quality (Bluhm and Gowaty 2004; Byers and Waits 2006; Gowaty et al. 2007; Bolund et al. 2009), initially termed reproductive compensation (Gowaty et al. 2007). Currently, however, differential allocation and reproductive compensation are seen as the ends of a continuum of reproductive investment patterns depending on mate qualities (Harris and Uller 2009; Ratikainen and Kokko 2009). Modelling approaches have attempted to determine when and how investment should change depending on mate quality and attractiveness (Harris and Uller 2009; Kindsvater and Alonzo 2014; Haaland et al. 2017), and this seems to depend on how mate quality benefits reproductive success, and the choosers age or energetic state, and prospects for future reproduction and future distribution of mate quality (Harris and Uller 2009; Kindsvater and Alonzo 2014).

\section{Aspects of mate preference and choice}

Here I distinguish between three different aspects of mate preferences that may depend on the choosing individual (Figure 1.1): preference functions (What to choose?), sampling tactic ('How to choose?') and choosiness ('How much to invest?'). 


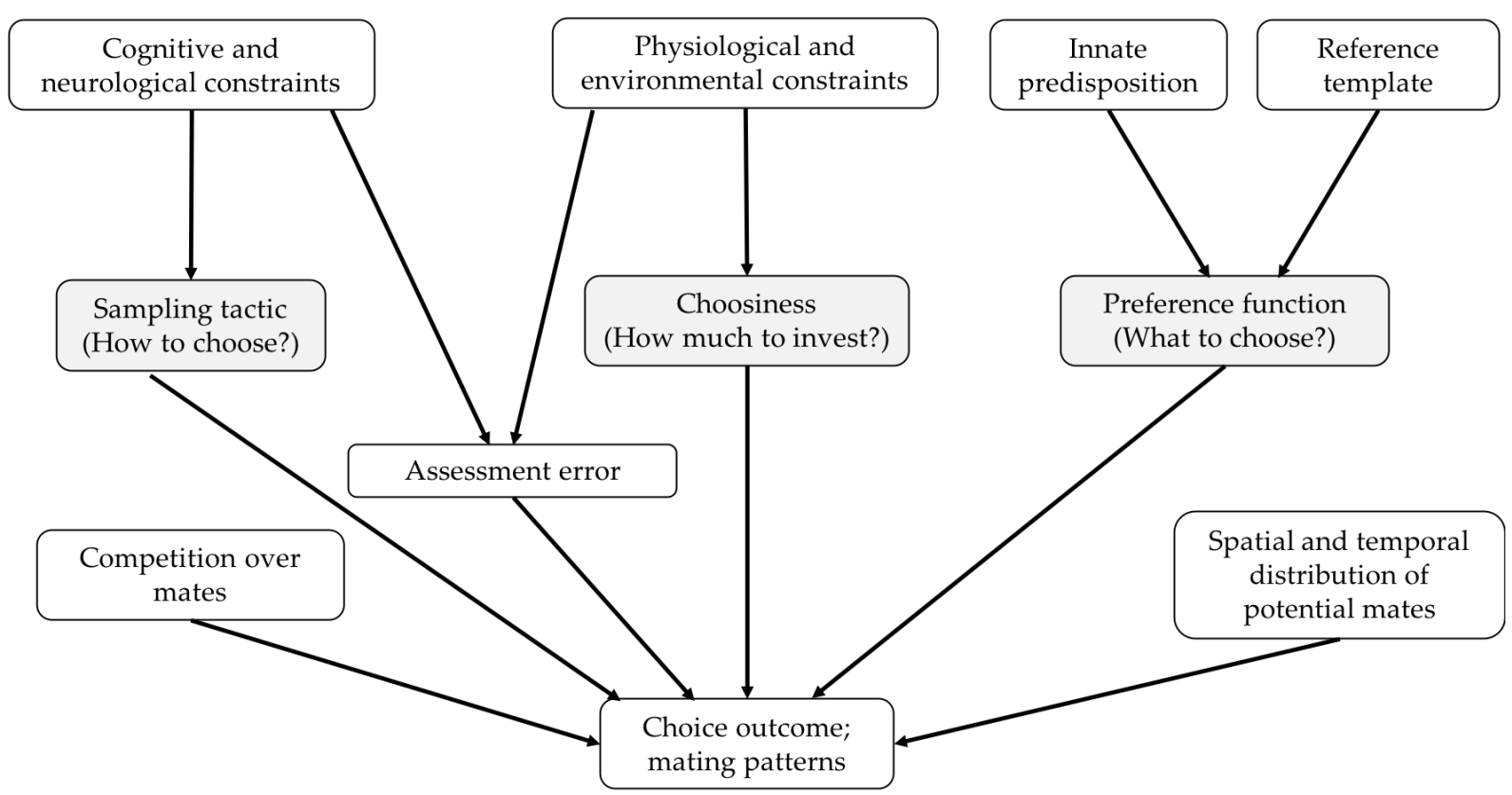

Figure 1.1 - From preference to choice: the figure illustrates important aspects that play a role in mate preferences and mate choice.

\section{Preference functions: What to choose?}

Preferences or the ranking of stimuli (Jennions and Petrie 1997; Widemo and Sæther 1999) can be characterised as preference functions (Wagner 1998), in which the mating response varies with the value of the trait. Perhaps the simplest form is the preference threshold, in which all trait values above a certain threshold are preferred equally, while all values below this threshold are rejected (Figure 1.2a). Preference functions can also be directional, when preferring larger or smaller values or can be unimodal, in which there is a more intermediate optimum to their preference (Figure 1.2b: unimodal preference; Wagner 1998; Edward 2015). But more complicated preferences are found such as optimal or disruptive preferences can occur (Figure 1.2c-d: bimodal preference; (Sappington and Taylor 1990; Turner et al. 1995; Rosenthal 2017). When preferences are largely driven by single traits, preferences will generally be directional or unimodal. However, when preferences are complex, integrating multiple traits or multiple cues, this may result in more complex preference functions such as disruptive (multimodal) preferences. Eventually, such disruptive preferences may lead to speciation (Turner et al. 1995). 

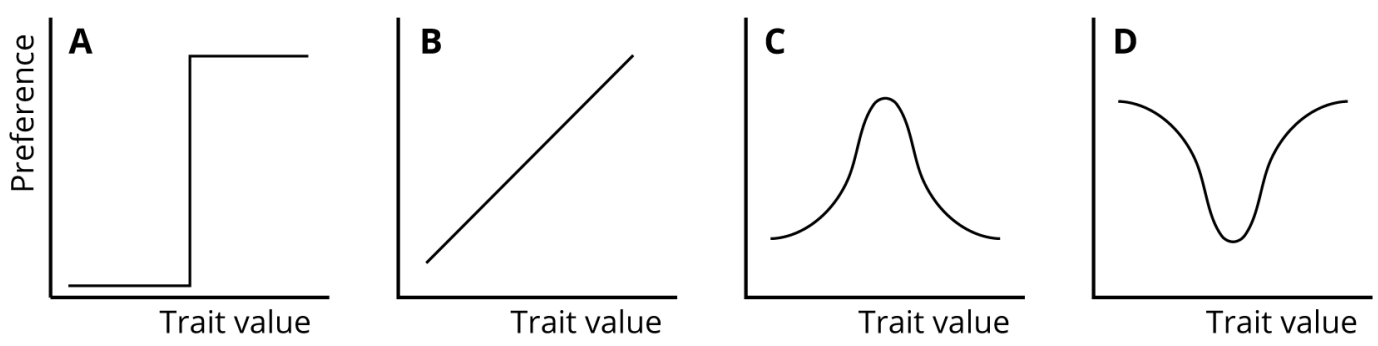

Figure 1.2 - Variation in preference functions. Each graph depicts variation in trait values of potential mates ( $x$-axis) and the preference or investment of resources into these potential mates (y-axis). A) preference threshold; $\boldsymbol{B}$ ) directional preference; $\boldsymbol{C})$ optimal preference; $\boldsymbol{D}$ ) disruptive preference.

\section{Sampling strategy: How to choose?}

To translate internal preferences into choice, individuals have to sample potential mates. How they sample the available options and by which strategy they evaluate them can greatly affect their choice of a mate (Janetos 1980). Typically, sampling is divided into two extremes of mate-encounter scenarios. On the one hand, individuals encounter courters sequentially - one at a time, and on the other hand, individuals may encounter multiple courters simultaneously. How individuals evaluate these courters (e.g. by evaluating the available options comparatively or by assigning an absolute attractiveness value to certain trait values) and how individuals subsequently choose their partner (e.g. by choosing the best option from a certain number of sampled courters, 'best-of-n' or by choosing the first courter that exceeds a certain threshold, 'fixed threshold rule') depends on their sampling strategy (Janetos 1980; Bateson and Healy 2005; Dechaume-Moncharmont et al. 2016). Chapter 2 addresses how individuals compare potential partners.

\section{Choosiness: How much to invest?}

Choosiness can be described as the time or effort that a chooser is prepared to invest in a choice (Brooks and Endler 2001). Choosiness can be divided into two components: 1) responsiveness and 2) discrimination. Responsiveness is the mean response by a chooser over the distribution of available options and corresponds to its motivation to mate. Therefore this attribute of its sampling behaviour is likely to be correlated with its physiological state and the environment (Brooks and Endler 2001). On the other hand, discrimination reflects the choosers' variance in response among the sampled options. When discrimination is low all options receive a similar response, while when discrimination is high there is a large bias in response towards one (or a few) potential mates (Toomey and McGraw 2012). This aspect of choosiness may also be condition or environment dependent. For instance in crickets, females with a high body condition showed a higher responsiveness and more discrimination in their responses to male call 
characteristics than females with a low body condition (Holman and Kokko 2014). Similarly, female guppies decreased both their responsiveness and discrimination in their preferences for brightly coloured males when a predation risk was present (Godin and Briggs 1996).

\section{Availability of potential mates and environmental constraints}

A chooser has to be selective about which of the potential partners that it has encountered it will mate with (Byers et al. 2005). When the density of potential partners is low, or searching costs are high, the number of mates sampled by the chooser will decrease. The smaller this sample, the weaker the correlation between the preferences and the benefits of having these preferences will be (Benton and Evans 1998). This weakening of sexual selection occurs because if choosers are limited to a small sample of suboptimal potential mates, they will still choose one of them to mate with, despite it not being preferred. Thus, the availability of potential mates can have large effects on the choices made, and with it, on the strength of selection.

\section{Competition over mates}

Since gametes are a limiting 'resource', courters compete over the access to these gametes amongst each other. Therefore, high competition over mates may limit the access to potential mates, through for instance interfering competition or scramble competition (Danchin and Cézilly 2008; Dechaume-Moncharmont et al. 2016). Under high competition or intra-sexual competition mate searching costs often increase which may lead to a lower choosiness and can influence mating patterns (Jennions and Petrie 1997; Wagner 1998; BroJørgensen 2002; Dechaume-Moncharmont et al. 2016).

\section{Choice outcome}

The eventual choice of a mate results from the combination of preferences, sampling strategy, choosiness and the environmental constraints (competition and local availability of potential mates). Since so many variables play a role in translating preference into choice it is important to realise that the mate that an individual obtains (mate choice) may not represent its mate preference, as is often assumed. Therefore preference and choice should be considered as two distinct processes, both of which should be studied independently to fully understand how selection may act upon preferences (Jennions and Petrie 1997; Wagner 1998; Widemo and Sæther 1999; Edward 2015). In chapters 3 and 4 I compare preference with choice and study the effects on reproductive success.

\section{Measuring mate preferences}

The outcome of mate choice and thus the impact and action of sexual selection on courter traits can readily be observed in a population. However, to better understand how 
selection works we need to learn more about how preferences, sampling and the effects of the external factors interact to result in this eventual partner choice (Wagner 1998; Widemo and Sæther 1999). These can only be done experimentally. Similarly, when testing mate preferences it is crucial to consider a species' sampling tactic (or evaluation method) and what is ecologically relevant for this species (Bateson and Healy 2005; Castellano et al. 2012; Dougherty and Shuker 2014).

An important way in which experimental designs measuring preferences differ lies in the number of options or stimuli the chooser is presented with: the choice design (Dougherty and Shuker 2014). Tests can use a no-choice or a choice design (Wagner 1998). In a nochoice design the chooser is presented with one stimulus at a time, often showing different stimuli sequentially. In contrast, in a choice design, choosers are presented with more than one stimulus individual simultaneously allowing for comparative evaluation of potential partners (Edward and Chapman 2011). Typically, 2 stimuli are presented to measure a directional preference. However, when preferences are actually disruptive or stabilising, two-choice tests may lead to confounded conclusions, especially when choosers are only presented with extreme courter trait values. By presenting choosers with 4 or 6 stimuli it is also possible to measure disruptive or stabilising preference functions. Often these tests are repeated with different groups of stimuli to obtain more reliable preference functions (Wagner 1998).

Depending on the study species and how it evaluates and encounters potential partners, there are advantages and disadvantages for both no-choice and choice design. The sampling strategy and evaluation method that a species uses can strongly influence the strength of the results and the conclusions drawn (Castellano et al. 2012). When comparison is possible in 2-choice tests, it is possible to detect relative, directional preferences between stimuli (Wagner 1998; Maclaren and Rowland 2016). Therefore, these tests may give stronger results, since even small differences in stimulus trait values may lead to large differences in chooser responses. However, this may also lead to an overestimation of the effects, especially when dichotomous yes or no choice outcomes are recorded (Wagner 1998). In the case of no-choice tests however, choosers don't have the possibility to compare options, possibly making preference patterns weaker, and it gives the possibility for order effects, when the previously presented stimuli affect responses to later shown stimuli. Additionally, if choosers perceive no-choice tests as an environment in which there is an exceptionally low mate encounter rate, the perceived costs of rejecting a potential mate are also much higher, leading to confounded measures of preference (Barry and Kokko 2010; Booksmythe et al. 2011). Thus, consideration of the species, its evaluation of potential mates and its ecology are crucial for the choice of the most appropriate test design (Castellano et al. 2012; Dougherty and Shuker 2014). Chapter 2 
focuses on how great tits compare potential partners and how this may affect results depending on the choice design used.

\section{The aim of this thesis}

While in many studies population-wide preferences are assumed, more and more studies suggest that individuals differ in their preferences, and may actually prefer compatible mates rather than universally attractive individuals. These differences in preference can be adaptive and may depend on the choosers' own traits. Moreover, several studies on captive populations have found that the fitness benefits from mating with a certain individual may differ between individuals, suggesting individual differences in preference and both direct and indirect benefits of mating with these compatible mates. However, it is still unclear how perceived mate attractiveness and compatibility affect reproductive success in a wild population.

Additionally, while there is a vast base of literature regarding mate preferences, mate choice and the fitness effects of choice, in these studies, observed mating patterns are often assumed to represent preference, even though they result from the interaction between preference, sampling strategy, choosiness and environmental factors. Therefore, the mate that an individual obtains (mate choice) may not represent its mate preference, as often assumed. Instead, to understand fitness consequences and the evolution of mate preferences, preference and choice should be studied as two distinct processes. To our knowledge, no study thus far has combined all three and tested what the fitness benefits of mate preferences are under mate choice constraints in a wild population.

Therefore, the overall aim of this thesis was to study the role of individual differences in mate preferences and its effects on reproductive success.

- Do individuals differ in their preferences for a mate?

- How are mate preferences reflected in mate choice?

- How does pair (in)compatibility affect reproductive success? 


\section{Study system}

\section{Species}

In this study we used wild great tits (Parus major) to test mate preferences and mate choice. This non-migratory passerine bird occurs widespread throughout Europe, the Middle East and Asia. Great tits live in deciduous or mixed forests but can also commonly be found in gardens. In the summer they are predominantly insectivorous, while in the winter they mainly live on plant-based materials such as nuts and seeds (Gosler 1993).

Great tits are a key model species to study behaviour in the wild and have been widely studied for decades to link variation in behaviour to fitness. Because great tits (in this part of Europe) rarely migrate they can be monitored throughout the year and, being a cavitybreeder, their reproductive success can be measured accurately using data collected from pairs breeding in nest boxes. Between spring and autumn, great tits live in pairs and occupy a territory in the forest. In the Netherlands it has been shown that already in December the total breeding density for the following breeding season is determined (Kluijver 1971). The birds roosting in nest boxes during the winter months are the territory owners who will also breed in this territory in the subsequent breeding season. During the winter they often forage in larger fission-fusion foraging flocks, whilst maintaining local dominance in their own territories (Hinde 1952; Gosler 1993). This period between late autumn and early spring is also the time during which great tits pair up (Drent 1983; Dhondt et al. 1996; Culina 2014). Not only first year birds will pair up for the first time during the winter period, but also older birds, after divorce or the death of a partner will choose a new mate (Dhondt et al. 1996). In a study on great tits in Wytham Woods (UK), it was shown that pairs that ended up breeding together already started to associate increasingly during the winter months (Culina 2014), and that this happens throughout the entire winter period. After removal of their mate, just before egg laying started, males had difficulty finding a new partner to breed with, which suggests that during the breeding season only few unmated individuals remain (Slagsvold et al. 1994). Consequently, if most individuals are mated during the breeding season, the last individuals to mate will automatically have very limited choice between potential mates, which may cause high variation in compatibility between pairs.

Within their territory the pair chooses a natural cavity, or - in our case - a nest box to breed in (Kluijver 1971). After the female has built the nest from moss and animal hairs, she will lay between 3-13 eggs (Tinbergen and Daan 1990; Gosler 1993). The eggs are incubated by the female for 13-15 days, after which parents feed the offspring in the nest box for 18-21 days until fledging. During this period both males and females invest heavily in their 
offspring: both parents provide parental care and provision their offspring with insects such as caterpillars, spiders, moths, and flies (Gosler 1993).

\section{General study methods}

\section{Great tits as study species for mate preferences}

Here we use great tits as a model species to measure differences in mate preferences, mate choice and reproductive success in a wild population. This species is particularly suitable since it breeds in a nest box population and therefore can be monitored easily throughout the year, is known to be relatively stress resilient and will participate in experiments in the lab. Additionally, since this species is socially monogamous, we can observe which partner they paired up with (mate choice) and observe their coordination of parental care. Using this unique approach, we can combine measurements of mate preference in the lab with observations of mate choice in the field, and directly measure the effects of parental compatibility under ecologically realistic conditions.

\section{Study populations}

I conducted my study in the study site Boslust in the Netherlands $\left(52^{\circ} 01^{\prime} \mathrm{N}, 5^{\circ} 85^{\prime} \mathrm{E}\right.$ : Figure 1.3 , area A). In this mixed deciduous and pine woodland 130 nest boxes have been distributed over an area of around 70 ha. Every year around 60 great tit pairs are found breeding in these nest boxes.

\section{Testing mate preferences}

Between January and March 2014 and 2015 we tested 70 males and 69 females for their preferences ('focal birds'). We caught these birds in the main study site Boslust while they were roosting in nest boxes. Great tits that roost in nest boxes are known to be territorial and keep local dominance in their breeding territories throughout the winter (Kluijver 1971). The preference tests took place in a using a carrousel shaped six-choice chamber (Figure 1.4). We presented each focal bird with 3 different groups consisting of 6 birds of the opposite sex ('stimulus birds'), who originated from different field sites than the focal birds (see Figure 1.3; areas B-F). With the six-choice test we were able to measure individual directional and quadratic (stabilising or disruptive) preferences (Edward 2015). Additionally, using this approach we made it possible for the birds to compare potential partners simultaneously (see chapter 3 ). During the winter great tits often forage in larger fission-fusion foraging flocks and it is therefore not unrealistic for this species to 


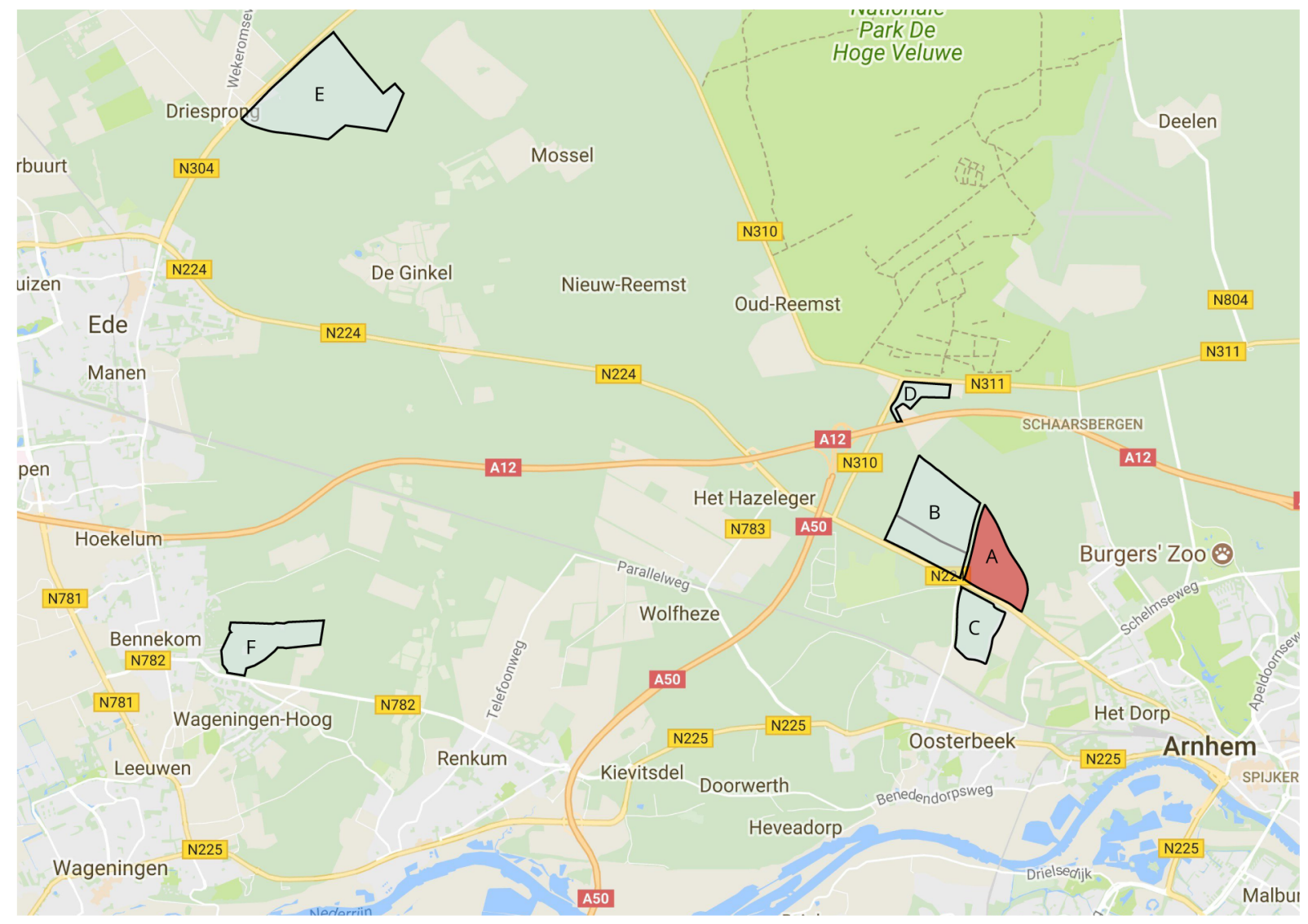

Figure 1.3 - Distribution of field sites from which we caught birds. Our main field site where we caught our focal birds and subsequently monitored all breeding attempts is A: Boslust. All stimulus birds originated from other field sites (B: Westerheide and Nuon; C: Lichtenbeek; D: Heijkamp; E: Roekelse bos; F: Bennekomse bos. (Adapted from Google maps, 2018).

simultaneously encounter six birds of the opposite sex. We recorded the time spent in front of each stimulus bird, which is commonly used as a measure of preference. Great tits do not show clear courtship or copulation solicitation behaviours outside the fertile period and it is therefore difficult to validate these tests and the association time measured using behavioural data. However, in numerous species association time in a choice test has been shown to predict courtship behaviours or pair formation (Clayton 1990; Hill 1990; Mays and Hill 2004; Witte 2006; Lehtonen and Lindström 2008; Dechaume-Moncharmont et al. 2011; Jeswiet and Godin 2011) and correlated with reproductive success (Drickamer et al., 2003). We therefore use this association time as a measure of preference. After 7 days in the laboratory all birds were returned to their field site of origin. By measuring trait values of both focal and stimulus individuals, we were able to analyse which traits in potential mates were important for preferences, and whether these preferences also depended on the choosers' traits. 


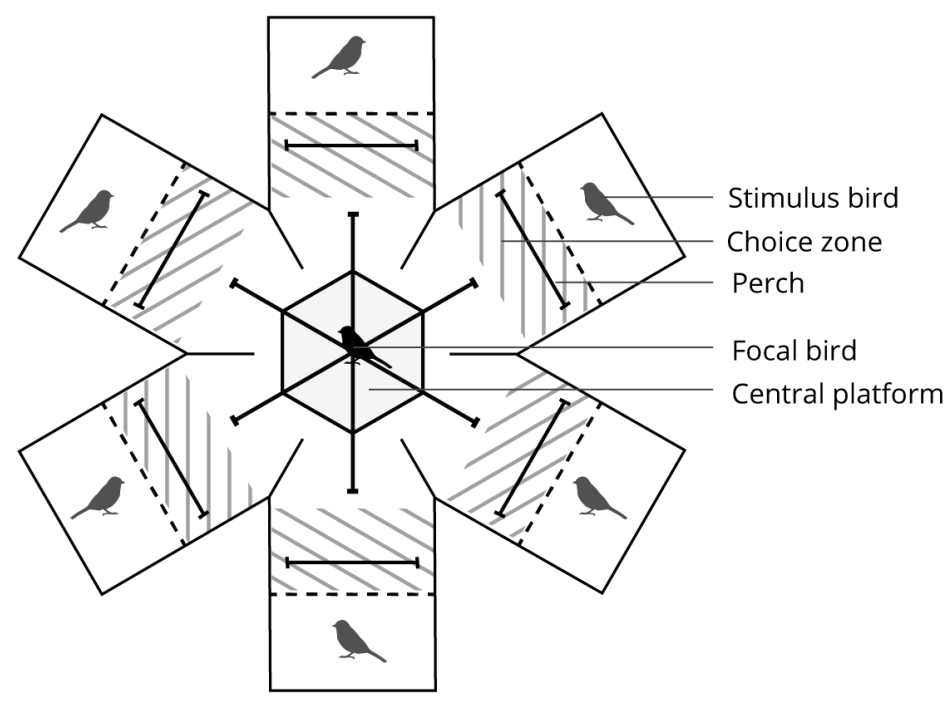

Figure 1.4 - Experimental setup mate preference test. Birds were tested for their preference in a sixchoice test room. Time spent in each of the choice zones was measured.

\section{Reproductive success}

Since great tits, after natal dispersal, rarely show breeding dispersal, we were able to follow the birds that we tested for preferences, and all other great tit pairs breeding in the Boslust field site, in the subsequent breeding seasons. During the breeding season, we checked unoccupied nest boxes at least twice a week for nesting activity. Once the nest was built and egg laying had started we estimated the egg-laying date, the start of incubation, hatching date and fledging date, and recorded the clutch size, brood size and the number of fledged offspring (Hinde 2006). By cross fostering offspring we were able to distinguish between direct and indirect effects of parental compatibility. Additionally, to study parental care in more detail, we recorded parental provisioning behaviour for each nest using RFID and video recordings. Using these recorders, we were able to record parental provisioning behaviour.

\section{Mating preferences in great tits}

Despite a number of correlative studies reporting mating patterns or fitness effects for several traits in great tits (eg. Norris 1990; Ko et al. 1999; Both et al. 2005; Quesada and Senar 2009; Sepil et al. 2015), so far no studies have actually tested preferences in great tits. We hypothesized that the following characteristics may play a role in mate preferences and reproductive success: plumage traits (yellowness and black breast stripe size), genetic characteristics (heterozygosity and relatedness), body condition and personality (see Figure 1.5). 


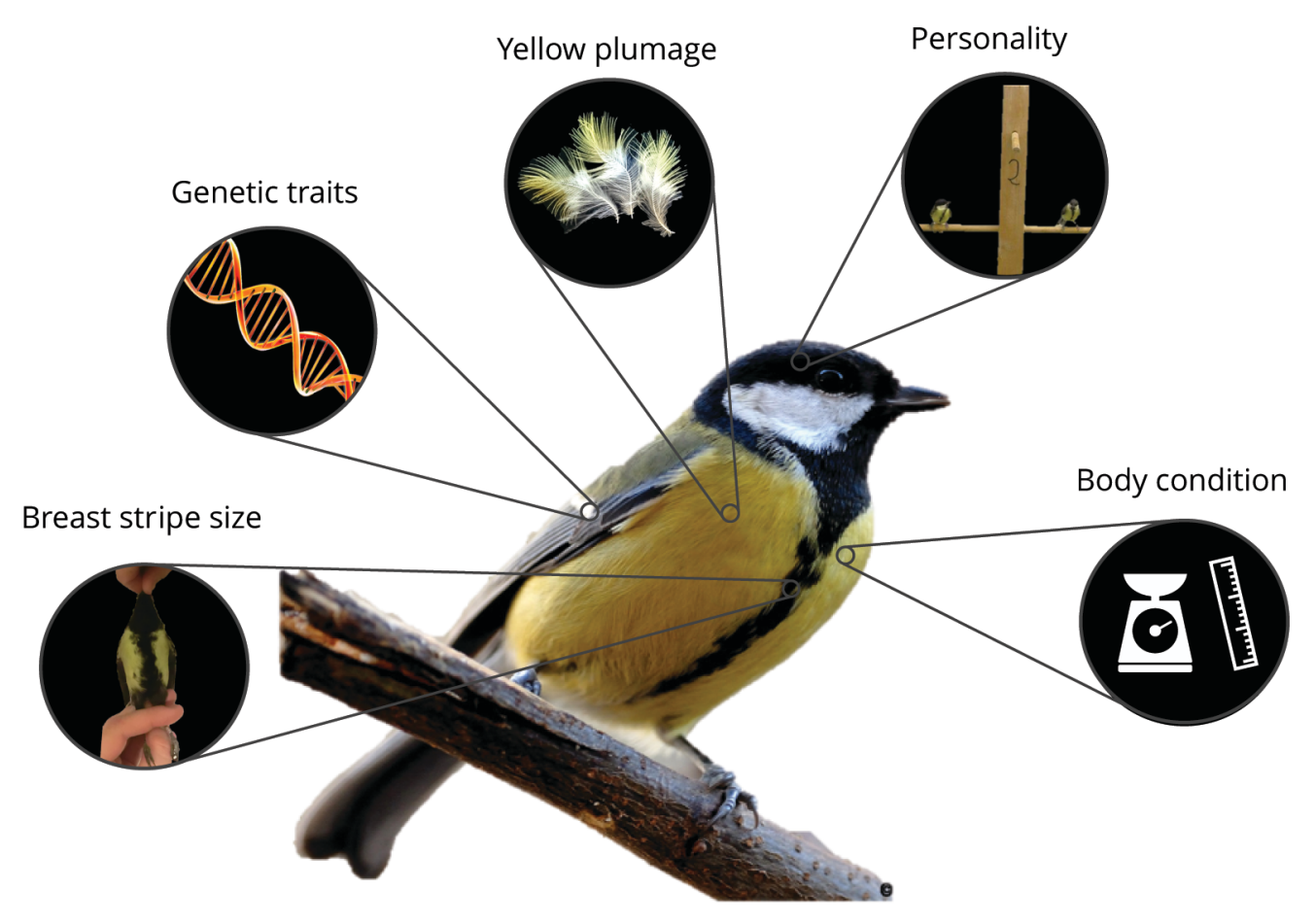

Figure 1.5 - Traits that were hypothesized to play a role in mate preferences in great tits: Breast stripe size; genetic traits such as heterozygosity and relatedness; yellow breast plumage coloration; personality; body condition.

\section{Plumage traits}

The size of the melanin based breast stripe has been suggested to be an important indicator of male quality (Norris 1990), although this was never directly shown. Melanin plumage colouration is so called structural colouration and is often suggested to be a trait with a genetic basis due to which it is stable between years and heritable. For instance in sparrows melanin based bib size has been shown to correlate with social dominance and male-male competition (Nakagawa et al. 2007; but see Sánchez-Tójar et al. 2018). Thus, this plumage trait is thought to be indicative of indirect benefits, as well as, through dominance effects, direct benefits in the form of for instance territory quality (Norris 1990; Norris 1993).

The yellow colour of the breast plumage on the other hand, cannot be synthesised by the birds, and is therefore dependent on the ingestion of carotenoids from food (McGraw 2006). Especially the consumption of caterpillars during moult is thought to increase the intensity of the yellow breast plumage. This plumage ornament appears to be indicative of direct benefits in the form of higher foraging ability. More yellow males are thought to be better at finding food (Senar et al. 2008; García-navas et al. 2012) and thus better at provisioning offspring either through a higher visit rate or through the provisioning of higher quality prey types (Senar et al. 2002; García-navas et al. 2012; Pagani-Núñez and Senar 2014). However, not all studies find this correlation between plumage coloration and feeding rates (Hegyi et al. 2007). 


\section{Personality}

Personality describes the individual consistency of suits of behavioural traits (van Oers and Naguib 2013), which in part has a genetic basis (van Oers \& Sinn 2013, Laine \& van Oers 2017). In great tits, a commonly used operational measure of personality is the reaction to a novel environment as a measure of exploratory behaviour (Drent et al 2003), which is correlated with other behavioural characteristics such as boldness and aggressiveness. Personality is known to influence survival and reproductive success (Both et al. 2005; Smith and Blumstein 2007; Dingemanse and Reale 2013) and is thus expected to be selected upon (Dingemanse et al. 2004). For instance, fast exploring female great tits have smaller fledglings and a lower probability of successful breeding (Both et al. 2005), and while fast exploring males were found to obtain a higher quality territory (Both et al. 2005; Scales et al. 2013), aggressive males are also known to provision less often (Mutzel et al. 2013). Therefore, the personality of a mate may have a direct effect on reproductive success and hence may be subject to mate preferences (Schuett et al. 2010). Additionally, studies are beginning to indicate that individuals differ in their preferences for a mate depending on their own personality. Indeed, in a number of species mate preferences or choice for behavioural similarity have been reported (Schuett 2008; Kralj-Fišer et al. 2013; Class et al. 2014). And especially pair compatibility on basis of personality is expected to affect parental investment and coordination (Both et al. 2005; Schuett et al. 2010; Patrick and Browning 2011; Patrick et al. 2012). Since great tit pairs rear their offspring together we expected that behavioural compatibility, depending on the combination of personalities may affect their parental care and with it reproductive success. We explored these effects of personality and compatibility on reproductive success in chapter 5 .

\section{Genetic traits}

Heterozygosity, or the genetic variability within an individual, is known to be positively correlated with fitness aspects (reviewed in Chapman et al., 2009), such as reproductive success (Foerster et al. 2003; Seddon et al. 2004; Ortego et al. 2007), survival (Coltman et al. 1998; Hansson and Westerberg 2002; Da Silva et al. 2009), immunocompetence (AcevedoWhitehouse et al. 2002; Hawley et al. 2005; Reid 2007) and parasite resistance (MacDougall-Shackleton et al. 2005; Rijks et al. 2008; Voegeli et al. 2012). An individual can thus potentially increase its fitness by selecting a mate with which it would produce more heterozygous and 'fitter' offspring (Tregenza and Wedell 2000; Foerster et al. 2006; Tomiuk et al. 2007; Szulkin et al. 2009). By finding an unrelated, or genetically dissimilar mate, offspring heterozygosity can be increased and the negative effects of inbreeding avoided when inbreeding depression is larger than outbreeding depression (Van de Casteele et al. 2003; Szulkin et al. 2007). Moreover, although most studies focus on these indirect effects, genetic traits can have direct effects on reproductive success as well, especially in species with biparental care (Ferrer et al. 2015). While in great tits the effects 
of heterozygosity on mate preference and reproductive success have not been studied very extensively, in blue tits heterozygous females have been found to lay larger clutches (Foerster et al. 2003), and heterozygous males have been shown to have better territories (Seddon et al. 2004; Ryder et al. 2010), and to feed their offspring more often (García-navas et al. 2009). This suggests that in great tits these traits may also affect reproductive success and may therefore play a role in mate preferences. In chapter 3 we explore the effects of genetic characteristics such as heterozygosity and relatedness on preference choice and reproductive success.

\section{Outline of this thesis}

The overall aim of this thesis was to assess individual differences in preferences in great tits and the role of compatibility in mate preferences, mate choice and reproductive success. To test mate preferences, in chapter 2 I first studied how great tits evaluate potential partners, since this may affect results and conclusions drawn from these preference tests. Using these preference tests, I examine in chapter 3 how preferences for traits in a mate may differ depending on the choosers own traits, in this case in particular genetic traits such as heterozygosity and relatedness, how these preferences are reflected in choice, and how this compatibility gives both indirect and direct benefits to reproductive success. In chapter 4 I study these preferences on an even more detailed level, where I test for individual differences in preferences for certain traits in a mate and estimate individual preference functions for several traits. On the basis of these preference functions I subsequently calculate a measure of partner attractiveness - the extent to which parents have the trait values that are preferred by their partner, and test for reproductive benefits of having an attractive mate. In chapter 5 I examine the role of personality and behavioural compatibility in parental care and provisioning behaviour and how behavioural compatibility may influence reproductive success. Finally, in chapter $\mathbf{6}$, the general discussion, I draw general conclusions from all findings in previous chapters and discuss them in a wider context. I conclude with identifying gaps in our current knowledge and potential for future research 



\section{Chapter 2}

Measuring mate preferences: Absolute versus comparative evaluation of potential partners.

Lies Zandberg, Camilla A. Hinde, Kees van Oers 


\begin{abstract}
Quantifying the direction and strength of mate preference is essential to better understand the process of sexual selection. Experimental designs, however, often fail to consider how individuals evaluate and compare the available options, which may affect the results significantly. Preferences are often assumed to be absolute, assigning a fixed, absolute value to a cue or potential partner they encounter. However, in reality, individuals may compare the available options, in which case the social context plays an essential role in the preference for each potential partner. Here we investigate the importance of considering the choosers' evaluation process in mate preference tests. Using a study of wild great tit mate preferences for heterozygosity as a case study, we tested whether individuals use absolute or comparative mate preferences. We analysed how the perceived average attractiveness and the variation in attractiveness of the group of potential mates, they were presented with, affected the measured preference functions. With such knowledge of how a study species encounters and evaluates potential mates, it is possible to choose the most appropriate experimental design and analysis to obtain reliable measurements of mate preferences. The ability to more accurately quantify preference is expected to increase our understanding of mate preferences, mate choice, and ultimately sexual selection.
\end{abstract}




\section{Introduction}

Mate choice is the process in which the effects of traits expressed by one sex lead to nonrandom mating (Kokko et al. 2003). By choosing a suitable mate, individuals are expected to benefit either in terms of direct fecundity or survival benefits, or in terms of indirect benefits such as compatible or good genes. To be able to maximise benefits, individuals need to correctly assess the available options and, according to their internal assessment rules, select a suitable mate. These internal assessment rules can be characterised as preference functions, in which the mating response varies with the value of a trait (Wagner 1998; Edward 2015). Since mate preferences in combination with external factors such as sampling strategy, local availability and competition lead to the ultimate choice of a mate, they are a vital part of understanding how sexual selection works. In theoretical models on sexual selection mate preferences are often assumed to be absolute, assigning a fixed, value to a certain trait or potential partner they encounter (Real 1990; Jennions and Petrie 1997). To do this, individuals have to assess each courter and remember the information about each of these potential mates before they can make a decision (Bateson and Healy 2005). This is often the case in species where choosers commonly only encounter potential mates one at a time. In this case the response to a certain trait value is directly linked to an internal preference, and the social context does not change these results (Moore and Moore 1988). This cognitive process of information processing is relatively complicated, especially when multiple cues indicating quality are combined (Candolin 2003).

In reality, to choose faster and more efficiently, individuals may instead compare the available options relative to each other, without assessing the absolute trait value (Bateson \& Healy, 2005). This so called comparative choice is assumed to be a shortcut that involves less cognitive processing than absolute choice. In this case the attractiveness of one individual depends on who it is being compared to, rather than the absolute value of its quality (Bateson \& Healy, 2005). Since in many species potential mates are encountered simultaneously (Duval and Kempenaers 2008; Reaney 2009), this would allow for a direct comparison of the courters (Castellano et al. 2012).

Depending on a species' memorising abilities, if different individuals or groups are encountered at different time points, there may even be the potential to compare between individuals or groups over different time points. Especially in species where males are distributed scarcely, for instance over different territories, the ability to remember the attractiveness of potential mates that were encountered previously, will enable choosers to compare between them (Dale and Slagsvold 1996). Thus, when individuals use a comparative evaluation process, the measured response or attractiveness of a potential mate may vary depending on the social context in which it is encountered (Royle et al. 
2008; Locatello et al. 2015). This distinction between absolute and comparative choice may not be as black and white as it seems, which has been shown for a number of species. For instance, sailfin mollie females (Poecilia latipinna) show preferences for larger sized males when sequentially presented with a single male, but when alternatives were presented simultaneously much stronger size preferences were observed (Maclaren and Rowland 2016). This suggests that when given the opportunity to compare, females can use both absolute evaluation and comparative evaluation and thus the experimental design can influence the (strength of) measured preferences. Commonly, the evaluation process used by the choosers is not considered, and this variation in mating responses caused by the social context is often assumed to be an assessment error or noise in the data. Perhaps more importantly, depending on which evaluation process choosers use, not all methods of measuring mate preferences may be equally appropriate.

An important way in which studies measuring preferences differ lies in the number of options or stimuli the chooser is presented with: the choice design (Dougherty and Shuker 2014). Tests can use a no-choice or a choice design (Wagner 1998). In a no-choice design the chooser is presented with one stimulus at a time, often showing different stimuli sequentially. In contrast, in a choice design, choosers are presented with more than one stimulus simultaneously allowing for comparative evaluation of stimuli (Edward 2015). Typically, 2 stimuli are presented to measure a directional preference, but by measuring, for example, 4 or more stimuli it is also possible to measure disruptive or stabilising preference functions. Preferably these tests are repeated with different groups of stimuli. If choosers use an absolute evaluation process, the value of a trait is directly linked with the internal preference and thus the social context or the possibility for direct comparison does not play a role in measurements of preference. Thus, presenting stimuli sequentially or simultaneously will not affect preference measurements. However, when choosers only use direct comparison for mate selection, a sequential presentation of single stimuli may give aberrant results. In that case all options are evaluated equally if individuals cannot compare. Alternatively, if individuals are able to compare previously assessed stimuli with current stimuli (comparison over different time points), order effects can influence the results. Additionally, when using multiple choice tests, the social context may still be important to consider. Differences between stimulus groups, such as mean trait values and variation in trait values may add noise to the results, making it more difficult to discern preference patterns. Therefore, taking into account the effects of the social context in analysing the data can significantly improve preference functions and conclusions drawn from the data. Moreover, when individuals can compare over different time points, group characteristics of a previously measured stimulus group may have carry-over effects for the measured preferences into a subsequent test. Thus, depending on how a study species 
evaluates (absolute, comparative or a combination of both), and how well it is able to compare between contexts (only direct comparison or also comparison over different time points), different methods of measuring and analysing preferences should be used.

Here we study the importance of considering the choosers' evaluation process in mate preference tests. As a case study, we use a study on great tit mate preferences for heterozygosity (chapter 3 ). In this study, wild great tits were tested for their preferences for mate heterozygosity using a 6-choice setup. Using this dataset, we tested whether wild great tits use absolute or comparative mate preferences and how the perceived attractiveness and variation in potential mates affects preference functions. By studying the effects of between stimulus group differences in average stimulus group heterozygosity and variation in heterozygosity on the total association time (total time spent with any of the stimulus birds in a given test) and on the preference slope per test (regression of trait value and association time in a given test) we were able to test how great tits evaluate potential partners. Additionally, by analysing the effects of previously presented stimulus group characteristics we test whether previous experiences have an effect on current preferences, and thus whether choosers also compare over different time points. We hypothesize that depending on the evaluation process used by a chooser, the group characteristics of the stimulus groups (mean attractiveness and variation in attractiveness) choosers are presented with, will have different effects on the preference (total association time) and the preference slope (for hypotheses see Box 1). 


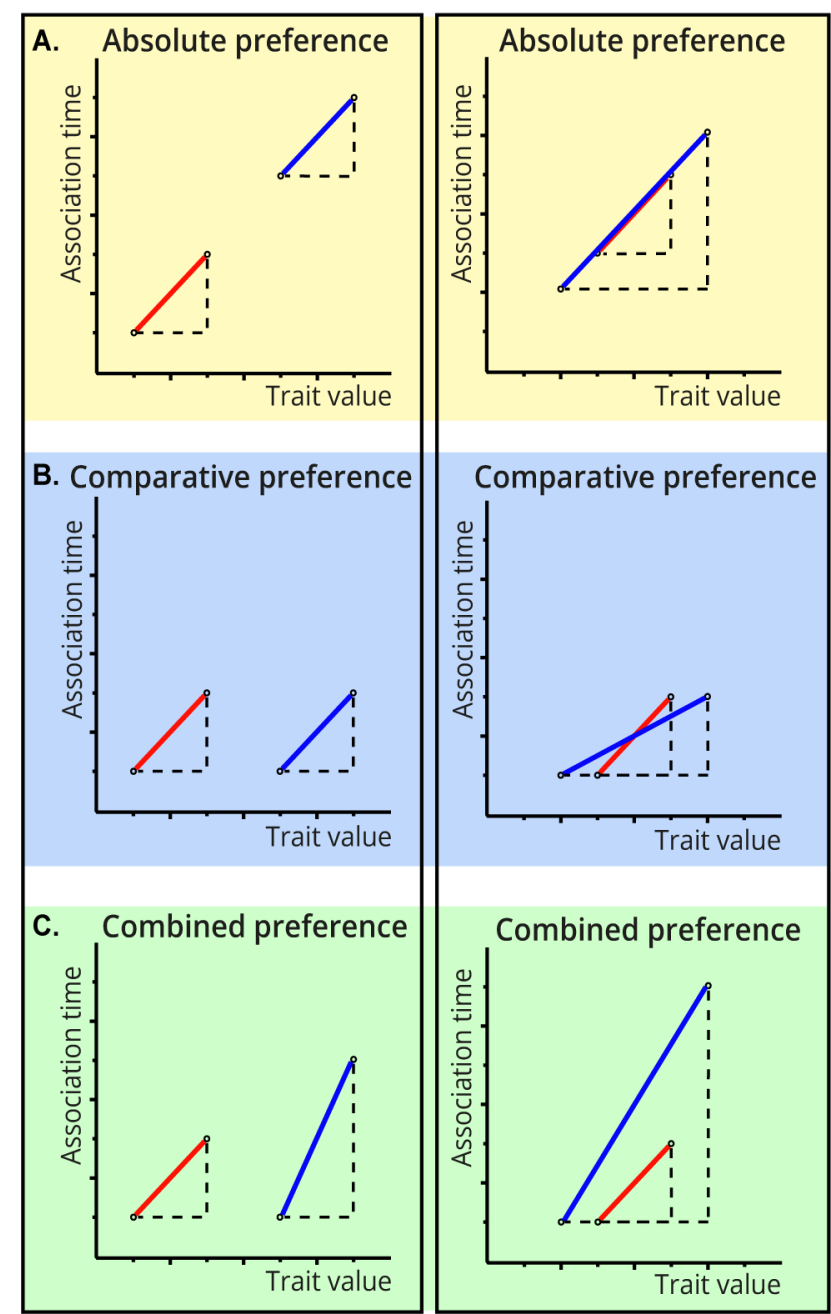

Box 1 - For different evaluation processes, the variation in total association time and slope in response to differences in mean trait values and variation in trait variation. How do the total association time and slope vary between stimulus sets, which differ in mean trait values and variation in trait values, depending on the different evaluation processes? Here we assume positive preferences for a hypothetical trait. A) In the case of absolute choice individuals will assign a fixed attractiveness value to a specific trait value, regardless of the available options. Thus, we expect the total association time during a test to increase with increasing mean attractiveness, and that, regardless of the variation in the attractiveness within the stimulus group, the preference slopes will stay constant. B) In the case of comparative choice, we assume that individuals will assess each stimulus option in comparison to the other five options. Thus, we expect them to have a fixed total association time (which is dependent on their motivation to choose, rather than the available options) and to not increase their total association time depending on the mean attractiveness. However, with an increase in attractiveness variation we expect choosers to have a lower preference slope, as the same total association time is now divided over a larger range of trait values. $C$ ) This choice process may be somewhere on the gradient between absolute and comparative choice. In this case, both the slope and total association time increase with higher attractiveness. Choosers may spend most time with the most attractive option in the stimulus set, but when this most attractive option is extremely attractive they may be even more motivated to spend time with this particular potential mate. Similarly, with increasing variation the most attractive individual may seem even more attractive compared to the other available options causing them to spend a higher amount of time with this individual, increasing the total association time and preference slope. Additionally, we tested whether individuals showed betweentest comparisons. We analysed whether a previous experience would affect the preference measured in a current test. We hypothesised that if individuals remember the previously presented stimulus groups and compare them to the current stimulus group, that the total association time and/ or the slope for the current stimulus group would change depending on the mean trait value and the variation in trait values of the previously presented stimulus group. 


\section{Methods}

\section{Preference tests}

For all details on the methods of the preference tests see chapter 3 . We performed preference experiments using wild great tits originating from six different field sites, to minimize the chance the stimulus birds (chosen birds) were familiar with the focal birds (choosers). We carried out the mate preference tests at the NIOO-KNAW facilities. All tests took place in a test room $(4.0 \times 2.4 \times 2.5 \mathrm{~m})$ with white walls, and high-frequency fluorescent lights to mimic natural lighting conditions. We tested individuals mate preferences using a carrousel shaped six-choice chamber (chapter 3). As unmated great tits pair up throughout the winter period (Culina 2014), we tested the birds for their mate preferences in the months of January, February and March. During the winter great tits often forage in large foraging flocks, while keeping local dominance in their breeding territories. Encountering 6 birds of the opposite sex is therefore not unrealistic for this species. Moreover with the six-choice test it is possible to measure individual directional preferences as well as quadratic (stabilising or disruptive) preferences (Edward 2015).

Each focal bird was tested with 3 different groups of 6 stimulus birds. In total we tested 69 focal females and 70 focal males for their preference with a total of 42 groups of 6 stimulus birds ( $N$ = 252).

The setup consisted of a central zone for the focal bird and 6 stimulus cages placed around the central zone (Figure 2.1). The stimulus cages were separated from the central area with transparent UV transmitting panels, to allow visual contact between the focal bird and a stimulus bird. All birds were within auditory range of each other. In the middle of the central focal

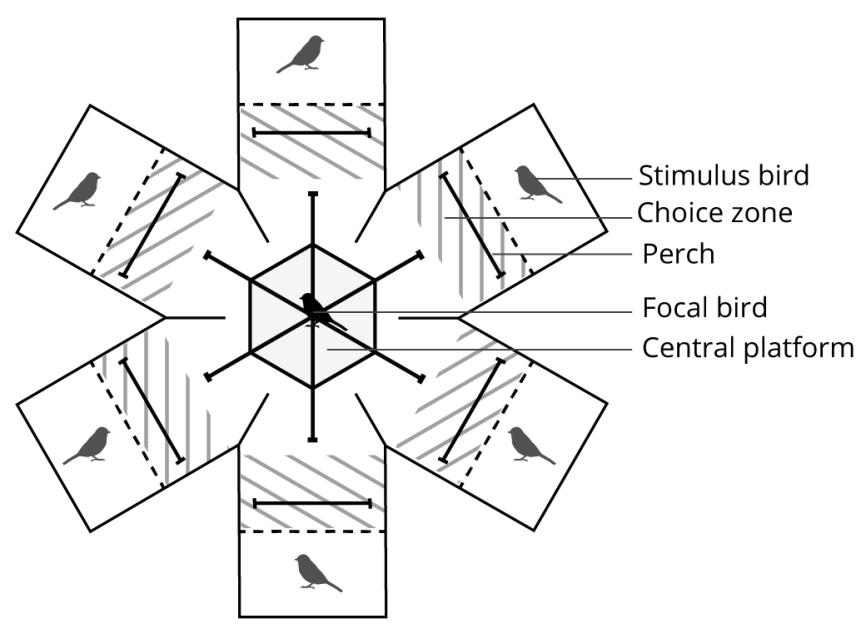

Figure 2.1 - Experimental setup mate preference test. Birds were tested for their preference in a six-choice test room. From the hexagonal central platform the focal bird could observe all stimulus birds, whereas from the perches in the choice zone only one stimulus bird was visible. Time spent in each of the choice zones was measured. compartment we placed a hexagonal platform to prevent visual contact between the stimulus birds. On top of this, we placed a stick with 6 branches of equal length pointing in the direction of the stimulus cages, from which the focal bird could observe all stimulus birds simultaneously. Two perches were present in front of every 
stimulus cage, for the focal bird to sit on in proximity of the stimulus bird (choice zone). From this position it could only observe that specific stimulus bird. The movements of the focal bird were recorded using a central camera pointing down from the ceiling (Panasonic WV-CP500). We analysed the video using EthoVision XT software (Noldus Information Technology), to calculate the time that the focal bird spent in each choice zone.

For every test, we placed an individual bird in each of the six stimulus cages and gave them 15 minutes to habituate. After introducing the focal bird into the setup, we started the recording, which lasted 45 minutes. Each focal bird was tested with 3 groups of different stimulus birds (groups A, B and C), of which one group was repeated (order of testing: ABCA). Every stimulus bird participated in 13 or 14 trials with 10 focal birds, divided over 4 mornings and afternoons over a period of six days. We randomised the positions of the stimulus birds within a group. We composed every group of stimulus birds blind with regard to their traits (natural variation). From the videos, we calculated the time spent in front of every stimulus bird, which is commonly used as a measure of preference. In numerous species, association time in a choice test has been shown to predict courtship behaviours or pair formation (Clayton 1990; Hill 1990; Mays and Hill 2004; Witte 2006; Lehtonen and Lindström 2008; Dechaume-Moncharmont et al. 2011; Jeswiet and Godin 2011) and correlates with reproductive success (Drickamer et al., 2003).

Trials in which the focal bird did not visit any of the stimulus birds $(\mathrm{N}=51)$ were excluded from the analysis.

\section{Heterozygosity}

We genotyped all birds that participated in the mate preference experiments (344 individuals) across 17 polymorphic microsatellite markers. Blood samples of 9 birds tested in the mate preference tests were missing and thus omitted from the genotype analysis.

We used homozygosity by locus (HL; Aparicio et al., 2006) to estimate individual genome wide genetic diversity and calculated this measure for all genotyped individuals with the $R$ package GENHET (Coulon 2010). Because HL represents homozygosity instead of heterozygosity we transformed the HL values into an estimate of heterozygosity by calculating the complement of HL (1-HL). Full details on marker-characteristics and the genetic analyses can be found in chapter 3 .

\section{Statistical analyses}

To test what type of evaluation method great tits used during our experiments: absolute, comparative or a combination of both, we tested their responses in the preference tests against our hypotheses (see box 1). We tested the effects of between stimulus group differences in average heterozygosity and variation in heterozygosity on the total 
association time (total cumulative time spent with any of the stimulus birds in a given test) and on the preference slope per test (regression of trait value on association time in a given test). From previous research we know that in great tits heterozygosity is a trait that plays an important role in mate preferences (chapter 3 ). Therefore, we use this trait to measure preference responses. However, since individuals differ in their preference, both in direction and in strength, high heterozygosity levels have been found to be attractive to some, but unattractive to others. Consequently, also the effects of mean heterozygosity levels within a stimulus set may have opposite effects on individuals depending on their preference. To overcome this in our analysis we analysed how the total association time changed with between stimulus group differences in mean heterozygosity levels, depending on their previously measured preference (which is based on within stimulus group effects of stimulus heterozygosity on the association time spent with each stimulus individual). To make the difference between 'effects of within-group differences in heterozygosity' (preference) and 'effects of between-group differences in heterozygosity' more intuitive we will refer to the mean attractiveness and variation in attractiveness in stimulus groups respectively. For birds that have increased association times with stimuli with a high heterozygosity level within a group (a positive preference), groups with a high mean heterozygosity level are referred to as attractive groups, and vice versa. To obtain these overall preference slopes for each individual, we ran linear models for each individual, with the logged association time as the dependent variable, stimulus heterozygosity as the explanatory variable and corrected for other traits such as body condition. We included breast stripe size and yellowness (see appendix A for details on these measurements) as the explanatory variables and test identity as the random effect. Mean stimulus heterozygosity levels were calculated as the mean of heterozygosity levels of the six stimulus birds in a given stimulus set. We calculated the variation in stimulus heterozygosity levels by taking the standard deviation of the heterozygosity levels of the six birds in the stimulus set.

We first tested the correlations between the slope, the total association time and number of visited birds in tests. We found no significant correlation between the preference slope and the total association time in a test $(\mathrm{r}=0.02, \mathrm{P}=0.73)$, or between the slope and the number of birds visited in a test $(\mathrm{r}=-0.02, \mathrm{P}=0.73)$. However, when the number of visited birds in a test was higher, the total association time for stimulus birds in that test was also higher $(\mathrm{r}=0.75, \mathrm{P}<0.001)$. Additionally, we tested the correlation between mean stimulus trait and the variation in stimulus traits. Stimulus sets with a higher mean heterozygosity also had higher variation in their heterozygosity levels $(r=0.39, \mathrm{P}=0.02)$. Despite the correlation between these variables, having both in the same model did not cause problems of collinearity. Moreover, by having both in the same model, we were able to 
distinguish between the effects correlated with mean heterozygosity and variation in heterozygosity.

We tested how the total association time and the preference slope changed depending on the trait values of the stimulus individuals within a stimulus set (whilst taking the preference of the individual into account). All continuous variables in the models were mean centred. For all models described we used backwards elimination on the basis of the estimated confidence intervals and the corresponding $\mathrm{P}$ values to obtain the minimal adequate models. Non-significant terms were deleted stepwise, starting with the highest order interactions and/or the least significant term $(\mathrm{P}<0.05)$.

\section{Total association time}

First we tested how the total association time changed depending on between-group differences in mean heterozygosity level and the variation in heterozygosity levels within a stimulus set. For this we ran a linear mixed model with the logged total association time as dependent variable. The total association time was calculated as the sum of the time spent in the choice zone of each of the stimulus birds. As fixed effects we added the mean stimulus group heterozygosity, the within stimulus group variation in heterozygosity and the overall preference slope for heterozygosity of each focal individual. Since the effects of changes in mean stimulus values will differ depending on an individuals' overall preferences, we added the interaction between mean stimulus heterozygosity and the overall preference slope for heterozygosity. To account for the multiple tests we ran with each individual we included a random effect for focal individual, and a random effect for stimulus group identity.

Subsequently we ran two extra models to test whether a change in total association time could be explained by a change in the number of stimulus birds that was visited, or by a change in the average time spent with each visited bird. First we ran a binary generalised mixed model with the number of visited birds out of the total of 6 birds as the dependent variable (numerator $=$ number of visited birds, denominator $=6-$ number of visited birds), and the mean stimulus heterozygosity, the variation in heterozygosity, the overall preference slope for heterozygosity of each focal individual and the interaction between the mean stimulus heterozygosity and the overall preference slope of each focal individual as fixed effects. Second, we ran a linear mixed model with the average association time of only the stimulus birds that were visited as a dependent variable. In this model we added the number of visited birds, the mean stimulus heterozygosity, the variation in heterozygosity, the overall preference slope of each focal individual and the interaction between the mean stimulus heterozygosity and the overall preference slope for heterozygosity of each focal individual as fixed effects. Both models had a random effect 
for focal individual and stimulus group identity. Using these two models we were able to tease apart whether a change in total association time could be explained by a change in the number of stimulus birds that was visited, or by a change in the average time spent with each visited bird

\section{Slopes}

We then tested how the preference slopes changed depending on the mean stimulus group heterozygosity level and the variation in heterozygosity levels within a stimulus group. To calculate the preference slopes per test we ran a linear regression for each focal individual and for each test, with the logged association time as the dependent variable and stimulus heterozygosity, body condition, breast stripe size and yellowness as the explanatory variables. From each model we extracted the coefficient of the preference slope for heterozygosity levels and used these as the dependent variables for the subsequent models. We then tested how the steepness of these individuals' slopes were affected by the mean heterozygosity value of the stimulus group and the variation in heterozygosity levels in that stimulus set. Under combined preferences (combination of absolute and comparative), we expect that slopes may change when stimulus groups are more attractive, however, which stimulus groups are more attractive again depends on the direction of the preference. For instance, for birds with a positive preference we expect higher slopes for stimulus groups with higher mean trait values, whereas for birds with a negative preference we expected slopes to be lower with high mean trait values. To overcome this, we filtered the dataset for birds with a positive overall slope (slope estimate $>3$ ) and birds with a negative overall slope (slope estimate <-3). We then ran a linear mixed model in which we tested the absolute value of the test slope as the dependent variable, with the mean heterozygosity level and the variation in heterozygosity levels of the stimulus set as explanatory variables. We added focal identity and stimulus group identity as random effects. 


\section{Results}

\section{Total association time}

The total association time spent with all stimulus birds, depended on both the social context stimuli were presented in and on the preference of the chooser (Figure 2.2). Birds spent less time in association with stimulus birds from groups with a high mean attractiveness. Birds for which high heterozygosity levels are more attractive (i.e. that showed a positive correlation between association time and stimulus heterozygosity levels within a test), showed a negative correlation between total association time and the mean stimulus group heterozygosity levels between different tests (mean stimulus HL * overall preference slope, LMM: $\mathrm{t}-328=-2.73$, $\mathrm{P}=0.007$; Table 1). Vice versa, birds for which low heterozygosity levels are more attractive showed a positive correlation between the total association time and the mean stimulus group heterozygosity levels between different tests.

The fact that individuals spent less time in association when confronted with attractive groups had two different causes. Firstly, focal birds visited a higher number of birds in unattractive groups than in attractive groups (mean stimulus HL * overall preference slope, Binomial GLMM: $Z=-2.57$, $\mathrm{P}=0.01$ : Table 2, Figure 2.3a). And secondly, when analysing the mean association time per visited bird, birds spent more time per

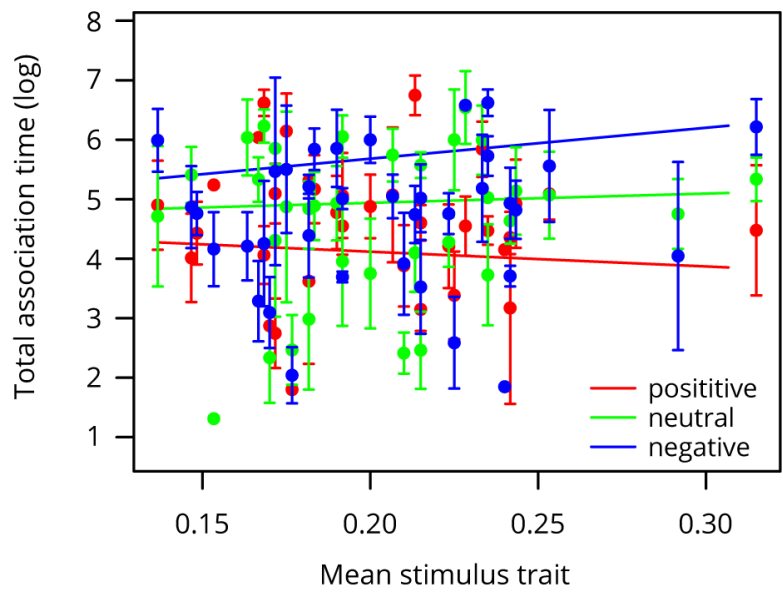

Figure 2.2 - Effects of mean stimulus group heterozygosity on total association time spent per test were dependent on the overall preference of the choosing birds. Choosers spent more time visiting birds when presented with unattractive groups. Models contained overall preference slopes as continuous variable, for visualisation purposes here we indicate positive, neutral and negative overall preferences with different colours. Error bars indicate mean $\pm S E$ for focal birds with positive (red), negative (blue), and stimulus birds in unattractive groups. neutral (green) preferences for that trait. (mean stimulus $\mathrm{HL}{ }^{*}$ overall preference slope, LMM: $\mathrm{t}=-2.44, \mathrm{P}=0.02$ : Table 2, Figure 2.3b). Additionally, when visiting more stimulus birds, focal birds also spent more time per visited bird (number of visited birds, LMM: $\mathrm{t}_{301.6}=10.05, \mathrm{P}<0.001$ ) and when stimulus groups had a higher variation in $\mathrm{HL}$, focal birds spent less time per visited bird (variation in stimulus HL, LMM: $\mathrm{t}_{27.4}=-2.34, \mathrm{P}=0.03$ ). 
Table 1: Minimal adequate model for LMM, testing whether the total association time was dependent on the mean and variation in stimulus heterozygosity $(\mathrm{HL})$. Table consists of all variables tested in the linear mixed model with the total association time as the dependent variable ( $N=398)$. Random effects for focal identity (var $\pm S D: 0.66 \pm 0.81$ ) and stimulus group identity (var $\pm S D: 0.45 \pm 0.67$ ) were included in the model. Given is the estimate, the degrees of freedom (df), the test statistic ( $t$ value) and the significance (P value). Significant terms are indicated in bold.

\begin{tabular}{lcccc}
\hline & Estimate & df & t value & P value \\
\hline Minimal adequate model & & & & \\
Intercept & 4.63 & - & - & - \\
Mean stimulus HL & 2.24 & 24.5 & 0.70 & 0.49 \\
Preference slope (HL) & -0.003 & 104.7 & -0.18 & 0.85 \\
Mean stimulus HL : preference slope (HL) & -0.89 & 328.0 & -2.73 & $\mathbf{0 . 0 0 7}$ \\
\hline Dropped terms & & & & \\
Mean stimulus HL (previous group) : preference & -0.25 & 315.3 & -0.67 & 0.50 \\
slope (HL) & -0.12 & 227.30 & -0.04 & 0.97 \\
Variation stimulus HL (previous group) & -6.08 & 26.90 & -1.37 & 0.18 \\
Variation stimulus HL & & & & \\
\hline
\end{tabular}
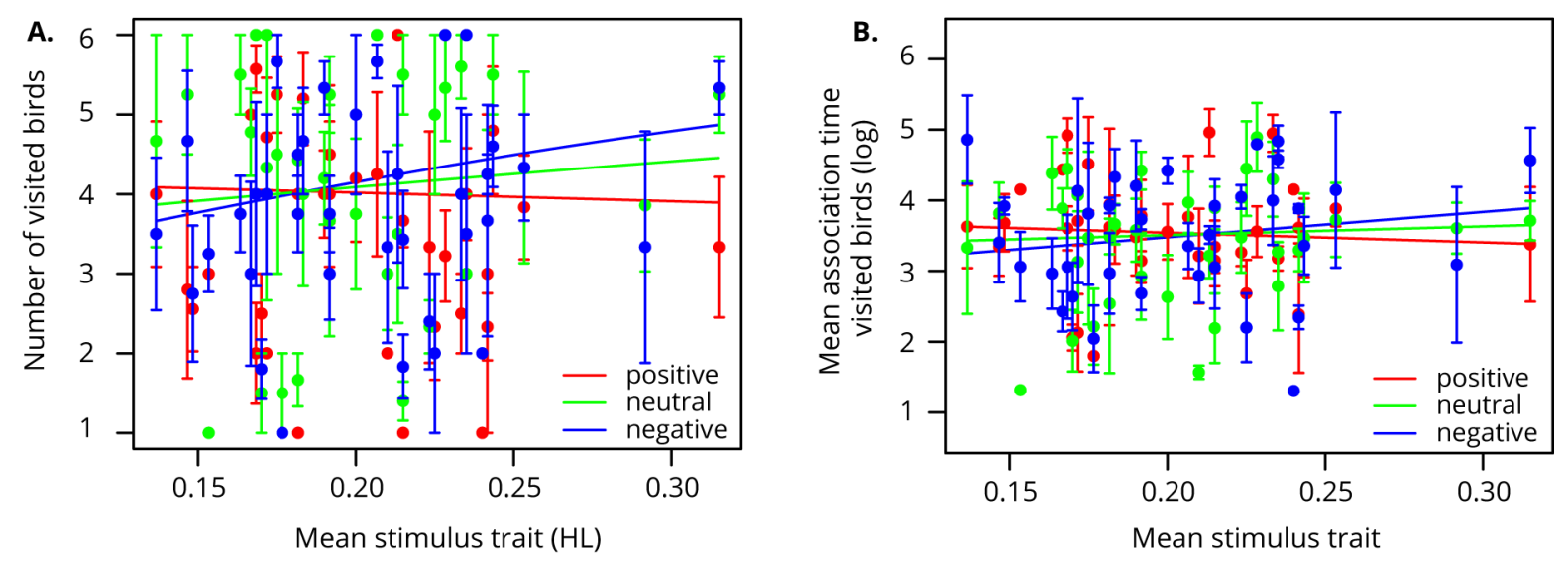

Figure $2.3-A)$ Effects of mean stimulus group heterozygosity on the number of birds visited per test were dependent on the overall preference of the choosing birds. Choosers visited more birds in unattractive groups. B) Even when taking into account the number of birds visited, the effect of mean stimulus group heterozygosity on the time spent per bird depended on the overall preference of the bird. Choosers spent more time per bird in unattractive groups. Models contained overall preference slopes as continuous variable, for visualisation purposes here we indicate positive, neutral and negative overall preferences with different colours. T bars (mean $\pm S E$ ) indicate for each stimulus group the number of visited birds (A) or mean association time per visited bird (B) for focal birds with positive (red), negative (blue), and neutral (green) preferences for that trait. 
Table 2. Minimal adequate model for LMM testing whether the number of visited birds was dependent on the mean and variation in stimulus heterozygosity $(\mathrm{HL})$. Table consists of all variables tested in the binary mixed model with the number of visited birds per test (numerator = number of visited birds, denominator $=$ number of birds not visited) as the dependent variable $(N=398)$. Random effects for focal identity (var $\pm S D: 1.06 \pm 1.03$ ) and stimulus group identity (var $\pm S D: 0.65 \pm 0.81$ ) were included in the model. Given is the estimate, the degrees of freedom (df), the test statistic ( $z$ value) and the significance (P value). Significant terms are indicated in bold.

\begin{tabular}{lccc}
\hline & Estimate & Z value & P value \\
\hline Minimal adequate model & & & \\
Intercept & 0.13 & - & - \\
Mean stimulus HL & 3.19 & 0.86 & 0.39 \\
Preference slope HL & 0.14 & 2.22 & 0.02 \\
Mean stimulus HL * preference slope HL & -0.75 & -2.57 & $\mathbf{0 . 0 1}$ \\
\hline Dropped terms & & & \\
Mean stimulus HL (previous group) * preference slope (HL) & -0.57 & -1.57 & 0.12 \\
Variation stimulus HL & -1.59 & -0.30 & 0.76 \\
Variation stimulus HL (previous group) & -2.08 & -0.71 & 0.48 \\
\hline
\end{tabular}

Table 3. Minimal adequate model for LMM testing whether the time spent per visited birds was dependent on the mean and variation in stimulus heterozygosity (HL). Table consists of all variables tested in the linear mixed model with the time spent per visited bird per test as the dependent variable $(N=398)$. Random effects for focal identity (var $\pm S D: 0.15 \pm 0.38$ ) and stimulus group identity (var $\pm S D$ : $0.04 \pm 0.21$ ) were included in the model. Given are the estimate, the degrees of freedom (df), the test statistic ( $t$ value) and the significance ( $P$ value). Significant terms are indicated in bold.

\begin{tabular}{lcccc}
\hline & Estimate & df & t value & P value \\
\hline Minimal adequate model & & & & \\
Intercept & 3.51 & - & - & - \\
Mean stimulus HL & 1.66 & 24.5 & 1.09 & 0.29 \\
Preference slope HL & 0.01 & 101.8 & 0.52 & 0.60 \\
Variation stimulus HL & -4.59 & 27.4 & -2.34 & $\mathbf{0 . 0 3}$ \\
Visited stimulus birds & 0.28 & 301.6 & 10.05 & $<\mathbf{0 . 0 0 1}$ \\
Mean stimulus HL * preference slope HL & -0.57 & 364.8 & -2.44 & $\mathbf{0 . 0 2}$ \\
\hline Dropped terms & & & & \\
Mean stimulus HL (previous group) * preference slope & -0.03 & 339.9 & -0.10 & 0.92 \\
(HL) & & & & \\
Variation stimulus HL (previous group) & -0.28 & 1.98 & 106.9 & 0.89 \\
\hline
\end{tabular}




\section{Slopes}

There was no effect of mean stimulus attractiveness nor of the number of stimulus individuals visited on the individual preference slopes. Only the variation in attractiveness has an effect on the steepness of the preference slopes: the slopes were lower for groups with high variation (variation stimulus HL, LMM, positive slopes: $\mathrm{t}_{21.85}=-2.50$, $\mathrm{P}=0.02$, table $4 \mathrm{~A}$; negative slopes: $\mathrm{t}_{21.60}=-2.78, \mathrm{P}=0.01$, table $4 \mathrm{~B}$ ).

Table 4. Minimal adequate model for LMM testing individual preference slope was dependent on the mean and variation in stimulus heterozygosity $(\mathrm{HL})$. Table consists of all variables tested in the linear mixed model with the absolute slope per test as the dependent variable were included in the model. Given is the estimate, the degrees of freedom (df), the test statistic (t value) and the significance (P value). Significant terms are indicated in bold. A. Model for all birds with negative preferences $(N=74)$, random effects for focal identity (var $\pm S D: 0.16 \pm 0.40$ and stimulus group identity (var $\pm S D: 0.26 \pm 0.51$ ) B. Model for all birds with positive preferences $(N=138)$, random effects for focal identity (var $\pm S D: 0.08 \pm 0.28)$ and stimulus group identity (var $\pm S D: 0.02 \pm 0.14$ ).

\begin{tabular}{lcccc}
\hline A & Estimate & df & t value & P value \\
\hline Minimal adequate model & & & & \\
Intercept & 2.40 & 21.62 & 16.44 & - \\
Variation stimulus HL & -9.56 & 21.85 & -2.50 & $\mathbf{0 . 0 2}$ \\
\hline Dropped terms & & & & \\
Mean stimulus HL (previous group) * preference slope (HL) $^{*}-0.14$ & 46.52 & -0.39 & 0.70 \\
Mean stimulus HL * preference slope HL & -1.45 & 55.02 & -1.54 & 0.13 \\
Variation stimulus HL (previous group) & 0.92 & 62.22 & 0.22 & 0.82 \\
\hline
\end{tabular}

\begin{tabular}{lcccc}
\hline B & Estimate & df & t value & P value \\
\hline Minimal adequate model & & & & \\
Intercept & 2.15 & 22.36 & 26.98 & - \\
Variation stimulus HL & -6.03 & 21.60 & -2.78 & $\mathbf{0 . 0 1}$ \\
\hline Dropped terms & & & & \\
Mean stimulus HL (previous group) ${ }^{*}$ preference slope (HL) & -0.27 & 85.78 & -0.75 & 0.46 \\
Mean stimulus HL * preference slope HL & -0.51 & 128.62 & -0.77 & 0.44 \\
Variation stimulus HL (previous group) & 3.80 & 52.85 & 1.63 & 0.11 \\
\hline
\end{tabular}




\section{Discussion}

Here we show that when experimentally testing mate preferences, the evaluation method used by the study species to assess the potential mates matters. In this case study, wild great tits used a combination of both absolute and comparative choice, indicating that for such evaluation methods the social context affects the response, and with it the measurement of preference functions. Birds spent on average more time visiting less attractive stimulus groups compared to the more attractive stimulus groups. This increased association time can be explained by two different effects. Firstly, focal birds visited more stimulus birds in unattractive groups. And secondly, in those unattractive groups choosers spent more time on average with each of the stimulus bird they visited. Thus, although these birds had a preference for high heterozygosity mates within groups of stimulus birds, they spent more time on average with birds when they were in an unattractive group. Additionally, the variation in attractiveness within groups affected the measured preference slopes. Preferences measured in groups with low variation in attractiveness were stronger than preferences measured in groups with high variation.

We found that great tits respond differently to stimulus groups depending on the group characteristics. Here we found that the preference slopes depended on the variation in trait values, which indicates that the choosers did not attribute a fixed 'preference value' to each trait value. Instead, the attractiveness of each individual depended on the social context it was presented in, indicating a comparative evaluation process. In this species, unpaired individuals form pairs throughout the winter period (Culina 2014), during which they often form large fission-fusion foraging flocks, which makes it very likely that they have the opportunity to assess multiple potential mates at the same time. Since comparative evaluation requires less cognitive processing (Bateson and Healy 2005; Beatty and Franks 2012), we expected great tits to use this shortcut in decision making, and to compare stimuli for mate choice. And indeed, our results show that great tits are able to compare available options and are able to make choices that depend on the availability of potential partners.

Additionally, we found that the total time spent with each stimulus group was affected by the mean stimulus group attractiveness, indicating that the choosers not only compare stimulus individuals, but also have an absolute notion of the attractiveness of each individual. Surprisingly however, choosers did not spend more time with more attractive stimulus groups as we expected. Instead, they spent more time with the stimulus groups that were on average less attractive. Thus, birds for whom large trait values are more attractive, spend less total time in association with all members of the stimulus group when their group mean trait value was higher and vice versa. This could be explained by 
an increase in both the number of stimulus individuals visited and the time spent with each of the stimulus birds separately. When correcting for the number of stimulus individuals visited, a higher variation in trait values decreased the association time per visited stimulus individual. So, while mean attractiveness affected the number of individuals visited, the time spent with each visited bird is higher for on average unattractive groups and groups with little variation in attractiveness. We hypothesize that this is caused by the evaluation time needed to make the correct decision. When all potential mates are relatively attractive, the costs of making the wrong decision may be lower, which may make it easier to decide. And, when all individuals to choose from are relatively unattractive the costs of making a wrong decision are higher. Choosers may therefore be more motivated to make a right decision and spend more time assessing. Similarly, when the variation between the options is high, choice making may be easier, leading to a lower association time per stimulus individual.

In our experiments, the previous group individuals encountered had no effect on the association time or the preference slope of the next group. It seems that the previous social experience did not affect preferences or choice behaviour. In field crickets (Teleogryllus oceanicus), however, females were less responsive to male song when they had previous experience with male song, and responded differently to current song depending on the quality of the previously heard song (Bailey and Zuk 2009). Also female flycatchers (Ficedula hypoleuca) visited several males in their territories before committing to one of these males (in which the likelihood of choosing a male did not depend on the order of sampling potential mates) (Dale et al. 1992; Dale and Slagsvold 1996). These experimental tests on crickets and the visits by flycatchers, however, took place right after each other, whereas our subsequent tests were done one or two days apart. Thus, either great tits do not remember previous experiences with potential mates, or they don't respond correspondingly. Since adult great tits in winter often forage in large fission fusion flocks, they may have a good understanding of the variation and range of trait values and are therefore not affected by these previous experiences.

The simultaneous use of comparative and absolute evaluation of potential mates in preference tests has been found before. In sailfin mollies female preference for larger males was much stronger when they were presented with multiple choice than when potential males were presented sequentially (Maclaren and Rowland 2016). Similarly, lesser wax moth (Achroia grisella) females rejected males below a certain call rate in a sequential test, but when in the possibility to compare between multiple males above this threshold they chose comparatively for the male with the highest call rate (Brandt et al. 2005). These results (see also Castellano et al., 2012 for a further overview), together with our results suggest that choosers may be able to switch flexibly between or combine different 
evaluation methods depending on the opportunity to compare simultaneously. Great tits may have an absolute internal reference about the attractiveness of potential mates, which would be beneficial in environments with very few potential mates or widely spread potential mates preventing simultaneous comparison. In this way, individuals do not have to remember each specific trait value of each potential mate they encountered, which would be a cognitively demanding process, but can mirror it to an internal reference. However, when there is the possibility to compare potential mates, these birds will use comparative evaluation and change their selectivity depending on the social circumstances and the attractiveness of the available mates (Castellano et al. 2012).

If species differ in the use of absolute or comparative evaluation, or the combination of both, then the choice of experimental design may affect the results and conclusions drawn from mate preference experiments (Dougherty and Shuker 2014). If choosers only use absolute choice the study design will not necessarily affect results of preference tests. When choosers only use simultaneous comparative evaluation, sequential presentation of stimuli may give similar responses to each stimulus they are presented with. However, sequential tests may still give results if individuals can remember what they have seen before to compare with the current, or when they use a combination of absolute and comparative preferences. In the first case however, there would be a large order effect in which preferences may differ depending on the attractiveness of the previous options they were presented with (Bailey and Zuk 2009). And in the second case, results would depend on how absolute and comparative evaluation is combined. For instance in red jungle fowl, females (Gallus gallus), when presented with two males with large combs, preferred the male with the largest comb (Zuk et al. 1990). But when presented with two males with small combs, they made no choice or chose randomly, indicating an absolute threshold as well as a comparison of the large-combed males. Although in our case sequential choice experiments would be needed to really know how responses to sequential tests differ from simultaneous presentation of 6 stimuli, our results suggest that the social context in which stimuli are presented may play a large role. This may perhaps also be the reason that measured effect sizes for preferences in sequential and simultaneous choice tests often have the same direction, but are larger in simultaneous choice tests (Dougherty and Shuker 2014). It should be noted, however, that while choosing a test design, apart from the evaluation process, also the ecological relevance should be kept in mind. In species where mates are only encountered sequentially, even though simultaneous test may give directional preferences, if comparison of potential mates is not ecologically realistic, the effect of these comparative preferences on sexual selection may be minimal and are difficult to interpret (Barry et al. 2010; Booksmythe et al. 2011). 
In species where preferences are affected by the social context, differences in the composition of stimulus groups may add noise to the measurement of these preferences functions. The fact that birds in our study spent on average more time with stimulus individuals from unattractive groups could have suggested a negative correlation between trait values and association time with stimulus birds. However, within tests, there is actually a positive correlation between trait values and association time per stimulus bird. This emphasizes the importance of calculating preferences whilst taking into account these within and between test effects of traits.

Here we have found that the social context can affect the behavioural responses to stimulus groups. Thus, by comparing within and between stimulus group effects on the preference measures it is possible to determine how species evaluate potential mates, and how this social context may affect the results. With such knowledge on how a species commonly encounters and evaluates potential mates, it is possible to choose the most appropriate experimental design. And, additionally, knowing how the social context affects preference measures enables the correct analysis to be done. Additionally, the application of statistical transformations of variables, in order to decrease noise in the response data, is likely to improve the chances of finding reliable preference patterns when those are present. More reliable measurements of mate preferences will improve our understanding of mate preferences, mate choice, and ultimately sexual selection. 
Supplement to chapter 2 


\section{Appendix: Methods trait measurements}

\section{Yellow breast plumage intensity}

We took a sample of at least 12-15 yellow breast feathers from all adult birds that were used in the preference tests $(n=391)$ or caught in the breeding season $(n=128$, of which 29 individuals were sampled in both 2014 and 2015). We obtained plumage reflectance measurements from these feather samples using the method of Quesada and Senar (Quesada and Senar 2006). Here it was shown that all colour measurements stabilise and are comparable to measurements on live birds if measures are taken from more than 9 feathers. Measurements were done using a field portable spectrometer (Jaz; Ocean Optics), a xenon light source (Jaz-PX; Ocean Optics) and a bifurcated fibre-optic probe fitted with a cylindrical probe holder at the tip to standardize measuring distance $(8.0 \mathrm{~mm})$ and to exclude ambient light (Andersson and Prager 2006). We held the probe perpendicular to the surface of the feathers during the measurements. To imitate the plumage surface of the bird with the feather sample, we stacked the 12 feathers by superimposing four layers of three feathers on a black velvet surface (Quesada and Senar 2006). We calculated reflectance measures relative to a white tile using SPECTRASUITE v. 2.0.162. In total we took nine readings from the feathers, each of which in itself was an average of 12 scans, while rearranging the stack of feathers after every third measurement. Subsequently we calculated an overall mean reflectance spectrum for the avian visible spectrum (taken to be 320-700nm; Andersson and Prager, 2006). Using the TETRACOLORSPACE (Stoddard and Prum 2008) we calculated the photon catch of each colour cone type; ultraviolet sensitive or violet (UVS); short-wavelength-sensitive or blue (SWS); medium-wavelength sensitive or green (MWS) and long-wavelength-sensitive or red (LWS) (Stoddard \& Prum 2008). On the basis of these cone catches we calculated the SWS ratio, as a measure of intensity of the yellow plumage (Evans et al. 2010) using the following formula: 3${ }^{1}(U V S+M W S+L W S) / S W S$.

\section{Breast stripe size}

We took photos of all adult birds tested in the mate choice tests or caught during the breeding season. The camera (Casio EX-H30) was mounted on a portable copy stand maintaining a standard distance to the bird. While taking the photo of the breast stripe, we held the bird outstretched by its tarsi and beak on top of graph paper as a reference (Figuerola and Senar 2000). We defined breast stripe area as the area of the black band between the point of inflexion where the ventral stripe widens to a throat patch and the posterior end of the stripe. The area was calculated in square centimetres using image analysis software ImageJ (Abràmoff et al. 2004). 


\section{Body condition}

We calculated body condition as the residuals from a linear regression of mass and tarsus length. 



\section{Chapter 3}

Direct fitness benefits explain mate preference, but not choice, for similarity in heterozygosity levels

Lies Zandberg, Gerrit Gort, Kees van Oers, Camilla A. Hinde 


\begin{abstract}
Under sexual selection, mate preferences can evolve for traits advertising fitness benefits. Observed mating patterns (mate choice) are often assumed to represent preference, even though they result from the interaction between preference, sampling strategy and environmental factors. Correlating fitness with mate choice instead of preference will therefore lead to confounded conclusions about the role of preference in sexual selection. Here we show that direct fitness benefits underlie mate preferences for genetic characteristics in a unique experiment on wild great tits. In repeated mate preference tests, both sexes preferred mates that had similar heterozygosity levels to themselves, and not those with which they would optimise offspring heterozygosity. In a subsequent field experiment where we cross fostered offspring, foster parents with more similar heterozygosity levels had higher reproductive success, despite the absence of assortative mating patterns. These results support the idea that selection for preference persists despite constraints on mate choice.
\end{abstract}




\section{Introduction}

Thdividuals can incur fitness benefits by choosing the right mate (Andersson 1994). Therefore, preferences are expected to evolve for traits that advertise mate quality. Such preferences can be additive, or non-additive suggesting preference for compatibility (Neff and Pitcher 2005). Selection for compatible mates predicts that individuals prefer a mate with whom they would achieve the highest reproductive success, even though this mate may not necessarily be the universally 'best individual' (Drickamer et al. 2003; Ihle et al. 2015). Preference functions can have different directions and shapes (Edward 2015) and can consistently differ between individuals (Brooks and Endler 2001; Forstmeier and Birkhead 2004). Only by studying the reproductive benefits individuals gain from finding a preferred mate, we can unravel the selective pressures underlying the evolution of these mate preferences (Andersson 1994).

Even though mate preferences may be at the basis of mate choice, sampling strategy and environmental or social constraints can largely influence that choice (Jennions and Petrie 1997; Wagner 1998). For instance, the presence of intra-sexual competition and the availability of potential mates limit the possibilities for an individual (Jennions and Petrie 1997; Wagner 1998; Bro-Jørgensen 2002). Additionally, since searching for a mate is costly, the effort invested in finding a suitable mate may be dependent on the condition of the chooser (reviewed in Cotton et al. 2006b). Therefore, the mate that an individual gets (mate choice) may not represent mate preference, as often assumed. Correlating fitness benefits with the observed choice of social partner instead of the measured preferences will therefore lead to confounded conclusions about the role of mate preferences in sexual selection. Instead, to understand fitness consequences and the evolution of mate preferences, preference and choice should be studied as two distinct processes. Although studies on mate preference, mate choice and mating patterns, and on the fitness effects of choice are abundant, there is, to our knowledge, none that has combined all three and tested what the fitness benefits of mate preferences are under mate choice constraints in a wild population.

Genetic characteristics are important for reproductive success (Foerster et al. 2003; Kempenaers 2007; García-navas et al. 2009), and are thus expected to affect mate preference. Heterozygosity, the genetic variability within an individual, is known to be positively correlated with fitness aspects such as reproductive success, survival, immunocompetence and parasite resistance (reviewed in Kempenaers 2007 and Chapman et al. 2009). An individual can thus potentially increase its fitness by selecting a mate with which it would produce heterozygous and therefore 'fitter' offspring (Tregenza and Wedell 2000; Tomiuk et al. 2007; Szulkin et al. 2009). By finding an unrelated, or genetically 
dissimilar mate, offspring heterozygosity can be increased and the negative effects of inbreeding avoided when inbreeding depression is larger than outbreeding depression (Van de Casteele et al. 2003; Szulkin et al. 2007). Moreover, although most studies focus on these indirect effects, genetic traits can have direct effects on reproductive success as well, especially in species with biparental care. For instance, heterozygous females have been shown to lay larger clutches (Foerster et al. 2003), and heterozygous males have better territories (Seddon et al. 2004; Ryder et al. 2010), and feed their offspring more often (García-navas et al. 2009).

Here we tested 139 wild great tits, both males and females, for their preference for mate heterozygosity, relatedness and simulated offspring heterozygosity, and how such preferences relate to the choosers' own genetic characteristics. In a subsequent experiment, we studied how mating patterns relate to genetic characteristics. By cross fostering chicks between broods, we assessed the direct and indirect benefits of the (biological and foster) pairs' heterozygosity and relatedness on reproductive success. We aimed at investigating how mate preferences are reflected in mate choice (pair formation in the wild), and what the direct and indirect fitness benefits of these preferences are. Using this unique experiment, we were able to study the evolutionary benefits of sexual selection, by measuring mate preferences, and the reproductive benefits of these preferences under mate choice constraints in a wild population. Here we show that, despite the fact that mate preferences might not be reflected in choice, there is potential for selection on preferences for genetic advantages via direct reproductive benefits. 


\section{Methods}

\section{Study population}

We used wild great tits originating from six field sites for the preference experiments. We caught all focal birds (choosers in the preference test) in the main field site Boslust $\left(5^{\circ} 85^{\prime} \mathrm{E}\right.$, $52^{\circ} 01^{\prime} \mathrm{N}$ ), while the stimulus birds (chosen individuals in the preference tests) originated from more distant field sites Heijkamp $\left(5^{\circ} 83^{\prime} \mathrm{E}, 52^{\circ} 03^{\prime} \mathrm{N}\right)$, Roekelse bos $\left(5^{\circ} 71^{\prime} \mathrm{E}, 52^{\circ} 08^{\prime} \mathrm{N}\right)$, Westerheide $\left(5^{\circ} 84^{\prime} \mathrm{E}, 52^{\circ} 02^{\prime} \mathrm{N}\right)$, Lichtenbeek $\left(5^{\circ} 85^{\prime} \mathrm{E}, 52^{\circ} 00^{\prime} \mathrm{N}\right)$ and Bennekomse bos $\left(5^{\circ} 69^{\prime} \mathrm{E}\right.$, $52^{\circ} 00^{\prime} \mathrm{N}$ ) to minimize the chance they were familiar with the focal birds. Great tits pair throughout the winter period (Culina 2014), therefore we have tested birds in the months of January, February and early March, a period where they are highly likely to show mate preferences. On 8 evenings in total, between January - March in 2014 and 2015 we caught 420 birds in total while they were roosting in nest boxes (on two evenings in January 2014 we caught 30 birds and we caught 60 birds on 2 evenings in February - March 2014 and on 4 evenings in January - March 2015), and transported them to the bird housing facilities at the Netherlands Institute of Ecology (NIOO-KNAW, Wageningen, the Netherlands). At this facility, we banded and weighed them and photographed their breast stripe, after which we housed them individually in standard cages $(0.9 \times 0.4 \times 0.5 \mathrm{~m})$, with a solid bottom, top, side and rear walls, a wire-mesh front and three perches. The birds had ad libitum access to water and sunflower seeds, supplemented daily with commercial egg mixture, ground peanuts, live mealworms, soldier fly larvae, dead wax moth larvae, green bottle fly larvae and crickets. Following the 6 days of preference tests, we took a blood sample $(10 \mu \mathrm{l})$ by puncturing the brachial vein, and measured weight and tarsus length. After this we released the birds back into the field site of origin. Three birds out of the 420 birds caught died after their first night at the facilities of the NIOO-KNAW.

\section{Preference tests}

We carried out mate preference tests at NIOO-KNAW. All tests took place in a test room $(4.0 \times 2.4 \times 2.5 \mathrm{~m})$ with white walls, and high-frequency fluorescent lights to mimic natural lighting conditions. We tested individual mate preferences using a carrousel shaped sixchoice chamber (Figure 3.1). With the six-choice test we were able to measure individual directional and quadratic (stabilising or disruptive) preferences (Edward 2015). Great tits keep local dominance in their breeding territories throughout the winter, and often forage in larger fission-fusion foraging flocks. Encountering 6 birds of the opposite sex is therefore not unrealistic for this species. For every 10 focal birds we caught 20 stimulus birds (thus in total we always caught either 30 or 60 birds per evening). 18 of these birds participated in the experiment and two extra birds were caught in case of health or other issues. Each focal bird was tested with 3 different groups of 6 stimulus birds each. In our 


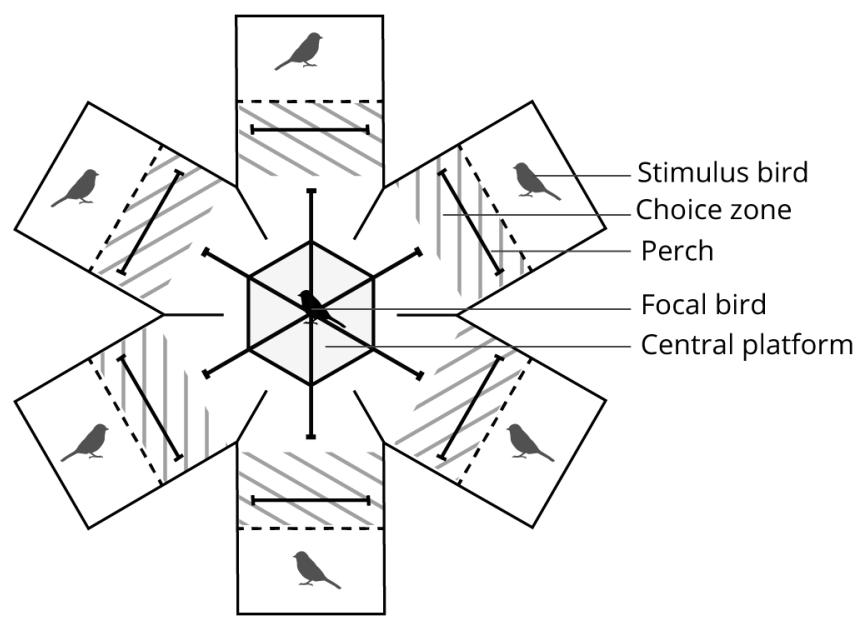

Figure 3.1- Experimental setup mate preference test. Birds were tested for their preference in a six-choice test room. From the hexagonal central platform the focal bird could observe all stimulus birds, while from the perches in the choice zone only one stimulus bird was visible. Time spent in each of the choice zones was measured.

final dataset we tested 69 focal females and 70 focal males for their preference with a total of 42 groups of 6 stimulus birds $(\mathrm{N}=252)$.

In this six-choice setup each focal bird could see all stimulus birds from the central perch, but when in front of a stimulus cage it could not see the other stimulus birds. The stimulus birds could not see each other (Figure 3.1). For more details on the setup see Appendix S1A in the Supporting information.

For every test, we placed six stimulus birds in the test setup and gave them 15 minutes to habituate. During the test, all birds had access to a piece of apple and before each test they received 5 mealworms in a cup (to hide them from view for the focal bird). In most cases, the stimulus birds had eaten all the mealworms before the focal bird was introduced. When the focal bird was introduced into the setup, recording started and lasted for 45 minutes. We tested each focal bird with 3 groups of different stimulus birds (groups A, B and $C$ ), of which one group was repeated (order of testing: ABCA). With this repeated test we tested the overall repeatability of preference for focal-stimulus combinations by conducting a one-way ANOVA with focal-stimulus combination as a factor and fraction association time as the dependent variable. Repeatability was calculated using the mean squares within and between focal-stimulus combinations $(r=0.20 \pm 0.039)$. For further analysis we only used the second repeat test on $\mathrm{A}$ in order to have equal numbers of tests for every focal-stimulus dyad and avoid habituation effects of the first test. Every stimulus bird participated in 13 or 14 trials with 10 focal birds, divided over 4 mornings and afternoons over a period of six days. We randomised the positions of the stimulus birds within group. Every group of stimulus birds was composed blind with regard to the natural variation in heterozygosity and relatedness to the focal bird. From the videos, we 
calculated the time spent in front of each stimulus bird, which is commonly used as a measure of preference. Great tits do not show clear courtship or copulation solicitation behaviours outside the fertile period and it is therefore difficult to validate these tests and the association time measure using behavioural data. However in numerous species association time in a choice test has been shown to predict courtship behaviours or pair formation (Clayton 1990; Witte 2006; Lehtonen and Lindström 2008; Jeswiet and Godin 2011) and to correlate with reproductive success (Drickamer et al., 2003). For our analysis, we modelled the proportion of association time, i.e. the association time with each stimulus bird divided by the total association time that this bird spent with all stimulus birds. Using this measure we were able to separate the directional preference for traits from variation in the motivation to choose (the total time spent in all choice zones) (Cotton, Rogers, et al. 2006). Trials in which the focal bird did not visit any of the stimulus birds (N $=53$ ) were excluded from the analysis.

\section{Reproductive success}

During the breeding seasons following the mate preference tests, we monitored all breeding attempts in the Boslust population, from where the focal individuals originated. Boslust is a mixed wood forest of approximately 70 ha containing 130 nest boxes. During the breeding season we checked unoccupied nest boxes every five days for nesting activity. For every nest we estimated the egg-laying date, start of incubation, hatching date and fledging date and recorded the clutch size, brood size, and the number of fledged offspring (as in Hinde 2006). We weighed the chicks on the day of hatching (day 0), and gave them a down code by selectively removing down feathers to individually identify each chick within a brood. These down codes were visible until at least day 6, after which we gave them a uniquely numbered aluminium ring. On day 1 after hatching we cross fostered the chicks with chicks from two other broods that had hatched on the same day, matched for weight as in Brinkhof et al (1999) in such a way that all chicks were raised by foster parents. In 2014 and 2015 we cross fostered 26 and 48 broods, with 191 and 332 chicks respectively. On day 3 after hatching we took a blood sample $(3 \mu \mathrm{l})$ from all chicks from the metatarsal vein. Chicks were weighed 14 days after hatching (to the nearest $0.01 \mathrm{~g}$ using a digital scale) as a measure of fledging weight, which is closely correlated with first year survival (Van Balen 1973), and we measured their tarsus length (to the nearest $0.1 \mathrm{~mm}$ using callipers). When the nestlings were 10 days old we caught the adults at the nest using spring traps. We recorded adult body mass, tarsus length and took a blood sample $(10 \mu \mathrm{l})$ from the brachial vein. We fitted unbanded adults with an aluminium numbered ring. Each nest was checked after 21-25 days for chick-fledging. 


\section{Genotyping and genetic traits}

We genotyped all birds that participated in the mate preference experiments (344 individuals) and the breeding season (142 adults, 486 chicks) across 20 polymorphic microsatellite markers. Using known mother-offspring dyads we detected the occurrence of null alleles and other irregularities. On the basis of this analysis we excluded three microsatellite loci from further analyses because of the non-reliability of their results. Full details on marker-characteristics and the genetic analyses can be found in Appendix S1-B. We used homozygosity by locus (Aparicio et al. 2006) to estimate individual genetic diversity and calculated this measure for all genotyped individuals with the $R$ package GENHET (Coulon 2010). Because the HL index represents homozygosity instead of heterozygosity we transformed the HL values into an estimate of heterozygosity by calculating the complement of HL (1-HL). Using the package Rhh (Alho et al. 2010) we determined that our sample of 17 microsatellites is very likely to be representative of genome wide heterozygosity. See Appendix S1-B for more details on the test of genome wide heterozygosity and the choice for HL as a measure of heterozygosity. For each focalstimulus dyad in the mate preference tests, we simulated offspring heterozygosity using the program STORM (Frasier 2008). For this the average HL estimate of 100 simulated offspring was calculated over 1000 iterations and averaged for each dyad ( $\mathrm{N}=2046)$.

We estimated marker-based relatedness by calculating the pairwise $r$ following the method of Wang (2002) in the program Coancestry (Wang 2011), as this measure fitted our social pedigree best (see Appendix S1-B). The relatedness values range from -1 to 1 , in which values of 0 represent random allele sharing, and positive and negative values respectively represent more and less sharing than at random, based on the allele frequencies in the population.

Using the microsatellite data from 17 loci, we assigned paternity of offspring using Cervus 3.07 (Marshall et al. 1998). For 49 offspring, in 23 broods, the social father was unlikely to be the sire. By comparing these offspring with all other males, we were able to identify the extra-pair father for 15 offspring (see Appendix S1-B).

\section{Statistical analyses}

\section{Mate preference}

To analyse the proportion of time spent associating with each stimulus bird we used a binomial generalized linear mixed model with a logit link function. The fixed part of the model contained as explanatory variables: heterozygosity of focal and of stimulus birds; relatedness, squared relatedness and offspring heterozygosity for each focal and stimulus dyad; sex of the focal bird; interaction of focal and stimulus heterozygosities, of focal bird's 
heterozygosity and relatedness, and of sex and aforementioned variables. The random part of the model contained different components, following the experimental set-up as closely as possible. In case of a significant interaction of continuous variables, we used as a check on possible shortcomings of the systematic part of the model categorized versions of the variables: we grouped the values of a variable into three groups, with cutpoints based upon tertiles, and formulated the model using these factors, thereby allowing a more flexible response surface. These extra analyses confirm the general patterns that we found in the interactions of the continuous variables, we therefore only report these in the supplementary information. For details on the analysis and results we refer to Appendix S1-C and S3-D respectively.

We started the analysis with an omnibus test on the full model. Since this was significant $(\mathrm{F}=1.95, \mathrm{P}=0.03)$ we proceeded with stepwise backward selection to obtain the minimal adequate models. Non-significant terms were deleted stepwise, starting with the highest order interactions and/or the least significant term $(\mathrm{P}<0.05)$. Because of the high number of variables in the model and the possibility of false positives we treated $P$ values between 0.05 - 0.01 with more caution and refrain from drawing strong conclusions. Because of this we will describe these results in more detail in Appendix S4. The full model containing all variables of interest is included in Appendix S5.

\section{Assortative mating}

We tested the null hypothesis of random pairing between males and females by performing a permutation test in which we compared the test statistic, that is, the correlation between the males and females of a pair, to a sampling distribution generated by randomly permuting the females and computing the correlation between randomly paired couples (10000 permutations).

We explored whether individuals paired differently from random with regard to relatedness. To test this we compared the observed distribution of relatedness between mates, with a distribution obtained if individuals mated randomly with regard to relatedness in $R$ (version 2.3.2, R Development Core Team 2015). We generated randomly mated pairs (within each year) with 100000 iterations and compared the confidence interval of the average relatedness between these randomly generated pairs with the average relatedness values in the observed population. The birds could have been restricted in their choice due to the local availability of potential mates; hence, we also ran an analysis accounting for spatial structure (see Appendix S3-B). Furthermore, to check whether catching birds for preference testing might have influenced mate choice patterns we also compared mating analyses between tested and untested pairs (see Appendix S3- 
C). These additional analyses did not change our results and therefore we present only the uncorrected analyses.

\section{Reproductive success}

We tested the effects of parent heterozygosity and relatedness on fledging weight (weight of the chicks when 14 days old), of both biological and foster parents. For this we ran a linear mixed model using the R package lme4 version (version 1.1-12, Bates et al. 2015), with biological brood and foster brood as random effects to account for the cross-foster design and the multiple chicks per brood. As explanatory variables we added heterozygosity of both parents of the biological and the foster parent pairs and the relatedness and square of relatedness between the pair members. For effects of biological fathers and relatedness we used the genetic sire, which in some cases was the extra-pair sire. We also tested for an interaction effect of parental heterozygosity on reproductive success. Additionally, to test whether the effect of the relatedness of the partner depended on the heterozygosity of the individual, we also tested the interaction between relatedness and heterozygosity for both biological and foster parents. Moreover, we also tested whether chick heterozygosity influenced weight by adding this as an explanatory variable to the model. Because brood size and offspring sex (as categorical variable) are known to affect chick weight we also controlled for these. We also added brood size, catch date, year (as a categorical variable) and the interaction between catch date and year (catch date * year) as control variables. we started the analysis with an omnibus test on the full model. Since this was found to be significant $\left(X_{20}^{2}=43.55, \mathrm{P}=0.002\right)$ we proceeded with stepwise backward selection to obtain the minimal adequate models.

We tested for differences in fledging probability for each chick with a binary generalized linear mixed model using the $R$ package $l m e 4$ (Bates et al. 2015), with fledging (yes or no) as a dependent variable and heterozygosity, relatedness for biological and foster parents as explanatory variables. We also tested for an interaction effect of parental heterozygosity. To test whether relatedness effects differed depending on the heterozygosity of the parents we included the interaction between relatedness and heterozygosity for biological and foster parents. We added brood size, hatch date, offspring sex and year as control variables (offspring sex and year as a categorical variables). Biological brood and foster brood were random effects to account for the crossfoster design. Similar to the preference models we started the analysis with an omnibus test on the full model. Since this found to be significant $\left(X_{13}^{2}=29.34, \mathrm{P}=0.006\right)$ we proceeded with stepwise backward selection to obtain the minimal adequate models. In case of significant interactions, we checked the systematic part of the model using categorized versions of the variables (see Appendix S3-D) and we treated P values between 0.05 - 0.01 
with more caution. These results are described in more detail in Appendix S4. Full models containing all variables of interest are included in Appendix S5. 


\section{Results}

\section{Mate preferences}

Individuals did not spend more time with stimulus birds with which they could theoretically optimise offspring heterozygosity (HLoffspring, GLMM: $\mathrm{F}_{1,66.3}=-0.24, \mathrm{P}=0.81$, Table 1). Time spent with a stimulus bird was influenced by an interaction between the chooser's and stimulus birds' heterozygosity (Figure 3.; HLfocal * HLstimulus, GLMM: $\mathrm{F}_{1,62.93}=2.76, \mathrm{P}=0.008$, Table 1). Very heterozygous birds spent more time with heterozygous birds, and vice versa, homozygous birds spent more time with homozygous birds.

\section{Assortative mating}

Birds did not mate assortatively for heterozygosity. There was no difference between heterozygosity correlations within pairs and a randomly generated distribution of pairs drawn from the population (random correlation 95\% confidence interval $=\left[\begin{array}{ll}-0.23,0.25\end{array}\right]$; correlation breeding pairs $=-0.07 ; \mathrm{N}=70$ breeding pairs; 10000 permutations).

Individuals did not mate differently from random with regard to relatedness. The randomization test showed no difference between relatedness within the breeding pairs and randomly generated pairs drawn from the population (random relatedness $95 \%$ confidence interval = [$0.026,0.022]$; average relatedness breeding pairs $=0.009 ; \mathrm{N}=70$ breeding pairs; 100000 iterations). For the complete observed and simulated distribution of relatedness and heterozygosity see Appendix S3-A.

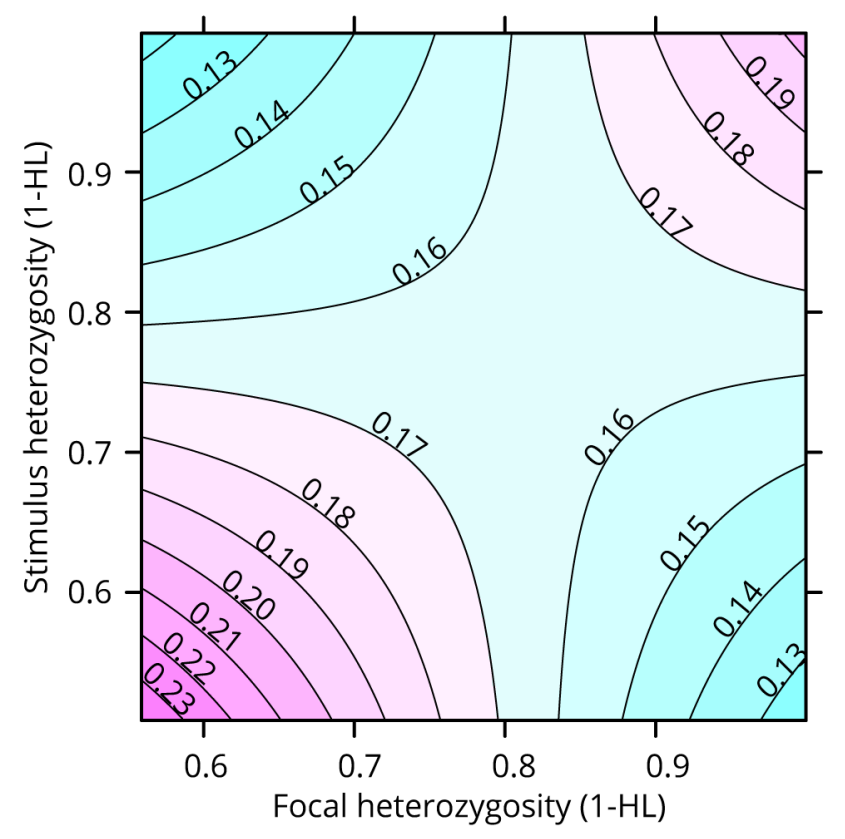

Figure 3.2-Preferences for genetic characteristics. Individuals spent more time with stimulus birds with similar heterozygosity levels. Association time with each stimulus bird was calculated as the fraction of the total time spent with all stimulus birds. Red colours indicate that relatively more time was spent and green colours indicate that relatively less time was spent. 
Table 1 - Mate preferences - minimal adequate model. Table consists of all factors tested in the binomial mixed model with proportion of time spent with each of the stimulus birds as the dependent variable ( $N$ focals=116, $N$ tests=359). Given is the estimate, the degrees of freedom (df), the test statistic ( $F$ value) and the significance ( $P$ value). A random effect for stimulus bird identity (mean $\pm S E ; 0.22 \pm 0.04$ ) $r$ and random slopes for focal bird identity with respect to the stimulus bird's heterozygosity $(2.41 \pm 0.70)$, relatedness $(1.46$ \pm 0.47 ), and offspring heterozygosity $(3.23 \pm 1.98)$, and a random effect for test number (to allow for negative correlations among association times within one six-choice test; $(-13.89 \pm 0.52)$ and an extra scale parameter on the original scale, were included in the model. Using backwards elimination of factors, the P values, $d f$ and test statistics given come from the last model in which the factor or interaction was included. Degrees of freedom for $F$ - and $t$-tests were calculated using the degree of freedom approximation proposed by Kenward and Roger (1997).

\begin{tabular}{lccccc}
\hline & Estimate & Num df & Denom df & F value & P value \\
\hline Minimal adequate model: & -1.63 & 1 & 66.46 & - & - \\
Intercept & 0.14 & 1 & 204.90 & 2.47 & 0.01 \\
HLfocal & 0.03 & 1 & 160.20 & 0.05 & 0.96 \\
HLstimulus & 6.76 & 1 & 62.93 & 2.76 & $\mathbf{0 . 0 0 8}$ \\
HLfocal $^{*}$ HLstimulus & 0.30 & 1 & 63.05 & 1.65 & 0.10 \\
Relatedness & -2.33 & 1 & 274.20 & -2.39 & 0.02 \\
Relatedness & -3.10 & 1 & 277.10 & -2.06 & 0.04 \\
Relatedness * HLstimulus & & & & & \\
\hline Dropped terms: & -0.13 & 1 & 66.30 & -0.24 & 0.81 \\
HLoffspring & 0.02 & 1 & 61.65 & 0.23 & 0.82 \\
Sex (female) & 0.95 & 1 & 164.80 & 0.93 & 0.35 \\
HLstimulus * sex (female) & 5.67 & 1 & 61.06 & 1.05 & 0.30 \\
HLfocal * HLstimulus * sex (female) & 0.90 & 1 & 43.50 & 0.94 & 0.35 \\
HLoffspring $^{*}$ sex (female) & -1.42 & 1 & 63.37 & -0.66 & 0.51 \\
Relatedness * HLfocal & -0.29 & 1 & 62.97 & -0.68 & 0.50 \\
Relatedness * sex (female) & 1.55 & 1 & 282.00 & 0.71 & 0.48 \\
Relatedness * sex (female) & & & & & \\
\hline
\end{tabular}

\section{Reproductive success}

The relatedness of foster parents showed a quadratic relationship with fledging weight (on day 14); very unrelated and very related foster parents reared heavier offspring (Figure 3.; relatedness ${ }^{2}$, LMM: $\mathrm{t}_{34.91}=3.19, \mathrm{P}=0.003$, table $\mathrm{S} 2$ ). 
Heterozygous offspring were not more likely to fledge than homozygous offspring (HLoffspring, GLMM: $\mathrm{Z}=0.28, \mathrm{P}=0.78$, Table 2). However, offspring from foster parents with similar levels of heterozygosity had a higher chance of fledging than offspring from dissimilar foster parents (Figure 3.4a; HLfoster female * HLfoster male, GLMM: Z=3.26, $P<0.001$, Table 2). Biological parents produce offspring with higher fledging success when they are more related and the female is more homozygous (Figure 3.4b; HLbiological female * biological relatedness, GLMM: $Z=-3.31$, $\mathrm{P}<0.001$, Table 2).

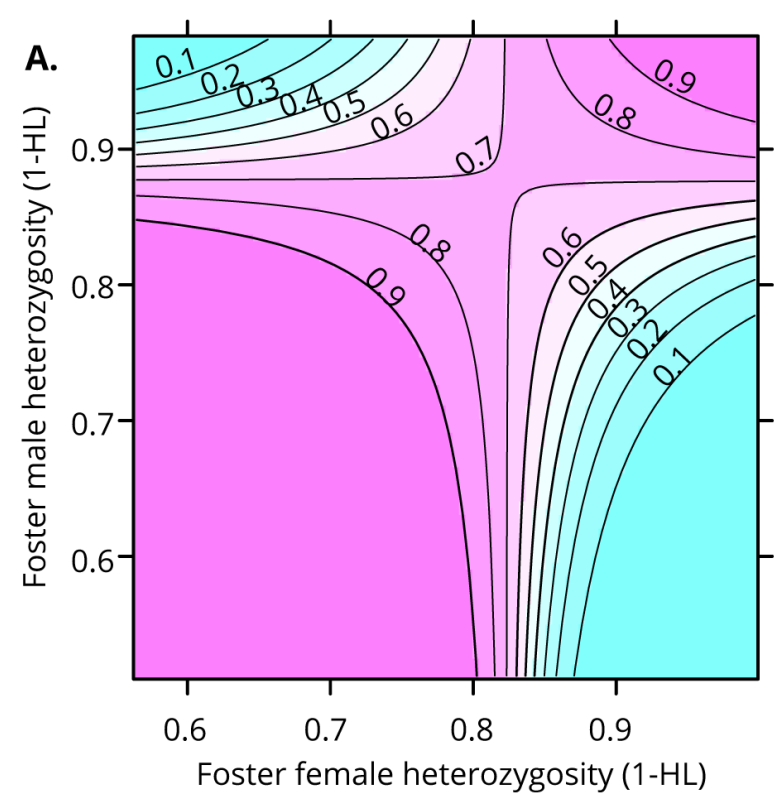

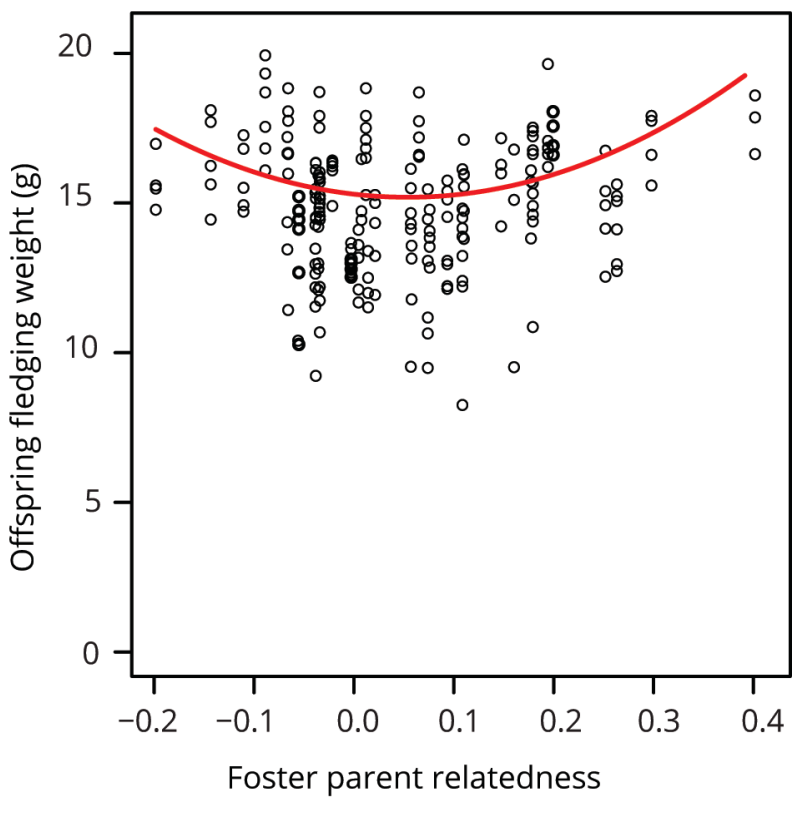

Figure 3.3 - Effects of genetic characteristics on offspring fledging weight. Offspring from very related or very unrelated foster parents had a higher fledging weight than offspring from moderately related parents.

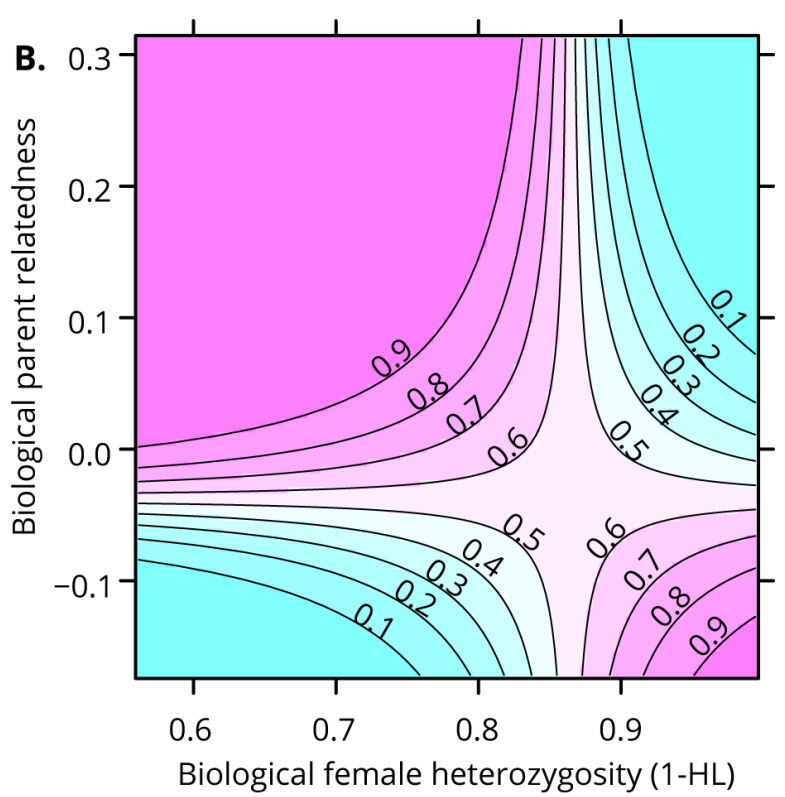

Figure 3.4-Effects of genetic characteristics on fledging success of chicks. A) Offspring from foster parents with similar heterozygosity levels had a higher chance of fledging. B) Fledging probability depended on the heterozygosity of the biological mother and the biological parents' relatedness. Red colours indicate a higher fledging probability and green colours indicate a lower fledging probability. 
Table 2. Offspring fledging probability - minimal adequate model. Table consists of all factors tested in the binary mixed model with the fledging probability of the offspring (0/1) as the dependent variable $(N=272)$. Random effects for biological brood (var $\pm s d: 0.00 \pm 0.00)$ and foster brood $(3.63 \pm 1.90)$ were included in the model. Given is the estimate, the degrees of freedom (df), the test statistic ( $Z$ value) and the significance (P value). Biological and foster brood identity were included as random factors. Using backwards elimination of factors, the P-values, df and test statistics given come from the last model in which the factor or interaction was included.

\begin{tabular}{lccc}
\hline & Estimate & Z value & P value \\
\hline Minimal adequate model: & & & \\
Intercept & 0.45 & - & - \\
HLfoster female & -10.02 & -1.96 & 0.05 \\
HLfoster male & 3.44 & 0.83 & 0.40 \\
HLfoster female * HLfoster male & 176.96 & 3.26 & $<\mathbf{0 . 0 0 1}$ \\
HLbiological female & -6.28 & -1.51 & 0.13 \\
HLbiological male & -5.15 & -2.07 & 0.04 \\
Biological relatedness & 5.39 & 1.89 & 0.06 \\
HLbiological female * biological relatedness & -169.41 & -3.31 & $<\mathbf{0 . 0 0 1}$ \\
Offspring sex (male) & -0.33 & -0.86 & 0.39 \\
Brood size & -0.30 & -1.03 & 0.31 \\
Hatch date & 0.21 & 1.27 & 0.20 \\
Year (2015) & -0.01 & -0.01 & 0.99 \\
\hline Dropped terms: & & & \\
HLoffspring & 0.66 & 0.28 & 0.78 \\
Foster relatedness & 5.48 & 1.46 & 0.14 \\
HLfoster female * Foster relatedness & 33.95 & 0.78 & 0.43 \\
HLfoster male ${ }^{*}$ Foster relatedness & 16.83 & 0.37 & 0.71 \\
HLbiological female * HLbiological male & -3.08 & -0.08 & 0.93 \\
HLbiological male * biological relatedness & -19.18 & -0.70 & 0.48 \\
\hline
\end{tabular}




\section{Discussion}

Despite great interest in the evolution of mating preferences and numerous studies of sexual selection processes, few actually test how selection acts on mating preferences in natural populations. Here we show that both males and females preferred potential mates with similar levels of heterozygosity to their own. While we did not find these mate preferences reflected in the observed mating patterns, individuals gained direct fitness benefits (greater fledging success) from mating assortatively for heterozygosity. These direct benefits indicate the potential for selection on preferences for genetic benefits.

Against our expectations, we did not find any mating patterns with regard to heterozygosity or relatedness. This suggests that, despite their preferences, great tits, in this study, do not all obtain a partner according to their preference. Mating patterns can differ from preferences due to different factors (Wagner 1998). The great tit is a territorial species with biparental care. Therefore, individuals become unavailable after mating and the number of available mates at any time is limited. As they have to choose from what is available at that place and time, their choice may be limited and they might have to settle with a less preferred partner. Despite this, they may still choose according to their preference from this limited pool of available mates. Moreover, compared to the sample sizes in the mate preference tests, we have only a limited dataset on mate choice. Possibly, because of these two reasons, we were not able to pick up on mating patterns in the data. Moreover, we also checked if mating patterns of birds used in the preference tests could have been affected by catching them for these tests, however, this did not seem to be the case (see Appendix S3). The difference between preference and choice that we found here illustrates the importance of considering them as two distinct processes, both of which should be studied independently. By measuring preferences and the fitness aspects correlated with it, it is possible to show how selection works on these preferences (Jennions and Petrie 1997). We strongly advise that the terminology for mate preference (functions) and mate choice should be used consistently, to be able to compare studies and draw conclusions (Jennions and Petrie 1997; Wagner 1998; Edward 2015).

We expected individuals to prefer heterozygous partners, as heterozygous parents may produce more heterozygous offspring (Mitton et al. 1993; Hoffman et al. 2007), and have been shown to invest more in their offspring (Foerster et al. 2003; García-navas et al. 2009). In our preference experiments, however, we found that rather than having a uniform preference for the most heterozygous mate (Kempenaers 2007), individuals showed a strong preference to spend time with individuals that had similar heterozygosity levels to themselves. A number of studies have found similar assortative mating patterns for heterozygosity (Bonneaud et al. 2006; García-navas et al. 2009). Under the assumption that 
all individuals have the same preference for heterozygous mates, the authors suggest that assortative mating is due to competition over mates. In our preference experiments however, this pattern represents the actual preference, not choice, for similarity in heterozygosity. This preference is likely to represent a behavioural, rather than a genetic compatibility, since it benefitted the chicks through an effect of foster parents. One potential explanation for this surprising pattern may be that, assuming that heterozygosity is a trait indicating quality, assortative preferences have evolved to save low quality individuals energy and time in searching and to lower the risks of being abandoned by their mate for a higher quality individual (Burley 1988; Johnstone 1997; Mcnamara et al. 1999; Holveck and Riebel 2010). Alternatively, individuals with similar heterozygosity may have a higher behavioural compatibility resulting in direct benefits of having this preference.

The consequences for this preference were reflected in fledging success, as chicks raised by pairs with similar heterozygosity had a higher chance of fledging. This effect was not due to pre-hatching effects such as genetic and early maternal effects, but a post-hatching rearing effect from the foster parents. From our data we cannot determine through which mechanism these assortative pairs were more successful in rearing offspring. However, similar to our results, offspring mortality during the rearing period in zebra finches depended on the compatibility of the foster parents (Ihle et al. 2015). Possibly individuals invested more when they were able to obtain a mate they preferred, regardless of its apparent heterozygosity or quality (Drickamer et al. 2003; Ihle et al. 2015).

Against our expectations, we did not find individuals to prefer a mate with which they would produce more heterozygous offspring, which have a higher expected fitness prospect (Kempenaers 2007). Moreover, during the breeding season, we did not observe any pairing patterns based on a choice for relatedness levels. Although inbreeding has been shown to have negative effects on fitness in great tits (Szulkin et al. 2007), no preference or avoidance of inbreeding in mating patterns was observed in the same population (Szulkin et al. 2009), which is similar to the patterns we found in our population. Nevertheless, we did find a direct effect of relatedness, which is not associated with optimal in- or outbreeding, but an effect of the relatedness levels of the foster parents that the chicks are reared by. The offspring raised by moderately related parents are lighter, suggesting lower provisioning efforts and reproductive investment than in very related or very unrelated pairs. Which exact mechanism could underlie this higher reproductive investment for very related and unrelated pairs cannot be concluded from this dataset. Possibly the higher investment in these cases occurs because of different causes; when the pair is very related there are inclusive fitness benefits to be gained (Wang 
2011) and when the pair is unrelated, and therefore genetically compatible, they invest more in potentially higher quality offspring (Burley 1988; Mays and Hill 2004).

Additionally, the effect of relatedness on fledging probability depended on the female's heterozygosity levels. Although we hypothesised that especially homozygous individuals would obtain genetic benefits from finding an unrelated partner to increase their offspring heterozygosity, when biological mothers were homozygous, chicks had a higher fledging probability when she mated with a related male. These effects of the biological parents were surprising, especially since we expected the genetic characteristics to have a positive effect on offspring performance through offspring heterozygosity. Possibly these fitness effects of heterozygosity will only become apparent later in life (Szulkin et al. 2007). Apart from genetic effects the higher fledging success might also work through other prehatching effects that may be related to male attractiveness, such as maternal investment in egg size (Horváthová et al. 2012), yolk carotenoids (Marri and Richner 2014) or yolk androgens (Kingma et al. 2009).

Our results highlight the importance of testing not just for the indirect but also for the direct benefits of choice for genetic traits. For species with biparental care, most studies correlate parental heterozygosity or relatedness with different aspects of reproductive success, such as growth, provisioning or fledging success. Often, when no direct benefits can be observed, genetic effects are assumed by default (Ryder et al. 2010). By using a cross-foster design these direct and indirect effects can be teased apart to elucidate why individuals show particular mate preferences and what the benefits are of these preferences.

To conclude, in contrast to what is commonly assumed, we found that individuals do not show mate preferences that optimise offspring heterozygosity, but individuals prefer moderately related individuals with similar heterozygosity levels. These preferences for heterozygosity similarity can be explained by direct fitness benefits, indicating the importance of considering both indirect and direct benefits and effects of behavioural compatibility when studying mate choice for genetic traits. Moreover, our findings highlight the importance of studying preference and choice as two distinct processes, to further understand the selection pressures working on preferences. In addition, despite the fact that in natural situations mating patterns are not always determined by preference alone, we indicate the potential for selection for preferences for genetic characteristics via reproductive benefits. 


\section{Acknowledgements}

We are grateful Maaike Griffioen, Maxime Kole, Lisanne van den Bos, Antoine Juigner, Kristin Sauerland, Neeltje Hermus, Coen van Tuijl and Peter Clark for their help with collecting the data analysed here. Many thanks to Piet de Goede, Marylou Aaldering, Ruben de Wit, Coretta Jongeling and Anouk de Plaa for their help with taking care of the birds, and to Christa Mateman for her advice and help with the genetic analyses. We also thank Stichting Geldersch Landschap en Kasteelen for permission to do fieldwork in the Boslust forest. Moreover, we thank Tom Tregenza for his valuable comments on an earlier version of this manuscript. We are grateful to Alexandre Courtiol and two anonymous referees for their helpful suggestions. This work was partly financed by a WIAS PhD scholarship from Wageningen University and Research and the Royal Netherlands Academy of Arts and Sciences. This research was supported by 'SEX SEL-VAR' project number 334544 funded by Marie Curie Career Integration Grant from the European Commission (FP7). Permission for animal experiments was granted by the Animal Ethics Committee of the KNAW (DEC-KNAW) under protocol number NIOO13.12 to KVO and LZ.

\section{Author contributions}

LZ, CAH and KVO designed the study, LZ collected the data, LZ and GG analysed the data and LZ drafted the initial version of the manuscript. All authors contributed to later versions of the manuscript. 
Supplement to chapter 3 


\section{Appendix S1: Methods}

\section{A. Detailed description of the mate choice setup}

The mate choice setup consisted of a central zone for the focal bird (diameter: $1.48 \mathrm{~m}$, height: $1.10 \mathrm{~m})$ and 6 stimulus cages placed around the central zone $(0.5 \times 0.3 \times 0.5 \mathrm{~m})$. The stimulus cages were separated from the central area by UV-transmitting transparent PMMA panels, to allow visual contact between the focal bird and a stimulus bird. All birds were within auditory range of each other. The ceiling of both the focal compartment and of the stimulus cages was also made of UV transmitting PMMA. In the middle of the central focal compartment we placed a hexagonal platform $(0.4 \mathrm{~m}$ high) to prevent visual contact between the stimulus birds. On top of this we placed a perch with 6 branches of equal length in the direction of the stimulus cages, from which the focal bird could observe all stimulus birds. Two perches were present in front of every stimulus cage for the focal bird to sit on in proximity of the stimulus bird. From this position it could only observe that specific stimulus bird. The movements of the focal bird were recorded using a central camera pointing down from the ceiling (Panasonic WV-CP500). Using EthoVision XT software (Noldus Information Technology) we analysed the videos to calculate for every test the time that the focal bird spent in each choice zone.

\section{B. Genetic analyses and microsatellite markers}

DNA was isolated from blood samples using the 96-well Genomic DNA Kit following the manufacturer's instruction (FAVORGEN Biotech Corporation, Taiwan) and was quantified using a Nanodrop (Thermo Fisher Scientific). We genotyped all birds that participated in the mate preference experiments (344 individuals), and the breeding season (142 adults, of which 86 had been in the mate choice experiment, and 426 chicks) across 20 polymorphic microsatellite markers. Blood samples of 9 birds tested in the mate preference tests were missing and thus omitted from the genotype analysis. In the breeding season 37 chicks died before a blood sample could be taken and were therefore not genotyped. Using known mother-offspring dyads it was possible to detect the occurrence of null alleles and other irregularities. On the basis of this analysis the following three microsatellite loci were excluded from further analyses because of the nonreliability of their results: PmaD130, PmaGAn40 and Pma196 (Kawano 2003; Saladin et al. 2003). See table $S 1$ for the properties of the markers used in this study. 
Table S1. Summary of properties of microsatellite markers used in this study. The table shows the following information: range of allele sizes, category (neutral or functional), number of alleles (K), expected heterozygosity ( $\left.H_{E x p}\right)$, observed heterozygosity (Hobs), deviations from Hardy-Weinberg Equilibrium (HW), with NS = not significant and ND = not done, and reference for each locus. Summary is based on samples from all samples from adult birds ( $N=486)$ analysed using CERVUS (Marshall et al. 1998).

\begin{tabular}{lcccccc}
\hline Locus & $\begin{array}{c}\text { Allele } \\
\text { sizes }\end{array}$ & $\mathbf{k}$ & Hexp & Hobs & HW & Reference \\
\hline PmaC25 & $310-340$ & 11 & 0.862 & 0.879 & NS & Saladin et al. 2003 \\
PmaD105 & $375-427$ & 14 & 0.835 & 0.841 & NS & Saladin et al. 2003 \\
PmaGAn27 & $196-266$ & 25 & 0.922 & 0.904 & NS & Saladin et al. 2003 \\
PmaTAGAn71 & $171-214$ & 12 & 0.808 & 0.812 & NS & Saladin et al. 2003 \\
PmaTGAn33 & $249-331$ & 21 & 0.897 & 0.901 & NS & Saladin et al. 2003 \\
PmaCAn1 & $112-143$ & 14 & 0.836 & 0.800 & NS & Saladin et al. 2003 \\
Pma303 & $148-153$ & 2 & 0.165 & 0.165 & ND & Kawano 2003 \\
PmaTGAn64 & $301-323$ & 7 & 0.390 & 0.400 & NS & Saladin \& Richner 2012 \\
PmaD22 & $386-456$ & 18 & 0.900 & 0.868 & NS & Saladin et al. 2003 \\
PmaTGAn59 & $85-144$ & 21 & 0.915 & 0.905 & NS & Saladin \& Richner 2012 \\
Titgata87 & $161-311$ & 39 & 0.891 & 0.837 & NS & Wang et al. 2005 \\
PmaTAGAn73 & $212-251$ & 11 & 0.811 & 0.834 & NS & Saladin \& Richner 2012 \\
PmaCAn2 & $99-160$ & 27 & 0.907 & 0.901 & NS & Saladin \& Richner 2012 \\
PmaTAGAn86 & $141-223$ & 23 & 0.866 & 0.821 & NS & Saladin et al. 2003 \\
Pma69u & $218-244$ & 11 & 0.723 & 0.705 & NS & Kawano 2003 \\
PmaTGAn54 & $345-452$ & 31 & 0.872 & 0.824 & NS & Saladin \& Richner 2012 \\
Titgata84 & $554-584$ & 9 & 0.549 & 0.564 & NS & Wang et al. 2005 \\
\hline
\end{tabular}

\section{Extrapair paternity}

Using the microsatellite data from 17 loci, paternity of chicks was assigned using a likelihood approach in the software program Cervus 3.07 (Marshall et al. 1998). These loci had a combined second-parent exclusion probability (Pre) of 0.9999999945 . We calculated critical values of LOD (log likelihood ratio) and delta (difference in LOD scores between the most likely candidate parent and the second most likely candidate parent) using the following parameters in CERVUS: 10000 cycles, $98 \%$ of loci typed, error rate $0.01 \%$, two candidate parents. Offspring were assigned to be extra-pair when these critical values were exceeded in the comparison of the genotypes of the mother, the putative father and the offspring. 49 offspring of in total 23 broods were classified to be sired by an extra-pair father. By comparing these offspring genotypes with all known males from this field site in our dataset, we were able to identify the extra-pair father for 15 offspring.

\section{Heterozygosity}

Using the R-package 'Inbreedr' (Stoffel et al. 2016) we tested whether our sample of 17 microsatellites could be used as a measure of genome-wide heterozygosity by calculating 
the heterozygosity-heterozygosity correlation ( $\mathrm{HHC}$ ) and the $\mathrm{g}_{2}$ estimator of identity disequilibrium (Balloux et al. 2004; David et al. 2007; Stoffel et al. 2016). For this analysis we used the genotypes from all adult birds ( $N=486)$ (Balloux et al. 2004). Adding chicks in this analysis would overestimate the presence of rare alleles and with it heterozygosity for these alleles, causing a lower heterozygosity-heterozygosity correlation. Correlating heterozygosity based on one half of the markers with the heterozygosity of the other half of the markers gave a mean correlation of $\mathrm{r}=0.08,95 \% \mathrm{CI}=0.004-0.148$ (1000 iterations). Moreover, the $\mathrm{g}_{2}$ (David et al. 2007) for this dataset differed significantly from zero g2 = $0.0019, \mathrm{P}=0.04$ (1000 iterations, and 1000 permutations). Thus, together, the HHC and g2 indicate that marker heterozygosity is representative of genome-wide heterozygosity in this study system.

As a measure for heterozygosity we calculated homozygosity by locus (HL; Aparicio et al. 2006). HL accounts for allele frequencies and rare alleles, which makes it particularly suitable for populations with high immigration rates, and a high expected heterozygosity (above 0.4-0.6) (Aparicio et al. 2006). As with this population and this set of alleles the expected heterozygosity lies around 0.77 , the HL as a measure for heterozygosity is very appropriate. We therefore decided to use HL as a measure of heterozygosity. With this measure, birds that are completely homozygous have values of 1 , and completely heterozygous individuals would have a value of 0 . Because the HL index represents homozygosity instead of heterozygosity we transformed the HL values into an estimate of heterozygosity by calculating the complement of HL (1-HL).

Birds used in the mate preference tests had heterozygosity levels of 0.52-1.00 with a mean of $0.81 \pm 0.005$ (mean \pm SEM (standard error of the mean) $(\mathrm{N}=344)$. In the breeding season birds had heterozygosity levels between $0.52-1.00$ with a mean of $0.83 \pm 0.01$ (adults: $\mathrm{N}=142$; chicks: $\mathrm{N}=426$ ).

\section{Relatedness}

We estimated marker-based relatedness by calculating the pairwise $r$ following the method of Wang (2002) in the program Coancestry (Wang 2011). By calculating $r$ for full sibling pairs (extra-pair chicks were excluded) using different methods we determined that, for this population and these microsatellite markers, the relatedness measure using the method of Wang (2002) best fitted our social pedigree (different methods of calculating pairwise $r$ for full siblings and its associated standard error of the mean: $0.46 \pm 0.004$ SEM, Queller and Goodnight, 1989; $0.42 \pm 0.07$, Lynch and Ritland, 1999; $0.47 \pm 0.004$ Li et al., 1993; $0.48 \pm 0.004$, Wang, 2002). The relatedness values range from -1 to 1 , in which values of 0 represent random allele sharing, and positive and negative values respectively represent more and less sharing than at random, based on the allele frequencies in the 
population. In the mate preference experiments the relatedness between the focal and stimulus birds, originating from different field sites, ranged between -0.31 and 0.405 and a mean of $-0.01 \pm 0.002(\mathrm{~N}=2046)$. In the breeding season pairs within the experimental area had a relatedness of between -0.17 and 0.32 with a mean of $0.02 \pm 0.01(\mathrm{~N}=70)$.

C. Statistical analysis mate preferences (As described in the main paper, with more detail added)

To analyse the proportion of time that a focal bird spent associating with each stimulus bird we used a binomial generalized linear mixed model (GLMM) with a logit link function. The fixed part of the model contained as explanatory variables heterozygosity of both the focal and the stimulus birds, relatedness between each focal and stimulus dyad, offspring heterozygosity for each focal and stimulus dyad and sex of the focal bird. We also added the square of relatedness since a preference for moderately related individuals can be expected (Bateson 1983). To test for differences in preference depending on the chooser's traits we added the interaction between heterozygosity of the focal and the stimulus bird, and the interaction between the focal heterozygosity and relatedness. To test for sex differences, we also included interaction effects between sex and the previously mentioned explanatory variables.

Modelling the combined effect of continuous explanatory variables like the focal bird's heterozygosity, stimulus bird's heterozygosity and their interaction is necessarily sparse: only three parameters are used to describe the combined effect. To check whether the systematic trend captured in this way is not too restrictive, we also modelled the effect of these variables after categorization of each into three groups, based upon tertiles. Replacing the two regressors and their product by the categorized versions and their interaction, leads to a model with eight parameters replacing the earlier three. This model is more flexible than the original one, although it has its own shortcomings (Altman 2005). For the random part of the GLMM we followed the experimental design as closely as possible, specifying the next random terms (on the logit scale): 1) random effects for stimulus birds, since each stimulus bird was tested repeatedly; 2 ) random slopes for focal birds with respect to the stimulus bird's heterozygosity, relatedness, and offspring heterozygosity, as each focal bird was tested multiple times. Together these random effects define the G-side covariance structure. Furthermore, for the R-side covariance structure we allowed the six proportions per test to be negatively correlated (as they sum to one per six-choice test), by introducing a compound symmetric correlation structure at the proportion scale and we introduced an extra scale parameter, because we analysed a continuous proportion, for which the binomial variance-mean relationship only holds up to a scale factor. The statistical analysis was performed using procedure PROC GLIMMIX 
from the SAS software system (version 9.3; SAS Institute Inc., Cary, NC). We fitted the model using backward elimination for the fixed part of the model, removing first higher order terms and later lower order terms if not significant $(\mathrm{P}>0.01)$. The reported $\mathrm{P}$ value for an explanatory variable is the $\mathrm{P}$ value in the last model in which it still occurred, or in the final model if not removed (see table 1). 


\section{Appendix S2: Offspring fledging weight minimal adequate model}

Table S2 Offspring fledging weight minimal adequate model All factors tested in the linear mixed model with the fledging weight $(\mathrm{mg})$ of the offspring as the dependent variable ( $N=205)$. Biological (var \pm sd: $1619 \pm$ 40.24) and foster brood identity $(15724 \pm 125.40)$ were included as random factors (residual variation $=25030 \pm$ 158.21). Given is the estimate, the degrees of freedom (df), the test statistic ( $t$ value) and the significance ( $P$ value). Using backwards elimination of factors, the P values, df and test statistics given come from the last model in which the factor or interaction was included. Degrees of freedom for $F$ - and $t$-tests were calculated using the degree of freedom approximation proposed by Kenward and Roger(1997).

\begin{tabular}{|c|c|c|c|c|}
\hline & Estimate & df & t value & $P$ value \\
\hline \multicolumn{5}{|l|}{ Minimal adequate model } \\
\hline Intercept & 1585.82 & 54.80 & - & - \\
\hline HLfoster male & 535.65 & 29.13 & 2.24 & 0.03 \\
\hline Foster relatedness & -379.03 & 33.17 & -1.18 & 0.25 \\
\hline Foster relatedness ${ }^{2}$ & 5655.18 & 34.91 & 3.19 & 0.003 \\
\hline Offspring sex (male) & -64.38 & 175.12 & -2.67 & 0.008 \\
\hline Brood size & 31.86 & 34.34 & 1.97 & 0.06 \\
\hline Catch date & 7.13 & 28.24 & 0.13 & 0.90 \\
\hline Year (2015) & -100.83 & 28.61 & -1.63 & 0.11 \\
\hline Year $(2015) *$ catch date & 34.59 & 28.65 & 0.55 & 0.59 \\
\hline \multicolumn{5}{|l|}{ Dropped terms } \\
\hline HLoffspring & 11.29 & 120.82 & 0.06 & 0.95 \\
\hline HLfoster female & -103.86 & 29.69 & -0.41 & 0.68 \\
\hline HLfoster female ${ }^{*}$ HLfoster male & 1892.40 & 27.27 & 0.89 & 0.38 \\
\hline HLfoster female ${ }^{*}$ foster relatedness & -2380.31 & 29.04 & -0.85 & 0.40 \\
\hline HLfoster male $*$ foster relatedness & 143.28 & 29.02 & 0.06 & 0.96 \\
\hline HLbiological female & -218.12 & 18.84 & -1.14 & 0.27 \\
\hline HLbiological male & -277.76 & 55.10 & -1.34 & 0.19 \\
\hline HLbiological female ${ }^{*}$ HLbiological male & -1342.72 & 35.57 & 0.46 & 0.65 \\
\hline Biological relatedness & 251.52 & 36.48 & 1.25 & 0.22 \\
\hline Biological relatedness ${ }^{2}$ & -683.30 & 1274.16 & 0.54 & 0.60 \\
\hline HLbiological female * biological relatedness & -2575.63 & 137.95 & -1.40 & 0.16 \\
\hline HLbiological male * biological relatedness & 1181.14 & 39.36 & -0.50 & 0.61 \\
\hline
\end{tabular}




\section{Appendix S3: Additional analyses}

A. Observed and simulated distribution of relatedness and heterozygosity in pairs.
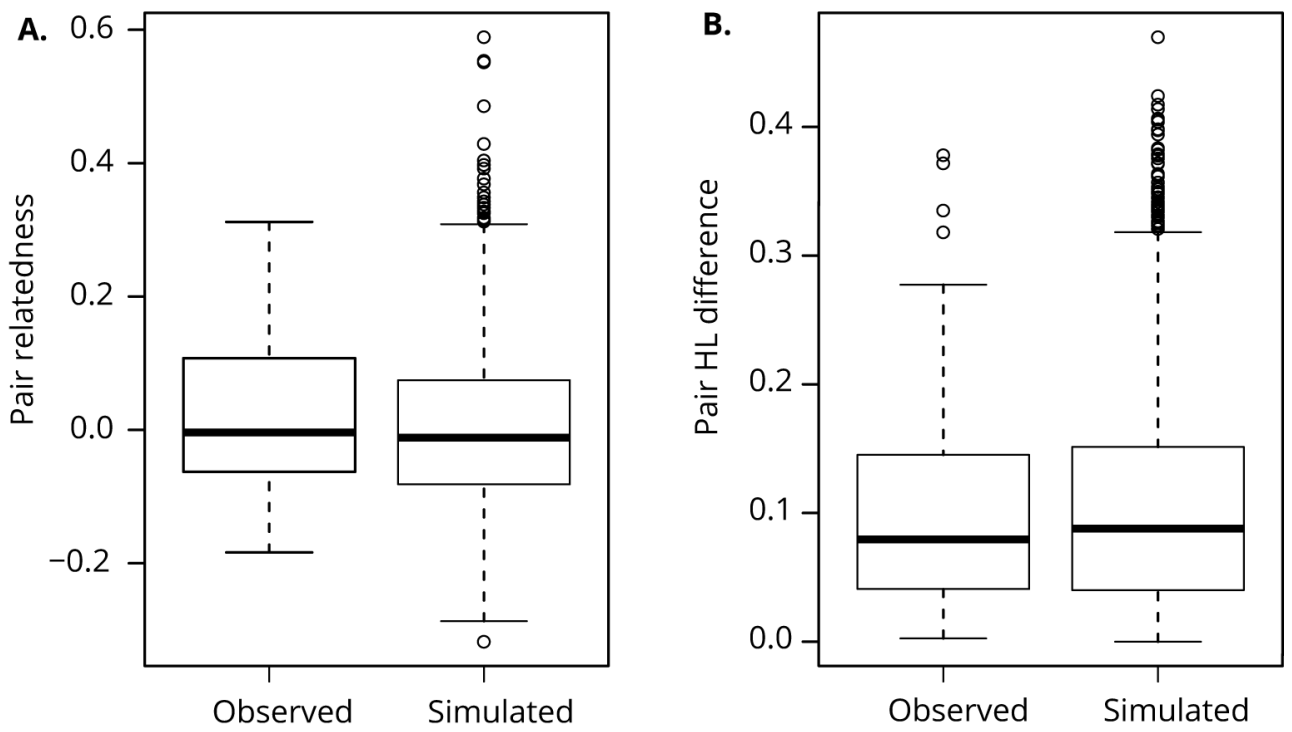

Figure S1. Distributions of the observed versus the simulated pair relatedness $(\boldsymbol{A})$ and pair difference in heterozygosity $(\boldsymbol{B})$.

\section{B. Accounting for spatial structure in mating patterns}

Although the study site was relatively small, there is the possibility that individuals were constrained in their choice for a mate by the locally available potential mates. To check this, we also compared the existing mating pattern to a different null model of random mating; one which considers the local mate availability. Using the 'nearest neighbour scenario' as used in Szulkin (2009), we paired the male and female of every pair to a known individual of the opposite sex breeding in the nest box nearest to the focal pair and compared the observed mating pattern with the simulated pattern. We tested for differences in the relatedness and heterozygosity similarity between the observed and simulated scenario of random mating using a non-parametric Wilcoxon matched-pair test. We found no differences between the observed and the simulated mating patterns, not for heterozygosity (WSR: females, $\mathrm{T}+=829, \mathrm{n} 1=\mathrm{n} 2=63, \mathrm{P}=0.53$; males, $\mathrm{T}+=969, \mathrm{n} 1=\mathrm{n} 2=63, \mathrm{P}=0.87$ ) or for relatedness (WSR: females, $\mathrm{T}+=3616, \mathrm{n} 1=\mathrm{n} 2=63, \mathrm{P}=0.43$; males, $\mathrm{T}+=900.5, \mathrm{n} 1=\mathrm{n} 2=63$, $\mathrm{P}=0.91)$.

\section{Accounting for effects of captures on mating patterns}

By capturing and bringing the focal birds into captivity we may have unintentionally affected pair bonds and in extreme cases even split up pairs. To check whether testing the birds had any effect on mate choice we also compared mating patterns between untested pairs and pairs in which one or both were brought to the lab for testing. However, the 
mating patterns in these two groups did not differ. Both tested and untested birds did not mate differently from random mating in both heterozygosity and relatedness.

Heterozygosity (Pairs of which one or both were tested: random correlation 95\% confidence interval $=[-0.27,0.28]$; correlation breeding pairs $=-0.12 ; \mathrm{N}=52$ breeding pairs; Untested pairs: random correlation $95 \%$ confidence interval $=[-0.45,0.48]$; correlation breeding pairs $=-0.36 ; \mathrm{N}=18$ breeding pairs. 10000 permutations).

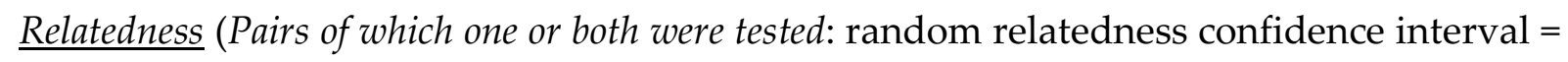
[-0.035, 0.02]; average observed relatedness breeding pairs $=0.018 ; \mathrm{N}=52$ breeding pairs; Untested pairs: random relatedness confidence interval $=[-0.020,0.067]$; average observed relatedness breeding pairs $=0.058 ; \mathrm{N}=18$ breeding pairs; 10000 iterations).

\section{Alternative parametrization of mixed models}

\section{Results mate preference based on categorized heterozygosities}

As described in Appendix S1-C we performed an extra analysis to check whether the specification of the interaction of continuous variables is not too restrictive, we also modelled the effect of these variables after categorization of each into three groups, based upon tertiles. First, the focal bird's and stimulus bird's heterozygosities were categorized into three groups based on tertiles. For the focal bird's heterozygosity we took the cut points -0.035767 and 0.064233 . For the stimulus bird's heterozygosity we took the cut points -0.028627 and 0.051373. Next, in the GLMM we replaced the continuous heterozygosities and their interaction by main effects and interaction of the grouped heterozygosities. Results after removal of non-significant terms are given in table S3.

Table S3. Full model mate preference based on categorized heterozygosities. Table consists of all factors tested in the binomial mixed model with proportion of time spent with each of the stimulus birds as the dependent variable ( $N$ focals=116, $N$ tests=359). Given is the degrees of freedom (numerator $d f$ and denominator $d f$ ), the test statistic (F value) and the significance ( $P$ value).

\begin{tabular}{lcccc}
\hline & Num df & Den df & F value & P value \\
\hline HLfocal (grouped) & 2 & 252.70 & 1.54 & 0.22 \\
HLstimulus (grouped) & 2 & 142.10 & 0.71 & 0.49 \\
HLfocal $^{*}$ HLstimulus (both grouped) & 4 & 207.00 & 3.01 & $\mathbf{0 . 0 2}$ \\
Relatedness & 1 & 65.12 & 2.15 & 0.15 \\
Relatedness2 & 1 & 287.30 & 4.23 & $\mathbf{0 . 0 4}$ \\
Relatedness ${ }^{*}$ HLstimulus & 1 & 273.80 & 5.21 & $\mathbf{0 . 0 2}$ \\
\hline
\end{tabular}


The estimated mean response (on the logit scale; mean \pm se) for the combination of focal and stimulus heterozygosities, at the average value of relatedness (i.e. value zero for centred relatedness). We have also given the back transformed mean responses, which indicate fractions of time a focal bird spent on a specific stimulus bird. Without preference the fractions would be $1 / 6=0.167$, as the focal bird can choose from six alternative stimulus birds.

Table S4. Least square means for combinations of levels of grouped focal heterozygosity and grouped stimulus heterozygosity ( \pm error) (A). Mean responses back transformed back to the probability (preference) scale for combinations of levels of grouped focal heterozygosity and grouped stimulus heterozygosity (B).

A.

\begin{tabular}{c|c|c|c}
3 & $\begin{array}{c}-1.84 \\
\text { 3 }\end{array}$ & $\begin{array}{c}-1.77 \\
\pm 0.10\end{array}$ & $\begin{array}{c}-1.65 \\
\pm 0.11\end{array}$ \\
\hline \multirow{2}{*}{2} & -1.68 & -1.56 & -1.60 \\
& \pm 0.07 & \pm 0.06 & \pm 0.07 \\
\hline \multirow{2}{*}{1} & -1.55 & -1.68 & -1.72 \\
& \pm 0.08 & \pm 0.08 & \pm 0.09 \\
\hline \multirow{2}{*}{} & 1 & 2 & 3
\end{tabular}

B.

\begin{tabular}{c|r|r|r}
3 & 0.14 & 0.15 & 0.16 \\
\hline 2 & 0.16 & 0.15 & 0.17 \\
\hline 1 & 0.18 & 0.16 & 0.15 \\
\hline & 1 & 2 & 3
\end{tabular}




\section{Results offspring fledging probability based on categorized heterozygosities}

First, the foster mother, foster father and biological mother heterozygosities and biological relatedness were categorized into three groups based on tertiles. For the foster mother heterozygosity we took the cut points -0.02536803 and 0.03791166 . For the foster father heterozygosity we took the cut points -0.03623277 and 0.06356268 . For the biological mother heterozygosity we took the cut points -0.01245976 and 0.03742855 . And for the biological relatedness we took the cut points -0.06911875 and 0.04628325 . Next, in the GLMM we replaced the continuous heterozygosities and relatedness values and their interaction by main effects and interaction of the grouped heterozygosities and relatedness. Results after removal of non-significant terms are given in table S4.

Table S5. Full model offspring fledging probability based on categorized heterozygosities. Table consists of all factors tested in the binary mixed model with the fledging probability of the offspring (0/1) as the dependent variable $(N=272)$. Given is the degrees of freedom ( $d f)$, the test statistic ( $\chi^{2}$ value) and the significance (P value).

\begin{tabular}{lccc}
\hline & df & $\chi^{\mathbf{2}}$ value & P value \\
\hline HLfoster female (grouped) & 2 & 1.96 & 0.38 \\
HLfoster male (grouped) & 2 & 2.11 & 0.35 \\
HLbiological male & 1 & 1.90 & 0.17 \\
HLbiological female (grouped) & 2 & 0.91 & 0.64 \\
Biological relatedness (grouped) & 2 & 2.08 & 0.35 \\
Offspring sex & 1 & 0.85 & 0.36 \\
Year & 1 & 0.03 & 0.87 \\
Hatch date & 1 & 3.15 & 0.08 \\
Brood size & 1 & 1.64 & 0.2 \\
HLfoster female * HLfoster male (both grouped) & 4 & 18.97 & $\mathbf{0 . 0 0 0 8}$ \\
HLbiological female * biological relatedness (both grouped) & 4 & 13.12 & $\mathbf{0 . 0 1}$ \\
\hline
\end{tabular}

The estimated mean response (on the logit scale; mean \pm se) for the combination of foster mother and foster father heterozygosities. We have also given the back transformed mean responses, which indicate the fledging probability for the combination of foster mother and foster father heterozygosity. 
Table S6. Least square means for combinations of levels of grouped foster mother and foster father heterozygosities ( \pm error) (A). Mean responses back transformed back to the probability (of fledging) scale for combinations of levels of grouped foster mother and foster father heterozygosities (B).

A.

\begin{tabular}{c|c|c|c}
3 & $\begin{array}{c}-0.73 \\
\pm 1.25\end{array}$ & $\begin{array}{c}1.60 \\
\pm 0.98\end{array}$ & $\begin{array}{c}1.64 \\
\pm 1.60\end{array}$ \\
\hline 2 & -0.58 & 0.80 & 1.99 \\
\pm 0.98 & \pm 1.13 & \pm 1.11 \\
\hline \multirow{2}{*}{1} & 1.66 & 0.91 & -3.51 \\
& \pm 1.09 & \pm 1.19 & \pm 1.09 \\
\hline & 1 & 2 & 3
\end{tabular}

B.

\begin{tabular}{c|c|c|c}
3 & 0.33 & 0.83 & 0.84 \\
\hline 2 & 0.36 & 0.69 & 0.88 \\
\hline 1 & 0.84 & 0.71 & 0.03 \\
\hline & 1 & 2 & 3
\end{tabular}

The estimated mean response (on the logit scale; mean \pm se) for the combination of biological mother heterozygosity and her relatedness with the biological father. We also give the back transformed mean responses, which indicate the fledging probability for the combination of foster mother and foster father heterozygosity.

Table S7. Least square means for combinations of levels of grouped biological mother heterozygosity and the grouped relatedness with the biological father ( \pm error) (A). Mean responses back transformed back to the probability (of fledging) scale for combinations of levels of grouped biological mother heterozygosity and the grouped relatedness with the biological father (B).

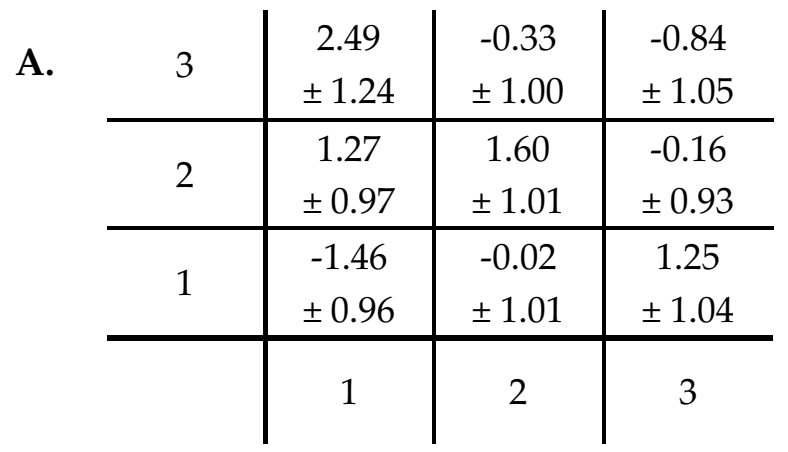

B.

\begin{tabular}{c|c|c|c}
3 & 0.92 & 0.42 & 0.30 \\
\hline 2 & 0.78 & 0.83 & 0.46 \\
\hline 1 & 0.19 & 0.49 & 0.78 \\
\hline & 1 & 2 & 3
\end{tabular}




\section{Appendix S4: Additional results with weak significance}

\section{A. Mate preferences}

Both males and females tended to spend more time with moderately related individuals than with very related or unrelated individuals (relatedness ${ }^{2}$, GLMM: $\mathrm{F}_{1,274.2}=-2.39, \mathrm{P}=0.02$ ). This effect was also influenced by the heterozygosity of the stimulus bird (relatedness*

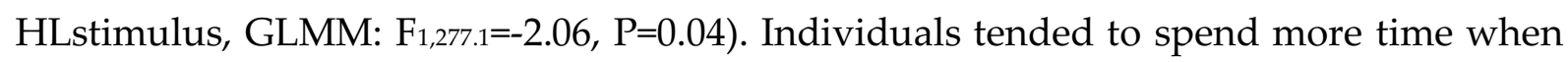
the stimulus bird was heterozygous and relatively unrelated or when the stimulus bird was homozygous and relatively related.

Individuals tended to prefer a moderately related mate rather than a genetically dissimilar mate with whom they could increase offspring heterozygosity (see also Kleven et al. 2005; Oh \& Badyaev 2006). It has been suggested that such patterns can occur when individuals balance any potential costs and benefits of in- and outbreeding by finding a mate with the 'optimal' genetic similarity, (Neff 2004; Greeff et al. 2009; Richard et al. 2009). We did not find any fitness effects of this preference. These genetic effects may be very small, or only appear later in life, and because of this we did not see them reflected in the reproductive success. Although we did find a rearing effect of parental relatedness on chick weight, this effect was the inverse of the preference tendency. Possibly this preference was a genetic preference for indirect benefits or optimal outbreeding (Bateson 1983), despite the negative direct effects of this preference on chick weight.

\section{B. Reproductive success}

Heterozygous foster fathers tended to raise heavier offspring (HLfoster male, LMM: $\mathrm{t}_{29.13}=2.24, \mathrm{P}=0.03$ ). Biological fathers (within-pair or extra-pair sire), which were more homozygous tended to produce offspring with a higher fledging probability (HLbiological male, GLMM: $\mathrm{Z}=-2.07, \mathrm{P}=0.04)$.

Heterozygous parents have previously been shown to invest more in their offspring (Foerster et al. 2003; García-navas et al. 2009). Heterozygous blue tits for instance provisioned more than homozygous individuals (García-navas et al. 2009). Here we found that the absolute heterozygosity levels of foster fathers tended to be correlated with a higher fledging weight, which indeed suggests that these males may be able to invest more resources in their offspring and give direct reproductive benefits (García-navas et al. 2009). Females on the other hand often provision more than males and are often more responsive to male provisioning levels and chick begging. Because of this we may not see the same correlation with heterozygosity as in males. Conversely, the heterozygosity levels of the biological fathers tended to have a negative effect on fledging success. Possibly homozygous birds and offspring are better locally adapted to the environment and 
heterozygous males were more likely to sire offspring that is less adapted. Apart from genetic effects the higher fledging success might also work through other pre-hatching effects that may be related to male attractiveness, such as maternal investment in egg size (Cunningham and Russell 2000; Horváthová et al. 2012), yolk carotenoids (Marri and Richner 2014) or yolk androgens (Gil et al. 1999; Kingma et al. 2009) 


\section{Appendix S5 Full statistical models}

Table S8 - Mate preferences full model. Table consists of all factors tested in the binomial mixed model with proportion of time spent with each of the stimulus birds as the dependent variable ( $N$ focals=116, $N$ tests=359). Given is the degrees of freedom (df), the test statistic (F value) and the significance (P value). A random effect for stimulus bird identity (mean $\pm S E ; 0.23 \pm 0.05$ ) and random slopes for focal bird identity with respect to the stimulus birds heterozygosity $(2.37 \pm 0.76)$, relatedness $(1.31 \pm 0.51)$, and offspring heterozygosity $(3.21 \pm 2.09)$, and a random effect for test number (to allow for negative correlations among association times within one six-choice test; $(-13.60 \pm 0.51)$ and an extra scale parameter on the original scale, were included in the model. Degrees of freedom for F-tests were calculated using the degree of freedom approximation proposed by Kenward and Roger (1997).

\begin{tabular}{|c|c|c|c|c|}
\hline & Num df & Denom df & F value & P value \\
\hline HLfocal & 1 & 191.50 & 2.76 & 0.10 \\
\hline HLstimulus & 1 & 164.40 & 0.42 & 0.52 \\
\hline HLfocal $^{*}$ HLstimulus & 1 & 62.56 & 6.47 & 0.01 \\
\hline Relatedness & 1 & 124.40 & 2.37 & 0.13 \\
\hline Relatedness ${ }^{2}$ & 1 & 293.00 & 3.29 & 0.07 \\
\hline Relatedness * HLstimulus & 1 & 278.50 & 4.19 & 0.04 \\
\hline HLoffspring & 1 & 69.07 & 0.00 & 0.99 \\
\hline Sex (female) & 1 & 75.29 & 0.00 & 0.98 \\
\hline HLstimulus * sex (female) & 1 & 164.40 & 1.22 & 0.27 \\
\hline HLfocal $^{*}$ HLstimulus ${ }^{*}$ sex (female) & 1 & 61.55 & 1.11 & 0.30 \\
\hline Relatedness * sex (female) & 1 & 108.10 & 0.93 & 0.34 \\
\hline Relatedness $^{2 *}$ sex (female) & 1 & 293.80 & 0.37 & 0.54 \\
\hline HLoffspring ${ }^{*}$ sex (female) & 1 & 45.21 & 0.79 & 0.38 \\
\hline
\end{tabular}


Table S9 - Offspring fledging probability full model. Table consists of all factors tested in the binary mixed model with the fledging probability of the offspring $(0 / 1)$ as the dependent variable $(N=272)$. Random effects for biological brood (var $\pm s d: 0.00 \pm 0.00)$ and foster brood (3.72 \pm 1.93$)$ were included in the model. Given is the test statistic ( $\mathrm{Z}$ value) and the significance ( $P$ value). Biological and foster brood identity were included as random factors.

\begin{tabular}{lcc}
\hline & Test statistic & P value \\
\hline HLoffspring & 0.30 & 0.76 \\
HLfoster female & -1.71 & 0.09 \\
HLfoster male & 1.09 & 0.28 \\
HLfoster female * HLfoster male & 3.42 & $<\mathbf{0 . 0 0 1}$ \\
Foster relatedness & 1.44 & 0.15 \\
HLfoster female * Foster relatedness & 0.80 & 0.43 \\
HLfoster male * Foster relatedness & 0.38 & 0.70 \\
HLbiological female & -1.99 & 0.047 \\
HLbiological male & -1.44 & 0.15 \\
HLbiological female * HLbiological male & -0.08 & 0.93 \\
Biological relatedness & 2.10 & 0.036 \\
HLbiological female * biological relatedness & -3.06 & $<\mathbf{0 . 0 1}$ \\
HLbiological male * biological relatedness & -0.73 & 0.47 \\
Offspring sex (male) & -0.67 & 0.51 \\
Brood size & -0.99 & 0.32 \\
Hatch date & 1.07 & 0.28 \\
Year (2015) & 0.10 & 0.92 \\
\hline
\end{tabular}


Table S10 - Offspring fledging weight full model. All factors tested in the linear mixed model with the fledging weight $(\mathrm{mg})$ of the offspring as the dependent variable ( $N=205)$. Biological (var \pm sd: $0.00 \pm 0.00$ ) and foster brood identity $(17943 \pm 134.0)$ were included as random factors (residual variation $=28767 \pm$ 169.6). Given is the estimate, the degrees of freedom (df), the test statistic ( $t$ value) and the significance ( $P$ value). Given are the $P$ values, $d f$ and test statistics. Degrees of freedom for $F$ - and $t$-tests were calculated using the degree of freedom approximation proposed by Kenward and Roger (1997).

\begin{tabular}{|c|c|c|c|}
\hline & df & Test statistic & P value \\
\hline Intercept & 30.78 & - & - \\
\hline HLoffspring & 118.02 & -0.04 & 0.97 \\
\hline HLfoster female & 21.45 & -0.64 & 0.53 \\
\hline HLfoster male & 19.52 & 1.46 & 0.16 \\
\hline HLfoster female ${ }^{*}$ HLfoster male & 20.17 & 1.15 & 0.27 \\
\hline Foster relatedness & 23.23 & -1.48 & 0.15 \\
\hline Foster relatedness ${ }^{2}$ & 22.04 & 2.81 & 0.01 \\
\hline HLfoster female ${ }^{*}$ foster relatedness & 24.84 & -0.66 & 0.51 \\
\hline HLfoster male ${ }^{*}$ foster relatedness & 22.42 & 0.86 & 0.40 \\
\hline HLbiological female & 97.22 & -0.44 & 0.66 \\
\hline HLbiological male & 109.05 & -1.30 & 0.20 \\
\hline HLbiological female ${ }^{*}$ HLbiological male & 48.27 & 0.14 & 0.89 \\
\hline Biological relatedness & 109.19 & 1.94 & 0.05 \\
\hline Biological relatedness ${ }^{2}$ & 134.51 & -1.56 & 0.12 \\
\hline HLbiological female ${ }^{*}$ biological relatedness & 124.03 & -1.99 & 0.05 \\
\hline HLbiological male ${ }^{*}$ biological relatedness & 96.70 & -1.26 & 0.21 \\
\hline Offspring sex (male) & 123.90 & -2.42 & 0.02 \\
\hline Brood size & 30.77 & 1.39 & 0.18 \\
\hline Catch date & 31.16 & 1.23 & 0.23 \\
\hline Year (2015) & 30.50 & 0.14 & 0.89 \\
\hline Year $(2015) *$ catch date & 31.58 & -1.06 & 0.30 \\
\hline
\end{tabular}



Chapter 4

Estimating individual mate attractiveness and the reproductive benefits of compatibility.

Lies Zandberg, Kees van Oers, Camilla A. Hinde 


\begin{abstract}
Compatibility between pair mates is known to increase reproductive success and may be especially important in species where individuals vary in their preferences for a mate. In the case of population-wide mate preferences, reproductive success and reproductive investment are known to change depending on the attractiveness of an individuals' mate. However, in species where individuals vary in their preferences, to our knowledge, no study so far has estimated a measure of pair compatibility. Here we attempt to estimate this via mate preference, to quantify the extent to which pair mates have the trait values that are preferred by their partner. Next, we investigated whether the attractiveness of an individual, as perceived by its mate, increased the reproductive success of the pair. Using repeated mate preference tests, we found that individual great tits differed in their preference slopes for several traits in a mate. On the basis of these tests we calculated preference functions for each individual and in the subsequent breeding season estimated a measure of how attractive each individual perceived the mate they were paired with. Here we found that while individuals differed in their preference for a mate, they were not more likely to obtain a mate that they perceived as attractive than under random mating. Additionally, we found no effects of perceived mate attractiveness on female reproductive investment, offspring weight or fledging probability. Thus, although with a limited sample size, we found no effect of our measure individual mate attractiveness on reproductive success, this method of estimating preferences and individual mate attractiveness gives the potential for further studies studying pair compatibility and reproductive success. A better understanding of preferences for compatibility rather than quality and the benefits related to this may shed light on the adaptive value of individual differences in preference for mates.
\end{abstract}




\section{Introduction}

By choosing the right partner individuals can gain reproductive benefits and can increase their reproductive success (Darwin 1871; Andersson 1994; Petrie 1994). These benefits can be direct, when the offspring's quality or quantity is increased by the behaviour or investment of the mate, or indirect, when the quality of the offspring is increased by the genetic contribution of the mate (Tregenza and Wedell 2000). While previously it was often thought that these benefits were primarily caused by the higher quality of these preferred partners, studies are beginning to suggest that it may be partner compatibility, rather than, or in addition to absolute partner quality, that increases reproductive success (Drickamer et al. 2003; Gowaty et al. 2003; Spoon et al. 2006; Ihle et al. 2015; Martin-Wintle et al. 2015; chapter 3 ).

Mate compatibility may be especially important when individuals differ in their preferences for a mate. When female preferences are uniform, directional selection can lead to the evolution of male traits signalling male quality. However, although uniform preferences have often been assumed, an increasing number of studies indicate that individuals differ in their preference for different trait values in a mate (Forstmeier and Birkhead 2004; Lehtonen and Lindström 2008; Holveck and Riebel 2010; Ihle et al. 2015; chapter 3). Moreover, these differences in preference can depend on the chooser's own trait values (Mays and Hill 2004; Holveck and Riebel 2007; chapter 3). Where some studies have found that individuals prefer a mate that is similar to themselves chapter 3 ), others suggest that a partner with traits that complement the choosers' traits would be beneficial (Tregenza and Wedell 2000; Szulkin et al. 2009). These studies show that individuals may prefer a mate that is the best fit for them, rather than the universally 'best individual'. Socially monogamous species are especially likely to have evolved individualistic rather than uniform preferences, due to the fact that individuals become unavailable after pairing. In these monogamous systems, strong preferences for attractive individuals may not be favoured by selection, since scramble competition over attractive mates leads to increased costs of choosiness which may not outweigh the benefits (DechaumeMoncharmont et al. 2016; Wang et al. 2017). Instead of uniform preferences it may therefore be more beneficial to have individual preferences for compatibility that lead to optimal direct or indirect benefits (Ihle et al. 2015; chapter 3).

Individuals paired with their preferred partner have been found to have a higher reproductive success, regardless of their partners quality or trait values (Drickamer et al. 2003; Gowaty et al. 2003; Thle et al. 2015; Martin-Wintle et al. 2015; chapter 3). This suggests that individual variation in mate preferences and mate compatibility can have consequences for the rate and direction of sexual selection and may play a bigger role in 
the evolution of sexually selected traits than was previously thought. However, the mechanisms behind these effects of partner compatibility on reproductive success are not yet clear.

There may be different mechanisms through which compatible parents may gain higher reproductive success. Firstly, preferred individuals may be genetically more compatible through which they obtain indirect benefits of a higher quality (genetically) offspring (Tregenza and Wedell 2000; Szulkin et al. 2009). Since heterozygosity is known to affect fitness (reviewed in Kempenaers 2007 and Chapman et al. 2009) it would benefit parents to find a partner with whom they would have very heterozygous offspring (Tregenza and Wedell 2000; Tomiuk et al. 2007; Szulkin et al. 2009). Thus, these indirect benefits of choice for a compatible partner can increase their reproductive success.

Secondly, a potential cause of a higher reproductive success may lie in a higher phenotypic compatibility for preferred pairs, leading to direct benefits of compatibility (Royle et al. 2010). This pair compatibility may be especially important for species with biparental case. Depending on the choosers' own traits, some traits or benefits may be more important to have in a mate than others. However, if and how choosers differ in the attention they pay to different mate qualities and how they weigh different signals of quality has received relatively little attention (Candolin 2003). Especially since some of these traits are known to be negatively correlated these decisions for multiple traits in a mate may be a trade-off. For instance, aggressive or bold males often obtain better territories (Both et al. 2005), but also provision their offspring less (Barnett et al. 2012; Mutzel et al. 2013). For a female that is very good at provisioning her offspring, an aggressive male that provisions less, but that can obtain a good territory can be an optimal choice. On the other hand, for a female that has lower provisioning abilities the provisioning abilities of her mate may be more important, and his dominance not so much. Thus choosers may differ in how they weigh courter traits, depending on their own traits and qualities, such as their ability to compensate for reductions in male care (see Burley 1986; see Sheldon 2000) or their genetic variation (Pilakouta and Smiseth 2017).

A third possibility is that individuals invest differently in this current reproductive attempt depending on the attractiveness of their partner (Sheldon 2000). Parents trade-off their current and future reproduction by allocating resources to reproduction over time depending on the expected costs and benefits of these current and future reproductive opportunities (Houston and McNamara 1999; Brommer 2000). The attractiveness of their mate and the reproductive benefits correlated with his traits affect the optimal trade-off between different reproductive opportunities, and thus the optimal investment by the partner (Ratikainen and Kokko 2009). Thus, individuals are thought to increase their 
investment with increasing mate attractiveness or quality, (reviewed in Sheldon 2000 and Horváthová et al. 2012). However, several studies have shown a decrease in reproductive investment with increasing mate quality (Bluhm and Gowaty 2004; Byers and Waits 2006; Gowaty et al. 2007; Bolund et al. 2009), initially termed reproductive compensation (Gowaty et al. 2007). Differential allocation and reproductive compensation may actually be the ends of a continuum of reproductive investment patterns depending on mate qualities (Harris and Uller 2009; Ratikainen and Kokko 2009). Through this process, individuals are thought to optimise their reproductive success depending on their partner, the environmental conditions and their future prospects.

Despite these many studies suggesting individual differences in preferences and the benefits of compatibility, few studies so far have estimated individual preference functions (but see McGuigan et al. 2008; Kilmer et al. 2017), let alone used individual preference functions to estimate how attractive that individual perceives its mate (to our knowledge no study has attempted this so far). On a population level, however, this approach is quite common. Based on preference tests population level preferences are estimated, after which males are valued as generally 'attractive or unattractive' depending on where on the preference function their trait value falls (Zuk et al. 1995; Galeotti et al. 2005; Holveck and Riebel 2010). Subsequently these traits are used as signals of quality or universal attractiveness and are correlated with reproductive success or reproductive investment (Cunningham and Russell 2000; Horváthová et al. 2012; Arnold et al. 2016). While in cases of uniform, population-wide preferences these methods are justified, in cases of individual variation in preferences this may lead to confounding results or an absence of a compatibility effect. A different experimental approach, which does take individual differences in preference into account, lets individuals choose between two or more stimulus individuals, after which they are paired up either with their preferred or unpreferred choice (Koeninger Ryan and Altmann 2001; Gowaty et al. 2003; Anderson et al. 2007; Ihle et al. 2015). Preferred, and thus likely compatible pairs subsequently had a higher reproductive success than unpreferred pairs, independent of the individual phenotypes and thus the individual quality. These experiments demonstrate that whether individuals obtained the mate they preferred - the one they found most attractive - had a large effect on their reproductive success regardless of what their preferences were. While this method works in experimental conditions in which pair formation can be controlled, in natural populations it is impossible to test each female for her preferences for each male in the population. In that case, an approach which relates a measure of an individuals' preferences for certain traits to the traits of the mate they obtained could be the solution to this, giving an indication of the extent to which individuals find their mate attractive or not. 
The aim of this study was to investigate whether pair compatibility - the combination of individuals that breeds most successfully together can be chosen via mate choice for the traits measured. Breeding success could be through indirect (pre-hatching) benefits, direct (post-hatching) benefits, or through (pre-hatching) differential allocation. Since individuals may differ in their preferences, the attractiveness of each individual differs depending on the preferences of its observer (Widemo and Sæther 1999). Therefore, rather than referring to the attractiveness of a potential mate, the term individual mate attractiveness' (IMA), as perceived by its observer, in this case the focal individual, is more appropriate. Here, we estimated how attractive individuals perceived their mate to be: their individual mate attractiveness (IMA score). We used preference tests to test whether individuals differed in their preferences. Subsequently we specified the preference slopes for each choosing individual for the traits heterozygosity, yellowness, breast stripe size and body condition. In the following breeding season we followed all birds that were tested for their preference, and were found breeding in our field site. On the basis of the individual preference slopes measured in the preference tests, we estimated for each individual their individual mate attractiveness score. By cross fostering chicks between broods, we assessed the direct and indirect benefits of individual mate attractiveness on reproductive success. We expected females to invest differently depending on their mates estimated individual attractiveness, through differential allocation or reproductive compensation. Additionally, we expected individuals with a mate with high individual mate attractiveness scores to have a higher reproductive success (due to high compatibility), both through direct and indirect effects. Furthermore, we expected that the individual mate attractiveness scores for the different traits may affect reproductive success in different ways. Depending on the trait, pair compatibility for one trait may have different consequences compared to compatibility for other traits, such as direct versus indirect benefits. 


\section{Methods}

\section{Preference tests}

For all details on the methods of the preference tests see chapter 3 . We performed preference experiments using wild great tits originating from six unique field sites, which were different from our own field site, to minimize the chance the stimulus birds (chosen birds) were familiar with the focal birds (choosers). The birds were housed at the NIOO-KNAW facilities where we also carried out the mate preference tests. At this facility, we attached a unique metal leg ring, weighed them and photographed their breast stripe, after which we housed them individually in standard cages $(0.9$ x $0.4 \times 0.5 \mathrm{~m})$, with a solid bottom, top,

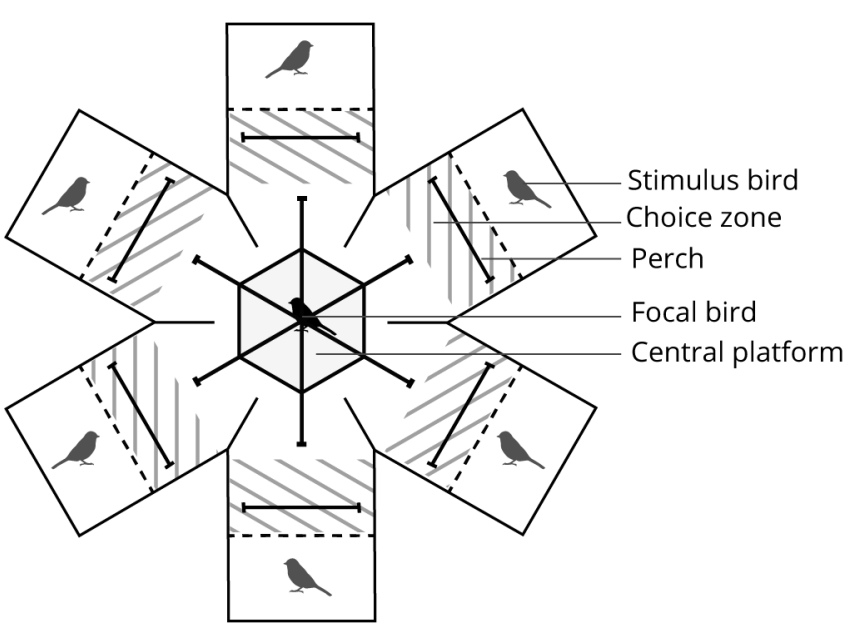

Figure 4.1 - Experimental setup mate preference test. Birds were tested for their preference in a six-choice test room. From the hexagonal central platform the focal bird could observe all stimulus birds, whereas from the perches in the choice zone only one stimulus bird was visible. Time spent in each of the choice zones was measured.

side and rear walls, a wire-mesh front and three perches. The birds had ad libitum access to water and sunflower seeds, supplemented daily with commercial egg mixture, ground peanuts, live mealworms, soldier fly larvae, dead wax moth larvae, green bottle fly larvae and crickets. Following the 6 days of preference tests, we took a blood sample (approx. 10 $\mu \mathrm{l})$ from the brachial vein, and measured weight and tarsus length.

All tests took place in a test room $(4.0 \times 2.4 \times 2.5 \mathrm{~m})$ with white walls, and high-frequency fluorescent lights to mimic natural lighting conditions (Figure 4.1). We tested individuals mate preferences using a carrousel shaped six-choice chamber (chapter 3). As great tits pair throughout the winter period (Culina 2014), we tested the birds for their mate preferences in the months of January, February and March. During the winter great tits often forage in large foraging flocks, while keeping local dominance in their breeding territories. Encountering 6 birds of the opposite sex is therefore not unrealistic for this species. Moreover with the six-choice test it is possible to measure individual directional preferences as well as quadratic (stabilising or disruptive) preferences (Edward 2015).

Each focal bird was tested with 3 different groups of 6 stimulus birds. In total we tested 69 focal females and 70 focal males for their preference with a total of 42 groups of 6 stimulus birds $(\mathrm{N}=252)$. 
The setup consisted of a central zone for the focal bird and 6 stimulus cages placed around it. The stimulus cages were separated from the central area with transparent panels, to allow visual contact between the focal bird and a stimulus bird. All birds were within auditory range of each other. In the middle of the central focal compartment we placed a hexagonal platform to prevent visual contact between the stimulus birds. On top of this we placed a perch with 6 branches of equal length in the direction of the stimulus cages, from which the focal bird could observe all stimulus birds. Two perches were present in front of every stimulus cage for the focal bird to sit on in proximity of the stimulus bird. From this position it could only observe that specific stimulus bird. The movements of the focal bird were recorded using a central camera pointing down from the ceiling (Panasonic WV-CP500). Using EthoVision XT software (Noldus Information Technology) we analysed the videos to calculate for every test the time that the focal bird spent in each choice zone.

For each test, we placed a stimulus bird in each stimulus cage and gave them 15 minutes to habituate. After introducing the focal bird into the setup we started the recording, which lasted for 45 minutes. Each focal bird was tested with 3 groups of different stimulus birds (groups A, B and C), of which one group was repeated (order of testing: ABCA). Every stimulus bird participated in 13 or 14 trials with 10 focal birds, divided over 4 mornings and afternoons over a period of six days. We randomised the positions of the stimulus birds within groups. We composed every group of stimulus birds blind with regard to their traits (natural variation). From the videos, we calculated the time spent in front of each stimulus bird, which is commonly used as a measure of preference. In numerous species association time in a choice test has been shown to predict courtship behaviours or pair formation (Clayton 1990; Hill 1990; Mays and Hill 2004; Witte 2006; Lehtonen and Lindström 2008; Dechaume-Moncharmont et al. 2011; Jeswiet and Godin 2011) and to correlate with reproductive success (Drickamer et al., 2003).

\section{Mate choice and reproductive success}

During the breeding seasons following the mate preference tests, we monitored all breeding attempts in the Boslust population, from where the focal individuals originated. Boslust is a mixed wood forest of approximately 70 ha containing 130 nest boxes. During the breeding season we checked unoccupied nest boxes every five days for nesting activity. For every nest we estimated the egg-laying date, start of incubation, hatching date and fledging date and we recorded the clutch size, mean weight of the first five eggs, brood size, and the number of fledged offspring. We weighed the chicks on the day of hatching (day 0 ) and gave them a down code by removing down feathers to individually identify each chick. These down codes were visible until at least day 6 , after which they were given an aluminium ring with a unique number. On day 1 after hatching we cross fostered the 
chicks with chicks from two other nests that had hatched on the same day. Using the cross foster design of Brinkhof et al (1999) we ranked each chick in the brood on the basis of its weight, and in the order of these ranks we sequentially re-assigned them to one of the other two nests. Using this design, all chicks were raised by foster parents. In 2014 and 2015 we cross-fostered 26 and 48 broods, with 191 and 332 chicks respectively. Chicks were weighed on day 15 after hatching (to the nearest $0.01 \mathrm{~g}$ using a digital scale) as a measure of fledging weight (Van Balen 1973), which is found to be closely correlated with first year survival (Perrins 1965). On day 3 we took a blood sample (approx. $3 \mu \mathrm{l}$ ) of all chicks from the metatarsal vein, and on day 14 the tarsus length was measured (to the nearest $0.1 \mathrm{~mm}$ using callipers). When the nestlings were approximately 10 days old, we caught the adults at the nest using spring traps. From these adults we recorded body mass, tarsus length (to the nearest $0.1 \mathrm{~mm}$ using callipers) and took a blood sample $(10 \mu \mathrm{l})$ from the brachial vein and yellow breast feathers. We fitted unringed adults with an aluminium numbered ring, and each adult with a passive integrated transponder ring (IB technology). Each nest was checked after 21-25 days for chicks that had not fledged.

\section{Traits}

\section{Yellowness}

We took a sample of 12-18 yellow breast feathers from all adult birds that were used in the preference tests $(n=391)$ or caught in the breeding season $(n=128$, of which 29 individuals were sampled in both 2014 and 2015). We obtained plumage reflectance measurements from these feather samples using the method of Quesada and Senar (Quesada and Senar 2006). Here it was shown that all colour measurements stabilise and are comparable to measurements on live birds if measures are taken from more than 9 feathers. Measurements were done using a field portable spectrometer (Jaz; Ocean Optics), a xenon light source (Jaz-PX; Ocean Optics) and a bifurcated fibre-optic probe fitted with a cylindrical probe holder at the tip to standardize measuring distance $(8.0 \mathrm{~mm})$ and to exclude ambient light (Andersson and Prager 2006). We held the probe perpendicular to the surface of the feathers during the measurements. To imitate the plumage surface of the bird with the feather sample, we stacked the 12 feathers by superimposing four layers of three feathers on a black velvet surface (Quesada and Senar 2006). We calculated reflectance measures relative to a white tile using SPECTRASUITE v. 2.0.162. In total we took nine readings from the feathers, each of which in itself was an average of 12 scans, while rearranging the stack of feathers after every third measurement. Subsequently we calculated an overall mean reflectance spectrum for the avian visible spectrum (taken to be 320-700nm; Andersson and Prager, 2006). Using the TETRACOLORSPACE (Stoddard 
and Prum 2008) we calculated the photon catch of each colour cone type; ultraviolet sensitive or violet (UVS); short-wavelength-sensitive or blue (SWS); medium-wavelength sensitive or green (MWS) and long-wavelength-sensitive or red (LWS) (Stoddard and Prum 2008). On the basis of these cone catches we calculated the SWS ratio, as a measure of intensity of the yellow plumage (Evans et al. 2010) using the following formula: 3${ }^{1}(U V S+M W S+L W S) / S W S$.

\section{Breast stripe size}

We took photos of all adult birds tested in the mate choice tests, and of those caught during the breeding season. The camera (Casio EX-H30) was mounted on a portable copy stand maintaining a standard distance to the bird. While taking the photo of the breast stripe, we held the bird outstretched by its tarsi and beak on top of graph paper as a reference (Figuerola and Senar 2000). We defined breast stripe area as the area of the black band between the point of inflexion where the ventral stripe widens to a throat patch and the posterior end of the stripe. The area was calculated in square centimetres using image analysis software ImageJ (Abràmoff et al. 2004).

\section{Heterozygosity}

We genotyped all birds that participated in the mate preference experiments (344 individuals) across 17 polymorphic microsatellite markers. Blood samples of 9 birds tested in the mate preference tests were missing and thus omitted from the genotype analysis.

We used homozygosity by locus (HL; Aparicio et al., 2006) to estimate individual genomewide genetic diversity and calculated this measure for all genotyped individuals with the R-package GENHET (Coulon 2010). Because HL represents homozygosity instead of heterozygosity we transformed the HL values into an estimate of heterozygosity by calculating the complement of HL (1-HL). Full details on marker-characteristics and the genetic analyses can be found in chapter 3 .

\section{Body condition}

We calculated body condition as the residuals from a linear regression of mass and tarsus length.

\section{Statistical analyses}

\section{Individual differences in preference slopes}

To analyse the proportion of time spent associating with each stimulus bird we used a binomial generalised linear mixed model with a logit link function. The fixed part of the model contained as explanatory variables: stimulus and focal heterozygosity, yellowness and stripe size. For the random part of the GLMM we followed the experimental design as closely as possible, specifying the next random terms (on the logit scale): 1) random 
effects for stimulus birds, since each stimulus bird was tested repeatedly; 2) random slopes for focal birds with respect to the stimulus bird's heterozygosity, yellowness and stripe size, as each focal bird was tested multiple times. Using this specification of random slopes it is possible to test for individual differences in slope of each focal individual. Unfortunately, models with a random slope for body condition did not converge and therefore measures of body condition were not included in the model. Hence, it was impossible to estimate whether individual slopes differed for this trait. Together these random effects define the G-side covariance structure. Furthermore, for the R-side covariance structure we allowed the six proportions per test to be negatively correlated (as they sum to one per six-choice test), by introducing a compound symmetric correlation structure at the proportion scale and we introduced an extra scale parameter, because we analysed a continuous proportion, for which the binomial variance-mean relationship only holds up to a scale factor. The statistical analysis was performed using procedure PROC GLIMMIX from the SAS software system (version 9.3; SAS Institute Inc., Cary, NC). On the basis of this model we were able to test whether the preference slopes differed among individuals (for the different stimulus traits). Using a likelihood ratio test we assessed the significance of each random slope specification for the stimulus bird traits (Pinheiro and Bates 2000). Trials in which the focal bird did not visit any of the stimulus birds $(\mathrm{N}=51)$ were excluded from the analysis.

\section{Calculation of individual slopes}

On the basis of the results of these preference experiments we estimated, for each focal bird, its preference functions for heterozygosity, yellowness, stripe size and body condition. Since preferences for trait values and the importance of the different traits for these preferences differed between individuals, we estimated preference functions for each focal bird separately. We ran multiple linear mixed models for each focal individual testing the correlation between the association time and different combinations of stimulus traits. Each linear mixed model included the log-transformed proportion of the time it spent associating with each stimulus bird as the dependent variable and a random intercept specification for test number. Each model contained different combinations of the four stimulus traits (heterozygosity, yellowness, stripe size and body condition) as explanatory variables. We standardised all trait values within sex to have a similar range of traits values for each trait, and to be able to compare these values with the trait values of the mates that individuals obtained in the subsequent breeding season. Using AICc model selection we determined which stimulus traits predicted the time this focal individual spent with stimulus birds, and what the estimated slope of the preference function was (linear, quadratic or logarithmic). On the basis of AICc values we determined the model with the best fit and the models with a difference in AICc value $<2$ when 
compared to this best model. Subsequently we model-averaged the estimates for these models as a general estimate for the preference slopes of each individual (Figure 4.2). For this analysis we only used birds that showed preferences (spent time with at least one stimulus individual) in at least three tests $(n=105)$. For these statistical analyses we used the packages lme4 version 1.1 (Bates et al. 2015) and MuMIn version 1.15 (Bartón 2017) in $R$ statistical software (R Development Core Team 2015).
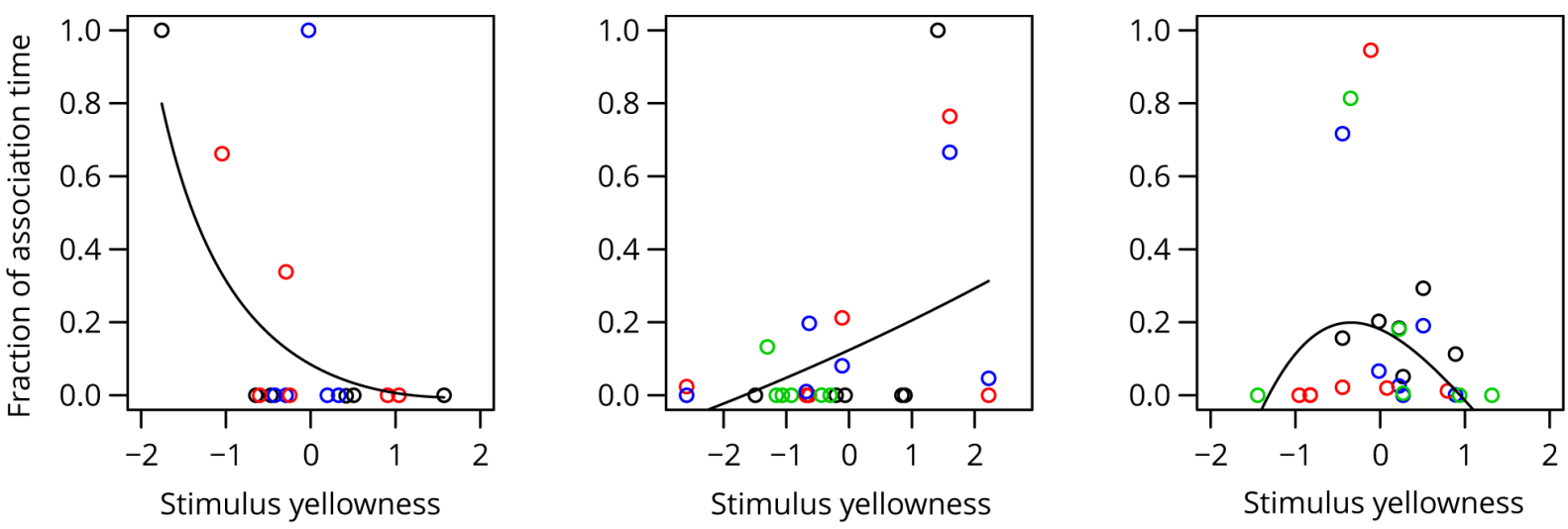

Figure 4.2 - Examples of model averaged preference slopes for stimulus yellowness on the basis of the fraction of association time of three different focal individuals. Colours indicate different tests. Stimulus yellowness values were standardised within sex.

\section{Estimating individual mate attractiveness scores}

Using these preference slopes for the standardised stimulus bird traits, we estimated for each bird that was tested for its preference and was subsequently found breeding in the field site, the perceived attractiveness of its partner, or its individual mate attractiveness (IMA) score. We obtained preference slopes for 35 breeding females and 34 breeding males. For 19 pairs we had obtained preference slopes for both parents, and for 31 pairs we had obtained the preference slope for one of the two parental birds. For each of these birds we estimated the individual mate attractiveness score on the basis of its preference slopes for each trait, and the standardised trait values of its partner. We standardised the trait values of all birds (of that sex) breeding in the field site in that year. Subsequently for every bird tested for preference function, we determined where on the estimated slope of each traits preference function the trait values of the partners lay. We could then determine the individual mate attractiveness (IMA) score by reading the proportion of time the focal individual would have spent with that bird in the preference test, on the basis of each trait. For traits that did not predict where a focal spent its time, and thus did not occur in the top models (with $\triangle \mathrm{AICc}<2$ ), we assumed a preference slope of ' 0 '. Using this approach we estimated mate attractiveness for all traits (heterozygosity, yellowness, stripe size and body condition) and we calculated the total individual mate attractiveness score as the sum of the attractiveness scores for all traits together. 


\section{Mate choice}

We tested the null hypothesis of random pairing between males and females by performing a permutation test in which we compared the mean IMA (how attractive individuals perceived their mates to be) to a sampling distribution generated by randomly permuting the mate identities within year and computing for each individual the attractiveness of these random pairings (10,000 permutations). We explored whether individuals paired differently from random with regard to total IMA and IMA for each of the four traits values.

\section{Individual mate attractiveness and reproductive success}

We then tested the correlation between our estimated values of individual mate attractiveness (IMA) and reproductive success. Using linear models we tested the effect of IMA on pre-hatching reproductive investment. We ran three linear models with lay date, clutch size and average egg weight as dependent variables. For each, we ran one model with the IMA scores for all four traits as separate explanatory variables, and we ran one model with the total IMA score as one explanatory variable. In the model on lay date we also added year as a control variable, since lay dates are known to vary between years. In the models on clutch size and egg weight we added year, lay date and the interaction between year and lay date as control variables. Additionally, in the model on egg weight we also included clutch size as a variable to control for. Non-significant terms $(\mathrm{P}>0.05)$ were deleted stepwise, starting with the highest order interactions and/or the least significant term, to obtain the minimal adequate models.

Subsequently, we tested for a correlation between the individual mate attractiveness scores and offspring fledging weight and between individual mate attractiveness score and offspring fledging probability. We tested these correlations between individual mate attractiveness and reproductive success (fledging weight and fledging probability), in separate models for biological parents and foster parents. Thus, for both biological parents and for foster parents we tested two models: One model testing a correlation between fledging weight and the total IMA and one model testing its correlation with the IMA scores for all separate traits. For this we ran two linear mixed models using the $R$ package lme4 (version 1.1-12, Bates et al. 2015), with biological brood and foster brood as random effects to account for the cross-foster design and the multiple chicks per brood. For effects of biological fathers we used the genetic sire, which in some cases was the extra-pair sire. In the first model, testing the effects of total IMA, we added total IMA, sex and the interaction between IMA and sex as explanatory variables. In the second model we added IMA for heterozygosity, for yellowness, for stripe size and for body condition as explanatory variables, as well as sex and the interactions between IMA and sex. Because 
brood size and offspring sex are known to affect chick weight we controlled for these variables in both models (sex was included as a categorical variable). We also added catch date (standardised within year), year (as a categorical variable) and the interaction between catch date and year (catch date ${ }^{*}$ year) as control variables to both models. Thus, in total, we tested four models on fledging weight: 2 models for biological parents, and two for foster parents, testing the effects of total IMA or IMA for the different traits separately). From the full model with all explanatory variables non-significant terms $(P>0.05)$ were deleted stepwise, starting with the highest order interactions and/or the least significant term, to obtain the minimal adequate models.

We tested for differences in fledging probability for each chick with a binary generalised linear mixed model using the $R$ package lme4 (Bates et al. 2015), with fledging (yes or no) as a dependent variable. Similar to the models for fledging weight we also tested two models for fledging probability for both biological parents and for foster parents: One model testing a correlation between fledging probability and the total IMA and one model testing its correlation with the IMA scores for all separate traits. In the first model, testing the effects of total IMA, we added total IMA, sex and the interaction between IMA and sex as explanatory variables. In the second model we added IMA for heterozygosity, for yellowness, for stripe size and for body condition as explanatory variables, as well as sex and the interactions between IMA and sex. We added brood size, hatch date, offspring sex and year as control variables (offspring sex and year as a categorical variables). Biological brood and foster brood were added as random effects to account for the cross-foster design. Starting with the full model with all explanatory variables, non-significant terms $(P>0.05)$ were deleted stepwise, starting with the highest order interactions and/or the least significant term, to obtain the minimal adequate models. 


\section{Results}

\section{Preference slopes}

Individuals differed in their preference slopes for heterozygosity, yellowness and stripe size. The significant random slope effects for heterozygosity, yellowness and stripe size show that individuals differ in their slopes for these different traits (table 1: likelihood ratio test; heterozygosity $\chi 2=82.55, \mathrm{P}<0.0001$; yellowness $\chi 2=22.35, \mathrm{P}<0.0001$; stripe size $\chi 2=10.84$, $\mathrm{P}=0.0005)$. Additionally the model also shows that there is no population-wide preference for these traits (Table 2: GLMM; stimulus heterozygosity: $\mathrm{F}_{1,136.4}=0.12, \mathrm{P}=0.73$; stimulus

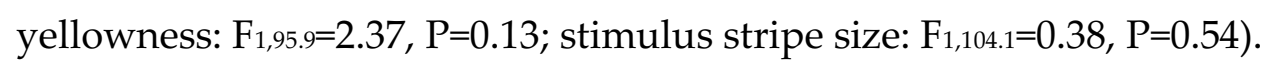

Table 1 - Likelihood ratio test random slopes for stimulus trait values: Table consists of random slopes in the binomial mixed model with proportion of time spent with each of the stimulus birds as the dependent variable tested for their significance using a likelihood-ratio test. Given are the degrees of freedom (df), the test statistic ( $\boldsymbol{X}^{2}$-value) and the significance ( $P$ value).

\begin{tabular}{lccc}
\hline Random slope & df & $\boldsymbol{\chi 2}$ & P value \\
\hline Stimulus heterozygosity I focal identity & 1 & 82.55 & $<.0001$ \\
Stimulus yellowness | focal identity & 1 & 22.35 & $<.0001$ \\
Stimulus stripe size I focal identity & 1 & 10.84 & $\mathbf{0 . 0 0 0 5}$ \\
\hline
\end{tabular}


Table 2 - Mate preferences for stimulus traits: Table consists of all factors tested in the binomial mixed model with proportion of time spent with each of the stimulus birds as the dependent variable ( $N$ focals = $116, N$ tests $=359$ ). Given are the estimate, the degrees of freedom (df), the test statistic ( $F$ value) and the significance ( $P$ value). A random intercept for stimulus bird identity (mean $\pm S E ; 0.20 \pm 0.04$ ) and random slopes for focal bird identity with respect to the stimulus bird's heterozygosity $(0.014 \pm 0.004)$, yellowness $(0.0039 \pm 0.0018)$ and stripe size $(0.004 \pm 0.002)$, and a random effect for test number (to allow for negative correlations among association times within one six-choice test; $(-13.30 \pm 0.48)$ and an extra scale parameter on the original scale, were included in the model. Degrees of freedom for $F$ - and $t$-tests were calculated using the degree of freedom approximation proposed by Kenward and Roger (1997).

\begin{tabular}{lccccc}
\hline Effect & Estimate & Num df & Denom df & F Value & P value \\
\hline Intercept & -1.67 & 1 & 47.61 & - & - \\
Stimulus heterozygosity & 0.02 & 1 & 136.4 & 0.12 & 0.73 \\
Stimulus yellowness & -0.06 & 1 & 95.86 & 2.37 & 0.13 \\
Stimulus stripe size & -0.02 & 1 & 104.1 & 0.38 & 0.54 \\
Focal heterozygosity & 0.00 & 1 & 191.8 & 0.06 & 0.81 \\
Focal yellowness & 0.00 & 1 & 162.5 & 0.42 & 0.52 \\
Focal stripe size & 0.01 & 1 & 192.2 & 0.7 & 0.40 \\
\hline
\end{tabular}

Subsequently we calculated linear preference slopes for 105 individuals for the four different traits, depending on which traits affected their association time by model averaging for the best models with $\Delta \mathrm{AICc}<2$ to obtain the predicted slope (weighted by model fit). See figure 4.3 for the preference slopes of 105 individuals for the 4 different traits. 


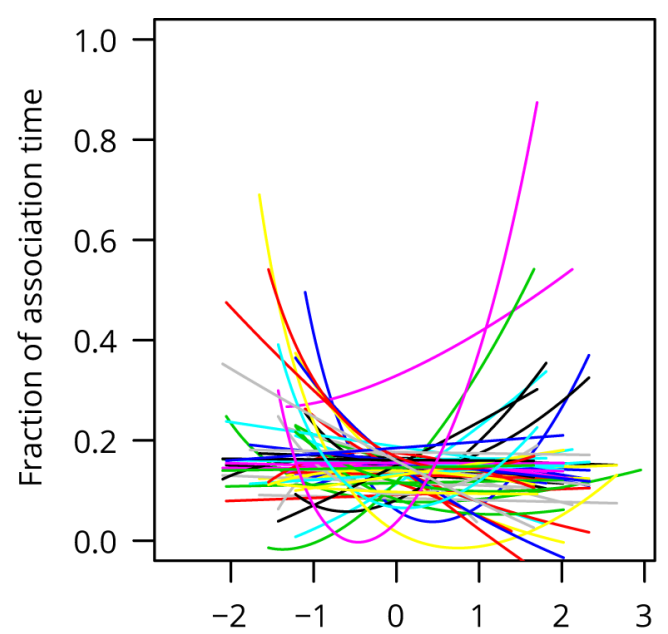

Stimulus heterozygosity (standardised)

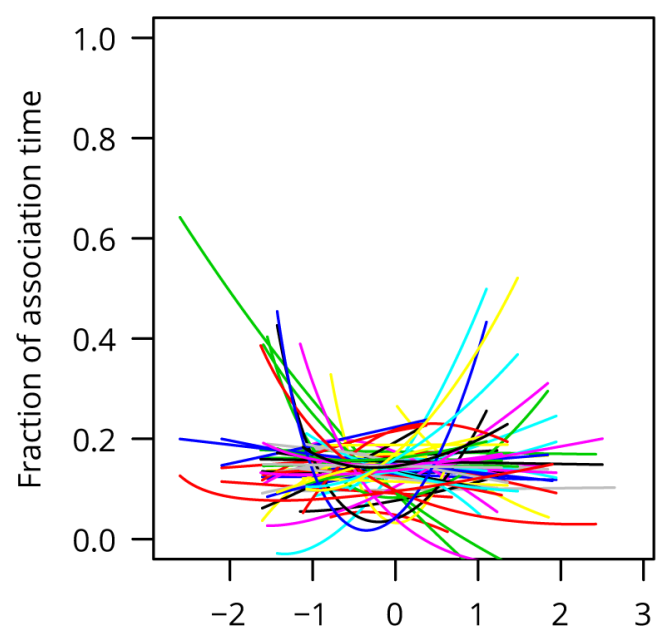

Stimulus stripe size (standardised)

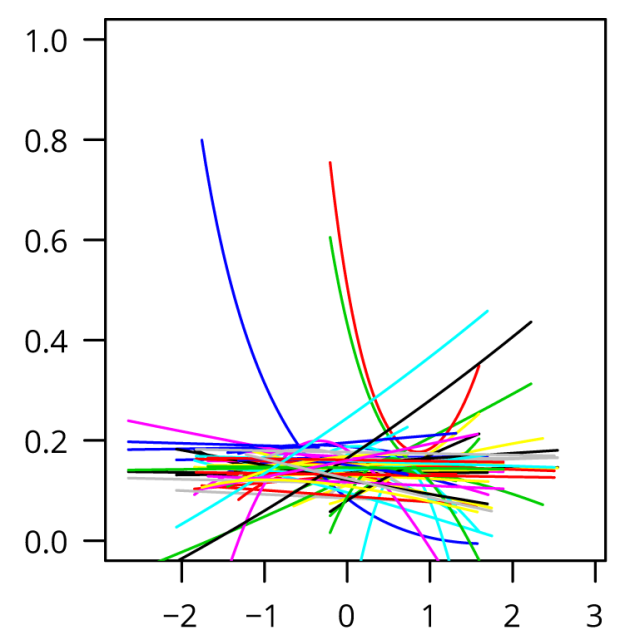

Stimulus yellowness (standardised)

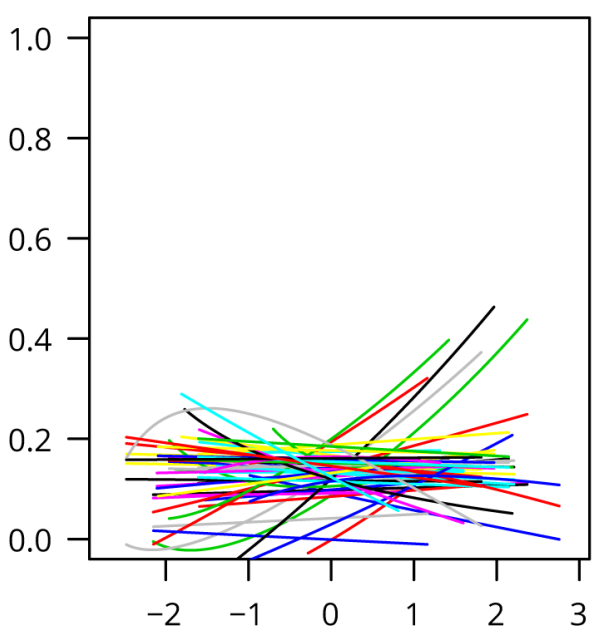

Stimulus body condition (standardised)

Figure 4.3 - Individual preference slopes for heterozygosity, yellowness, stripe size and body condition. Each line represents a different focal individual. Individual lines only cover the range of stimulus trait values they were tested with.

\section{Individual mate attractiveness}

For each individual that was tested in the mate preference tests and subsequently bred in our focal field site, we calculated a measure of how attractive they perceived their mate to be (IMA). Individuals differed in how attractive they perceived their mates to be (mean total IMA \pm SE; females: $0.42 \pm 0.03$, Figure $4.4 \mathrm{a}$; males: $0.35 \pm 0.04$, Figure $4.4 \mathrm{~b}$ ), but males and females did not differ in the average IMA score of their mate (LMM: sex: $t$ 26.11= -1.595, $\mathrm{P}=0.12$ ). Birds were not more likely to breed with individuals that had high IMA scores and they thus perceived as more attractive: there was no difference between total IMA scores within pairs and within a randomly generated distribution of pairs drawn from the population (random correlation $95 \%$ confidence interval $=[0.34,0.39]$; correlation breeding pairs $=0.38 ; \mathrm{N}=31 ; 10000$ permutations), nor did we find a difference between observed 
and random mating for either of the separate traits or for the different sexes (table S1). Individuals did not mate differently from random with regard to IMA scores. Additionally, IMA scores were not correlated with the trait values of the corresponding mates (correlation: heterozygosity: $\mathrm{t}_{64}=-0.26, \mathrm{P}=0.80$; yellowness: $\mathrm{t}_{62}=1.50, \mathrm{P}=0.14$; stripe size: $\mathrm{t}_{64}=-0.70, \mathrm{P}=0.16$, body condition: $\mathrm{t}_{63}=0.92, \mathrm{P}=0.36$ ).
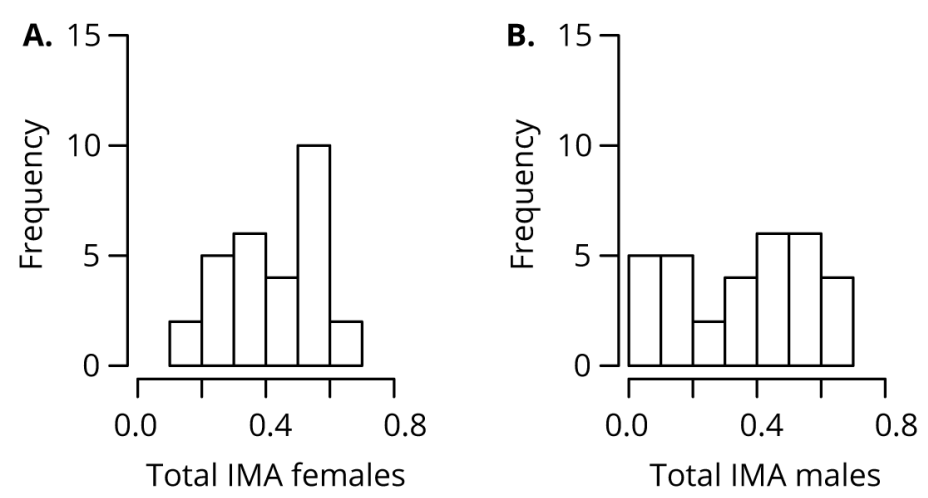

Figure 4.4-Histogram of total individual mate attractiveness scores. Graphs show individual mate attractiveness scores as perceived by females $(\boldsymbol{A})$ and males $(\boldsymbol{B})$.

\section{Biological parent attractiveness and reproductive success}

Females paired with a mate with a high IMA, did not lay earlier than females with an unattractive partner, nor did they lay larger clutches or heavier eggs (total mate attractiveness: lay date: $\mathrm{t}=0.72, \mathrm{P}=0.48$; clutch size: $\mathrm{t}=0.97, \mathrm{P}=0.34$; egg weight: $\mathrm{t}=-0.60$, $\mathrm{P}=0.56$, for full model tables see supplementary table S1-S3). Also individual mate attractiveness on the basis of the separate traits (heterozygosity, yellowness, stripe size and body condition) did not correlate with the different aspects of egg laying (lay date: IMA heterozygosity: $\mathrm{t}=0.91, \mathrm{P}=0.76$; IMA yellowness: $\mathrm{t}=0.91, \mathrm{P}=0.37$; IMA stripe size: $\mathrm{t}=-$ $0.14, \mathrm{P}=0.89$; IMA body condition: $\mathrm{t}=0.13, \mathrm{P}=0.90$; clutch size: IMA heterozygosity: $\mathrm{t}=-0.21$, $\mathrm{P}=0.83$; IMA yellowness: $\mathrm{t}=1.35, \mathrm{P}=0.19$; IMA stripe size: $\mathrm{t}=-0.40, \mathrm{P}=0.70$; IMA body condition: $\mathrm{t}=0.92, \mathrm{P}=0.37$; egg weight: IMA heterozygosity: $\mathrm{t}=0.00, \mathrm{P}=0.99$; IMA yellowness: $\mathrm{t}=0.32, \mathrm{P}=0.75$; IMA stripe size: $\mathrm{t}=1.25, \mathrm{P}=0.22$; IMA body condition: $\mathrm{t}=-1.01, \mathrm{P}=0.32$, for full model tables see supplementary information table S3-S6).

Biological parents that perceived their partners as attractive did not produce offspring with higher fledging weight or higher fledging probabilities. In biological parents IMA did not correlate with their offsprings' fledging weight (fledging weight, LMM: total IMA: $\mathrm{t}_{187.20}=-0.90, \mathrm{P}=0.37$, table S7), nor did it correlate with fledging probability (fledging probability, GLMM: total IMA: $\mathrm{Z}=0.59, \mathrm{P}=0.55$, table 3). Moreover, fledging probability and fledging weight did not differ depending on the biological parents' IMA for the different traits separately (fledging weight: see table S8; fledging probability: see table 4). 
Table 3 - Total individual mate attractiveness for biological parents and offspring fledging probability Table consists of all factors tested in the binary mixed model with the fledging probability of the biological offspring $(0 / 1)$ as the dependent variable $(N=299)$. Random effects for biological brood (var $\pm S D$ : $6.95 \pm 2.64)$ and foster brood $(2.30 \pm 1.52)$ were included in the model. Given is the estimate, the degrees of freedom (df), the test statistic ( $Z$ value) and the significance ( $P$ value). Using backwards elimination of factors, the $P$-values, $d f$ and test statistics given come from the last model in which the factor or interaction was included.

\begin{tabular}{lccc}
\hline & Estimate & Z value & P value \\
\hline Minimal adequate model & & & \\
Intercept & -0.51 & - & - \\
Brood size & -1.13 & -2.41 & $\mathbf{0 . 0 2}$ \\
\hline Dropped terms & & & \\
IMA total * parental sex (male) & 2.05 & 0.49 & 0.62 \\
Parental sex (male) & 0.01 & 0.03 & 0.97 \\
Year (2015) & 0.35 & 0.59 & 0.55 \\
IMA total & 0.08 & 0.59 & 0.55 \\
Offspring sex (male) & -0.22 & -0.95 & 0.34 \\
\hline
\end{tabular}


Table 4 - Individual mate attractiveness per trait for biological parents and offspring fledging probability. Table consists of all factors tested in the binary mixed model with the fledging probability of the biological offspring (0/1) as the dependent variable $(N=299)$. Random effects for biological brood (var \pm $S D: 5.70 \pm 2.29)$ and foster brood (2.21 \pm 1.49$)$ were included in the model. Given are the estimate, the degrees of freedom (df), the test statistic ( $Z$ value) and the significance ( $P$ value). Biological and foster brood identity were included as random factors. Using backwards elimination of factors, the P-values, $d f$ and test statistics given come from the last model in which the factor or interaction was included.

\begin{tabular}{lccc}
\hline & Estimate & Z value & P value \\
\hline Minimal adequate model & & & \\
Intercept & -0.51 & - & - \\
Brood size & -1.13 & -2.41 & $\mathbf{0 . 0 2}$ \\
\hline Dropped terms & & & \\
Parental sex (male) ${ }^{*}$ IMA yellowness & 3.78 & 0.32 & 0.75 \\
Parental sex (male) ${ }^{*}$ IMA stripe size & 7.23 & 0.78 & 0.43 \\
Parental sex (male) ${ }^{*}$ IMA body condition & -5.72 & -0.59 & 0.56 \\
Parental sex (male) ${ }^{*}$ IMA heterozygosity & 7.67 & 1.01 & 0.31 \\
Parental sex (male) & 0.13 & 0.26 & 0.79 \\
IMA stripe size & 0.63 & 0.18 & 0.85 \\
Hatch date & 0.23 & 0.38 & 0.71 \\
IMA heterozygosity & -1.15 & -0.41 & 0.68 \\
Year (2015) & 0.27 & 0.49 & 0.63 \\
IMA body condition & 2.25 & 0.62 & 0.54 \\
IMA yellowness & 2.22 & 0.80 & 0.42 \\
Offspring sex (male) & -0.22 & -0.95 & 0.34 \\
\hline
\end{tabular}




\section{Foster parent attractiveness and reproductive success}

Foster pairs in which parents perceived their partners as attractive, with a high IMA, were not more successful in rearing their offspring. IMA did not correlate with a higher fledging weight (fledging weight, LMM: total attractiveness: $\mathrm{t}_{135.49}=-0.33, \mathrm{P}=0.75$, table S9), or a higher fledging probability (fledging probability, LMM: total attractiveness: $\mathrm{Z}=0.11$, $\mathrm{P}=0.92$, table S10) for their offspring. Fledging probability and weight also did not differ depending on IMA per trait in foster parents (fledging weight: see table S12; fledging probability: see table 4)

Table 5 - Total attractiveness for foster parents and offspring fledging probability Table consists of all factors tested in the binary mixed model with the fledging probability of the foster offspring (0/1) as the dependent variable $(N=299)$. Random effects for biological brood (var $\pm S D: 0.85 \pm 0.92)$ and foster brood $(3.57 \pm 1.89)$ were included in the model. Given is the estimate, the degrees of freedom (df), the test statistic ( $Z$ value) and the significance ( $P$ value). Using backwards elimination of factors, the $P$-values, $d f$ and test statistics given come from the last model in which the factor or interaction was included.

\begin{tabular}{lccc}
\hline & Estimate & Z value & P value \\
\hline Minimal adequate model & & & \\
Intercept & -0.51 & - & - \\
Brood size & -1.13 & -2.41 & $\mathbf{0 . 0 2}$ \\
\hline Dropped terms & & & \\
IMA total * parental sex (male) & -2.13 & -0.63 & 0.53 \\
Parental sex (male) & 0.14 & 0.34 & 0.73 \\
IMA total & 0.17 & 0.11 & 0.92 \\
Year & -0.07 & -0.28 & 0.78 \\
Hatch date & 0.37 & 0.74 & 0.46 \\
Offspring sex (male) & -0.22 & -0.95 & 0.34 \\
\hline
\end{tabular}


Table 6 - Individual mate attractiveness per trait for foster parents and offspring fledging probability. Table consists of all factors tested in the binary mixed model with the fledging probability of the offspring (0/1) as the dependent variable $(N=295)$. Random effects for biological brood (var $\pm S D: 0.95 \pm 0.97$ ) and foster brood $(3.20 \pm 1.79)$ were included in the model. Given is the estimate, the degrees of freedom (df), the test statistic ( $Z$ value) and the significance ( $P$ value). Biological and foster brood identity were included as random factors. Using backwards elimination of factors, the P-values, $d f$ and test statistics given come from the last model in which the factor or interaction was included.

\begin{tabular}{lccc}
\hline & Estimate & Z value & P value \\
\hline Minimal adequate model & & & \\
Intercept & -1.44 & - & - \\
Brood size & -1.13 & -2.41 & $\mathbf{0 . 0 2}$ \\
\hline Dropped terms & & & \\
IMA body condition * parent sex (male) & -4.54 & -0.55 & 0.59 \\
IMA heterozygosity * parent sex (male) & -5.06 & -0.93 & 0.35 \\
IMA yellowness * parent sex (male) & -1.28 & -0.14 & 0.89 \\
IMA stripe size * parent sex (male) & 2.53 & 0.34 & 0.74 \\
Parent sex (male) & 0.18 & 0.46 & 0.65 \\
IMA stripe size & 2.45 & 0.80 & 0.42 \\
Offspring sex (male) & 0.24 & 0.70 & 0.48 \\
IMA yellowness & -0.04 & -0.01 & 0.99 \\
IMA heterozygosity & 0.64 & 0.23 & 0.82 \\
IMA body condition & -5.25 & -1.46 & 0.15 \\
Hatch date & 0.30 & 0.65 & 0.52 \\
Year (2015) & 1.44 & 1.34 & 0.18 \\
\hline
\end{tabular}




\section{Discussion}

Here we studied whether individuals can choose compatible mates, by estimating from mate preference tests in captivity how attractive individuals perceived their mate to be in the wild, and subsequently testing for a correlation between this individual mate attractiveness (IMA) with reproductive success. To our knowledge, this is the first study that estimates individual preference functions using choice experiments and subsequently calculates individual mate attractiveness after pairing in the wild. Preference slopes for heterozygosity, yellowness and stripe size were found to differ significantly between individuals. Moreover, by estimating individual specific preference function slopes, we were able to characterize for each focal bird which traits and which trait values determined its preferences. Here we found that while individuals differed in their preference for a mate, they were not more likely to obtain a mate that they perceived as attractive than under random mating. Additionally we found no correlation between our estimations of IMA (on the basis of the estimates of preference functions) and measures of differential pre-hatching investment and direct or indirect benefits of compatibility.

We estimated individual preference functions using a model selection approach. Since we found that great tits differed in their preference slopes for heterozygosity, yellowness and stripe size (see also chapter 3 ), we estimated preference slopes for each individual without making assumptions about the shape of the preference function or about which of the four stimulus traits predominantly determined its preferences (Edward 2015; chapter 3; Kilmer et al. 2017). Using this approach, we were able to test for preferences while allowing for directional, unimodal and bimodal preference functions in our models. Additionally we allowed for differences in the relative importance of each stimulus trait for their preference, since, depending on the choosers own traits, some traits may be more or less important to have in a mate (Pilakouta and Smiseth 2017; chapter 3). We found that the majority of our estimated preference functions were directional with an optimum at either end of the trait distribution and occasionally unimodal with an optimum preference at a certain point along the trait distribution. Surprisingly, a few preference functions were bimodal, suggesting a preference for both very large and very small trait values. Such patterns may occur when two traits are genetically negatively correlated and choosers have multivariate preferences for both traits (Candolin 2003; Burke and Murphy 2007; Prokop and Drobniak 2016; Rosenthal 2017). In this case there may be a trade-off between having very large trait values for one of the traits and with it, small trait values for the other trait. Additionally, if there are genetic covariances between different traits, the whole attractiveness may not be the same as the sum of the attractive values for the separate parts (Prokop and Drobniak 2016), which may bias our results. However, in our case we did not 
find any correlations between trait values. Another option for this pattern may lie in a measurement error due to a limited distribution of stimulus trait they were presented with. If data points are lacking along the ends of the distribution of stimulus traits quadratic or logistic model fitting may erroneously produce such bimodal preference functions. Additionally, it should be noted that, although we measured traits that we hypothesised to be of importance for great tit mate preferences, it is possible that other, unmeasured, traits also affected the preferences and with it the association times, thereby increasing the noise in our preference curves

The observed mating patterns for individual mate attractiveness did not differ from random mating scenarios. This suggests that great tits were not more likely to mate with an individual with trait values similar to those they found attractive in the preference tests. However, it is possible that individuals still choose according to their preferences from the options that they sampled, which is dependent on the availability and distribution of potential mates. Unfortunately, from our data, we cannot say anything about the availability, distribution or the number of sampled potential mates. Additionally it is possible that under social monogamy selection may not favour high levels of choosiness because the benefits of choosiness may not exceed the costs related to scramble competition (Dechaume-Moncharmont et al. 2016; Wang et al. 2017). Under such circumstances individuals are expected to have very low acceptance thresholds or to sample very few potential mates. These low levels of choosiness and sampling effort may lead to very subtle mating patterns and may explain the absence of mating patterns in our observations.

We expected to find an influence of individual mate attractiveness on the different aspects of reproductive success or reproductive investment. However, unexpectedly, we found no effects of individual mate attractiveness on reproduction at all. In a number of species with bi-parental care it has been shown that females often increase clutch size or egg weight in response to male quality, especially if male sexual traits honestly signal direct benefits (Burley 1986; Burley 1988; Sheldon 2000; reviewed in Horváthová et al. 2012). Even though these studies all assume uniform preferences and effects of general mate quality on reproductive investment, if, in the case of different preferences, females still benefit from a compatible partner, differential allocation may be expected. Therefore, it was expected that in our study females would also invest differentially in response to individual mate attractiveness, be it with a higher or a lower investment. Their response to IMA could have caused differential allocation due to a higher total IMA, but could also have depended on the IMA for the different traits, since females may respond differently to the different traits. For some male traits females may increase their reproductive investment with increasing IMA, while for other traits the IMA might not have made a difference. 
However, also for the trait specific IMA scores we found no correlation with differential investment. Possibly females still invested differentially, but only in response to the actual male trait values and the expected benefits related to these traits, rather than to how attractive they perceived their mates to be. However, if this were the case, females would still be expected to have uniform preferences only for those male traits signalling uniform benefits, which is not the case here.

Additionally, we expected in the case of preferences for compatibility, to see direct or indirect benefits of compatibility on reproductive success. Mate attractiveness, however, was not correlated with fledging weight or fledging probability. Again, this may suggest that individuals did not show preferences for mates with whom they would optimise genetic compatibility or parental care. However, while in a previous study on the same birds we found that preferences for heterozygosity were reflected in direct benefits of mating according to these preferences (chapter 3), here we do not find this correlation between mate attractiveness for heterozygosity and reproductive success. It should be noted however that the sample size is significantly smaller for these analyses, since only a limited number of the preference tested birds went on to breed in our study area. And on top of that the sample size of pairs for which we tested both pair mates for their preferences was even smaller. This limited sample size may be the reason why we did not see any effect of mate attractiveness on reproductive success, not even for heterozygosity. To test whether this limited sample size may indeed have played a role we tested the effect of heterozygosity compatibility on fledging probability, which was significant in our larger dataset, on this limited dataset as well (results not reported here). In the smaller dataset the combination of male and female heterozygosity was not significant anymore. Sample size may thus indeed play a role in the absence of a significant effect of pair compatibility. Another factor that may have played a role in the absence of an effect of compatibility on reproductive success may lie in the reliability of preference function measurements. It has previously been shown to be difficult to find repeatability for preference functions (Bell et al. 2009; Rosenthal 2017) and many external or internal factors can affect their measurement. However, while preferences may vary over environments and internal states, with increasing numbers of preference tests the chance of finding the 'real' preference increases. Possibly our estimation of preference functions, which were based on three or four tests, may still have contained too much noise to be able to characterise the real preference functions of individuals. Repeating this approach on a larger sample size, in a wild population or in a controlled captive population, may give a clearer indication whether there is scope for the use of individual preference function to estimate perceived mate attractiveness. 
Nevertheless, in light of recent findings indicating individual differences in preferences for a mate and the increase of reproductive success through compatibility, a more suitable measure of mate attractiveness and compatibility is needed. Despite the fact that in this study we did not find effects of perceived mate attractiveness, this method of estimating preferences and perceived mate attractiveness gives the potential for further studies studying pair compatibility and reproductive success. However, with this increased specificity of individual preference functions, rather than population-wide preferences, the effects of noise may be larger and because of which sample sizes may be more crucial to obtain reliable results. A better understanding of preferences for compatibility rather than quality and the benefits related to this may shed light on the adaptive value of individual differences in preference for mates and how under sexual selection variation in traits is still maintained. 
Supplement to chapter 4 


\section{Mate choice}

Table S1: Mean IMA and confidence intervals for IMA under random mating. 95\% Confidence Intervals were obtained by generating a sampling distribution by randomly permuting the mate identities within year and computing for each individual the attractiveness of these random pairings $(10,000$ permutations). This was done for both sexes together and for males and females separately.

\begin{tabular}{lcc} 
& mean IMA & $\mathbf{9 5 \%}$ CI random mating \\
\hline Total IMA & 0.384 & {$[0.34,0.39]$} \\
Female & 0.424 & {$[0.38,0.45]$} \\
Male & 0.347 & {$[0.29,0.36]$} \\
\hline IMA heterozygosity & 0.086 & {$[0.08,0.10]$} \\
Female & 0.115 & {$[0.10,0.13]$} \\
Male & 0.080 & {$[0.06,0.09]$} \\
\hline IMA yellowness & 0.086 & {$[0.08,0.10]$} \\
Female & 0.100 & {$[0.09,0.12]$} \\
Male & 0.074 & {$[0.06,0.09]$} \\
\hline IMA stripe size & 0.112 & {$[0.10,0.12]$} \\
Female & 0.113 & {$[0.10,0.13]$} \\
Male & 0.111 & {$[0.08,0.12]$} \\
\hline IMA body condition & 0.081 & {$[0.07,0.09]$} \\
Female & 0.083 & {$[0.08,0.10]$} \\
Male & 0.080 & {$[0.06,0.09]$} \\
\hline
\end{tabular}




\section{Biological parent effects}

\section{Prehatching effects}

Table S2: Total individual mate attractiveness for biological female and lay date: All factors tested in the linear model with the lay date of the first egg (counted as number of days after first of April) the dependent variable $(N=24)$. Given is the estimate, the degrees of freedom ( $d f)$, the test statistic ( t value) and the significance ( $P$ value). Using backwards elimination of factors, the P values, df and test statistics given come from the last model in which the factor or interaction was included.

\begin{tabular}{lccc}
\hline & Estimate & t value & P value \\
\hline Minimal adequate model & & & \\
Intercept & 0.31 & - & - \\
Year (2015) & 1.18 & 3.11 & $<\mathbf{0 . 0 0 1}$ \\
\hline Dropped terms & & & \\
IMA total & 0.92 & 0.72 & 0.48 \\
\hline
\end{tabular}

Table S3: Total individual mate attractiveness for biological female and clutch size: All factors tested in the linear model with the clutch size as the dependent variable $(N=24)$. Given is the estimate, the degrees of freedom (df), the test statistic ( $t$ value) and the significance ( $P$ value). Using backwards elimination of factors, the P values, df and test statistics given come from the last model in which the factor or interaction was included.

\begin{tabular}{lccc}
\hline & Estimate & t value & P value \\
\hline Minimal adequate model & & & \\
Intercept & 7.67 & - & - \\
Year (2015) & -0.06 & -0.12 & 0.91 \\
Lay date & 0.04 & 0.14 & 0.89 \\
Year (2015) ${ }^{*}$ lay date & -1.05 & -2.07 & 0.05 \\
\hline Dropped terms & & & \\
IMA total & 1.36 & 0.97 & 0.34 \\
\hline
\end{tabular}


Table S4: Total individual mate attractiveness for biological female and egg weight: All factors tested in the linear model with average egg weight of the first five eggs $(g)$ as the dependent variable $(N=24)$. Given is the estimate, the degrees of freedom (df), the test statistic ( $t$ value) and the significance (P value). Using backwards elimination of factors, the P values, $d$ f and test statistics given come from the last model in which the factor or interaction was included.

\begin{tabular}{lccc}
\hline & Estimate & t value & P value \\
\hline Minimal adequate model & & & \\
Intercept & 1.12 & - & - \\
Clutch size & 0.05 & 2.56 & $\mathbf{0 . 0 2}$ \\
\hline Dropped terms & & & \\
Year (2015) ${ }^{*}$ lay date & -0.04 & -0.60 & 0.56 \\
Lay date & 0.01 & 0.21 & 0.84 \\
IMA total & 0.13 & 0.68 & 0.51 \\
\hline
\end{tabular}

Table S5: Individual mate attractiveness per trait for biological female and lay date: All factors tested in the linear model with the lay date of the first egg (counted as number of days after first of April) the dependent variable $(N=24)$. Given is the estimate, the degrees of freedom (df), the test statistic (t value) and the significance (P value). Using backwards elimination of factors, the P values, df and test statistics given come from the last model in which the factor or interaction was included.

\begin{tabular}{lccc}
\hline & Estimate & t value & P value \\
\hline Minimal adequate model & & & \\
Intercept & -0.62 & - & - \\
Year (2015) & 1.12 & 3.61 & $<\mathbf{0 . 0 0 1}$ \\
\hline Dropped terms & & & \\
IMA stripe size & -0.51 & -0.14 & 0.89 \\
IMA body condition & 0.43 & 0.13 & 0.90 \\
IMA heterozygosity & 0.75 & 0.30 & 0.76 \\
IMA yellowness & 2.39 & 0.91 & 0.37 \\
\hline
\end{tabular}


Table S6: Individual mate attractiveness per trait for biological female and clutch size: All factors tested in the linear model with the clutch size as the dependent variable $(N=24)$. Given is the estimate, the degrees of freedom (df), the test statistic (t value) and the significance ( $P$ value). Using backwards elimination of factors, the $P$ values, $d f$ and test statistics given come from the last model in which the factor or interaction was included.

\begin{tabular}{lccc}
\hline & Estimate & t value & P value \\
\hline Minimal adequate model & & & \\
Intercept & 8.54 & - & - \\
Year (2015) & -0.19 & -0.43 & 0.67 \\
Lay date & 0.02 & 0.09 & 0.93 \\
Year (2015) * lay date & -0.95 & -2.07 & 0.05 \\
\hline Dropped terms & & & \\
IMA heterozygosity & -0.64 & -0.21 & 0.83 \\
IMA stripe size & -1.46 & -0.40 & 0.70 \\
IMA body condition & 3.07 & 0.92 & 0.37 \\
IMA yellowness & 3.90 & 1.35 & 0.19 \\
\hline
\end{tabular}

Table S7: Individual mate attractiveness per trait for biological female and egg weight: All factors tested in the linear model with average egg weight of the first five eggs $(g)$ as the dependent variable $(N=24)$. Given is the estimate, the degrees of freedom (df), the test statistic ( $t$ value) and the significance ( $P$ value). Using backwards elimination of factors, the P values, df and test statistics given come from the last model in which the factor or interaction was included.

\begin{tabular}{lccc}
\hline & Estimate & t value & P value \\
\hline Minimal adequate model & & & \\
Intercept & 1.26 & - & - \\
Clutch size & 0.05 & 2.11 & $\mathbf{0 . 0 4}$ \\
\hline Dropped terms & & & \\
Lay date * year (2015) & 0.00 & -0.01 & 0.99 \\
IMA heterozygosity & -0.01 & 0.00 & 1.00 \\
Lay date & 0.66 & 0.19 & 0.85 \\
IMA yellowness & 0.06 & 0.32 & 0.75 \\
IMA stripe size & 3.64 & 1.25 & 0.22 \\
IMA body condition & -2.73 & -1.01 & 0.32 \\
\hline
\end{tabular}




\section{Fledging weight}

Table S8: Total attractiveness for biological parents and offspring fledging weight: All factors tested in the linear mixed model with the fledging weight $(\mathrm{mg})$ of the biological offspring as the dependent variable (N=190). Biological (var $\pm s d: 399.70 \pm 19.99)$ and foster brood identity (28204.80 \pm 167.94$)$ were included as random factors (residual variation $=23962.70 \pm 154.80$ ). Given is the estimate, the degrees of freedom (df), the test statistic (t value) and the significance (P value). Using backwards elimination offactors, the P values, $d f$ and test statistics given come from the last model in which the factor or interaction was included.

\begin{tabular}{lcccc}
\hline & Estimate & $\mathbf{d f}$ & $\mathbf{t}$ value & P value \\
\hline Minimal adequate model & & & & \\
Intercept & 1487.66 & 63.30 & - & - \\
Offspring sex (male) & -63.85 & 498.00 & -4.44 & $<\mathbf{0 . 0 0 1}$ \\
\hline Dropped terms & & & & \\
Hatch date * year (2015) & 37.68 & 29.04 & 0.47 & 0.65 \\
IMA total * parental sex (male) & -84.56 & 9.63 & -0.44 & 0.67 \\
Parental sex (male) & 1.50 & 167.16 & 0.06 & 0.96 \\
Brood size & 4.68 & 33.14 & 0.15 & 0.88 \\
Catch time & 8.82 & 35.95 & 0.28 & 0.78 \\
IMA total & -74.26 & 187.20 & -0.90 & 0.37 \\
\hline
\end{tabular}


Table S9: Individual mate attractiveness per trait of biological parents and offspring fledging weight: All factors tested in the linear mixed model with the fledging weight ( $m g)$ of the biological offspring as the dependent variable $(N=190)$. Biological (var $\pm s d: 4304 \pm 65.61)$ and foster brood identity $(24134 \pm$ 155.35 ) were included as random factors (residual variation $=247000 \pm 157.16$ ). Given is the estimate, the degrees of freedom (df), the test statistic (t value) and the significance ( $P$ value). Using backwards elimination of factors, the P values, df and test statistics given come from the last model in which the factor or interaction was included.

\begin{tabular}{lcccc}
\hline & Estimate & df & t value & P value \\
\hline Minimal adequate model & & & & \\
Intercept & 1487.66 & 63.30 & - & - \\
Offspring sex (male) & -63.85 & 498.00 & -4.44 & $<\mathbf{0 . 0 0 1}$ \\
\hline Dropped terms & & & & \\
Parental sex (male) * IMA stripe size & -270.69 & 10.34 & -0.59 & 0.57 \\
Parental sex (male) ${ }^{*}$ IMA yellowness & 130.17 & 37.34 & 0.29 & 0.78 \\
Parental sex (male) * IMA heterozygosity & -20.18 & 96.47 & -0.05 & 0.96 \\
Parental sex (male) ${ }^{*}$ IMA body condition & 12.86 & 30.32 & 0.02 & 0.98 \\
Hatch date *year & 41.55 & 29.35 & 0.50 & 0.62 \\
Parental sex (male) & 1.99 & 173.89 & 0.07 & 0.95 \\
IMA stripe size & -30.99 & 185.94 & -0.17 & 0.86 \\
Brood size & 4.62 & 33.47 & 0.15 & 0.88 \\
Catch time & 8.99 & 36.70 & 0.28 & 0.79 \\
IMA yellowness & -36.30 & 85.06 & -0.20 & 0.85 \\
IMA heterozygosity & -75.33 & 177.37 & -0.43 & 0.67 \\
IMA body condition & -103.72 & 187.81 & -0.55 & 0.58 \\
\hline
\end{tabular}




\section{Foster parent effects}

\section{Fledging weight}

Table S10: Total attractiveness for foster parents and offspring fledging weight: All factors tested in the linear mixed model with the fledging weight $(\mathrm{mg})$ of the biological offspring as the dependent variable $(N=173)$. Biological (var \pm sd: $10377 \pm 101.9)$ and foster brood identity (22007 \pm 148.3$)$ were included as random factors (residual variation $=22373 \pm 149.6)$. Given is the estimate, the degrees of freedom (df), the test statistic (t value) and the significance ( $P$ value). Using backwards elimination of factors, the P values, $d f$ and test statistics given come from the last model in which the factor or interaction was included.

\begin{tabular}{lcccc}
\hline & Estimate & $\mathbf{d f}$ & $\mathbf{t}$ value & P value \\
\hline Minimal adequate model & & & & \\
Intercept & 1487.66 & 63.30 & - & - \\
Offspring sex (male) & -63.85 & 498.00 & -4.44 & $<\mathbf{0 . 0 0 1}$ \\
\hline Dropped terms & & & & \\
Hatch date * year (2015) & 3.33 & 18.19 & 0.03 & 0.97 \\
Total IMA * parental sex (male) & 0.46 & 144.26 & 0.00 & 1.00 \\
Parental sex (male) & -4.33 & 144.05 & -0.15 & 0.88 \\
Brood size & 9.84 & 51.20 & 0.39 & 0.70 \\
Catch time & -1.20 & 51.50 & -0.04 & 0.97 \\
Total IMA & -35.35 & 135.49 & -0.33 & 0.75 \\
\hline
\end{tabular}


Table S11: Individual mate attractiveness per trait of foster parents and offspring fledging weight: All factors tested in the linear mixed model with the fledging weight $(\mathrm{mg})$ of the biological offspring as the dependent variable $(N=173)$. Biological (var $\pm s d$ : $10478 \pm 102.4)$ and foster brood identity $(23167 \pm 152.2)$ were included as random factors (residual variation $=23095 \pm 152.0$ ). Given is the estimate, the degrees of freedom (df), the test statistic ( $t$-value) and the significance (P-value). Using backwards elimination of factors, the P-values, $d$ f and test statistics given come from the last model in which the factor or interaction was included.

\begin{tabular}{lcccc}
\hline & Estimate & $\mathbf{d f}$ & $\mathbf{t}$ value & P value \\
\hline Minimal adequate model & & & & \\
Intercept & 1487.66 & 63.30 & - & - \\
Offspring sex (male) & -63.85 & 498.00 & -4.44 & $<\mathbf{0 . 0 0 1}$ \\
\hline Dropped terms & & & & \\
Parental sex (male) * IMA heterozygosity & -325.38 & 114.71 & -0.73 & 0.47 \\
Parental sex (male) * IMA yellowness & -244.27 & 25.92 & -0.31 & 0.76 \\
Parental sex (male) * IMA stripe size & -106.20 & 38.55 & -0.17 & 0.86 \\
Parental sex (male) ${ }^{*}$ IMA body condition & 197.37 & 123.01 & 0.32 & 0.75 \\
Catch date *year (2015) & 46.18 & 22.48 & 0.43 & 0.67 \\
Parental sex (male) & -5.46 & 142.17 & -0.19 & 0.85 \\
IMA stripe size & 68.73 & 155.55 & 0.36 & 0.72 \\
Brood size & 9.84 & 51.20 & 0.39 & 0.70 \\
Catch time & 5.56 & 23.41 & 0.18 & 0.86 \\
IMA yellowness & -36.57 & 144.46 & -0.16 & 0.87 \\
IMA heterozygosity & 69.08 & 132.99 & 0.34 & 0.73 \\
IMA body condition & -193.16 & 161.31 & -0.83 & 0.41 \\
\hline
\end{tabular}





\section{Chapter 5}

Provisioning is affected by personality, but not the compatibility of personalities in great tit parents

Lies Zandberg, Peter Clark, Kees van Oers, Camilla A. Hinde 


\section{Abstract}

1. Under sexual selection, individuals can gain reproductive benefits by preferring certain traits in a mate. In particular, behavioural traits, such as personality traits and behavioural compatibility may be important for reproductive success, especially for species with biparental care. Indeed, behaviourally similar pairs; for instance, those with a similar personality type, have been found to have greater reproductive success than dissimilar pairs, but what causes this effect is not yet clear.

2. A possible explanation may lie in variation in provisioning behaviour, depending not only on the individuals' own personality, but also on the compatibility of personality types within the pair. Here we investigate the role of personality and behavioural compatibility in the provisioning behaviour of an avian species with biparental care.

3. We recorded parental provisioning behaviour of great tit pairs with known exploratory behaviour. By experimentally cross-fostering offspring, we were able to distinguish between the pre-hatching and rearing effects of parental personality on offspring weight and fledging probability.

4. We show that although all provisioning birds appeared to trade-off visit rate with prey volume, parents with a fast personality type delivered smaller prey for a given visit rate, resulting in a lower overall amount of prey delivered to their offspring. However, this did not result in lighter offspring raised by fast parents. Individual provisioning behaviour did not depend on the combination of personality types within a pair. Moreover, we found pre-hatching effects, instead of the expected rearing effects, of parent personality on offspring success. Females with fastexploring mates laid heavier eggs and offspring with similar biological parents had higher fledging weight in one of the two study years, while foster pairs did not differ in their rearing success depending on their personality or the combination of personalities.

5. Thus, we have shown in this study that despite personality dependent differences in provisioning behaviour, there was no effect of pair compatibility on provisioning performance. Together these results suggest that both direct and indirect benefits of assortative mating for personality may be very context dependent, indicating the potential for fluctuating selection on personality traits and pair compatibility. 


\section{Introduction}

Individuals can gain reproductive benefits by being selective in their choice of mate (Andersson and Iwasa 1996; Andersson and Simmons 2006). While in some cases individuals have the same trait preferences in a potential mate, in other studies individuals have been found to differ in their preferences (Brooks and Endler 2001; Holveck and Riebel 2010; chapter 3). Rather than searching for a universally attractive mate, they look for a compatible mate (Holveck and Riebel 2010; Ihle et al. 2015; chapter 3). In particular, the compatibility of behavioural traits has been shown to be important for mate choice, and to increase reproductive success (Spoon et al. 2006; Schuett et al. 2011; Gabriel and Black 2012; Ouyang et al. 2014). However, what causes higher reproductive success for such pair combinations is not yet clear. One promising candidate for explaining variation between pairs in their rearing success may lie in their provisioning behaviour and the coordination of parental care (Royle et al. 2010). This fitness benefit of behaviourally compatible pairs may work through the compatibility of personalities within a pair.

Recently, an increasing number of studies have focussed on the effect of personality on fitness. In particular, individual differences in personality traits, such as boldness, exploratory behaviour or aggressiveness, have been shown to affect reproductive success, and it is likely that these benefits are mediated through parental care. For instance, aggressive female tree swallows (Tachycineta bicolor) have smaller fledglings (Rosvall 2010). And while bold males have been found to obtain a higher quality territory (song sparrows (Melospiza melodia): Scales et al. 2013), aggressive males are also known to provision less often (Wingfield et al. 1990; House wrens (Troglodytes aedon): Barnett et al. 2012). Therefore, the personality of a mate may have a direct effect on reproductive success and hence may be subject to mate preferences (Schuett et al. 2010).

Additionally, more and more studies indicate that individuals differ in their preferences for a mate depending on their own personality. Indeed, in a number of species mate preferences or choice for behavioural similarity have been reported (Kralj-Fišer et al. 2013; Class et al. 2014). Moreover, behavioural compatibility could increase the likelihood of reproducing, pair coordination (e.g. Raihani et al. 2010; Mariette \& Griffith 2012; Johnstone et al. 2014) and success (Schuett et al. 2011; Ariyomo and Watt 2013; Kralj-Fišer et al. 2013; Class et al. 2014). For instance in cockatiels pairs with a higher behavioural compatibility showed a higher incubation coordination and subsequently hatched more eggs (Spoon et al. 2006) and in zebra finches behaviourally similar pairs were more successful in rearing their offspring (Schuett et al. 2011). 
What explains this variation in rearing success between pairs that differ in behavioural types is not yet clear. One likely candidate is that depending on their behavioural compatibility pairs may differ in their provisioning behaviour and the coordination of parental care. Personality may for instance influence provisioning rules, risk taking, behavioural consistency or the responsiveness to their partners' efforts (Both et al. 2005; Royle et al. 2010; Schuett et al. 2011; Gabriel and Black 2012; Mutzel et al. 2013). Personality-based differences in perception and response to partner foraging effort, consistency and strategy may in turn influence a parent's own investment. When individuals have similar personalities, and thus similar provisioning rules or strategies, this may lead to greater coordination of care, greater investment, and subsequently a higher reproductive success (Royle et al. 2010; Schuett et al. 2011).

Additionally, an individual's own personality may influence its foraging strategy and with this, foraging efficiency and behavioural consistency. Birds of different personality type are known to vary in their parental investment, how they search for prey (Verbeek et al. 1994), and how far they travel to search for prey (van Overveld and Matthysen 2010). Fast explorers for instance have been shown to invest more in nest defence and to behave more aggressively, but they also had a lower feeding rate (Hollander et al. 2008; Mutzel et al. 2013; but see: Barnett et al. 2012). However, when food is scarce, fast explorers are quicker to move to other foraging areas in search for food (van Overveld and Matthysen 2010). Slow explorers on the other hand are expected to be more attentive to environmental cues; they have been found to respond in a more flexibly to changes in food availability (Benus et al. 1987; Verbeek et al. 1994) and are better at exploiting new food sources (Verbeek et al. 1994; Drent and Marchetti 1999), which may be particularly important in conditions with low food availability. Despite these studies finding personality differences in provisioning, a number of other studies found no personality related differences in feeding rate (Patrick and Browning 2011; Schuett et al. 2011). These differences in results may be related to the context dependence of personality effects, with effects depending on the environmental conditions (Dingemanse et al. 2004; Both et al. 2005).

Another reason why personality may affect provisioning rate could be the relation between visit rate and prey volume. Prey volume and search time may trade off if larger prey are more scarce and take longer to find (Werner and Hall 1974; Davies 1977; Grieco 2002; Koo et al. 2016). Therefore, while some individuals may have a lower feeding rate, if they consistently bring in larger prey, the total amount of prey provisioned may still be the same. Different search strategies may lead to different prey types or sizes and different visit rates. Thus, previously found differences in visit related to personality may not necessarily indicate variation in parental investment, but instead suggest differences in 
foraging strategy or consistency. Therefore, instead of focussing on visit rate alone, here we take also prey volume into account.

Although pairs with similar personality have been found to have a higher reproductive success, what exactly causes higher success for these compatible pairs is not yet clear (Schuett et al. 2011; Gabriel and Black 2012). Therefore, the aim of this study was to investigate whether behavioural compatibility could increase reproductive success through provisioning. We first observed mating patterns related to exploratory behaviour, an operational measure of personality in great tits (van Oers and Naguib 2013). Subsequently we studied how provisioning behaviour is related to the individuals' personality, their mates' personality, and their combination. Finally, by cross-fostering chicks between broods, we were able to assess the pre-hatching and rearing effects of parental personality on reproductive success. We expected pairs with similar exploration scores to have higher rearing success rate, due to a greater provisioning effort. 


\section{Methods}

For this study we used wild great tits originating from the field site Boslust, Groot Warnsborn $\left(5^{\circ} 85^{\prime} \mathrm{E}, 52^{\circ} 01^{\prime} \mathrm{N}\right)$, near Arnhem in the Netherlands. Boslust is a mixed wood forest of approximately 70 ha containing 130 nest boxes. The great tit population at the study site is monitored throughout the year. Twice every winter we caught roosting birds during roost inspections of the nest boxes. We took birds that we caught for the first time to the avian indoor facilities at the NIOO-KNAW in Wageningen, the Netherlands, and tested for exploration behaviour in a standardized context as a proxy for personality, using a novel environment test (Verbeek et al., 1994). The next morning, we tested them individually in a novel environment room $(4.0 \times 2.4 \times 2.3 \mathrm{~m})$ with five artificial trees. After birds entered the experimental room, we recorded the number of flights (movements between trees) and hops (movements within trees) within the first $2 \mathrm{~min}$ and used this to calculate an exploration score (Dingemanse et al. 2002). These measures for exploratory behaviour are known to be repeatable $(\mathrm{r}=0.48, \mathrm{P}<0.0001, \mathrm{~N}=111$; Dingemanse et al., 2002) and heritable (Dingemanse et al. 2002; Drent et al., 2003). After testing and weighing all birds were released near their place of capture.

\section{Reproductive success}

During the breeding seasons in 2014 and 2015, we monitored all breeding attempts in the Boslust population. We checked unoccupied nest boxes every five days for initiation of nesting activity. For every nest, we estimated the egg-laying date, start of incubation, and hatching date and recorded the clutch size, brood size, and the number of fledged offspring. We weighed the chicks on the day of hatching (day 0 ) and gave them a down code by selectively removing down tufts to individually identify each chick within a brood. These down codes were visible until at least day 6, after which we gave them a uniquely numbered aluminium ring. On day 1 after hatching we cross fostered the chicks with chicks from two other broods that had hatched on the same day, matched for weight as in Brinkhof et al. (1999) in such a way that each nest contained chicks from two other nests and all chicks were raised by foster parents. In 2014 and 2015, we cross fostered 26 and 48 broods, with 191 and 325 chicks respectively. Chicks were weighed 14 days after hatching (to the nearest $0.01 \mathrm{~g}$ using a digital scale) as a measure of fledging weight, which is closely associated with first year survival (Perrins 1965; Van Balen 1973), and we measured their tarsus length (to the nearest $0.1 \mathrm{~mm}$ using callipers). When the nestlings were 10 days old, we caught the adults at the nest using spring traps. We recorded adult body mass, tarsus length and took a blood sample (approx. $10 \mu \mathrm{l}$ ) from the brachial vein. We fitted unringed adults with an aluminium numbered ring and a plastic leg ring 
containing an RFID tag (IB Technology, Aylesbury, UK). Each nest was checked for chick fledging 21-25 days post-hatching.

\section{Provisioning behaviour}

On days 11 and 12 after hatching, we recorded parental provisioning behaviour, by making both $2 \mathrm{hr}$ video recordings (for detailed provisioning behaviour) and full day RFID recordings. To record visit rates we attached an antenna around the nest box opening that was connected to a RFID reader (Dorset ID, Aalten, The Netherlands). Using this, we recorded all visits of the pair for 1.5 days continuously. From these RFID recordings, we calculated 'working day length' by subtracting the first presumed feeding visit from the last presumed feeding visit. For males, the first feeding visit was assumed to be the first reading of his tag on the day. However, since females sleep in the nest box and will pass the reader when exiting the nest box in the morning, we used the second recording of her RFID tag as the 'first feeding visit'. Using these measures of provisioning day length, we could check whether visit rate was a good representation of provisioning, since some individuals may visit at a lower rate, but for a longer time over the day.

To record prey volume, we also made video recordings using a CCD 420 TV infrared spycamera installed in the lid of the nest box, which was connected to a PV-500L2 portable DVR (LawMate International, Taipei, Taiwan). Videos were analysed to assess parental food provisioning behaviour using Jwatcher Video V1.09 program (http://www.jwatcher.ucla.edu/). All videos were analysed for 2 hours (8:00-10:00 am). We installed camera and PIT recorders the day before data collection, and we analysed the recordings at least 20 minutes after the moment that video and PIT recorders were switched on to make sure the parents had returned to feeding at normal rates.

From the video we recorded the exact time of every visit to calculate the total number of visits, time between visits (the inter-visit interval), and the size of the prey. Apart from measuring the prey length and width on the videos, we also measured the parent's beak width as a reference to estimate the real size of the prey. Beak width has been found to vary relatively little between individuals, so for this study we used a beak width of 5.5 $\mathrm{mm}$ as reference (Lemel 1989). Assuming that the prey had an approximately cylindrical shape we calculated the volume as: $\pi \times 1 / 2$ (prey width) ${ }^{2} x$ prey length.

\section{Extra-pair paternity}

Using the microsatellite data from 17 loci (for more details on microsatellites used see chapter 3), the paternity of chicks was assigned using a likelihood approach in the software program Cervus 3.07 (Marshall et al. 1998). These loci had a combined secondparent exclusion probability (Pre) of 0.9999999945 . We calculated critical values of LOD 
(log likelihood ratio) and delta (difference in LOD scores between the most likely candidate parent and the second most likely candidate parent) using the following parameters in CERVUS: 10000 cycles, $98 \%$ of loci typed, error rate $0.01 \%$, two candidate parents. Offspring were assigned to be extra-pair when these critical values were exceeded in the comparison of the genotypes of the mother, the putative father and the offspring. For 45 offspring in 18 broods ( $9 \%$ of all offspring, in $24 \%$ of all broods), the social father was determined not to be the sire. By comparing these extra-pair offspring with all other males, we were able to identify the extra-pair father for 14 offspring. For more details on genetic analyses see chapter 3 .

\section{Statistical analyses}

\section{Assortative mating}

We explored whether individuals paired non-randomly with regard to personality. To test this, we tested the null hypothesis of random pairing between males and females, by performing a permutation test. In this test we compared the test statistic, that is, the correlation between personality of the male and female of a pair, to a sampling distribution generated by randomly permuting the females and computing the correlation between randomly paired couples (1000 permutations).

\section{Provisioning behaviour}

For all analyses on visit rate or prey volume, we used observations from the 2 hour long video recordings, while for the analyses on day length we used data obtained from the PIT recordings. To analyse how an individual's personality, and the combination with the personality of their mate, influenced provisioning behaviour we used four linear models with visit rate, average prey volume, total prey volume over 2 hours and the length of working day as dependent variables. For all models, we tested the effects of personality, mate personality and sex, and kept brood size (mean \pm SE 5,63 \pm 0.21 )), date and year in the model as control variables. In the model testing effects on working day length, we also controlled for the effects of the real day length at the field site. For the models regarding visit rate and average prey volume we also added prey volume and visit rate respectively, to test for a trade-off between prey volume and visit rate. Additionally, we added the relevant interaction effect between previously mentioned variables. We used backwards elimination based on estimated confidence intervals and the corresponding $\mathrm{P}$ values to obtain the minimal adequate models. Non-significant terms were deleted stepwise, starting with the highest order interactions and/or the least significant term $(\mathrm{P}<0.05)$. However, since the provisioning behaviour of each individual may appear in the analysis twice, once as the dependent variable and again as an independent variable explaining 
partner provisioning behaviour, a potential pseudo-replication may arise. Therefore, we randomly selected one parent for each nest, so that for half of the nests female provisioning was the dependent variable and the male provisioning behaviour was the independent variable, while for the other half the reverse was true (see Hinde \& Kilner 2007). This random selection of one parent from each nest as the dependent variable was repeated and for each analysis we present the average results from 1000 iterations. All continuous variables were standardized as Z-scores before analysis. Since backwards elimination may be susceptible to the order in which variables are eliminated from the models, we compared the results from the backwards elimination with the results using an information criterion model selection approach (Appendix B), which produced qualitatively the same results. For all analyses on provisioning behaviour we used the packages lme4 (version 1.1-12; Bates et al. 2015) and MuMln (version 1.40.0; Bartón 2017) in $R$ software system (version 3.4.1; R Development Core Team 2017).

\section{Reproductive success}

We tested the effects of parent personality on fledging weight (weight of the offspring when 14 days old), for both biological and foster parents. For this we ran a linear mixed model with biological brood and foster brood as random effects to account for the crossfoster design and the multiple chicks per brood. As explanatory variables, we added the personality of the biological and foster parent pairs. For the effects of biological fathers and relatedness, we used the genetic sire, which in some cases was the extra-pair sire. We also tested for an interaction effect of parental personality on reproductive success. Because brood size and offspring sex (as categorical variable) are known to affect chick weight we also controlled for these. We also added brood size, catch time, catch date and year (as a categorical variable) and the interaction between catch date and year (hatch date * year) as control variables. Since egg weight is known to influence offspring growth and fledging probability we also added this variable as a control variable to the models testing foster parent effects. We started with the full model and used stepwise backward selection to obtain the minimal adequate models.

We tested for differences in fledging probability for each offspring with a binary generalized linear mixed model, with fledging (yes or no) as a dependent variable and the personality of biological and foster parents as explanatory variables. We also tested for an interaction effect of parental personality scores. We kept brood size, hatch date, offspring sex and year in the model as control variables and the interaction between catch date and year (hatch date * year) as control variables (offspring sex and year as a categorical variables). Biological brood and foster brood were random effects to account for the crossfoster design. 
Finally, we tested for the effects of parental provisioning on offspring weight and fledging using a linear mixed model and a binary generalized linear mixed model respectively. Using backwards elimination, we tested the effects of visit rate and average prey volume on offspring fledging weight and fledging probability. Additionally, we tested the effect of total prey volume on offspring fledging weight and fledging probability. All models contained brood size, sex, date and year as control variables, and the model on fledging weight also contained weighing time as a control variable.

Similar to the models on provisioning, the models on reproductive success may also be susceptible to the order in which variables are eliminated when using backwards elimination procedures. Therefore, we also compared these model outcomes to additional models using an information criterion model selection approach (Appendix B). The results of both analyses were qualitatively the same except for the analysis on egg weight which was not significant in the model selection approach. Therefore, we treat this result with caution in our interpretation. For all analyses on reproductive success we used the packages lme4 (version 1.1-12; Bates et al. 2015) and MuMln (version 1.40.0; Bartón 2017) in R software system (version 3.4.1; R Development Core Team 2017). 


\section{Results}

\section{Mate choice}

Mate choice patterns on the basis of personality did not deviate from random mating; the personality types of pairs were not more correlated than a randomly generated distribution of pairs drawn from the population (random correlation 95\% confidence interval $=[-0.29,0.37]$; correlation of breeding pairs $=0.30, \mathrm{~N}=35 ; 10000$ permutations).

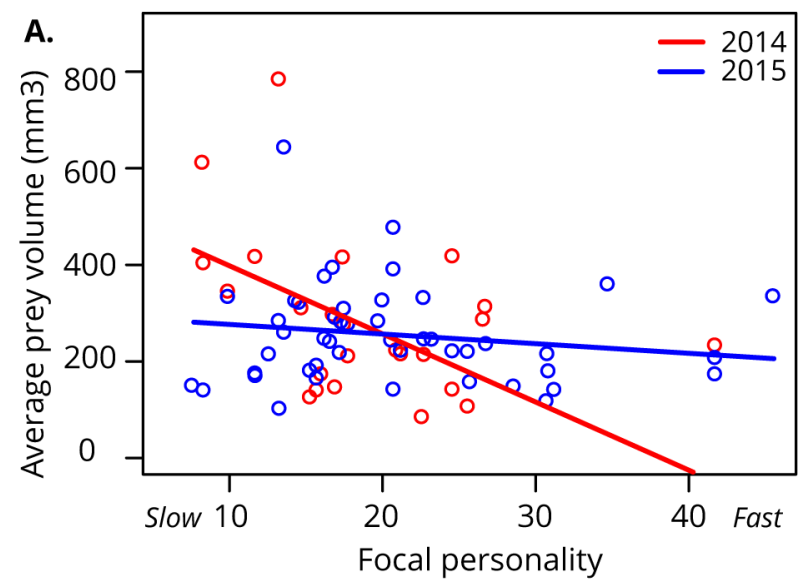

\section{Provisioning}

\section{Prey volume}

The average prey volume birds provisioned to their offspring, was associated with their personality depending on the year, while controlling for visit rate (Figure 5.1a; personality * year: 95\% CI= [18.68, 215.34], $\mathrm{P}=0.03$, Table 1). In 2014, faster birds brought in smaller prey, while in 2015 personality types did not differ in prey volume delivered. Furthermore, birds mated with faster birds tended to bring in smaller prey (Figure 5.1b; mate personality: 95\% CI= [96.23, -4.75], $\mathrm{P}=0.06$, Table 1). Prey volume is strongly associated with the visit rate: birds that bring in large prey items on average also visit less often (visit rate: $95 \% \mathrm{CI}=[-113.59$, 27.99], $\mathrm{P}=0.002$, Table 1).

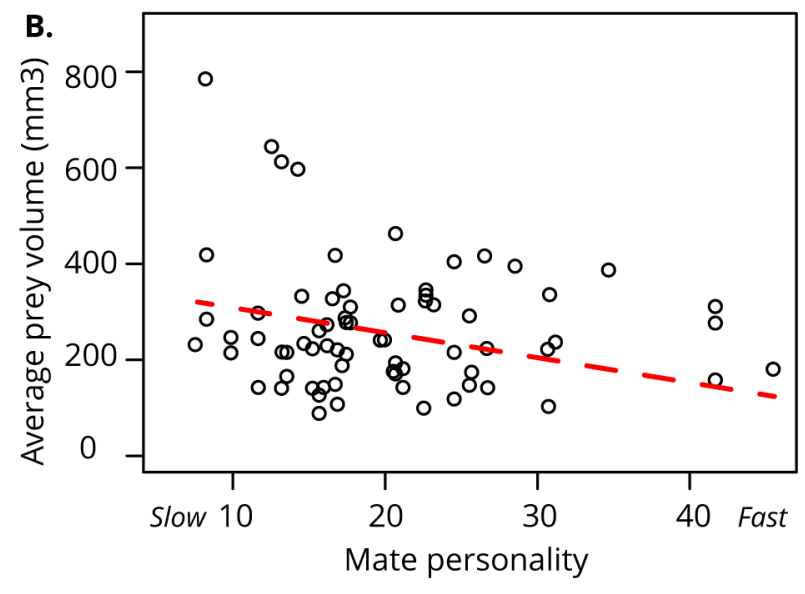

Figure 5.1 - The relation between average volume of prey items delivered and personality and mate personality over the 2 hrs measured. Circles represent actual data points and lines represent model outcome. A) There was a negative relationship between personality and average prey volume depending on the year (while controlling for total number of feeding visits). B) Birds with faster mates brought in on average smaller prey items (while controlling for total number of feeding visits). 
Table 1: Average prey volume. All variables tested in the linear mixed model with average prey volume (over the two observed hours) as the dependent variable (number focal parents $=54$, broods $=27$ ). Given is the mean estimate ( $\beta$ ), 95\% confidence interval of the estimate ( $95 \%$ CI for $\beta$ ) and the significance ( $P$ value). Significant terms $(P<0.05)$ are indicated in bold, and trends $(P=0.05-0.10)$ are indicated in italics.

\begin{tabular}{lccc}
\hline & $\boldsymbol{\beta}$ & $\mathbf{9 5 \%}$ CI for $\boldsymbol{\beta}$ & P value \\
\hline Minimal adequate model & & & \\
Intercept & 254.86 & - & - \\
Brood size & 2.35 & {$[-34.9,45.33]$} & 0.91 \\
Date & -53.70 & {$[-84.44,-27.41]$} & $<0.001$ \\
Year & 2.03 & {$[-56.67,62.39]$} & 0.95 \\
Visit rate & -67.62 & {$[-113.59,-27.99]$} & $\mathbf{0 . 0 0 2}$ \\
Personality & -117.97 & {$[-221.16,-69.81]$} & 0.004 \\
Mate personality & -43.17 & {$[-96.23,-4.75]$} & 0.06 \\
Date* year & 68.94 & {$[24.89,114.6]$} & $\mathbf{0 . 0 1}$ \\
Personality * year & 101.69 & {$[18.68,215.34]$} & $\mathbf{0 . 0 3}$ \\
\hline Dropped terms & & & \\
Sex & 33.94 & {$[-45.83,121.65]$} & 0.43 \\
Personality * sex & 20.51 & {$[-86.47,110.74]$} & 0.69 \\
Personality * visit rate & 1.42 & {$[-1.31,4.8]$} & 0.38 \\
Personality * mate personality & 18.45 & {$[-25.35,64.53]$} & 0.43 \\
Mate personality * sex & 2.40 & {$[-97.13,122.05]$} & 0.97 \\
Mate personality * year & 65.90 & {$[-30.96,202.27]$} & 0.28 \\
\hline
\end{tabular}




\section{Visit rate}

When controlling for prey volume, fast exploring birds had a lower visit rate than slow birds (Figure 5.2a; personality: 95\% CI= [-6.82, -1.15], $\mathrm{P}=0.01$, Table 2) Birds that brought on average larger prey items had a lower visit rate (Figure 5.2b; average prey volume: 95\% CI= [-7.49, -2.28], $\mathrm{P}=0.0003$, Table 2 ). The number of visits did not vary over different personality combinations within pairs (personality * mate personality: $95 \% \mathrm{CI}=$ [-4.04 \pm 1.79$], P=0.44$, Table 2). In either model on prey volume or visit rate, when taking out visit rate or prey volume respectively, personality became non-significant, indicating that, on average, birds of different personalities did not differ in their visit rate nor in their average prey volume. Only by controlling for this trade-off we found that fast birds had lower visit rates for a certain prey volume (and the other way around, delivered a lower prey volume for a certain visit rate; Figure 5.2c).
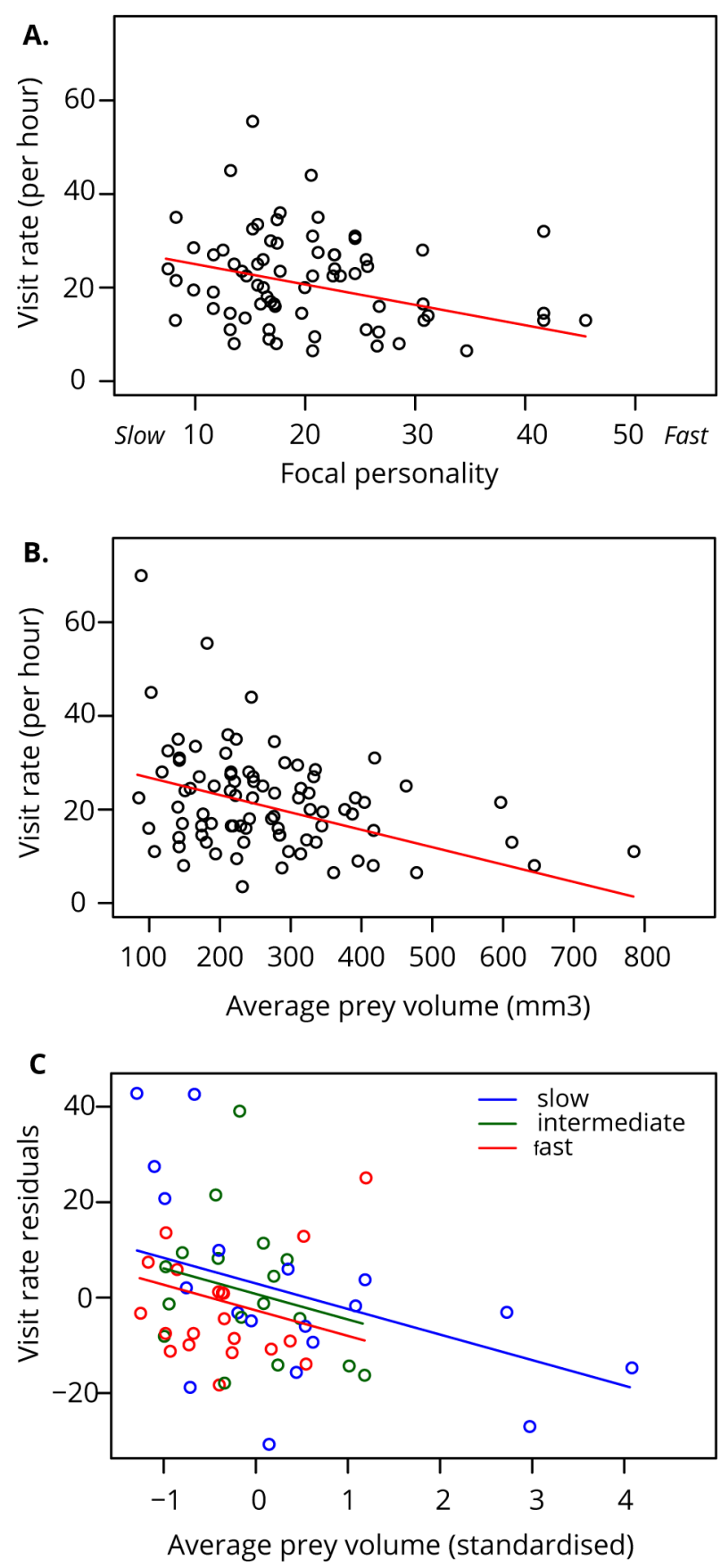

Figure 5.2 - Relation between personality and prey volume and visit rate. Circles represent actual data points and lines represent model outcome. A) Faster birds had a lower visit rate (while controlling for prey volume). B) Birds that brought in large prey on average had a lower visit rate. C) Graph visualising residuals from the model testing effects on visit rate plotted against average prey volume. Models contained exploration score as continuous variable, here for visualisation purposes we indicate fast, intermediate and slow 
Table 2: Visit rate. All variables tested in a linear mixed model with visit rate (visits per hour) as the dependent variable (Number focal parents $=54$, broods $=27$ ). Given is the mean estimate $(\beta), 95 \%$ confidence interval of the estimate ( $95 \%$ CI for $\beta)$ and the significance (P value). Significant terms $(P<0.05)$ are indicated in bold, and trends $(P=0.05-0.10)$ are indicated in italics.

\begin{tabular}{lccc}
\hline & $\boldsymbol{\beta}$ & $\mathbf{9 5 \%}$ CI for $\boldsymbol{\beta}$ & P value \\
\hline Minimal adequate model & & & \\
Intercept & 19.76 & - & - \\
Brood size & -2.22 & {$[-\mathbf{4 . 3}, \mathbf{- 0 . 3 1 ]}$} & 0.04 \\
Date & 3.83 & {$[\mathbf{0 . 1}, \mathbf{7 . 7 5}]$} & 0.05 \\
Year & 1.70 & {$[-0.5,3.77]$} & 0.13 \\
Personality & -3.58 & {$[-6.82, \mathbf{- 1 . 1 5}]$} & $\mathbf{0 . 0 1}$ \\
Prey volume & -4.71 & {$[-\mathbf{7 . 4 9}, \mathbf{- 2 . 2 8}]$} & $\mathbf{0 . 0 0 0 3}$ \\
Date ${ }^{\text {year }}$ & 3.69 & {$[\mathbf{1 . 2 1}, \mathbf{6 . 1 9 ]}$} & $\mathbf{0 . 0 0 5}$ \\
\hline Dropped terms & & & \\
Mate personality & -1.89 & {$[-4.94,1.63]$} & 0.25 \\
Sex & 3.01 & {$[-3.57,9.53]$} & 0.37 \\
Personality * sex & 3.82 & {$[-1.06,10.31]$} & 0.18 \\
Personality * year & 2.66 & {$[-3.89,10.97]$} & 0.48 \\
Personality * prey volume & 1.79 & {$[-2.7,6.11]$} & 0.40 \\
Personality * mate personality & -1.18 & {$[-4.04,1.79]$} & 0.44 \\
Mate personality * sex & 1.61 & {$[-5.53,9.51]$} & 0.66 \\
Mate personality * year & 3.43 & {$[-3.24,13.15]$} & 0.43 \\
\hline
\end{tabular}

\section{Total prey volume}

Fast birds provisioned a lower total prey volume to their offspring over 2 hours than slow birds (Figure 5.3; personality: 95\% CI= [-3386.76, 793.84], $\mathrm{P}=0.01$, Table 3). This association did not depend on their mates' personality (personality * mate personality: 95\% CI= [-1565.82, 1343.54], $\mathrm{P}=0.79$, Table 3).

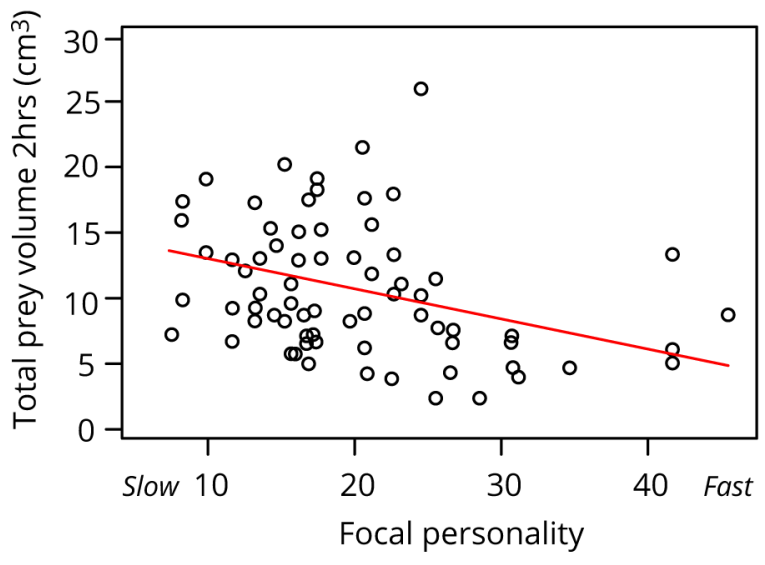

Figure 5.3 - Effects on total prey volume brought in during parental feeding visits over $2 \mathrm{hrs}$. Circles represent actual data points and the line represents the model outcome. 
Table 3: Total prey volume. All variables tested in the linear mixed model with the total prey volume (over the two observed hours) as the dependent variable (number focal parents $=54$, broods $=27$ ). Given is the mean estimate ( $\beta$ ), 95\% confidence interval of the estimate (95\% CI for $\beta$ ) and the significance ( $P$ value). Significant terms $(P<0.05)$ are indicated in bold, and trends $(P=0.05-0.10)$ are indicated in italics.

\begin{tabular}{lccc}
\hline & $\boldsymbol{\beta}$ & $\mathbf{9 5 \%} \mathbf{C I}$ for $\boldsymbol{\beta}$ & P value \\
\hline Minimal adequate model & & & \\
Intercept & 10547.83 & - & - \\
Brood size & 590.01 & {$[-488.34,1548.94]$} & 0.26 \\
Date & -304.83 & {$[-1083.85,545.36]$} & 0.47 \\
Year & 575.54 & {$[-1429.87,2703.91]$} & 0.59 \\
Personality & -1892.76 & {$[-3386.76,-793.84]$} & $\mathbf{0 . 0 1}$ \\
\hline Dropped terms & & & \\
Mate personality & -1453.47 & {$[-3713.73,701.43]$} & 0.19 \\
Sex & 2278.56 & {$[-1303.81,5812.88]$} & 0.22 \\
Date * year & -1518.66 & {$[-3730.87,574.51]$} & 0.17 \\
Personality * sex & 708.75 & {$[-2165.97,3894.17]$} & 0.65 \\
Personality * year & 2248.82 & {$[-1320.86,6882.88]$} & 0.30 \\
Personality * mate personality & -209.34 & {$[-1565.82,1343.54]$} & 0.79 \\
Mate personality * sex & 405.88 & {$[-3244.72,4769.37]$} & 0.84 \\
Mate personality * year & 2270.08 & {$[-2088.37,8404.53]$} & 0.43 \\
\hline
\end{tabular}

\section{Working day length}

Females feeding visits commonly started earlier and ended later, resulting in longer 'working day lengths' compared to males (Figure 5.4; sex, 95\% CI= [-0.83, -0.05], $\mathrm{P}=0.03$, Appendix A Table S1). Birds of different personality did not vary in their day length (personality: $95 \% \mathrm{CI}=[-0.12,0.13], \mathrm{P}=0.74$. Moreover, visit rate was not associated with working day length (visit rate: $95 \% \mathrm{CI}=$ $[-0.05,0.4], \mathrm{P}=0.20$, Table S1), suggesting that birds did not compensate for a lower provisioning rate by making longer working days. This indicates that visit rate is a good representation of parental provisioning effort.

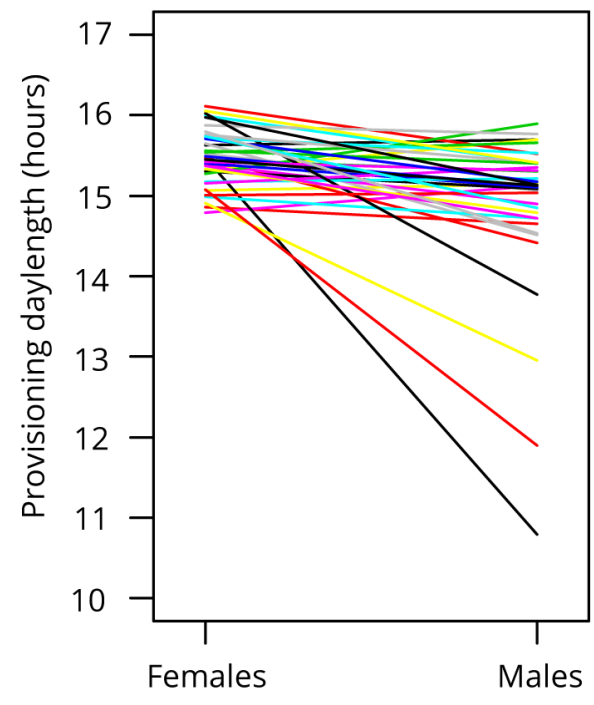

Figure 5.4 - Female feeding visits commonly started earlier and ended later, resulting in longer working day lengths than males. Figure shows actual data with each line representing a unique brood. 


\section{Reproductive success}

\section{Fitness effects of personality and mate personality}

While there were no effects of parental personality on lay date (female personality, $\mathrm{LM}: \mathrm{t}_{3,53}=0.79, \quad \mathrm{P}=0.45$; male personality, LM: $\mathrm{t}_{4,32}=0.13$, $\mathrm{P}=0.89$, Table S2) and clutch size

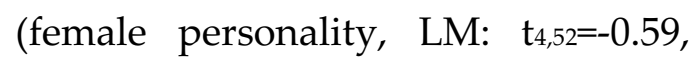
$\mathrm{P}=0.56$; male personality, $\mathrm{LM}: \mathrm{t}_{6,30}=-$ 0.06, $\mathrm{P}=0.96$, Table S3), females paired with faster males laid heavier eggs (Figure 5.5: male personality, LM: $\mathrm{t}_{5,41}=2.07, \mathrm{P}=0.04$, Table S4).

The weight at day 14 of chicks was not

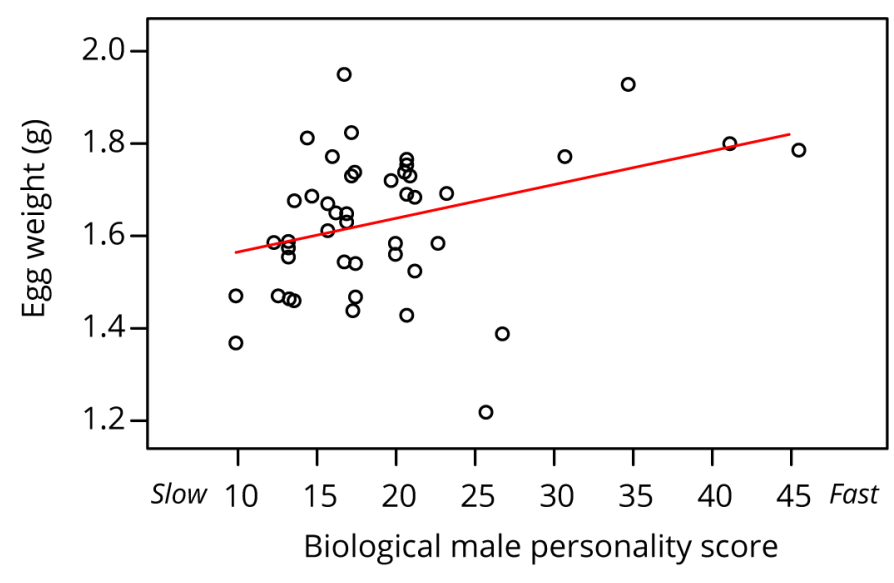

Figure 5.5 - Females with faster males laid heavier eggs on average. Circles represent actual data points and lines represent model outcomes. associated with foster parent personality nor the combination of foster parent personalities (female personality, LMM: $\mathrm{t}_{2.69}=0.57, \mathrm{P}=0.58$; male personality, $\mathrm{LMM}$ : $\mathrm{t}_{31.31}=1.70, \mathrm{P}=0.10$, female personality * male

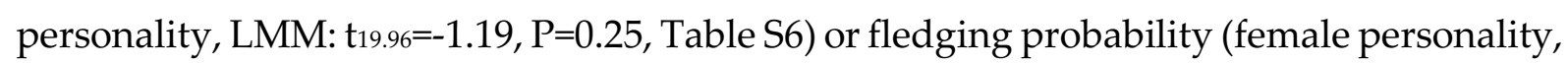
GLMM: $Z=0.07, P=0.94$; male personality, GLMM: $Z=-0.99, P=0.32$, female personality * male personality, GLMM: $Z=-1.12, P=0.26$, Table $S 8$ ).

Fledging weight was associated with the combination of the biological parents' exploratory scores (Figure 5.6; biological female personality * biological male personality * year: $\mathrm{t} 36.35=-2.49, \mathrm{P}=0.02$, Table S5). In 2014, offspring produced by assortative pairs had a higher fledging weight, while in 2015 there was no such effect. However, offspring fledging probability was not associated with the biological parents' personality (female personality, GLMM: $\mathrm{Z}=-0.02$, $\mathrm{P}=0.98$; male personality, GLMM: $\mathrm{Z}=0.48, \mathrm{P}=0.63$, female personality * male personality, GLMM: $\mathrm{Z}=1.08, \mathrm{P}=0.28$, Table S7). 


\section{Fitness effects of provisioning}

A higher total prey volume delivered to the brood did not increase offspring weight (total prey volume brood, LMM: t33.25=0.07, P=0.94, Table S10) or fledging probability (total prey volume brood, GLMM: $\mathrm{Z}=0.22$, $\mathrm{P}=0.83$, Table S12), when controlling for brood size. Instead, broods that received a lower average prey volume tended to have a higher fledging weight (average prey volume, LMM: $\mathrm{t}_{33.7}=-1.82$, $\mathrm{P}=0.08$, Table S9). Broods that received more feeding visits had a higher fledging probability (visit rate, GLMM: $Z=2.56, P=0.02$, Table S11).
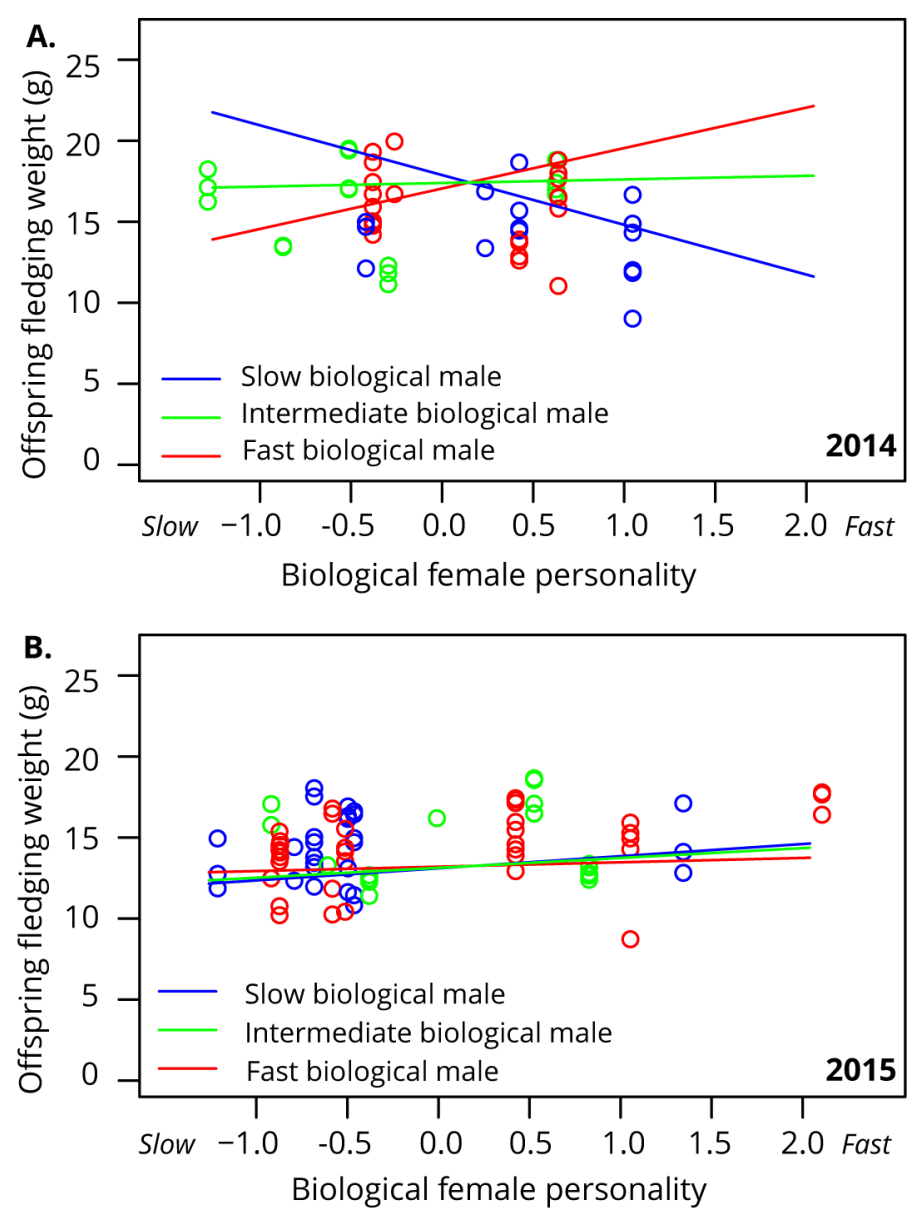

Figure 5.6 - Parental personality and offspring fledging weight. In 2014 the offspring produced by assortative pairs had a higher fledging weight $(A)$, while in 2015 there was no effect of biological parents' personality (B). Colours indicate biological male's personality types. Models contained male personality, exploration score, as continuous variable, here for visualisation purposes we indicate fast, intermediate and slow explorers with different colours (lines represent average of fastest, slowest and intermediate $33.3 \%$ of the birds). Circles represent actual data points and lines represent model outcome. 


\section{Discussion}

Here we show that while birds of different personality type varied in their provisioning behaviour, their parental investment did not depend on their partners' personality. After controlling for prey volume, faster birds made fewer feeding visits to the nest box, and when controlling for visit rate, faster birds brought in smaller prey. These differences in provisioning strategy led to faster explorers bringing a lower total prey volume to their offspring. In contrast to other studies, we did not find that assortative pairs were better parents; similar pairs were not more successful in rearing their offspring, nor did we find other personality related differences in reproductive success. This may be related to the fact that reproductive success was higher for pairs that had a high visit rate (and, correlated, a low average prey volume) rather than a high total prey volume. Instead of the expected rearing effects, we found pre-hatching effects on offspring. Females with faster mates tended to lay heavier eggs and, in one of the two years, original parents that mated assortatively for personality had a reproductive benefit in terms of offspring fledging weight. This suggests that compatibility for these behavioural traits may have a pre-hatching benefit for offspring in some years, but not in others.

A number of studies have reported fitness benefits for pairs that mated assortatively for personality (Budaev et al. 1999; Sinn et al. 2006; Schuett et al. 2011; Ariyomo and Watt 2013; Class et al. 2014). Although these assortative mating patterns for personality have been found in wild populations (Groothuis and Carere 2005; Gabriel and Black 2012), quite often significant patterns are absent or fluctuate between years (Dingemanse 2004). Despite the fact that reproductive benefits for assortative pairs have also been reported for great tits (Dingemanse et al. 2004; Both et al. 2005; David et al. 2015), assortative mating patterns for personality have not, with one study actually finding disassortative mating patterns (Dingemanse et al. 2004). There are different potential explanations for this discrepancy. Differences in the occurrence and stability of assortative mating patterns between populations may be correlated with the extent to which the benefits of assortative mating are either stable or context dependent. In Steller's jays (Cyanocitta stelleri), similarity in personality traits increased reproductive success over all years measured and assortative mating patterns were found consistently for all years. However, in great tits, the effects of personality and behavioural compatibility on fitness and reproductive success fluctuate between years (Dingemanse et al. 2004; Both et al. 2005; Nicolaus et al. 2016), which could be an explanation for fluctuations in the strength and direction of choice patterns. Another explanation may be that in great tits, other factors, such as territory quality, availability of potential mates or female-female interactions (Drent 1983) 
influence mating patterns, which may explain the absence of assortative mating patterns, despite the potential fitness benefits.

It has been suggested that the higher reproductive success for assortative pairs works through a higher behavioural compatibility resulting in greater coordination and less parental conflict, and possibly a higher provisioning rate (Royle et al. 2010; Schuett et al. 2011). In this study, we did not find such a difference in provisioning performance depending on the combination of personalities within a pair. Rather than affecting the way they provisioned depending on their partners' personality, in our study personality was mainly related to an individuals' own provisioning strategy and performance. As previously noted in great tit and blue tits (Grieco 2001; Grieco 2002; Hinde 2005), we found a negative correlation between prey volume and visit rate, emphasizing the importance of considering prey volume when measuring parental provisioning. When taking into account this trade-off in our analyses, faster explorers were found to provision less, having a lower visit rate for a given prey volume and vice versa. Fast explorers have previously been found to be more aggressive (Verbeek et al. 1996), which in turn has been found to be related to an increase in nest defence and a decrease in parental feeding visits (McGlothlin et al. 2007; Hollander et al. 2008; Mutzel et al. 2013). However since birds may use different search strategies depending on their personality (Verbeek et al. 1994; van Overveld and Matthysen 2010), it is possible that these fast explorers may have been able to compensate for these differences in visit rate by bringing in larger prey items. Here we found however, that when controlling for the trade-off between prey volume and visit rate, fast explorers indeed brought in a lower total amount of prey. There may be different explanations for this. Firstly it is possible that fast explorers simply are slower at finding prey, because they are travelling further, more selective in prey choice, or simply less skilled at finding prey (Verbeek et al. 1994; van Overveld and Matthysen 2013). Alternatively, it is possible that fast explorers spend more time on other aspects of parental investment, such as territory or nest defence. In that case, however, a relation with sex would have been expected (Mutzel et al. 2013) since in great tits it is predominantly males that defend territories and not females. Moreover, personality may be correlated with territory choice and quality (Both et al. 2005), and factors such as environmental availability of certain prey types may also play a role in the correlation between provisioning behaviour and personality. This might also explain the correlation between average prey volume individuals brought to their offspring and their mate's personality. Thus, in what exact way birds of different personality type differed in their provisioning behaviour cannot be concluded from this data. Further experiments and observations on 
personality related differences in provisioning strategies, techniques and performance and would be able to give a better understanding of why individuals differ in provisioning.

Despite a lower provisioning performance by fast explorers, there was no relation between personality and rearing success. A potential explanation may lie in the relation between provisioning and reproductive success: a higher total prey volume did not directly predict higher offspring weight or fledging probability. Instead, a high visit rate and (correlated) low average prey volume were related to reproductive success. This suggests that the ratio between visit rates and prey volumes is more important than the absolute amount of prey brought in. Larger prey may have a lower nutritional value (Naef-Daenzer et al. 2000), while prey volumes and feeding intervals may affect offspring digestive efficiency (Budden and Wright 2008; Grodzinski et al. 2009). Alternatively fast and slow individuals may differ in the prey type they predominantly forage on (Costantini et al. 2005; Chang et al. 2017). While prey sizes may on average be the same, if fast explorers predate on more nutritious prey and deliver these to their offspring, this may have counteracted the lower total prey volume they brought in.

Additionally, similar to provisioning, the combination of parental personalities was not related to their reproductive success. The personality of the pair mates and the combination of personalities did not correlate with their reproductive success through rearing effects. The beneficial effects of assortative mating and behavioural compatibility may depend on the environmental conditions causing between-year differences in the benefits of mating assortatively, with the reproductive benefits of mating assortatively differing between years with high or low food availability (Dingemanse et al. 2004). Another explanation for the higher reproductive success found in other studies, might lie in how assortative and disassortative pairs cope with challenges. For instance, in great tits, after an experimental brood size enlargement which suddenly increased the broods' food demand, assortative pairs increased their visit rate more than disassortative pairs (David and Cézilly 2011). In eastern bluebirds (Sialia sialia), assortative mating had a reproductive benefit in habitats with high interspecific competition, but not in areas with low competition (Harris and Siefferman 2014). Thus, although our results suggest the absence of personality related differences in responsiveness, since we only measured baseline provisioning in two subsequent years, we might not have picked up on these differences between pairs. Measuring these effects over multiple years and correlating them with the environmental variables or experiments in which environmental conditions are manipulated may give a better insight into how selection pressures may favour certain combinations of personalities in a pair under some circumstances and other combinations under other circumstances. 
Against our expectations we found that despite the absence of direct effects of personality on reproductive success, personality had a pre-hatching effect on reproductive success. Females with faster males laid heavier eggs. However, since this effect was not found to be significant when analysing using a model selection approach, this result has to be interpreted with some caution. This tendency of females to lay heavier eggs could a form of compensatory investment, where females might compensate for their fast males who provision less (Hollander et al. 2008; Mutzel et al. 2013). However, since fast females also provision less, a compensation in egg weight could also be expected for fast females, but this was not found by this study. Alternatively, assuming that fast males hold better territories (Both et al. 2005; Scales et al. 2013) females could also invest extra in their eggs when they are paired with high quality males. Another striking result was the higher fledging weight of offspring (which is correlated with post fledging survival, Van Balen 1973), from assortative biological parents in 2014, while there was no effect on offspring weight in 2015. Since personality is known to have a heritable component, offspring from extreme and similar pairs is more likely to have extreme personalities but will also have a lower genetic variation on these genes. In a study on great tits (van Oers et al. 2008), assortative pairs had a higher rate of extra pair paternity, which suggests that in this way females could increase the genetic variation in their offspring, or could lead to more intermediate offspring in terms of personality. In our case, offspring from similar pairs had a benefit in one year but not in another, indicating that not just rearing effects, but also the pre-hatching effects of personality, may be context dependent. Potentially, offspring with extreme personalities, coming from assortative parents, had a competitive advantage over more intermediate foster siblings, perhaps through different, but equally successful strategies, giving them a higher fledging weight. Alternatively, it is possible that this effect on offspring weight works through maternal hormone investment in the eggs. Maternal androgens in eggs are known to affect offspring early growth and survival, although these effects may depend on environmental conditions (Hinde et al. 2009; Von Engelhardt and Groothuis 2011), and the level of androgen deposit has been found to correlate with female personality and her mates' personality (Ruuskanen et al. 2017). Eggs from disassortative pairs differed in their androgen levels from assortative pairs (Ruuskanen et al. 2017). Such different androgen levels deposited by the biological females of assortative pairs compared to disassortative pairs could potentially explain the observed weight differences in their offspring and the context dependence of this effect.

To conclude, in this study we showed that although personality affects provisioning behaviour, assortative mating did not lead to an increased provisioning performance, nor did it increase reproductive success. We did however find indirect effects of male 
personality on egg weight, and effects of pair compatibility on chick weight. In view of previously found benefits of personality similarity on fitness, these results suggest that both direct and indirect benefits of assortative mating for personality may be very context dependent, which indicates fluctuating selection on personality traits and pair compatibility, with a potential for fluctuating selection on preferences for mate personality.

\section{Acknowledgements}

We are grateful to Maxime Kole, Lisanne van den Bos, Antoine Juigner, Kristin Sauerland, Neeltje Hermus and Coen van Tuijl for their help with collecting the data analysed here. Many thanks to Piet de Goede, Marylou Aaldering, Ruben de Wit, Coretta Jongeling and Anouk de Plaa for their help with taking care of the birds. We also thank Stichting Geldersch Landschap en Kasteelen for permission to do fieldwork in the Boslust forest. LZ was funded by a WIAS PhD scholarship from Wageningen University and Research. This research was supported by 'SEX SEL-VAR' project number 334544 funded by a Marie Curie Career Integration Grant from the European Commission (FP7) awarded to CAH, by the Dobberke foundation for comparative psychology and by the Royal Netherlands Academy of Arts and Sciences. Permission for animal experiments was granted by the Animal Ethics Committee of the KNAW (DEC-KNAW) under protocol number NIOO13.12 to KVO and LZ.

\section{Author contributions}

LZ, CAH and KVO designed the study, LZ and PC collected the data, LZ and PC analysed the data and LZ drafted the initial version of the manuscript. All authors contributed critically to the drafts and gave final approval for publicat 
Supplement to chapter 5 


\section{Appendix A - Model tables}

Table S1: Working day length. Table consists of all variables tested in the linear mixed model with the working day length (time between first and last feeding visit) as the dependent variable ( $N=54$, broods $=27)$. Given is the mean estimate ( $\beta), 95 \%$ confidence interval of the estimate ( $95 \%$ CI for $\beta$ ) and the significance (P value). Significant terms $(P<0.05)$ are indicated in bold, and trends $(P=0.05-0.10)$ are indicated in italics.

\begin{tabular}{lccc}
\hline & $\boldsymbol{\beta}$ & $\mathbf{9 5 \%} \mathbf{C I}$ for $\boldsymbol{\beta}$ & P value \\
\hline Minimal adequate model & & & \\
Intercept & 15.55 & - & - \\
Brood size & 0.04 & $-0.09,0.16$ & 0.57 \\
Date & -0.24 & $-0.74,0.24$ & 0.34 \\
Year & -0.13 & $-0.67,0.41$ & 0.65 \\
Sex & -0.57 & $-0.92,-0.21$ & $\mathbf{0 . 0 0 2}$ \\
Real day length & 0.27 & $-0.2,0.74$ & 0.27 \\
\hline Dropped terms & & & \\
Visit rate & 0.15 & $-0.05,0.4$ & 0.20 \\
Personality & 0.08 & $-0.03,0.19$ & 0.19 \\
Mate personality & 0.07 & $-0.19,0.35$ & 0.61 \\
Personality * sex & -0.10 & $-0.44,0.25$ & 0.56 \\
Personality * year & 0.32 & $0.06,0.81$ & 0.09 \\
Personality * all visits & 0.03 & $-0.19,0.27$ & 0.77 \\
Personality ${ }^{*}$ mate personality & -0.02 & $-0.17,0.1$ & 0.74 \\
Mate personality * sex & -0.03 & $-0.43,0.41$ & 0.90 \\
Mate personality * year & 0.33 & $-0.18,1.09$ & 0.30 \\
\hline
\end{tabular}


Table S2: Lay date. Table consists of all variables tested in the linear regression with the lay date (days after $1^{\text {st }}$ of April) as the dependent variable $(N=37)$. Given is the estimate, test statistic ( $t$ value) and the significance $(P$ value). Significant terms $(P<0.05)$ are indicated in bold, and trends $(P=0.05-0.10)$ are indicated in italics.

\begin{tabular}{lccc}
\hline & Estimate & $\mathbf{t}$ value & P value \\
\hline Minimal adequate model & & & \\
Intercept & 20.64 & - & - \\
Year & 6.17 & 4.21 & $<\mathbf{0 . 0 0 0 1}$ \\
Clutch size & -1.10 & -1.97 & $\mathbf{0 . 0 5}$ \\
\hline Dropped terms & & & \\
Bio female personality * Bio male personality * year & 0.00 & 0.01 & 0.99 \\
Bio male personality * year & 0.10 & 0.20 & 0.84 \\
Bio female personality * Bio male personality & 0.01 & 0.31 & 0.76 \\
Bio female personality * year & -0.13 & -0.52 & 0.60 \\
Bio male personality & 0.02 & 0.13 & 0.89 \\
Bio female personality & 0.06 & 0.79 & 0.44 \\
\hline
\end{tabular}

Table S3: Clutch size. Table consists of all variables tested in the linear regression with the clutch size as the dependent variable $(N=37)$. Given is the estimate, test statistic ( $t$ value) and the significance ( $P$ value). Significant terms $(P<0.05)$ are indicated in bold, and trends $(P=0.05-0.10)$ are indicated in italics.

\begin{tabular}{lccc}
\hline & Estimate & $\mathbf{t}$ value & P value \\
\hline Minimal adequate model & & & \\
Intercept & 8.38 & - & - \\
Year & -0.77 & -2.84 & $\mathbf{0 . 0 1}$ \\
Lay date & -0.10 & -0.44 & 0.66 \\
Year * lay date & -0.29 & -1.06 & 0.29 \\
\hline Dropped terms & & & \\
Bio female personality * Bio male personality * year & 0.00 & -0.15 & 0.88 \\
Bio female personality * Bio male personality & 0.00 & 0.16 & 0.87 \\
Bio male personality * year & -0.03 & -0.28 & 0.78 \\
Bio male personality & 0.00 & -0.06 & 0.95 \\
Bio female personality * year & -0.02 & -0.70 & 0.48 \\
Bio female personality & -0.01 & -0.59 & 0.56 \\
\hline
\end{tabular}


Table S4: Egg weight. Table consists of all variables tested in the linear regression with the mean egg weight as the dependent variable $(N=34)$. Given is the estimate, test statistic ( $t$ value) and the significance ( $P$ value). Significant terms $(P<0.05)$ are indicated in bold, and trends $(P=0.05-0.10)$ are indicated in italics.

\begin{tabular}{lccc}
\hline & Estimate & $\mathbf{t}$ value & P value \\
\hline Minimal adequate model & & & \\
Intercept & 1.52 & - & - \\
Bio male personality & $\mathbf{0 . 0 4}$ & $\mathbf{2 . 0 7}$ & $\mathbf{0 . 0 4}$ \\
Year & -0.01 & -0.22 & 0.83 \\
Clutch size & 0.02 & 0.90 & 0.37 \\
Lay date & 0.05 & 1.45 & 0.15 \\
Year ${ }^{*}$ lay date & -0.05 & -1.24 & 0.22 \\
\hline Dropped terms & & & \\
Bio female personality * Bio male personality * year & 0.00 & -0.81 & 0.43 \\
Bio male personality * year & 0.00 & -0.15 & 0.88 \\
Bio female personality * year & 0.01 & 0.71 & 0.48 \\
Bio female personality * Bio male personality & 0.00 & -0.72 & 0.48 \\
Bio female personality & 0.00 & -0.51 & 0.61 \\
\hline
\end{tabular}

Table S5: Offspring fledging weight biological parents. Table consists of all variables tested in the linear mixed model with the offspring weight on at 14 days old as the dependent variable ( $N$ offspring $=136$, broods =33). Given is the estimate, degrees of freedom ( $d f)$, test statistic ( $t$ value) and the significance ( $P$ value). Significant terms $(P<0.05)$ are indicated in bold, and trends $(P=0.05-0.10)$ are indicated in italics.

\begin{tabular}{lcccc}
\hline & Estimate & $\mathbf{d f}$ & $\mathbf{t}$ value & P value \\
\hline Minimal adequate model & & & & \\
Intercept & 1836.85 & 27.30 & - & - \\
Bio female personality & 203.88 & 38.33 & 1.67 & 0.10 \\
Bio male personality & -66.55 & 41.21 & -0.76 & 0.45 \\
Sex & -124.71 & 106.86 & -4.23 & $<0.001$ \\
Catch time & 38.01 & 40.29 & 1.16 & 0.25 \\
Catch date & 280.97 & 30.00 & 3.26 & $\mathbf{0 . 0 0 3}$ \\
Year & -398.11 & 27.81 & -3.43 & 0.002 \\
Brood size & -7.86 & 38.80 & -0.32 & 0.75 \\
Bio female personality * Bio male personality & 445.54 & 35.80 & 2.41 & 0.02 \\
Bio female personality * year & -147.69 & 42.00 & -1.15 & 0.26 \\
Bio male personality * year & 71.57 & 43.11 & 0.75 & 0.46 \\
Year * catch date & -148.15 & 30.68 & -1.37 & 0.18 \\
Bio female personality * Bio male personality * year & -469.94 & 36.35 & -2.49 & $\mathbf{0 . 0 2}$ \\
\hline
\end{tabular}


Table S6: Offspring fledging weight foster parents. Table consists of all variables tested in the linear mixed model with the offspring weight at 14 days old as the dependent variable ( $N$ offspring $=133$, broods $=$ 28). Given is the estimate, degrees of freedom (df), test statistic ( $t$ value) and the significance ( $P$ value). Significant terms $(P<0.05)$ are indicated in bold, and trends $(P=0.05-0.10)$ are indicated in italics.

\begin{tabular}{lcccc}
\hline & Estimate & df & t value & P value \\
\hline Minimal adequate model & & & & \\
Intercept & 1530.10 & 53.52 & - & - \\
Sex & -50.93 & 213.81 & -2.42 & 0.02 \\
Catch time & 16.44 & 50.85 & 0.57 & 0.57 \\
Catch date & 8.65 & 60.19 & 0.17 & 0.87 \\
Year & -58.69 & 51.95 & -1.04 & 0.30 \\
Brood size & 13.43 & 57.62 & 0.52 & 0.61 \\
Egg weight & 8.39 & 51.01 & 0.49 & 0.63 \\
Hatch date * year & 38.02 & 57.31 & 0.61 & 0.55 \\
\hline Dropped terms & & & & \\
Foster female personality * Foster male personality * year & -6.04 & 13.56 & -0.02 & 0.98 \\
Foster male personality * year & 144.63 & 18.94 & 1.06 & 0.30 \\
Foster female personality * year & 206.79 & 19.01 & 1.64 & 0.12 \\
Foster female personality * Foster male personality & -67.06 & 19.24 & -1.19 & 0.25 \\
Foster female personality & 28.68 & 19.94 & 0.57 & 0.58 \\
Foster male personality & 56.51 & 30.87 & 1.70 & 0.10 \\
\hline
\end{tabular}

Table S7: Offspring fledging probability biological parents. Table consists of all variables tested in the generalised linear mixed model with the offspring fledging probability (0/1) as the dependent variable ( $N$ offspring $=136$, broods $=33$ ). Given is the estimate, test statistic $(Z$ value) and the significance ( $P$ value). Significant terms $(P<0.05)$ are indicated in bold, and trends $(P=0.05-0.10)$ are indicated in italics.

\begin{tabular}{lccc}
\hline & Estimate & Z value & P value \\
\hline Minimal adequate model & & & \\
Intercept & 0.95 & - & - \\
Year & -0.78 & -0.86 & 0.39 \\
Hatch date & 1.73 & 1.56 & 0.12 \\
Brood size & -0.44 & -1.28 & 0.20 \\
Year ${ }^{*}$ hatch date & -1.46 & -1.21 & 0.23 \\
\hline Dropped terms & & & \\
Bio female personality * Bio male personality * year & 0.21 & 0.06 & 0.95 \\
Bio male personality * year & -0.05 & -0.03 & 0.97 \\
Bio female personality * Bio male personality & 0.56 & 0.85 & 0.40 \\
Bio male personality & 0.55 & 1.35 & 0.18 \\
Bio female personality * year & -1.36 & -1.23 & 0.22 \\
Bio female personality & 0.14 & 0.28 & 0.78 \\
\hline
\end{tabular}


Table S8: Offspring fledging probability foster parents. Table consists of all variables tested in the generalised linear mixed model with the offspring fledging probability (0/1) as the dependent variable ( $N$ offspring $=133$, broods $=28)$. Given is the estimate, test statistic $(Z$ value) and the significance ( $P$ value). Significant terms $(P<0.05)$ are indicated in bold, and trends $(P=0.05-0.10)$ are indicated in italics.

\begin{tabular}{lccc}
\hline & Estimate & Z value & P value \\
\hline Minimal adequate model & & & \\
Intercept & 0.90 & - & - \\
Sex & -0.20 & -0.71 & 0.48 \\
Hatch date & 0.48 & 1.32 & 0.19 \\
Year & 0.64 & 0.84 & 0.40 \\
Brood size & -0.19 & -0.78 & 0.44 \\
\hline Dropped terms & & & \\
Foster male personality * Foster female personality * year & -5.16 & -1.56 & 0.12 \\
Year * hatch date & -0.27 & -0.24 & 0.81 \\
Foster male personality * year & -0.86 & -0.65 & 0.52 \\
Foster male personality * Foster female personality & -0.54 & -0.86 & 0.39 \\
Foster female personality * year & 1.59 & 1.40 & 0.16 \\
Foster male personality & -0.09 & -0.22 & 0.82 \\
Foster female personality & 0.05 & 0.11 & 0.92 \\
\hline
\end{tabular}


Table S9 and S10: Offspring fledging weight. Tables consist of all variables tested in the two linear mixed models with the offspring weight on at 14 days old as the dependent variable ( $N$ offspring $=133$, broods $=28$ ). Model S9 tests the effects of the total number of visits and average prey volume on offspring fledging weight. Model S10 tests the effects of total prey volume on fledging weight. Given is the estimate, degrees of freedom (df), test statistic ( $t$ value) and the significance (P value). Significant terms $(P<0.05)$ are indicated in bold, and trends $(P=0.05-0.10)$ are indicated in italics.

Table S9: Offspring fledging weight - Total number of visits / average prey volume

\begin{tabular}{lcccc}
\hline & Estimate & df & t value & P value \\
\hline Minimal adequate model & & & & \\
Intercept & 1535.36 & 34.86 & - & - \\
Average prey volume & -46.13 & 32.09 & -1.79 & 0.08 \\
Offspring sex & -67.42 & 170.74 & -2.83 & $\mathbf{0 . 0 1}$ \\
Catch time & 4.44 & 34.85 & 0.15 & 0.88 \\
Catch date & 64.50 & 31.91 & 2.37 & $\mathbf{0 . 0 2}$ \\
Year & -64.80 & 31.76 & -1.04 & 0.31 \\
Brood size & -17.40 & 33.99 & -0.63 & 0.53 \\
\hline Dropped terms & & & & \\
Total number of visits & 32.10 & 32.16 & 1.04 & 0.31 \\
\hline
\end{tabular}

Table S10: Offspring fledging weight - total prey volume

\begin{tabular}{lcccc}
\hline & Estimate & $\mathbf{d f}$ & $\mathbf{t}$ value & P value \\
\hline Minimal adequate model & & & & \\
Intercept & 1530.64 & 59.08 & - & - \\
Offspring sex & -59.94 & 251.44 & -3.03 & $<\mathbf{0 . 0 0 1}$ \\
Catch time & 18.89 & 57.47 & 0.76 & 0.45 \\
Catch date & 40.16 & 56.05 & 1.57 & 0.12 \\
Year & -54.89 & 56.38 & -1.06 & 0.29 \\
Brood size & 14.52 & 65.05 & 0.64 & 0.52 \\
\hline Dropped terms & & & & \\
Total prey volume & 2.14 & 33.25 & 0.07 & 0.94 \\
\hline
\end{tabular}


Tables S11 and S12: Offspring fledging probability. Table consists of all variables tested in the generalised linear mixed model with the offspring fledging probability (0/1) as the dependent variable ( $N$ offspring $=133$, broods $=28$ ). Model $S 9$ tests the effects of the total number of visits and average prey volume on fledging probability. Model S10 tests the effects of total prey volume on fledging probability. Given is the estimate, test statistic ( $Z$ value) and the significance $(P$ value). Significant terms $(P<0.05)$ are indicated in bold, and trends $(P=0.05-0.10)$ are indicated in italics.

Table S11: Offspring fledging probability - Total number of visits / average prey volume

\begin{tabular}{lccc}
\hline & Estimate & Z value & P value \\
\hline Minimal adequate model & & & \\
Intercept & -0.49 & - & - \\
Offspring sex & -0.20 & -0.71 & 0.48 \\
Hatch date & 0.48 & 1.32 & 0.19 \\
Year & 0.64 & 0.84 & 0.40 \\
Brood size & -0.26 & -0.78 & 0.44 \\
\hline Dropped terms & & & \\
Total prey volume & 0.16 & 0.65 & 0.52 \\
\hline
\end{tabular}

Table S12: Offspring fledging probability - Total prey volume

\begin{tabular}{lccc}
\hline & Estimate & Z value & P value \\
\hline Minimal adequate model & & & \\
Intercept & 4.82 & - & - \\
Total number of visits & 0.55 & 2.26 & $\mathbf{0 . 0 2}$ \\
Offspring sex & 0.07 & 0.21 & 0.83 \\
Hatch date & 0.89 & 2.86 & $<\mathbf{0 . 0 0 1}$ \\
Year & -0.23 & -0.40 & 0.69 \\
Brood size & -0.55 & -2.52 & $\mathbf{0 . 0 1}$ \\
\hline Dropped terms & & & \\
Average prey volume & -0.10 & -0.40 & 0.69 \\
\hline
\end{tabular}




\section{Appendix B - Model tables using AICc method (model averaged)}

Since analyses using backwards elimination may be susceptible to the order in which variables are eliminated from the models, we compared the results from the backwards elimination models with the results using an information criterion model selection approach.

\section{Provisioning}

For all models on provisioning behaviour (average prey volume, visit rate, total prey volume and working day length; tables S9-S12), following a model selection approach, we calculated for each iteration and each model Akaike's information criterion corrected for small sample sizes (AICc; Burnham and Anderson 2002), giving an average AICc (averaged over 1000 iterations) for each model. These average AICc values for each model were rescaled $(\triangle \mathrm{AICc})$ calculating the difference between the AICc value of each model and the minimum AICc obtained among all competing models (i.e. the best model has $\triangle \mathrm{AICC}=0$ ). Models with $\triangle \mathrm{AICc} \leq 2$ were considered equivalent (Burnham and Anderson 2002). In cases where model selection as a function of AICc did not give a single model, we performed an averaging of equivalent models (i.e. models with $\Delta \mathrm{AICc} \leq 2$; Burnham and Anderson 2002). We calculated the mean of the predictor estimates, their unconditional standard errors and confidence intervals $(\mathrm{CI})$, and the relative importance of each variable in the final averaged model ( $\Sigma \omega$ i, the sum of Akaike weights of models with $\triangle \mathrm{AICc} \leq 2$ in which the variable was included). Parameter estimates were considered significant when their 95\% CI did not span zero (Burnham and Anderson 2002). Model selection was performed using the $R$ packages lme4, AICcmodavg and MuMIn (Bates et al. 2015; R Development Core Team 2015; Mazerolle 2017). 
Table S9: Average prey volume (model selection approach).

\begin{tabular}{lccccc}
\hline & Full & Subset & $\boldsymbol{\Sigma}$ weights & SE & 95\% CI for subset $\boldsymbol{\beta}$ \\
\hline Intercept & 281.34 & 281.34 & 1.00 & 0.61 & {$[241.24,317.21]$} \\
Brood size & -1.40 & -1.40 & 1.00 & 0.40 & {$[-25.87,22.25]$} \\
Date & -46.49 & -46.49 & 1.00 & 0.35 & {$[-68.16,-24.25]$} \\
Date * year & 38.74 & 38.74 & 1.00 & 0.51 & {$[\mathbf{7 . 6 2 , 7 0 . 4 1 ]}$} \\
Mate personality & -2.64 & -30.63 & 0.09 & 0.48 & {$[-65.11,-4.81]$} \\
Personality & -37.26 & -76.06 & 0.49 & 1.00 & {$[-144.9,-35.42]$} \\
Personality ${ }^{*}$ year & 22.56 & 95.00 & 0.24 & 1.55 & {$[\mathbf{1 1 . 2 , 2 0 0 . 8 8 ]}$} \\
Sex & 5.54 & 49.03 & 0.11 & 1.58 & {$[-43.56,135.22]$} \\
Visit rate & -20.99 & -48.23 & 0.44 & 0.52 & {$[-84.65,-18.07]$} \\
Year & -47.33 & -47.33 & 1.00 & 0.75 & {$[-91.71,0.75]$} \\
\hline
\end{tabular}

Table S10: Visit rate (model selection approach)

\begin{tabular}{lccccc}
\hline & Full & Subset & $\boldsymbol{\Sigma}$ weights & SE & $\mathbf{9 5 \%}$ CI for subset $\beta$ \\
\hline Intercept & 18.87 & 18.87 & 1.00 & 0.07 & {$[15.94,21.75]$} \\
Brood size & 2.37 & 2.37 & 1.00 & 0.06 & {$[-0.28,4.77]$} \\
Date & -0.74 & -0.74 & 1.00 & 0.04 & {$[-2.65,0.98]$} \\
Date * year & 3.83 & 3.83 & 1.00 & 0.09 & {$[0.22,7.52]$} \\
Mate personality & -0.58 & -2.20 & 0.26 & 0.07 & {$[-5.37,0.59]$} \\
Personality & -1.22 & -3.22 & 0.38 & 0.06 & {$[-6.25,-0.71]$} \\
Prey volume & -2.28 & -3.82 & 0.60 & 0.06 & {$[-6.54,-1.7]$} \\
Sex & 0.24 & 1.78 & 0.13 & 0.17 & {$[-5.47,9.06]$} \\
Year & 4.72 & 4.72 & 1.00 & 0.11 & {$[0.25,9.46]$} \\
\hline
\end{tabular}


Table S11: Total prey volume (model selection approach)

\begin{tabular}{lccccc}
\hline & Full & Subset & $\boldsymbol{\Sigma}$ weights & SE & 95\% CI for subset $\beta$ \\
\hline Intercept & 10325.94 & 10325.94 & 1.00 & 24.85 & {$[8906.47,11618.85]$} \\
Brood size & 626.63 & 626.63 & 1.00 & 18.64 & {$[-540.21,1530.24]$} \\
Date & -1457.76 & -1457.76 & 1.00 & 16.36 & {$[-2528.94,-712.51]$} \\
Date * year & 2338.49 & 2338.49 & 1.00 & 23.65 & {$[958.97,3547.79]$} \\
Mate personality & -551.67 & -1491.24 & 0.37 & 31.66 & {$[-3525.98,110.14]$} \\
Personality & -1133.45 & -2057.17 & 0.55 & 23.87 & {$[-3672.43,-990.54]$} \\
Year & 777.29 & 777.29 & 1.00 & 35.61 & {$[-1442.51,2565.04]$} \\
\hline
\end{tabular}

Table S12: Working day length (model selection approach)

\begin{tabular}{lccccc}
\hline & Full & Subset & $\boldsymbol{\Sigma}$ weights & SE & 95\% CI for subset $\boldsymbol{\beta}$ \\
\hline Intercept & 16.29 & 16.29 & 1.00 & 0.02 & {$[15.16,17.43]$} \\
Brood size & 0.05 & 0.05 & 1.00 & 0.00 & {$[-0.05,0.17]$} \\
Date & -1.47 & -1.47 & 1.00 & 0.03 & {$[-3.36,0.5]$} \\
Date * year & 0.55 & 0.55 & 1.00 & 0.01 & {$[-0.26,1.4]$} \\
Real day length & 1.28 & 1.28 & 1.00 & 0.03 & {$[-0.26,2.93]$} \\
Sex & -0.25 & -0.25 & 1.00 & 0.00 & {$[-0.5,-0.03]$} \\
Year & -1.25 & -1.25 & 1.00 & 0.03 & {$[-2.83,0.39]$} \\
\hline
\end{tabular}




\section{Reproductive success}

Similar to the models on provisioning, the models on reproductive success may also be susceptible to the order in which variables are eliminated when using backwards elimination procedures. Therefore, we also compared these model outcomes to additional models using an information criterion model selection approach (Tables S13-S19). Using this approach we calculated AICc values for models with all possible combinations of variables and determined the model with the lowest AICc. Models with $\triangle \mathrm{AICc} \leq 2$ were considered equivalent (Burnham and Anderson 2002). In cases where model selection as a function of AICc did not give a single model, we performed an averaging of equivalent models (i.e. models with $\triangle \mathrm{AICc} \leq 2$; Burnham and Anderson 2002). Given is the mean of the predictor estimates, their unconditional standard errors and confidence intervals (CI). Parameter estimates were considered significant when their 95\% CI did not span zero (Burnham and Anderson 2002). Model selection was performed using the $R$ packages $l m e 4$, AICcmodavg and MuMIn (Bates et al. 2015; R Development Core Team 2015; Mazerolle 2017).

Table S13: Lay date (model selection approach)

\begin{tabular}{lccc}
\hline & $\beta$ & SE & $\mathbf{9 5 \% ~ C I ~ f o r ~ s u b s e t ~} \beta$ \\
\hline Intercept & 1.76 & 0.87 & {$[0.06,3.46]$} \\
Year & -0.12 & 0.27 & {$[-0.64,0.4]$} \\
Clutch size & -0.22 & 0.10 & {$[-0.42,-0.02]$} \\
Female personality & 0.06 & 0.17 & {$[-0.27,0.39]$} \\
Male personality & 0.05 & 0.14 & {$[-0.23,0.32]$} \\
\hline
\end{tabular}

Table S14: Clutch size (model selection approach)

\begin{tabular}{lccc}
\hline & $\boldsymbol{\beta}$ & $\mathrm{SE}$ & $\mathbf{9 5 \%}$ CI for subset $\boldsymbol{\beta}$ \\
\hline Intercept & 8.37 & 0.31 & {$[7.76,8.97]$} \\
Year & -0.71 & 0.40 & {$[-1.49,0.07]$} \\
Lay date & -0.24 & 0.31 & {$[-0.85,0.37]$} \\
Lay date ${ }^{*}$ year & -0.73 & 0.50 & {$[-1.71,0.24]$} \\
Female personality & 0.38 & 0.49 & {$[-0.57,1.34]$} \\
Female personality ${ }^{*}$ year & -0.97 & 0.53 & {$[-2.01,0.08]$} \\
Male personality & -0.05 & 0.22 & {$[-0.48,0.37]$} \\
\hline
\end{tabular}


Table S15: Average egg weight (model selection approach)

\begin{tabular}{lccc}
\hline & $\boldsymbol{\beta}$ & SE & $\mathbf{9 5 \%}$ CI for subset $\boldsymbol{\beta}$ \\
\hline Intercept & 1.35 & 0.19 & {$[0.97,1.73]$} \\
Year & 0.00 & 0.06 & {$[-0.12,0.11]$} \\
Clutch size & 0.03 & 0.02 & {$[-0.01,0.08]$} \\
Lay date & 0.06 & 0.04 & {$[-0.02,0.14]$} \\
Lay date ${ }^{*}$ year & -0.13 & 0.07 & {$[-0.26,0.01]$} \\
Male personality & 0.03 & 0.03 & {$[-0.03,0.09]$} \\
Female personality & -0.02 & 0.04 & {$[-0.09,0.05]$} \\
\hline
\end{tabular}

Table S16: Effect of biological parents on offspring weight (model selection approach)

\begin{tabular}{lccc}
\hline & $\boldsymbol{\beta}$ & SE & $\mathbf{9 5 \% ~ C I ~ f o r ~ s u b s e t ~} \beta$ \\
\hline Intercept & 1556.81 & 61.16 & {$[1436.93,1676.69]$} \\
Sex & -124.71 & 29.81 & {$[-\mathbf{1 8 3 . 1 2}, \mathbf{- 6 6 . 2 9 ]}$} \\
Catch time & 38.01 & 33.20 & {$[-27.06,103.09]$} \\
Catch date & 225.12 & 69.84 & {$[\mathbf{8 8 . 2 4}, 362]$} \\
Year & -47.95 & 70.68 & {$[-186.48,90.59]$} \\
Brood size & -7.86 & 25.14 & {$[-57.14,41.41]$} \\
Female personality & 202.09 & 122.80 & {$[-38.58,442.77]$} \\
Male personality & -67.06 & 88.14 & {$[-239.81,105.69]$} \\
Catch date ${ }^{*}$ year & -142.85 & 80.99 & {$[-301.58,15.88]$} \\
Female personality ${ }^{*}$ male personality & 445.37 & 186.45 & {$[79.94,810.8]$} \\
Female personality ${ }^{*}$ year & -145.86 & 129.09 & {$[-398.86,107.14]$} \\
Male personality ${ }^{*}$ year & 72.11 & 96.99 & {$[-117.98,262.21]$} \\
Female personality ${ }^{*}$ male personality ${ }^{*}$ year & -469.77 & 190.46 & {$[-843.07,-96.47]$} \\
\hline
\end{tabular}


Table S17: Effect of foster parents on offspring weight (model selection approach)

\begin{tabular}{lccc}
\hline & $\boldsymbol{\beta}$ & SE & $\mathbf{9 5 \% ~ C I ~ f o r ~ s u b s e t ~} \boldsymbol{\beta}$ \\
\hline Intercept & 1516.19 & 73.59 & {$[1371.95,1660.42]$} \\
Sex & -41.83 & 31.03 & {$[-102.63,18.98]$} \\
Catch time & 17.31 & 49.33 & {$[-79.36,113.99]$} \\
Catch date & 101.04 & 89.00 & {$[-73.4,275.48]$} \\
Year & -43.48 & 90.47 & {$[-220.8,133.84]$} \\
Brood size & 38.25 & 40.31 & {$[-40.77,117.25]$} \\
Foster male personality & -49.30 & 117.91 & {$[-280.4,181.8]$} \\
Catch date * year & -34.61 & 102.94 & {$[-236.37,167.14]$} \\
Foster male personality * year & 101.53 & 123.59 & {$[-140.7,343.75]$} \\
Foster female personality & 31.84 & 44.61 & {$[-55.6,119.28]$} \\
\hline
\end{tabular}

Table S18: Effect of biological parents on fledging probability (model selection approach)

\begin{tabular}{lccc}
\hline & $\boldsymbol{\beta}$ & SE & $\mathbf{9 5 \%}$ CI for subset $\boldsymbol{\beta}$ \\
\hline Intercept & 1.23 & 0.79 & {$[-0.32,2.78]$} \\
Sex & -0.47 & 0.44 & {$[-1.33,0.39]$} \\
Hatch date & 1.82 & 1.14 & {$[-0.41,4.05]$} \\
Year & -0.80 & 0.94 & {$[-2.64,1.04]$} \\
Brood size & -0.44 & 0.35 & {$[-1.12,0.25]$} \\
Hatch date *year & -1.60 & 1.24 & {$[-4.02,0.82]$} \\
Male personality & 0.43 & 0.44 & {$[-0.44,1.3]$} \\
Female personality & 0.15 & 0.52 & {$[-0.86,1.17]$} \\
\hline
\end{tabular}


Table S19: Effect of foster parents on fledging probability (model selection approach)

\begin{tabular}{|c|c|c|c|}
\hline & $\beta$ & SE & $95 \% \mathrm{CI}$ for subset $\beta$ \\
\hline Intercept & -1.97 & 1.34 & {$[-4.59,0.65]$} \\
\hline Sex & -0.05 & 0.48 & {$[-1,0.89]$} \\
\hline Hatch date & 3.12 & 1.77 & {$[-0.35,6.59]$} \\
\hline Year & 2.62 & 1.44 & {$[-0.2,5.43]$} \\
\hline Brood size & -0.77 & 0.49 & {$[-1.73,0.19]$} \\
\hline Foster female personality & -2.86 & 1.41 & {$[-5.61,-0.1]$} \\
\hline Hatch date ${ }^{*}$ year & -3.25 & 1.86 & {$[-6.89,0.4]$} \\
\hline Foster female personality ${ }^{*}$ year & 2.89 & 1.52 & {$[-0.08,5.86]$} \\
\hline Foster male personality & -0.19 & 0.41 & {$[-0.99,0.61]$} \\
\hline
\end{tabular}



Chapter 6

General discussion 
7 he amount of sexual-selection literature is enormous, covering a wide range of 1 specialised topics: different species, mating systems, theories and approaches. While most theories and studies assume population-wide preferences, more and more studies suggest that individuals may also differ in their preference. Instead of preferring a universally 'best' individual, they may prefer a mate that is best for them: a compatible mate. Moreover, it has been shown that individuals also gain benefits from compatibility with their mate. Therefore, in this thesis I investigated individual differences and the role of compatibility in mate preferences in great tits. To gain a better insight into the role of compatibility in sexual selection I addressed the following three questions in this thesis:

- Do individuals differ in their preferences for a mate?

- How are mate preferences reflected in mate choice?

- How does pair (in)compatibility affect reproductive success?

Here, I address these questions in light of the results presented here as well as the findings of other studies. Additionally, since the methods used in measuring mate preferences and mate choice can have a major impact on the conclusions that we draw on the process of selection, I will discuss how my methods of measuring preference and choice, as well as how individuals evaluate potential partners, may affect the results and conclusions. Subsequently I will draw conclusions on what the results might mean for the study of sexual selection in a broader sense and make some suggestions for promising directions for future research.

\section{Measuring mate preferences and mate choice}

When studying mate preferences, mate choice and sexual selection it is important to consider how our experimental design, our observations and our analyses affect the conclusions that we can draw. One very important aspect of this is the distinction between preference and choice. As explained in chapter 1, the actual choice for a mate is the result of mate preferences while under physical and environmental constraints. Therefore, the actual choice may not represent preferences at all. For instance, while in water striders (Heteroptera; Gerridae) assortative mating patterns for size suggest individual differences in mating preferences (Arnqvist et al. 1996), it is the competitive advantage that large males have in intrasexual competition over the largest females that actually causes such mating patterns. All males thus have the same preferences for more fecund large females. On the other hand, in blue tits assortative mating patterns for heterozygosity levels were found (García-navas et al. 2009). The authors suggested that these patterns may be the result of competition over mates with high heterozygosity levels. However, since this study did not measure mate preferences it cannot be concluded what caused these mating 
patterns. The mating patterns I measured here suggest the possibility that these assortative mating patterns previously found in blue tits (García-navas et al. 2009) could be the result of assortative preferences instead (chapter 3). Thus, making assumptions about preferences on the basis of mating patterns alone may yield confounded results. Moreover, to increase our understanding of the selective pressures leading to the evolution of preferences we have to consider preference, choice and the fitness benefits related to these preferences. Therefore I strongly advise that the terminology for mate preference and mate choice should be used consistently to be able to compare studies and draw broad conclusions (Jennions and Petrie 1997; Edward 2015). To our knowledge this study is the first to consider all these three aspects of sexual selection and is able to show how despite constraints on choice mate preferences may still give reproductive benefits (chapter 3 ).

To test preference functions, different designs may be more or less suitable depending on the questions that we want to answer and the species being studied (Wagner 1998; Castellano et al. 2012; Dougherty and Shuker 2014). The most common designs used to test preferences are the no-choice or sequential test, and the (simultaneous) choice-test. Whereas Wagner (1998) suggests that only sequential choice designs are able to measure real preferences without the interference of motivation to choose, the disadvantage of this design is that individuals may perceive this as a 'last-chance option'. Without any knowledge of the availability of mates, and the possibility to compare, their responses may be the same for every stimulus presented since they may perceive it as their only option. Additionally, these sequential tests may be very sensitive to order effects. On the other hand, in simultaneous choice tests, it may be more difficult to discern non-linear preferences and the social context may influence responses both in motivation and in the preference function measured. In chapter 2 I found that indeed this social context affected the responses of choosers. The average attractiveness of the stimulus groups affected the total time individuals spent visiting all stimulus birds, and the variation in attractiveness within groups affected the measured preference slopes. This indicates that great tits use a combination of both absolute and comparative evaluation and that the social context indeed affects the measured responses to stimulus groups. Since great tits pair up over the winter, during which time they often forage in large foraging flocks, they are very likely to encounter potential mates in a context where comparison is possible (Culina 2014). Being able to value potential partners comparatively as a cognitive shortcut when possible, while still having an absolute notion of the attractiveness of an individual when it is encountered alone may benefit the behavioural flexibility of these birds in order to make an informed choice in different settings. Similar results of individuals being able to use both absolute and comparative evaluation have been found in other species by testing individuals for their preferences using both sequential and simultaneous choice designs 
(Brandt et al. 2005; Maclaren and Rowland 2016). For instance, in female sailfin mollies the direction of their preferences was the same over both tests, but, depending on the experimental paradigm, the measured preferences were found to be stronger or weaker (Maclaren and Rowland 2016). Therefore, when designing mate preference tests, the expected evaluation method of the study species and the effects that the different choice designs may have on the measurement of the preferences should be kept in mind. Additionally, depending on how the study species evaluates the stimuli, taking the social context in account in the analyses may yield more reliable measures of preferences.

\section{Do individuals differ in their preferences for a mate?}

This study can be added to the increasing number of studies that provide evidence that individuals can differ in their preferences for a mate. The results presented in this thesis suggest that individual great tits indeed differ in their preferences for a mate. In chapter 4 I have found that there were no population-wide preferences for any of the stimulus traits measured. Additionally, I have found that individual preference slopes for (at least three of) these traits differed between individuals. Moreover, in chapter 3 I have shown that preferences for certain traits may be dependent on the choosers' own traits, suggesting preference for the compatibility of these traits.

The absence of uniform preferences for any of the measured traits is quite striking. Despite the fact that in this species with biparental care I expected compatibility to be of importance, some traits might have been indicators of qualities that would be beneficial to all choosers. However, here, for the traits that I measured, I found no population-wide preferences. Therefore there may not be a 'best individual' or a 'universal Prince Charming', and potential mates cannot simply be ordered into high or low quality mates (Rosenthal 2017). Instead, these results show that the optimal mate is individual-specific and individuals prefer the mate that is best for them.

Here, I measured differences in preferences on two different levels. In chapter 3 I measured preferences at a population level, depending on the choosers' traits. Subsequently, I studied whether these preferences were reflected in the mating patterns or in reproductive success. In a way, this is very similar to studies on population-wide preferences: we measure a preference pattern and assume that individuals that find a mate according to that pattern find their mate attractive. For this population-wide approach, we compare population-wide preference patterns with patterns in mate choice and breeding success. Therefore, it is not necessary to have both the preference function and the breeding data for each individual considered in the analysis. We can simply use all individuals measured and compare patterns. Although this approach enables us to use 
more individuals, since we're measuring population-wide patterns and not individual based measurements, it also generalises some of our assumptions. By studying such population-wide preferences for compatibility, it is possible to study the benefits that individuals can gain from mating according to these preferences through the effects of pair compatibility for these traits, and thereby increase our understanding of how and why these preferences may have evolved. In this case these patterns show that in this population preferences for heterozygosity depend on the choosers' traits in a similar way. Additionally, a trend for preference for moderate levels of relatedness suggests that choosing individuals might be able to assess their relatedness with regard to other individuals as well and that this plays a role in their preferences. Such self-referent preferences suggest that individuals search for a mate that is compatible (DechaumeMoncharmont et al. 2013; Weddle et al. 2013).

In chapter 4 , in contrast to the population-wide preferences in chapter 3, I estimated preference functions for each individual separately. Here I found that individuals differed in their preference slopes for (at least) three of the measured traits (heterozygosity, yellowness and breast stripe size). On the basis of these individual preference functions I was able to estimate the individual mate attractiveness score (IMA). This is a measure of how attractive each particular focal individual perceives its mate (or other individuals of the opposite sex), according to each of the traits I measured. The approach used in chapter 3 can increase our understanding of the benefits of preferences for these traits and may shed light on why they might have evolved. However, the individual-based approach in chapter 4 can give us a better understanding of the effects of perceived attractiveness on reproductive success. Where quite a number studies in captivity on a wide range of study systems have shown that perceived mate attractiveness affects reproductive success by experimentally pairing individuals with either attractive or unattractive mates (Koeninger Ryan and Altmann 2001; Drickamer et al. 2003; Gowaty et al. 2003; Anderson et al. 2007; Ihle et al. 2015), such effects have so far not been found in wild populations. In the studies performed in captivity, individuals are typically paired with either preferred or unpreferred mates, which subsequently affects their reproductive success. Similarly, in the wild, due to constraints on choice, individuals may also end up pairing with partners that don't meet their preferences entirely. Therefore, estimating how attractive individuals perceive their mate to be could give very interesting insights into the consequences of individual mate attractiveness, regardless of the actual trait values.

In many modelling studies, preferences for one trait - or multiple traits signalling the same quality - are often assumed. In the case of multiple traits, assessing each of these traits separately would increase the sampling costs, making the evolution of preferences for multiple traits signalling the same quality unlikely (Kuijper et al. 2012). However, it is very 
well possible that choosers use different traits as signals for different aspects of 'quality' (Johnstone 1995; Candolin 2003; van Doorn and Weissing 2004). Theoretical models indicate that when different ornaments indicate different qualities, preferences for multiple traits may indeed evolve, even when this increases the sampling costs (van Doorn and Weissing 2004). Our results in chapter 3 show that also for genetic traits individuals may use multiple traits, relatedness and heterozygosity, for their preferences. Additionally, in chapter 4 I estimated preference functions for each individual and for each trait that affected the time they spent with stimulus birds. For most individuals their association time was determined by more than one trait. This fact that choosers can select for multiple traits, and that these different traits signal different messages has been found in a number of studies. For instance, in the three-spined stickleback (Gasterosteus aculeatus), females can use both visual and olfactory cues as signals in choosing a mate. Where olfactory cues are used to optimise offspring MHC (major histocompatibility complex) allele numbers (Reusch et al. 2001; Aeschlimann et al. 2003), visual cues can be used to indicate direct benefits such as parental care (Bakker and Milinski 1993; Kraak 1999) or indirect benefits in the form of good or attractive genes (Milinski and Bakker 1990; Bakker 1993). Here I found that females may have preferences for both heterozygosity and intermediate levels of relatedness (chapter 3 ). These two traits appear to indicate different benefits. These results suggest a direct benefit for heterozygosity compatibility, while on the other hand choice for intermediate levels of relatedness may increase offspring heterozygosity while avoiding any detrimental effects of too extreme outbreeding (Bateson 1982; Neff 2004; Greeff et al. 2009; Richard et al. 2009). Although I found no benefits of offspring heterozygosity on reproductive success, which is likely to depend on optimal levels of parental relatedness, it is possible that these indirect benefits only appear later in life (Szulkin and Sheldon 2007). Moreover, choosers weighed the heterozygosity of that individual differently depending on their relatedness with a certain stimulus individual, suggesting that different traits may indicate different benefits, which, depending on the choosers traits may be more or less important (Candolin 2003; Burley et al. 2018).

Furthermore, in chapter 3 I found similar mate preferences for both males and females: males and female preference patterns did not differ. Especially since both sexes invest heavily in reproduction, both may benefit from finding a compatible mate (Kokko and Johnstone 2002). The possibility of mutual choice however has not been studied very extensively so far. In zebra finches it has been found that males prefer fecund females (Jones et al. 2001), and that males may invest differently in mating depending on female fecundity (Engqvist and Sauer 2001). Males may also try to obtain a compatible mate for instance through mutual mate choice or differential courtship efforts (e.g. through 
specifically courting certain females or investing more in courtship behaviours for certain females). Great tits only show limited dimorphism and in such a case female ornamentation is often suggested to be merely a by-product of selection for male traits (Amundsen and Forsgren 2001). However, in species where mutual mate choice may be of importance, female traits may signal similar qualities as male signals enabling also male selection of females on the basis of these traits (Amundsen 2000). And this may also be a reason why here I found no differences between male and female preference patterns.

\section{How are mate preferences reflected in mate choice?}

Individuals look for a mate on the basis of their preferences but have to choose a mate under physical and environmental constraints (see introduction Figure 1.1). In this thesis I was only able to study the result of the process of mate choice. In chapters 3 and 4 I compared preferences with mating patterns. In chapter 3 I show that assortative preferences for heterozygosity were not reflected in the mating patterns and in chapter 4 I show that the individual mate attractiveness scores within pairs are not higher than when they would have mated at random. This suggests that, despite having preferences for certain traits in a mate, great tits did not obtain a mate with traits that met these preferences. However, it is possible that individuals still chose according to their preferences from the options that they sampled, which is dependent on the availability and distribution of potential mates. In great tits individuals become available (e.g. after divorce or the death of a partner) and unavailable (after mating) throughout the winter and thus the availability of mates may also vary across time and locations (Hinde 1952; Gosler 1993; Culina 2014). Unfortunately, on the basis of our data, I cannot conclude anything about which potential mates were available and which potential mates were sampled before choosing a pair mate. Additionally it is possible that under social monogamy selection may not favour high levels of choosiness because the benefits of unidirectional choosiness may not exceed the costs related to scramble competition (Dechaume-Moncharmont et al. 2016; Wang et al. 2017). Under such circumstances individuals are expected to have very low acceptance thresholds or to sample two individuals at most in the population. These low levels of choosiness and sampling effort may lead to very subtle mating patterns and may explain the absence of mating patterns in our observations. However, despite the fact that I did not find any indication of mating according to their preferences, our data is not sufficient to draw definite conclusions about an absence or presences of any mating patterns. As long as having preferences slightly increases the chances of finding a compatible mate, and the benefits of compatibility outweigh the costs of mate selection, selection would still favour the evolution of preferences (Rosenthal 2017). 


\section{How does pair (in)compatibility affect reproductive success?}

\section{Trait compatibility}

The reproductive benefits that individuals may gain from mate choice can be direct or indirect. In this study I found both types of benefits of choice for certain traits in a mate.

\section{Genetic compatibility}

In chapter 3 I found that, despite my hypothesis that especially homozygous individuals would obtain genetic benefits from finding an unrelated partner in order to increase their offspring heterozygosity, in reality, when biological mothers were homozygous, chicks had a higher fledging probability when she mated with a related male. These compatibility effects of the biological parents were surprising, especially since I expected the genetic characteristics to have a positive effect on offspring performance through offspring heterozygosity. However, I also found no benefit of offspring heterozygosity on chick weight or fledging probability. Thus, possibly these indirect benefits of pair compatibility and the resulting potential fitness effects of heterozygosity or attractiveness in the offspring will only become apparent later in life (Szulkin et al. 2007). To get a better insight into the evolution of sexual selection for this genetic compatibility it may therefore be needed to follow offspring throughout their lives instead of only during the nestling period like I did here. Thus, the genetic benefits that I found in chapter 3 for homozygous females paired with related males, or heterozygous females paired with unrelated males, may be the result of differential allocation by the female in egg size (Horváthová et al. 2012), yolk carotenoids (Marri and Richner 2014) or yolk androgens (Kingma et al. 2009; see also below for differential allocation).

Since choice is costly, the slightest cost can override indirect benefits of choosiness leading, theoretically, to the expectation that sexual selection driven by only indirect benefits may be rare in nature (Kirkpatrick and Barton 1997; Cameron et al. 2003). Perhaps more likely to evolve is direct selection: the evolution of chooser preferences is easier to explain if choosers gain direct benefits from being choosy (Kuijper et al. 2012). A log likelihood ratio test on the random effects in models on offspring weight and offspring fledging probability (chapter 3 ) also indicates that the effects of foster parents on fledging probability and the weight of offspring is much larger than the effect of biological parents (results not presented here). This is consistent with other studies on blue tits (Hadfield et al. 2006) and the common yellowthroat (Geothlypis trichas; Whittingham and Dunn 2005), which show that social father identity is more important for offspring weight than the genetic father identity. It is of course possible that these beneficial effects of genetic parent identity only become apparent later in life, through a higher adult survival or increased reproductive success, which I did not measure in this study. Nevertheless these direct 
effects on offspring fledging probability and offspring weight, and with it chances of surviving the first winter (Van Balen 1973), will therefore have a major effect on the reproductive success and fitness of these pairs. Thus in species with biparental care the direct benefits may simply weigh more heavily than indirect benefits (Alonzo 2012). This is also reflected in our results on reproductive success where I found direct effects of genetic compatibility for heterozygosity in the foster parents, and in the relatedness of the foster parents. Rather than the predominantly indirect effects I expected from genetic compatibility, I also found it to affect rearing success. Although we found preferences for, and benefits of pairing assortatively for heterozygosity, it is unclear why exactly these pairs are more successful at rearing their offspring. Whether these pairs for instance were behaviourally more compatible or invested more when they were able to obtain a mate they preferred, regardless of its apparent heterozygosity or quality (Drickamer et al. 2003; Ihle et al. 2015) can unfortunately not be determined from our data.

Nevertheless, the two effects, direct and indirect benefits of compatibility, need not be mutually exclusive. More complicated patterns of chooser preferences may occur when courters not only differ in parental ability, but also in genetic quality, and these aspects may or may not be correlated. Therefore it matters which aspects of quality are signalled and how to interpret these different signals (Kokko 1998; Alonzo 2012).

\section{Behavioural compatibility}

Another aspect for which I expected compatibility to play a large role, especially in terms of direct benefits is personality. The compatibility of behavioural traits has been shown to be important for mate choice, and to increase reproductive success (Spoon et al. 2006; Schuett et al. 2011; Gabriel and Black 2012; Ouyang et al. 2014). Therefore, in chapter 5 I studied whether this increased reproductive success for behaviourally compatible pairs could be caused by more optimal provisioning behaviour. Here I found that the combination of personalities in a pair did not affect their provisioning behaviour. Instead, I found that although all birds faced a trade-off between nest visit rate and prey volume, fast birds brought in smaller prey for a given visit rate resulting in a lower overall amount of prey delivered to their offspring. These differences in provisioning strategy led to faster explorers bringing a lower total prey volume to their offspring. Thus, personality may certainly play a role in the direct benefits that individuals gain from finding a mate, however, this benefit was not dependent on the choosers' own traits. In contrast to other studies (Budaev et al. 1999; Sinn et al. 2006; Schuett et al. 2011; Ariyomo and Watt 2013; Class et al. 2014, but see Both et al. 2015), I did not find that assortative pairs were better parents; similar pairs were not more successful in rearing their offspring. Similarly, I did not find any other personality related differences in reproductive success. This may be 
caused by the fact that in great tits the effects of personality and behavioural compatibility on fitness and reproductive success have been suggested to fluctuate between years (Dingemanse et al. 2004; Both et al. 2005; Nicolaus et al. 2016). Thus, differences in the occurrence and stability of assortative mating patterns between populations may be correlated with the extent to which the benefits of assortative mating are either stable or context dependent. For instance some studies show that the benefits of assortative mating for personality over disassortative mating are related to how pairs cope with challenging situations (David et al. 2011; Harris and Siefferman 2014). Since I only measured base-line provisioning on one day, on the basis of our data it was not possible to distinguish such effects. This context dependence of these compatibility effects may also in part be an explanation for why I did not find assortative mating patterns (although as said before, this may also be the result of other factors such as territory quality (Drent 1983), the availability of potential mates or female-female interactions). More long-term studies or experimental studies manipulating the environmental context might be able to give better insight into how selection pressures may favour certain combinations of personalities in a pair under some circumstances and other combinations under other circumstances.

Adding to this line of thought about the context dependence of personality effects are the indirect, pre-hatching effects on offspring weight. In one of the two years, original parents that mated assortatively for personality, had a reproductive benefit in terms of offspring fledging weight. This suggests that compatibility for these behavioural traits may have a pre-hatching benefit for offspring in some years, but not in others, and thus that these prehatching effects may also be context dependence. Potentially, offspring with extreme personalities, coming from assortative parents, had a competitive advantage over more intermediate foster siblings, perhaps through different, but equally successful strategies, giving them a higher fledging weight. Or, alternatively this effect on offspring weight works through maternal hormone investment in the eggs (for more on differential allocation see below). The level of androgens deposited has been found to correlate with female personality and her mates' personality (Ruuskanen et al. 2017) and is known to affect offspring early growth and survival. Additionally, since the effects of high androgen levels can have both positive and deleterious consequences on offspring, the resulting net benefits for the offspring are expected to depend on the rearing conditions (Von Engelhardt and Groothuis 2011), which may also explain the context dependence of these pre-hatching effects of parental personality.

\section{Compatibility for attractiveness}

From other studies it is known that individuals have increased reproductive success when they are able to mate with individuals that they perceive as attractive (Koeninger Ryan 
and Altmann 2001; Drickamer et al. 2003; Gowaty et al. 2003; Anderson et al. 2007; Ihle et al. 2015). In chapter 4 however I found no direct or indirect benefits of pairing with attractive mates (a high IMA score) as perceived mate attractiveness was not correlated with pre-hatching investment, fledging weight or fledging probability. Again, this may suggest that individuals did not show preferences for mates with whom they would optimise direct or indirect benefits. However, in chapter 3 I found that preferences for heterozygosity were reflected in the direct benefits of mating according to these preferences. One of the reasons for an absence of a similar effect may be the limited sample size we have for these preference-tested birds, which was also confirmed by a re-analysis of these heterozygosity effects on fledging using this smaller dataset with preferencetested birds. Another reason may lie in the noise in the measurements of the preference functions, which may vary over environments and internal states. However, despite the fact that here, with a limited sample size there were no effects of IMA on reproductive success, this is a very promising method to be able to study the effects of IMA in wild populations. Additionally, it would be very interesting to repeat such a study on a larger scale. Repeating this approach on a larger sample size, in a wild population or in a controlled captive population, may give a clearer indication whether there is indeed scope for the use of individual preference functions to estimate perceived mate attractiveness.

\section{Differential allocation}

Choosers are expected to change their own care level depending on their choice for a mate (Burley 1988; Gil et al. 1999; Sheldon 2000; Bolund et al. 2009). Depending on different factors such as how mate quality benefits reproductive success, the choosers own physical state and the future prospects of reproduction or availability of potential mates, individuals can invest more or less into reproduction. In chapters 4 and 5 I studied whether there might be pre-hatching differential allocation by females depending on how attractive they perceived their mate to be.

In chapter 5 I found that females invested differentially in egg weight depending on their mates' personality: females paired with faster males laid heavier eggs. Since fast males were found to provision less, females with fast males may have invested more in their eggs as compensatory investment (Hollander et al. 2008; Mutzel et al. 2013). However, since fast females also provision less, a compensation in egg weight could also be beneficial for fast females themselves, but this was not found by this study. Alternatively, if fast males hold better territories (Both et al. 2005; Scales et al. 2013) females could also have increased their investment in egg weight when they are paired with these fast males.

Where most studies test the effects of male traits on female pre-hatching investment, here I also studied how females responded to how attractive they perceived their mate to be. If 
females differ in their preferences for male traits, their responses to these traits may also differ depending on those preferences. I expected females to respond to the IMA with differential allocation, with either a higher or a lower investment. Females however, did not vary their investment depending on their IMA. Alternatively, a differential investment by the females could also have depended on the IMA for the different traits separately (heterozygosity, yellowness, stripe size and body condition). Females may respond differently to the different traits depending on which benefit compatibility for these traits may give them. Thus, for some traits females may increase or decrease their reproductive investment with increasing IMA, while for other traits the IMA might not have made a difference. However, also the trait specific IMA scores were not correlated with female pre-hatching investment. Possibly females still invested differentially, but only in response to the actual male trait values and the expected benefits related to these traits, rather than to how attractive they perceived their mates to be. Alternatively, females may invest differentially after hatching or with types of investment that we did not measure. For instance through the deposit of carotenoids or androgens in the eggs (Kingma et al. 2009; Marri and Richner 2014).

Nevertheless, since differential female allocation can compensate for or increase the beneficial effects of male traits, certain male traits may correlate with an increased reproductive success. Although this may suggest effects of male quality or attractiveness, the benefits may actually come from the females' investment. Therefore, in studying mate choice and mate compatibility it is important to be aware of these potential effects (Hettyey et al. 2010).

\section{Consequences for sexual selection}

\section{Solution for the Lek paradox?}

Individual differences in preferences for a mate, rather than population-wide preferences, could be a mechanism that maintains genetic variation in traits and preferences (Neff and Pitcher 2005; Kotiaho et al. 2008; Neff and Pitcher 2009). Preferences for compatibility, such as the ones I found here, can reduce directional good-genes preferences, and thereby maintain the genetic variation in traits and in preferences (Colegrave et al. 2002). If one chooser maximises its fitness by mating with one type of courter and another chooser achieves this with another type of courter, this could work through a kind of epistasis: some preference-trait combinations have higher fitness than others. While these epistatic effects make it difficult to predict responses to selection, it may play a larger role than we commonly assume (Rosenthal 2017). Additionally, variation in mate preferences can decrease variance in male mating success - and with it the strength of sexual selection 
(Brooks and Endler 2001; Dougherty and Shuker 2014). Modelling studies incorporating the potential for preferences for compatibility may shed light on how these preferences may evolve and what the consequences are for sexual selection.

Additionally, the Lek paradox posits that females only gain additive genetic benefits from choosing attractive mates. However, in socially monogamous species with biparental care direct benefits of finding a suitable mate also play a large role, if not a larger role in reproductive success. Consequently, when direct benefits of mate choice are higher, preferences for direct benefits may also evolve more easily than preferences for indirect benefits (Kuijper et al. 2012; Rosenthal 2017). This also suggests that if direct benefits are gained from compatibility between pair mates (Ihle et al. 2015; chapter 3 ) preferences for (behavioural) compatibility are also likely to evolve.

\section{Future research and promising research trajectories}

In this thesis I described how mate preferences may have evolved to gain direct or indirect benefits from pairing with a compatible partner. In the subsequent breeding season, if individuals paired up with a mate according to those preferences, these compatible pairs were better at rearing offspring (chapter 3). However, surprisingly, such preferences are often not reflected in the mating patterns, despite the associated benefits (Booksmythe et al. 2011; Duval and Kapoor 2015; chapter 3 and 4). This discrepancy may be caused by the fact that, although birds have preferences for certain traits in a mate, in real life these preferences are constrained by the ecological context and by social factors like availability of potential mates, competition and sampling investment (Jennions and Petrie 1997; Wagner 1998; Widemo and Sæther 1999). Thus, individuals have to decide upon a mate, whilst being affected by internal and external factors. Therefore, a very interesting direction for future research would be to study how mate preferences are translated into choice. Knowledge of how individuals sample the available options and weigh the attractiveness of each potential partner combined with information on the life history of a species could give a better understanding of the process sexual selection and the role that preferences and choice play.

Additionally, although most theoretical models assume one choosy sex (Kokko et al. 2003), in most mating systems pair formation is actually a mutual decision (Rosenthal 2017). Especially in species with biparental care, both males and females benefit from finding a compatible mate, which makes mutual mate choice likely (Kokko and Johnstone 2002). In this thesis, I found that males and females did not differ in their preferences for genetic traits in a mate (chapter 3 ). This shows that both males and females have preferences and considering that both would benefit from finding a compatible mate one could argue that 
both sexes should benefit from being choosy in finding a mate. In the literature however, the effects of mutual mate choice, compatibility and social environment are currently often overlooked. Thus, are males also selective in finding a mate? And how do mutual decisions and social context shape the realised outcomes?

In chapter 4 I studied how attractiveness may affect reproductive success. While studies performed in captivity often find reproductive benefits of pairing with attractive mates (Koeninger, Ryan and Altmann 2001; Drickamer et al. 2003; Gowaty et al. 2003; Anderson et al. 2007; Ihle et al. 2015), the effects of individual mate attractiveness have, to my knowledge, not been studied in the field. Despite the fact that, with this limited sample size I did not find IMA to affect reproductive success, this may be a very promising method to study the effects of pair compatibility on reproductive success. Repeating such an approach on a larger sample size, in a wild population or in a controlled captive population, may give a clearer indication whether there is scope for the use of individual preference function to estimate perceived mate attractiveness.

And finally, we currently live in a world that is challenged by human induced environmental changes that have the potential to affect the process of sexual selection (Tuomainen and Candolin 2011). For instance human-induced water pollution is known to affect the turbidity in waters, and with it the visibility of sexual signals (Järvenpää and Lindström 2004; Engström-Öst and Candolin 2007; Maan et al. 2009; Sundin et al. 2010). Moreover, pollution from endocrine disrupting chemicals can change courtship and parental care levels in sand gobies (Pomatoschistus minutus; Saaristo et al. 2009; Saaristo et al. 2010) and pesticides can impair pheromonal communication essential for mate choice behaviour in red spotted newts (Notophthalmus viridescens; Park et al. 2001). Do individuals use multiple signals to select a mate? And could they use other non-disturbed signals as replacing signals for quality (Candolin 2003; Engström-Öst and Candolin 2007)? Do these changes in mate selection affect choice for compatible mates, and with it, does it affect reproductive success? To fully understand what the impact and consequences of these human induced changes are on sexual selection, and subsequently how this may affect the fitness of the species, we thus need a better insight into how the different components in the process of mate choice are affected.

\section{Conclusion}

To conclude, in this thesis I have investigated individual differences in mate preferences and the role of compatibility in mate preferences, mate choice and reproductive success. Additionally, I have revealed how individual great tits assess potential partners when given the opportunity to compare the available options. Here I have found that 
preferences for a compatible, rather than universally attractive, partner may have evolved to gain direct or indirect benefits from pairing. While the measured mate preferences were not reflected in the mating patterns, which may be due to the many constraints regarding mate choice, those individuals that did find a mate according to their preferences had a higher reproductive success. Moreover, these results illustrate that we have to consider preference, choice and the fitness benefits related to these preferences to increase our understanding of sexual selection and the selective pressures leading to the evolution of these mate preferences. 



\section{References}

Abràmoff MD, Magalhães PJ, Ram SJ. 2004. Image processing with imageJ. Biophotonics Int. 11:36-41. doi:10.1117/1.3589100.

Acevedo-Whitehouse K, Greig D, Amos W. 2002. Disease susceptibility in California sea lions. Nature 11264:35. doi:10.1038/422035a.

Aeschlimann PB, Häberli MA, Reusch TBH, Boehm T, Milinski · M. 2003. Female sticklebacks Gasterosteus aculeatus use self-reference to optimize MHC allele number during mate selection. Behav Ecol Sociobiol 54:119-126. doi:10.1007/s00265-003-0611-6.

Alatalo R V., Lundberg A, Glynn C. 1986. Female pied flycatchers choose territory quality and not male characteristics. Nature 323:152-153. doi:10.1038/323152a0.

Alho JS, Välimäki K, Merilä J. 2010. Rhh: An R extension for estimating multilocus heterozygosity and heterozygosity-heterozygosity correlation. Mol. Ecol. Resour. 10:720-722. doi:10.1111/j.1755-0998.2010.02830.x.

Alonzo SH. 2012. Sexual selection favours male parental care, when females can choose. Proc. R. Soc. B Biol. Sci. 279:1784-1790. doi:10.1098/rspb.2011.2237.

Altman DG. 2005. Categorizing Continuous Variables. In: Armitage P, Colton T, editors. Encyclopedia of Biostatistics. Chichester, England: John Wiley \& Sons, Ltd.

Amundsen T. 2000. Why are female birds ornamented? Trends Ecol. Evol. 15:149-155. doi:10.1016/S0169-5347(99)01800-5.

Amundsen T, Forsgren E. 2001. Male mate choice selects for female coloration in a fish. Proc. Natl. Acad. Sci. U. S. A. 98:13155-60. doi:10.1073/pnas.211439298.

Anderson WW, Kim Y-K, Gowaty PA. 2007. Experimental constraints on mate preferences in Drosophila pseudoobscura decrease offspring viability and fitness of mated pairs. Proc. Natl. Acad. Sci. U. S. A. 104:4484-4488. doi:10.1073/pnas.0611152104.

Andersson M. 1994. Sexual selection. Princeton: Princeton Univ. Press (Monographs in behavior and ecology).

Andersson M, Iwasa Y. 1996. Sexual selection. Trends Ecol. Evol. 11:53-58. doi:10.1016/0169-5347(96)81042-1.

Andersson M, Simmons LW. 2006. Sexual selection and mate choice. Trends Ecol. Evol. 21:296-302. doi:10.1016/j.tree.2006.03.015.

Andersson S, Prager M. 2006. Quantification of Coloration. In: Hill GE, McGraw KJ, editors. Bird Coloration: Mechanisms and Measurements. Cambridge (Massachusetts): Harvard University Press. p. 41-89.

Aparicio JM, Ortego J, Cordero PJ. 2006. What should we weigh to estimate heterozygosity, alleles or loci? Mol. Ecol. 15:4659-4665. doi:10.1111/j.1365294X.2006.03111.x.

Arct A, Drobniak SM, Cicho M. 2015. Genetic similarity between mates predicts extrapair paternity - a meta-analysis of bird studies. Behav. Ecol. doi:10.1093/beheco/arv004. 
Ariyomo TO, Watt PJ. 2013. Disassortative mating for boldness decreases reproductive success in the guppy. Behav. Ecol. 24:1320-1326. doi:10.1093/beheco/art070.

Arnold KE, Gilbert L, Gorman HE, Griffiths KJ, Adam A, Nager RG. 2016. Paternal attractiveness and the effects of differential allocation of parental investment. Anim. Behav. 113:69-78. doi:10.1016/j.anbehav.2015.12.013.

Arnqvist G, Rowe L, Krupa JJ, Sih A. 1996. Assortative mating by size: A meta-analysis of mating patterns in water striders. Evol. Ecol. 10:265-284. doi:10.1007/BF01237684.

Bailey NW, Zuk M. 2009. Field crickets change mating preferences using remembered social information. Biol. Lett. 5:449-451. doi:10.1098/rsbl.2009.0112.

Bakker TCM. 1993. Positive genetic correlation between female preference and preferred male ornament in sticklebacks. Nature 363:255-257. doi:10.1038/363255a0.

Bakker TCM, Milinski M. 1993. The advantages of being red: sexual selection in the stickleback. Mar. Behav. Physiol. 23:287-300. doi:10.1080/10236249309378870.

Van Balen JH. 1973. A comparative study of the breeding ecology of the great tit Parus major in different habitats. Ardea 61:1-93. doi:10.1560/4LHW-DW74-BQUW-5WM0.

Balloux F, Amos W, Coulson T. 2004. Does heterozygosity estimate inbreeding in real populations? Mol. Ecol. 13:3021-3031. doi:10.1111/j.1365-294X.2004.02318.x.

Barnett CA, Thompson CF, Sakaluk SK. 2012. Aggressiveness, boldness and parental food provisioning in male house wrens (Troglodytes aedon). Ethology 118:984-993. doi:10.1111/j.1439-0310.2012.02092.x.

Barry KL, Holwell GI, Herberstein ME. 2010. Multimodal mate assessment by male praying mantids in a sexually cannibalistic mating system. Anim. Behav. 79:1165-1172. doi:10.1016/j.anbehav.2010.02.025.

Barry KL, Kokko H. 2010. Male mate choice: why sequential choice can make its evolution difficult. Anim. Behav. 80:163-169. doi:10.1016/j.anbehav.2010.04.020.

Bartón K. 2017. MuMIn: Multi-model inference.

Bateman AJ. 1948. Intra-sexual selection in Drosophila. Heredity (Edinb). 2:349-368. doi:10.1038/hdy.1948.21.

Bates D, Maechler M, Bolker BM, Walker S. 2015. lme4: Linear mixed-effects models using Eigen and S4.

Bateson M, Healy SD. 2005. Comparative evaluation and its implications for mate choice. Trends Ecol. Evol. 20:659-64. doi:10.1016/j.tree.2005.08.013.

Bateson P. 1982. Preferences for cousins in Japanese quail. Nature 295:236-237. doi:10.1038/295236a0.

Bateson P. 1983. Optimal outbreeding. In: Bateson P, editor. Mate choice. Cambridge: Cambridge University Press. p. 257-277.

Beatty CD, Franks DW. 2012. Discriminative predation: Simultaneous and sequential encounter experiments. Curr. Zool. 58:649-657. 
Bel-Venner M. C, Dray S, Allainé D, Menu F, Venner S, Allaine D, Menu F, Venner S. 2008. Unexpected male choosiness for mates in a spider. Proc. R. Soc. B Biol. Sci. 275:77-82. doi:10.1098/rspb.2007.1278.

Bell AM, Hankison SJ, Laskowski KL. 2009. The repeatability of behaviour: a metaanalysis. Anim. Behav. 77:771-783. doi:10.1016/j.anbehav.2008.12.022.

Benton TG, Evans MR. 1998. Measuring mate choice using correlation: The effect of female sampling behaviour. Behav. Ecol. Sociobiol. 44:91-98. doi:10.1007/s002650050520.

Benus RF, Koolhaas JM, van Oortmerssen G. 1987. Individual differences in behavioural reaction to a changing environment in mice and rats. Behaviour 100:105-122.

Bluhm CK, Gowaty PA. 2004. Reproductive compensation for offspring viability deficits by female mallards, Anas platyrhynchos. Anim. Behav. 68:985-992. doi:10.1016/j.anbehav.2004.01.012.

Bolund E, Schielzeth H, Forstmeier W. 2009. Compensatory investment in zebra finches: females lay larger eggs when paired to sexually unattractive males. Proc. R. Soc. B Biol. Sci. 276:707-715. doi:10.1098/rspb.2008.1251.

Bonneaud C, Chastel O, Federici P, Westerdahl H, Sorci G. 2006. Complex Mhc-based mate choice in a wild passerine. Proc. R. Soc. B Biol. Sci. 273:1111-1116. doi:10.1098/rspb.2005.3325.

Booksmythe I, Jennions MD, Backwell PRY. 2011. Male fiddler crabs prefer conspecific females during simultaneous, but not sequential, mate choice. Anim. Behav. 81:775-778. doi:10.1016/j.anbehav.2011.01.009.

Borgia GN. 1979. Sexual selection and the evolution of mating systems. In: Blum MS, Blum NA, editors. Sexual Selection and Reproductive Competition in Insects. New York: Academic Press New York. p. 19-80.

Both C, Dingemanse NJ, Drent PJ, Tinbergen JM. 2005. Pairs of extreme avian personalities have highest reproductive success. J. Anim. Ecol. 74:667-674. doi:10.1111/j.13652656.2005.00962.x.

Brandt LRSE, Ludwar BC, Greenfield MD. 2005. Co-occurrence of preference functions and acceptance thresholds in female choice: Mate discrimination in the lesser wax moth. Ethology 111:609-625. doi:10.1111/j.1439-0310.2005.01085.x.

Brinkhof MWG, Heeb P, Kolliker M, Richner H. 1999. Immunocompetence of nestling great tits in relation to rearing environment and parentage. Proc. R. Soc. B Biol. Sci. 266:2315-2322. doi:10.1098/rspb.1999.0925.

Bro-Jørgensen J. 2002. Overt female mate competition and preference for central males in a lekking antelope. Proc. Natl. Acad. Sci. U. S. A. 99:9290-9293. doi:10.1073/pnas.142125899.

Brommer JE. 2000. The evolution of fitness in life - history theory. Biol. Rev 75:377-404. doi:10.1111/j.1469-185X.2000.tb00049.x.

Brooks RC, Endler JA. 2001. Female guppies agree to differ: phenotypic and genetic variation in mate-choice behavior and the consequences for sexual selection. Evolution (N. Y). 55:1644-55. 
Brouwer L, Van De Pol M, Atema E, Cockburn A. 2011. Strategic promiscuity helps avoid inbreeding at multiple levels in a cooperative breeder where both sexes are philopatric. Mol. Ecol.:4796-4807. doi:10.1111/j.1365-294X.2011.05325.x.

Budaev S V., Zworykin DD, Mochek AD. 1999. Individual differences in parental care and behaviour profile in the convict cichlid: a correlation study. Anim. Behav. 58:195-202. doi:10.1006/anbe.1999.1124.

Budden AE, Wright J. 2008. Effects of feeding frequency on nestling. :234-241.

Burke EJ, Murphy CG. 2007. How female barking treefrogs, Hyla gratiosa, use multiple call characteristics to select a mate. Anim. Behav. 74:1463-1472. doi:10.1016/j.anbehav.2007.02.017.

Burley N. 1986. Sexual selection for aesthetic traits in species with biparental care. Am. Nat. 127:415-445. doi:10.1086/284493.

Burley N. 1988. The differential-allocation hypothesis: An experimental test. Am. Nat. 132:611-628.

Burley NT, Hamedani E, Symanski C. 2018. Mate choice decision rules: Trait synergisms and preference shifts. Ecol. Evol.:1-15. doi:10.1002/ece3.3831.

Burnham KP, Anderson DR. 2002. Model selection and multimodal inference. Second. New York, NY: Springer Science+Business Media, LLC.

Byers JA, Waits L. 2006. Good genes sexual selection in nature. Proc. Natl. Acad. Sci. 103:16343-16345. doi:10.1073/pnas.0608184103.

Byers JA, Wiseman PA, Jones L, Roffe TJ. 2005. A large cost of female mate sampling in pronghorn. Am. Nat. 166:661-668. doi:10.1086/497401.

Cameron E, Day T, Rowe L. 2003. Sexual conflic and indirect benefits. J. Evol. Biol. 16:10551060. doi:10.1046/j.1420-9101.2003.00584.x.

Candolin U. 2003. The use of multiple cues in mate choice. Biol. Rev. Camb. Philos. Soc. 78:575-95. doi:10.1017/S1464793103006158.

Van de Casteele T, Galbusera P, Schenck T, Matthysen E. 2003. Seasonal and reproductive consequences of inbreeding in the great tit Parus major. Behav. Ecol. 14:165-174. doi:10.1093/beheco/14.2.165.

Castellano S, Cadeddu G, Cermelli P. 2012. Computational mate choice: Theory and empirical evidence. Behav. Processes 90:261-277. doi:10.1016/j.beproc.2012.02.010.

Chaine AS, Lyon BE. 2008. Adaptive plasticity in female mate choice dampens sexual selection on male ornaments in the lark bunting. Science (80). 319:459-62. doi:10.1126/science.1149167.

Chang CC, Teo HY, Norma-Rashid Y, Li D. 2017. Predator personality and prey behavioural predictability jointly determine foraging performance. Sci. Rep. 7:1-8. doi:10.1038/srep40734.

Chapman JR, Nakagawa S, Coltman DW, Slate J, Sheldon BC. 2009. A quantitative review of heterozygosity-fitness correlations in animal populations. Mol. Ecol. 18:2746-2765. doi:10.1111/j.1365-294X.2009.04247.x. 
Charlesworth D, Charlesworth B. 1987. Inbreeding depression and its evolutionary consequences. Annu. Rev. Ecol. Syst. Vol. 18:237-268. doi:10.1146/annurev.es.18.110187.001321.

Class B, Kluen E, Brommer JE. 2014. Evolutionary quantitative genetics of behavioral responses to handling in a wild passerine. Ecol. Evol. 4:427-440. doi:10.1002/ece3.945.

Clayton NS. 1990. Mate choice and pair formation in Timor and Australian Mainland zebra finches. Anim. Behav. 39:474-480. doi:10.1016/S0003-3472(05)80411-7.

Cohen LB, Dearborn DC. 2004. Great frigatebirds, Fregata minor, choose mates that are genetically similar. Anim. Behav. 68:1229-1236. doi:10.1016/j.anbehav.2003.12.021.

Colegrave N, Kotiaho JS, Tomkins JL. 2002. Mate choice or polyandry : reconciling genetic compatibility and good genes sexual selection. :911-917.

Coltman DW, Bowen WD, Wright JM. 1998. Birth weight and neonatal survival of harbour seal pups are positively correlated with genetic variation measured by microsatellites. Proc. R. Soc. B Biol. Sci. 265:803-809.

Costantini D, Casagrande S, Di Lieto G, Fanfani A, Dell'Omo G. 2005. Consistent differences in feeding habits between neighbouring breeding kestrels. Behaviour 142:1403-1415. doi:10.1163/156853905774539409.

Cotton S, Rogers DW, Small J, Pomiankowski A, Fowler K. 2006. Variation in preference for a male ornament is positively associated with female eyespan in the stalk-eyed fly Diasemopsis meigenii. Proc. R. Soc. B Biol. Sci. 273:1287-92. doi:10.1098/rspb.2005.3449.

Cotton S, Small J, Pomiankowski A. 2006. Sexual selection and condition-dependent mate preferences. Curr. Biol. 16:R755-65. doi:10.1016/j.cub.2006.08.022.

Coulon A. 2010. Genhet: An easy-to-use R function to estimate individual heterozygosity. Mol. Ecol. Resour. 10:167-169. doi:10.1111/j.1755-0998.2009.02731.x.

Culina A. 2014. With or without you - pair fidelity and divorce in monogamous birds. PhD thesis, University of Oxford.

Cunningham EJ, Russell AF. 2000. Egg investment is influenced by male attractiveness in the mallard. Nature 404:74-7. doi:10.1038/35003565.

Dale S, Rinden H, Slagsvold T. 1992. Competition for a mate restricts mate search of female pied flycatchers. Behav. Ecol. Sociobiol. 30:165-176. doi:10.1007/BF00166699.

Dale S, Slagsvold T. 1996. Mate choice on multiple cues, decision rules and sampling strategies in female pied flycatchers. Behaviour 133:903-944.

Danchin E, Cézilly F. 2008. Sexual selection: another evolutionary process. In: Behavioural Ecology. Oxford University Press, USA. p. 363-426.

Darwin C. 1871. The Descent of Man, and Selection in Relation to Sex. London: John Murray.

David M, Cézilly F. 2011. Personality may confound common measures of mate-choice. PLoS One 6:e24778. doi:10.1371/journal.pone.0024778.

David M, Cézilly F, Giraldeau L-A. 2011. Personality affects zebra finch feeding success in a producer-scrounger game. Anim. Behav. 82:61-67. doi:10.1016/j.anbehav.2011.03.025. 
David M, Pinxten R, Martens T, Eens M. 2015. Exploration behavior and parental effort in wild great tits: partners matter. Behav. Ecol. Sociobiol. 69:1085-1095. doi:10.1007/s00265015-1921-1.

David P, Pujol B, Viard F, Castella V, Goudet J. 2007. Reliable selfing rate estimates from imperfect population genetic data. Mol. Ecol. 16:2474-2487. doi:10.1111/j.1365294X.2007.03330.x.

Davies NB. 1977. Prey selection and the search strategy of the Spotted Flycatcher (Muscipcapa striata): A field study on optimal foraging. Anim. Behav. 25:1016-1033. doi:http://dx.doi.org/10.1016/0003-3472(77)90053-7.

Dechaume-Moncharmont F-X, Brom T, Cézilly F. 2016. Opportunity costs resulting from scramble competition within the choosy sex severely impair mate choosiness. Anim. Behav. 114:249-260. doi:10.1016/j.anbehav.2016.02.019.

Dechaume-Moncharmont F-X, Cornuau JH, Keddar I, Ihle M, Motreuil S, Cézilly F. 2011. Rapid assessment of female preference for male size predicts subsequent choice of spawning partner in a socially monogamous cichlid fish. Comptes Rendus - Biol. 334:906-910. doi:10.1016/j.crvi.2011.08.004.

Dechaume-Moncharmont F-X, Freychet M, Motreuil S, Cézilly F. 2013. Female mate choice in convict cichlids is transitive and consistent with a self-referent directional preference. Front. Zool. 10:69. doi:10.1186/1742-9994-10-69.

Dhondt AA, Adriaensen F, Plompen W. 1996. Between- and within-population variation in mate fidelity in the Great Tit. In: Partnerships in birds; The study of monogamy:235248.

Dingemanse NJ. 2004. The relation between dominance and exploratory behavior is context-dependent in wild great tits. Behav. Ecol. 15:1023-1030. doi:10.1093/beheco/arh115.

Dingemanse NJ, Both C, Drent PJ, van Oers K, van Noordwijk AJ. 2002. Repeatability and heritability of exploratory behaviour in great tits from the wild. Anim. Behav. 64:929938. doi:10.1006/anbe.2002.2006.

Dingemanse NJ, Both C, Drent PJ, Tinbergen JM. 2004. Fitness consequences of avian personalities in a fluctuating environment. Proc. R. Soc. B Biol. Sci. 271:847-52. doi:10.1098/rspb.2004.2680.

Dingemanse NJ, Reale D. 2013. Natural Selection and Animal Personality. Behaviour 142:1159-1184.

van Doorn GS, Weissing FJ. 2004. The evolution of female preferences for multiple indicators of quality. Am. Nat. 164:173-86. doi:10.1086/422203.

Dougherty LR, Shuker DM. 2014. The effect of experimental design on the measurement of mate choice: a meta-analysis. Behav. Ecol. 00:1-9. doi:10.1093/beheco/aru125.

Drent PJ. 1983. The functional ethology of territoriality in the great tit (Parus major L.). PhD thesis, University of Groningen.

Drent PJ, Marchetti C. 1999. Individuality, exploration and foraging in hand raised juvenile great tits. In: Adams NJ, Slotow RH, editors. Proceedings of the 22nd 
International Ornithological Congress, Durban. Johannesburg: BirdLife South Africa. p. 896-914.

Drickamer LC, Gowaty PA, Wagner DM. 2003. Free mutual mate preferences in house mice affect reproductive success and offspring performance. Anim. Behav. 65:105-114. doi:10.1006/anbe.2002.2027.

Duval EH, Kapoor JA. 2015. Causes and consequences of variation in female mate search investment in a lekking bird. Behav. Ecol. 26:1537-1547. doi:10.1093/beheco/arv110.

Duval EH, Kempenaers B. 2008. Sexual selection in a lekking bird: the relative opportunity for selection by female choice and male competition. Proc. R. Soc. B Biol. Sci. 275:19952003. doi:10.1098/rspb.2008.0151.

Edly-Wright C, Schwagmeyer PL, Parker PG, Mock DW. 2007. Genetic similarity of mates, offspring health and extrapair fertilization in house sparrows. Anim. Behav. 73:367-378. doi:10.1016/j.anbehav.2006.08.008.

Edward DA. 2015. The description of mate choice. Behav. Ecol. 00:301-310. doi:10.1093/beheco/aru142.

Edward DA, Chapman T. 2011. The evolution and significance of male mate choice. Trends Ecol. Evol. 26:647-54. doi:10.1016/j.tree.2011.07.012.

Von Engelhardt N, Groothuis TGG. 2011. Maternal Hormones in avian eggs. In: Lopez $\mathrm{KH}$, Norris DO, editors. Hormones and reproduction of vertebrates. San Diego, CA: Academic Press. p. 91-127.

Engqvist L, Sauer KP. 2001. Strategic male mating effort and cryptic male choice in a scorpionfly. Proc. R. Soc. B Biol. Sci. 268:729-735. doi:10.1098/rspb.2000.1423.

Engström-Öst J, Candolin U. 2007. Human-induced water turbidity alters selection on sexual displays in sticklebacks. Behav. Ecol. 18:393-398. doi:10.1093/beheco/arl097.

Eshel I, Volovik I, Sansone E. 2000. On Fisher-Zahavi's handicapped sexy son. Evol. Ecol. Res. 2:509-523.

Evans SR, Hinks AE, Wilkin TA, Sheldon BC. 2010. Age, sex and beauty: methodological dependence of age- and sex-dichromatism in the great tit Parus major. Biol. J. Linn. Soc. 101:777-796. doi:10.1111/j.1095-8312.2010.01548.x.

Fawcett TW, Johnstone RA. 2003. Optimal assessment of multiple cues. Proc. R. Soc. B Biol. Sci. 270:1637-1643. doi:10.1098/rspb.2003.2328.

Ferrer ES, García-navas V, Bueno-Enciso J, Sanz JJ, Ortego J. 2015. Multiple sexual ornaments signal heterozygosity in male blue tits. Biol. J. Linn. Soc. 115:362-375. doi:10.1111/bij.12513.

Figuerola J, Senar JC. 2000. Measurement of plumage badges: an evaluation of methods used in the Great Tit Parus major. Ibis (Lond. 1859). 142:482-484. doi:10.1111/j.1474919X.2000.tb04445.x.

Fisher RA. 1930. The Genetical Theory of Natural Selection: A Complete Variorum Edition.

Foerster K, Delhey K, Johnsen A, Lifjeld JT, Kempenaers B. 2003. Females increase offspring heterozygosity and fitness through extra-pair matings. Nature 425:714-717. doi:10.1038/nature01969. 
Foerster K, Valcu M, Johnsen A, Kempenaers B. 2006. A spatial genetic structure and effects of relatedness on mate choice in a wild bird population. Mol. Ecol. 15:4555-4567. doi:10.1111/j.1365-294X.2006.03091.x.

Forstmeier W, Birkhead TR. 2004. Repeatability of mate choice in the zebra finch: consistency within and between females. Anim. Behav. 68:1017-1028. doi:10.1016/j.anbehav.2004.02.007.

Frasier TR. 2008. STORM: Software for testing hypotheses of relatedness and mating patterns. Mol. Ecol. Resour. 8:1263-1266. doi:10.1111/j.1755-0998.2008.02358.x.

Freeman-Gallant CR, Meguerdichian M, Wheelwright NT, Sollecito S V. 2003. Social pairing and female mating fidelity predicted by restriction fragment length polymorphism similarity at the major histocompatibility complex in a songbird. Mol. Ecol. 12:3077-3083. doi:10.1046/j.1365-294X.2003.01968.x.

Freeman-Gallant CR, Wheelwright NT, Meiklejohn KE, Sollecito S V. 2006. Genetic similarity, extrapair paternity, and offspring quality in Savannah sparrows (Passerculus sandwichensis). Behav. Ecol. 17:952-958. doi:10.1093/beheco/ar1031.

Gabriel PO, Black JM. 2012. Behavioural syndromes, partner compatibility and reproductive performance in Steller's jays. Ethology 118:76-86. doi:10.1111/j.14390310.2011.01990.x.

Galeotti P, Sacchi R, Rosa DP, Fasola M. 2005. Female preference for fast-rate, high-pitched calls in Hermann's tortoises Testudo hermanni. Behav. Ecol. 16:301-308. doi:10.1093/beheco/arh165.

García-navas V, Ferrer ES, Sanz JJ. 2012. Plumage yellowness predicts foraging ability in the blue tit Cyanistes caeruleus. :418-429.

García-navas V, Ortego J, Sanz JJ. 2009. Heterozygosity-based assortative mating in blue tits (Cyanistes caeruleus): implications for the evolution of mate choice. Proc. R. Soc. B Biol. Sci. 276:2931-40. doi:10.1098/rspb.2009.0417.

Gerlach G, Lysiak N. 2006. Kin recognition and inbreeding avoidance in zebrafish, Danio rerio, is based on phenotype matching. Anim. Behav. 71:1371-1377. doi:10.1016/j.anbehav.2005.10.010.

Gil D, Graves J, Hazon N, Wells A. 1999. Male attractiveness and differential testosterone investment in zebra finch eggs. Science (80). 286:126-129. doi:10.1126/science.286.5437.126.

Godin J-GJ, Briggs SE. 1996. Female mate choice under predation risk in the guppy. Anim. Behav 51:117-130. doi:10.1006/anbe.1996.0010.

Godin J-GJ, Dugatkin LA. 1995. Variability and repeatability of female mating in the guppy. Anim. Behav. 49:1427-1433. doi:10.1016/0003-3472(95)90063-2.

Gosler A. 1993. The great tit. London: Hamlyn.

Gowaty PA, Anderson WW, Bluhm CK, Drickamer LC, Kim Y-K, Moore AJ. 2007. The hypothesis of reproductive compensation and its assumptions about mate preferences and offspring viability. Proc. Natl. Acad. Sci. U. S. A. 104:15023-7. doi:10.1073/pnas.0706622104. 
Gowaty PA, Drickamer LC, Schmid-Holmes S. 2003. Male house mice produce fewer offspring with lower viability and poorer performance when mated with females they do not prefer. Anim. Behav. 65:95-103. doi:10.1006/anbe.2002.2026.

Greeff JM, Jansen van Vuuren GJ, Kryger P, Moore JC. 2009. Outbreeding and possibly inbreeding depression in a pollinating fig wasp with a mixed mating system. Heredity (Edinb). 102:349-356. doi:10.1038/hdy.2009.2.

Grieco F. 2001. Short-term regulation of food-provisioning rate and effect on prey size in blue tits, Parus caeruleus. Anim. Behav. 62:107-116. doi:10.1006/anbe.2001.1736.

Grieco F. 2002. Time constraint on food choice in provisioning blue tits, Parus caeruleus: the relationship between feeding rate and prey size. Anim. Behav. 64:517-526. doi:10.1006/anbe.2002.3073.

Grodzinski U, Hauber ME, Lotem A. 2009. The role of feeding regularity and nestling digestive efficiency in parent-offspring communication: An experimental test. Funct. Ecol. 23:569-577. doi:10.1111/j.1365-2435.2008.01536.x.

Groothuis TGG, Carere C. 2005. Avian personalities: characterization and epigenesis. Neurosci. Biobehav. Rev. 29:137-50. doi:10.1016/j.neubiorev.2004.06.010.

Haaland TR, Wright J, Kuijper B, Ratikainen II. 2017. Differential Allocation Revisited: When Should Mate Quality Affect Parental Investment? Am. Nat. 190:000-000. doi:10.1086/693484.

Hadfield JD, Burgess MD, Lord A, Phillimore AB, Clegg SM, Owens IPF. 2006. Direct versus indirect sexual selection: genetic basis of colour, size and recruitment in a wild bird. Proc. R. Soc. B Biol. Sci. 273:1347-1353. doi:10.1098/rspb.2005.3459.

Hamilton W, Zuk M. 1982. Heritable true fitness and bright birds: a role for parasites? Science (80-. ). 218:384-387. doi:10.1126/science.7123238.

Hansson B, Jack L, Christians JK, Pemberton JM, Åkesson M, Westerdahl H, Bensch S, Hasselquist D. 2007. No evidence for inbreeding avoidance in a great reed warbler population. Behav. Ecol. 18:157-164. doi:10.1093/beheco/arl062.

Hansson B, Westerberg L. 2002. On the correlation between heterozygocity and fitness in natural populations. Mol. Ecol. 11:2467-2474.

Harris MR, Siefferman L. 2014. Interspecific competition influences fitness benefits of assortative mating for territorial aggression in Eastern Bluebirds (Sialia sialis). PLoS One 9:1-6. doi:10.1371/journal.pone.0088668.

Harris WE, Uller T. 2009. Reproductive investment when mate quality varies: differential allocation versus reproductive compensation. Philos. Trans. R. Soc. B Biol. Sci. 364:10391048. doi:10.1098/rstb.2008.0299.

Hawley DM, Sydenstricker K V, Kollias G V, Dhondt A a. 2005. Genetic diversity predicts pathogen resistance and cell-mediated immunocompetence in house finches. Biol. Lett. 1:326-329. doi:10.1098/rsbl.2005.0303.

Hebets EA. 2003. Subadult experience influences adult mate choice in an arthropod: Exposed female wolf spiders prefer males of a familiar phenotype. Proc. Natl. Acad. Sci. 100:13390-13395. doi:10.1073/pnas.2333262100. 
Hegyi G, Szigeti B, Török J, Eens M. 2007. Melanin, carotenoid and structural plumage ornaments: information content and role in great tits Parus major. J. Avian Biol. 38:698708. doi:10.1111/j.2007.0908-8857.04075.x.

Hettyey A, Hegyi G, Puurtinen M, Hoi H, Török J, Penn DJ. 2010. Mate choice for genetic benefits: Time to put the pieces together. Ethology 116:1-9. doi:10.1111/j.14390310.2009.01704.x.

Hill GE. 1990. Female house finches prefer colourful males: sexual selection for a condition-dependent trait. Anim. Behav. 40:563-572. doi:10.1016/S0003-3472(05)80537-8.

Hinde CA. 2005. Reproductive strategies in the great tit (Parus major). PhD thesis, University of Cambridge.

Hinde CA. 2006. Negotiation over offspring care? - A positive response to partnerprovisioning rate in great tits. Behav. Ecol. 17:6-12. doi:10.1093/beheco/ari092.

Hinde CA, Buchanan KL, Kilner RM. 2009. Prenatal environmental effects match offspring begging to parental provisioning. Proc. R. Soc. B Biol. Sci. 276:2787-2794. doi:10.1098/rspb.2009.0375.

Hinde CA, Kilner RM. 2007. Negotiations within the family over the supply of parental care. Proc. Biol. Sci. 274:53-60. doi:10.1098/rspb.2006.3692.

Hinde RA. 1952. The behaviour of the great tit (Parus Major) and some other related species. Behaviour:1-201.

Hoffman JI, Forcada J, Trathan PN, Amos W. 2007. Female fur seals show active choice for males that are heterozygous and unrelated. Nature 445:912-4. doi:10.1038/nature05558.

Hollander FA, Van Overveld T, Tokka I, Matthysen E. 2008. Personality and nest defence in the great tit (Parus major). Ethology 114:405-412. doi:10.1111/j.1439-0310.2008.01488.x.

Holman L, Kokko H. 2014. Local adaptation and the evolution of female choice. In: Hunt J, Hosken DJ, editors. Genotype-by-Environment Interactions and Sexual Selection. Vol. 10329. Wiley-Blackwell. p. 41-62.

Holveck M-J, Riebel K. 2007. Preferred songs predict preferred males: consistency and repeatability of zebra finch females across three test contexts. Anim. Behav. 74:297-309. doi:10.1016/j.anbehav.2006.08.016.

Holveck M-J, Riebel K. 2010. Low-quality females prefer low-quality males when choosing a mate. Proc. R. Soc. B Biol. Sci. 277:153-60. doi:10.1098/rspb.2009.1222.

Horváthová T, Nakagawa S, Uller T. 2012. Strategic female reproductive investment in response to male attractiveness in birds. Proc. R. Soc. B Biol. Sci. 279:163-70. doi:10.1098/rspb.2011.0663.

Houston AI, McNamara JM. 1999. Models of adaptive behaviour: An approach based on state. Cambridge: Cambridge University Press.

Ihle M, Kempenaers B, Forstmeier W. 2015. Fitness benefits of mate choice for compatibility in a socially monogamous species. PLoS Biol. 13:1-21. doi:10.1371/journal.pbio.1002248.

Iwasa Y, Pomiankowski A, Nee S. 1991. The Evolution of Costly Mate Preferences II . The ' Handicap' Principle. Evolution (N. Y). 45:1431-1442. 
Jamieson IG, Taylor SS, Tracy LN, Kokko H, Armstrong DP. 2009. Why some species of birds do not avoid inbreeding: Insights from New Zealand robins and saddlebacks. Behav. Ecol. 20:575-584. doi:10.1093/beheco/arp034.

Janetos AC. 1980. Strategies of female mate choice: a theoretical analysis. Behav. Ecol. Sociobiol. 7:107-112.

Järvenpää M, Lindström K. 2004. Water turbidity by algal blooms causes mating system breakdown in a shallow-water fish, the sand goby Pomatoschistus minutus. Proc. R. Soc. B Biol. Sci. 271:2361-5. doi:10.1098/rspb.2004.2870.

Jennions MD, Petrie M. 1997. Variation in mate choice and mating preferences: Review of causes and consequences. Biol. Rev. Camb. Philos. Soc. 72:283-327. doi:10.1111/j.1469185X.1997.tb00015.x.

Jeswiet SB, Godin J-GJ. 2011. Validation of a method for quantifying male mating preferences in the guppy (Poecilia reticulata). Ethology 117:422-429. doi:10.1111/j.14390310.2011.01891.x.

Jiang Y, Bolnick DI, Kirkpatrick M. 2013. Assortative mating in animals. Am. Nat. 181:E125--38. doi:10.1086/670160.

Johnstone RA. 1995. Honest advertisement of multiple qualities using multiple signals. J. Theor. Biol. 177:87-94. doi:10.1016/S0022-5193(05)80006-2.

Johnstone RA. 1996. Multiple displays in animal communication:'Backup signals' and 'Multiple messages'. Philos. Trans. R. Soc. B Biol. Sci. 351:329-338. doi:10.1098/rstb.1996.0026.

Johnstone RA. 1997. The tactics of mutual mate choice and competitive search. Behav. Ecol. Sociobiol. 40:51-59.

Johnstone RA, Manica A, Fayet AL, Stoddard MC, Rodríguez-Gironés MA, Hinde CA. 2014. Reciprocity and conditional cooperation between great tit parents. Behav. Ecol. 25:216-222. doi:10.1093/beheco/art109.

Jones KM, Monaghan P, Nager RG. 2001. Male mate choice and female fecundity in zebra finches. Anim. Behav. 62:1021-1026. doi:10.1006/anbe.2001.1843.

Kawano KM. 2003. Isolation of polymorphic microsatellite markers in the great tit (Parus major minor). Mol. Ecol. Notes 3:314-315. doi:10.1046/j.1471-8286.2003.00431.x.

Keller LF, Waller DM. 2002. Inbreeding effects in wild populations. Trends Ecol. Evol. 17:230-241. doi:10.1016/S0169-5347(02)02489-8.

Kempenaers B. 2007. Mate choice and genetic quality: A review of the heterozygosity theory. Adv. Study Behav. 37:189-278. doi:10.1016/S0065-3454(07)37005-8.

Kenward MG, Roger JH. 1997. Small Sample Inference for Fixed Effects from Restricted Maximum Likelihood. Biometrics 53:983-997.

Kilmer JT, Fowler-Finn KD, Gray DA, Höbel G, Rebar D, Reichert MS, Rodríguez RL. 2017. Describing mate preference functions and other function-valued traits. J. Evol. Biol.:1-16. doi:10.1111/jeb.13122. 
Kindsvater HK, Alonzo SH. 2014. Females allocate differentially to offspring size and number in response to male effects on female and offspring fitness. Proc. R. Soc. B Biol. Sci. 281:20131981-20131981. doi:10.1098/rspb.2013.1981.

Kingma SA, Komdeur J, Vedder O, Von Engelhardt N, Korsten P, Groothuis TGG. 2009. Manipulation of male attractiveness induces rapid changes in avian maternal yolk androgen deposition. Behav. Ecol. 20:172-179. doi:10.1093/beheco/arn130.

Kirkpatrick M, Barton NH. 1997. The strength of indirect selection on female mating preferences. Proc. Natl. Acad. Sci. U. S. A. 94:1282-6. doi:VL - 94.

Kirkpatrick M, Ryan MJ. 1991. The evolution of mating preferences and the paradox of the lek. Nature 350:33-38. doi:10.1038/350033a0.

Kleven O, Jacobsen F, Robertson RJ, Lifjeld JT. 2005. Extrapair mating between relatives in the barn swallow: a role for kin selection? Biol. Lett. 1:389-92. doi:10.1098/rsbl.2005.0376.

Kluijver HN. 1971. Regulation of numbers in populations of Great Tits (Parus m. major). Proc. Adv. Study Inst. Dyn. Numbers Popul.:507-523.

Ko M, Heeb P, Werner I, Mateman AC, Lessells CM, Richner H. 1999. Offspring sex ratio is related to male body size in the great tit (Parus major). 10:68-72.

Koeninger Ryan K, Altmann J. 2001. Selection for male choice based primarily on mate compatibility in the oldfield mouse, Peromyscus polionotus rhoadsi. Behav. Ecol. Sociobiol. 50:436-440. doi:10.1007/s002650100385.

Kokko H. 1998. Should advertising parental care be honest? Proc. R. Soc. B Biol. Sci. 265:1871-1878. doi:10.1098/rspb.1998.0515.

Kokko H, Brooks RC, Jennions MD, Morley J. 2003. The evolution of mate choice and mating biases. Proc. R. Soc. B Biol. Sci. 270:653-64. doi:10.1098/rspb.2002.2235.

Kokko H, Brooks RC, McNamara JM, Houston AI. 2002. The sexual selection continuum. Proc. R. Soc. B Biol. Sci. 269:1331-40. doi:10.1098/rspb.2002.2020.

Kokko H, Jennions MD, Brooks RC. 2006. Unifying and testing models of sexual selection. Annu. Rev. Ecol. Evol. Syst. 37:43-66. doi:10.2307/annurev.ecolsys.37.091305.30000004.

Kokko H, Johnstone RA. 2002. Why is mutual mate choice not the norm? Operational sex ratios, sex roles and the evolution of sexually dimorphic and monomorphic signalling. Philos. Trans. R. Soc. B Biol. Sci. 357:319-30. doi:10.1098/rstb.2001.0926.

Kokko H, Ots I. 2006. When not to avoid inbreeding. Evolution (N. Y). 60:467-475. doi:10.1111/j.0014-3820.2006.tb01128.x.

Koo J, Jang W, Chung O, Lee W, Lee JK, Jang W, Chung O, Lee W. 2016. The relationships between prey size, nestling age, provisioning rate, and elevation in the Varied Tit Parus varius. Ornithol. Sci. 15:29-36. doi:10.2326/osj.15.29.

Kotiaho JS, LeBas NR, Puurtinen M, Tomkins JL. 2008. On the resolution of the lek paradox. Trends Ecol. Evol. 23:1-3. doi:10.1016/j.tree.2007.09.012.

Kraaijeveld K, Kraaijeveld-Smit FJL, Maan ME. 2011. Sexual selection and speciation: The comparative evidence revisited. Biol. Rev. 86:367-377. doi:10.1111/j.1469185X.2010.00150.x. 
Kraak SBM. 1999. Sexual selection in sticklebacks in the field: correlates of reproductive, mating, and paternal success. Behav. Ecol. 10:696-706. doi:10.1093/beheco/10.6.696.

Kralj-Fišer S, Sanguino Mostajo GA, Preik O, Pekár S, Schneider JM. 2013. Assortative mating by aggressiveness type in orb weaving spiders. Behav. Ecol. 24:824-831. doi:10.1093/beheco/art030.

Krokene C, Lifjeld JT. 2000. Variation in the frequency of extra-pair paternity in birds: A comparison of an island and a mainland population of blue tits. Behaviour 137:13171330. doi:10.1163/156853900501944.

Kuijper B, Pen I, Weissing FJ. 2012. A guide to sexual selection theory. Annu. Rev. Ecol. Evol. Syst. 43:287-311. doi:10.1146/annurev-ecolsys-110411-160245.

Lande R. 1981. Models of speciation by sexual selection on polygenic traits. Proc. Natl. Acad. Sci. U. S. A. 78:3721-3725. doi:10.1073/pnas.78.6.3721.

Lehtonen TK, Lindström K. 2008. Repeatability of mating preferences in the sand goby. Anim. Behav. 75:55-61. doi:10.1016/j.anbehav.2007.04.011.

Lemel J. 1989. Habitat distribution in the Great Tit Parus major in relation to reproductive success, dominance and biometry. Ornis Scand. 20:226-233.

Li CC, Weeks DE, Chakravarti A. 1993. Similarity of DNA fingerprints due to chance and relatedness. Hum. Hered. 43:45-52.

Locatello L, Poli F, Rasotto MB. 2015. Context-dependent evaluation of prospective mates in a fish. Behav. Ecol. Sociobiol. 69:1119-1126. doi:10.1007/s00265-015-1924-y.

Loyau A, Cornuau JH, Clobert J, Danchin É. 2012. Incestuous sisters: mate preference for brothers over unrelated males in Drosophila melanogaster. PLoS One 7:1-6. doi:10.1371/journal.pone.0051293.

Lynch M, Ritland K. 1999. Estimation of pairwise relatedness with molecular markers. Genetics 152:1753-1766.

Maan ME, Seehausen O, Van Alphen JJM. 2009. Female mating preferences and male coloration change with water transparency in a Lake Victoria cichlid fish. Biol. J. Linn. Soc. 99:398-406.

MacDougall-Shackleton EA, Derryberry EP, Foufopoulos J, Dobson AP, Hahn TP. 2005. Parasite-mediated heterozygote advantage in an outbred songbird population. Biol. Lett. 1:105-107. doi:10.1098/rsbl.2004.0264.

Maclaren RD, Rowland WJ. 2016. Differences in female preference for male body size in Poecilia latipinna using simultaneous versus sequential Stimulus Presentation Designs. Behaviour 143:273-292.

Mariette MM, Griffith SC. 2012. Nest visit synchrony is high and correlates with reproductive success in the wild Zebra finch Taeniopygia guttata. J. Avian Biol. 43:131140. doi:10.1111/j.1600-048X.2012.05555.x.

Marri V, Richner H. 2014. Yolk carotenoids increase fledging success in great tit nestlings. Oecologia 176:371-37. doi:10.1007/s00442-014-3051-2. 
Marshall RC, Buchanan KL, Catchpole CK. 2003. Sexual selection and individual genetic diversity in a songbird. Proc. R. Soc. B Biol. Sci. 270:S248-S250. doi:10.1098/rsbl.2003.0081.

Marshall TC, Slate J, Kruuk LEB, Pemberton JM. 1998. Statistical confidence for likelihoodbased paternity. Mol. Ecol. 7:639-655.

Martin-Wintle MS, Shepherdson D, Zhang G, Zhang H, Li D, Zhou X, Li R, Swaisgood RR. 2015. Free mate choice enhances conservation breeding in the endangered giant panda. Nat. Commun. 6:10125. doi:10.1038/ncomms10125.

Mays HL, Albrecht T, Liu M, Hill GE. 2008. Female choice for genetic complementarity in birds: a review. Genetica 134:147-58. doi:10.1007/s10709-007-9219-5.

Mays HL, Hill GE. 2004. Choosing mates: good genes versus genes that are a good fit. Trends Ecol. Evol. 19:554-9. doi:10.1016/j.tree.2004.07.018.

Mazerolle M. 2017. AICcmodavg (v. 2.1.1).

McGlothlin JW, Jawor JM, Ketterson ED. 2007. Natural variation in a testosteronemediated trade-Off between mating effort and parental effort. Am. Nat. 170:864-875. doi:10.1086/522838.

McGraw KJ. 2006. The mechanics of carotenoid coloration in birds. In: Hill G, McGraw K, editors. Bird coloration, Vol. I. Mechanisms and measurements. Boston, MA: Harvard University Press. p. 177-242.

McGuigan K, Van Homrigh A, Blows MW. 2008. Genetic analysis of female preference functions as function-valued traits. Am. Nat. 172:194-202. doi:10.1086/588075.

Mcnamara JM, Gasson CE, Houston AI. 1999. Incorporating rules for responding into evolutionary games. Nature 401:368-371. doi:10.1038/43869.

Milinski M, Bakker TCM. 1990. Female sticklebacks use male coloration in mate choice and hence avoid parasitized males. Nature 344:330-333. doi:10.1038/344330a0.

Milinski M, Bakker TCM. 1992. Costs influence sequential mate choice in sticklebacks, Gasterosteus aculeatus. Proc. R. Soc. B Biol. Sci. 250:229-233. doi:10.1098/rspb.1992.0153.

Mitton JB, Schuett W, Cothran EG, De Fries JC. 1993. Correlation between the individual heterozygosity of parents and their offspring. Heredity 71:59-63. doi:10.1038/hdy.1993.107.

Møller AP, Alatalo R V. 1999. Good-genes effects in sexual selection. Proc. R. Soc. B Biol. Sci. 266:85-91. doi:10.1098/rspb.1999.0607.

Moore AJ, Moore PJ. 1988. Female strategy during mate choice: threshold assessment. Evolution (N. Y). 42:387-390.

Mutzel A, Dingemanse NJ, Araya-Ajoy YG, Kempenaers B. 2013. Parental provisioning behaviour plays a key role in linking personality with reproductive success. Proc. R. Soc. B Biol. Sci. 280:20131019. doi:10.1098/rspb.2013.1019.

Naef-Daenzer L, Naef-Daenzer B, Nager RG. 2000. Prey selection and foraging performance of breeding Great Tits Parus major in relation to food availability. J. Avian Biol. 31:206-214. doi:10.1034/j.1600-048X.2000.310212.x. 
Nakagawa S, Ockendon N, Gillespie DOS, Hatchwell BJ, Burke T. 2007. Does the badge of status influence parental care and investment in house sparrows? An experimental test. Oecologia 153:749-60. doi:10.1007/s00442-007-0765-4.

Neff BD. 2004. Stabilizing selection on genomic divergence in a wild fish population. Proc. Natl. Acad. Sci. U. S. A. 101:2381-2385. doi:10.1073/pnas.0307522100.

Neff BD, Pitcher TE. 2005. Genetic quality and sexual selection: an integrated framework for good genes and compatible genes. Mol. Ecol. 14:19-38. doi:10.1111/j.1365294X.2004.02395.x.

Neff BD, Pitcher TE. 2009. Mate choice for nonadditive genetic benefits and the maintenance of genetic diversity in song sparrows. J. Evol. Biol. 22:424-429. doi:10.1111/j.1420-9101.2008.01646.x.

Nicolaus M, Tinbergen JM, Ubels R, Both C, Dingemanse NJ. 2016. Density fluctuations represent a key process maintaining personality variation in a wild passerine bird. Ecol. Lett. 19:478-486. doi:10.1111/ele.12584.

Norris KJ. 1990. Female choice and the evolution of the conspicuous plumage coloration of monogamous male great tits. Behav. Ecol. Sociobiol. 26. doi:10.1007/BF00171582.

Norris KJ. 1993. Heritable variation in a plumage indicator of viability in male great tits Parus major. Nature 362:537-539. doi:10.1038/362537a0.

van Oers K, Drent PJ, Dingemanse NJ, Kempenaers B. 2008. Personality is associated with extrapair paternity in great tits, Parus major. Anim. Behav. 76:555-563. doi:10.1016/j.anbehav.2008.03.011.

van Oers K, Naguib M. 2013. Avian personality. In: Carere C, Maestripieri D, editors. Animal Personalities: Behavior, Physiology, and Evolution. Chicago: University of Chicago Press. p. 66-95.

Oh KP, Badyaev A V. 2006. Adaptive genetic complementarity in mate choice coexists with selection for elaborate sexual traits. Proc. R. Soc. B Biol. Sci. 273:1913-1919. doi:10.1098/rspb.2006.3528.

Olano-Marin J, Mueller JC, Kempenaers B. 2011. Correlations between heterozygosity and reproductive success in the blue tit (Cyanistes caeruleus): An analysis of inbreeding and single locus effects. Evolution (N. Y). 65:3175-3194. doi:10.1111/j.1558-5646.2011.01369.x.

Ortego J, Calabuig G, Cordero PJ, Aparicio JM. 2007. Egg production and individual genetic diversity in lesser kestrels. Mol. Ecol. 16:2383-2392. doi:10.1111/j.1365294X.2007.03322.x.

Ouyang JQ, van Oers K, Quetting M, Hau M, Oers K Van, Quetting M, Hau M. 2014. Becoming more like your mate: hormonal similarity reduces divorce rates in a wild songbird. Anim. Behav. 98:87-93. doi:10.1016/j.anbehav.2014.09.032.

van Overveld T, Matthysen E. 2010. Personality predicts spatial responses to food manipulations in free-ranging great tits (Parus major). Biol. Lett. 6:187-90. doi:10.1098/rsbl.2009.0764.

van Overveld T, Matthysen E. 2013. Personality and information gathering in free-ranging great tits. PLoS One 8. doi:10.1371/journal.pone.0054199. 
Pagani-Núñez E, Senar JC. 2014. Are colorful males of great tits Parus major better parents? Parental investment is a matter of quality. Acta Oecologica 55:23-28. doi:10.1016/j.actao.2013.11.001.

Park D, Hempleman SC, Propper CR. 2001. Endosulfan exposure disrupts pheromonal systems in the red-spotted newt: A mechanism for subtle effects of environmental chemicals. Environ. Health Perspect. 109:669-673. doi:10.1289/ehp.01109669.

Patrick SC, Browning LE. 2011. Exploration behaviour is not associated with chick provisioning in great tits. PLoS One 6. doi:10.1371/journal.pone.0026383.

Patrick SC, Chapman JR, Dugdale HL, Quinn JL, Sheldon BC. 2012. Promiscuity, paternity and personality in the great tit. Proc. R. Soc. B Biol. Sci. 279:1724-30. doi:10.1098/rspb.2011.1820.

Perrins CM. 1965. Population fluctuations and clutch-size in the Great Tit, Parus major L . J. Anim. Ecol. 34:601-647. doi:10.2307/2453.

Petrie M. 1994. Improved growth and survival of offspring of peacocks with more elaborate trains. Nature 371:598-599. doi:10.1038/371598a0.

Pilakouta N, Smiseth PT. 2017. Female mating preferences for outbred versus inbred males are conditional upon the female's own inbreeding status. Anim. Behav. 123:369-374. doi:10.1016/j.anbehav.2016.11.023.

Pinheiro JC, Bates DM. 2000. Mixed-Effects Models in S and S-PLUS. In: Mixed-Effects Models in S and S-PLUS.

Prokop ZM, Drobniak SM. 2016. Genetic variation in male attractiveness: It is time to see the forest for the trees. Evolution (N. Y). 70:913-921. doi:10.1111/evo.12898.

Prokop ZM, Michalczyk Ł, Drobniak SM, Herdegen M, Radwan J. 2012. Meta-analysis suggests choosy females get sexy sons more than "Good genes." Evolution (N. Y). 66:2665-2673. doi:10.1111/j.1558-5646.2012.01654.x.

Puurtinen M, Ketola T, Kotiaho JS. 2009. The good-genes and compatible-genes benefits of mate choice. Am. Nat. 174:741-752. doi:10.1086/606024.

Queller DC, Goodnight KF. 1989. Estimating relatedness using genetic markers evolution. Evolution (N. Y). 43:258-275.

Quesada J, Senar JC. 2006. Comparing plumage colour measurements obtained directly from live birds and from collected feathers: the case of the great tit Parus major. J. Avian Biol. 37:609-616. doi:10.1111/j.0908-8857.2006.03636.x.

Quesada J, Senar JC. 2009. Cross-fostering experiments to compare carotenoid- and melanin-based plumage traits and long-term parental effects in post-moulted great tits. Behaviour 146:1235-1251. doi:10.1163/156853909X420017.

Qvarnström A, Pärt T, Sheldon BC. 2000. Adaptive plasticity in mate preference linked to differences in reproductive effort. Nature 405:344-7. doi:10.1038/35012605.

R Development Core Team. 2015. R: A language and environment for statistical computing. Version 3.2.3. 
Raihani NJ, Nelson-Flower MJ, Moyes K, Browning LE, Ridley AR. 2010. Synchronous provisioning increases brood survival in cooperatively breeding pied babblers. J. Anim. Ecol. 79:44-52. doi:10.1111/j.1365-2656.2009.01606.x.

Ratikainen II, Kokko H. 2009. Differential allocation and compensation: who deserves the silver spoon? Behav. Ecol. 21:195-200. doi:10.1093/beheco/arp168.

Real L. 1990. Search Theory and Mate Choice . I . Models of Single-Sex Discrimination. Am. Nat. 136:376-405.

Reaney LT. 2009. Female preference for male phenotypic traits in a fiddler crab: do females use absolute or comparative evaluation? Anim. Behav. 77:139-143. doi:10.1016/j.anbehav.2008.09.019.

Reid JM. 2007. Secondary sexual ornamentation and non-additive genetic benefits of female mate choice. Proc. R. Soc. B Biol. Sci. 274:1395-1402. doi:10.1098/rspb.2007.0063.

Reusch TBH, Häberli MA, Aeschlimann PB, Milinski M. 2001. Female sticklebacks count alleles in a strategy of sexual selection explaining MHC polymorphism. Nature 414:300302. doi: $10.1038 / 35104547$.

Richard M, Losdat S, Lecomte J, de Fraipont M, Clobert J. 2009. Optimal level of inbreeding in the common lizard. Proc. R. Soc. B Biol. Sci. 276:2779-2786. doi:10.1098/rspb.2009.0319.

Rijks JM, Hoffman JI, Kuiken T, Osterhaus ADME, Amos W. 2008. Heterozygosity and lungworm burden in harbour seals (Phoca vitulina). Heredity (Edinb). 100:587-593. doi:10.1038/hdy.2008.18.

Robinson SP, Kennington WJ, Simmons LW. 2012. Assortative mating for relatedness in a large naturally occurring population of Drosophila melanogaster. J. Evol. Biol. 25:716-725. doi:10.1111/j.1420-9101.2012.02466.x.

Rodd FH, Hughes KA, Grether GF, Baril CT. 2002. A possible non-sexual origin of mate preference: are male guppies mimicking fruit? Proc. R. Soc. B Biol. Sci. 269:475-481. doi:10.1098/rspb.2001.1891.

Rodríguez RL, Rebar D, Fowler-Finn KD. 2013. The evolution and evolutionary consequences of social plasticity in mate preferences. Anim. Behav. 85:1041-1047. doi:10.1016/j.anbehav.2013.01.006.

Rosenthal GG. 2017. Mate Choice: The evolution of sexual decision making from microbes to humans. 1st ed. Rosenthal GG, editor. Princeton: Princeton University Press.

Rosvall KA. 2010. Do males offset the cost of female aggression? An experimental test in a biparental songbird. Behav. Ecol. 21:161-168. doi:10.1093/beheco/arp167.

Rowe L, Houle D. 1996. The Lek paradox and the capture of genetic variance by condition dependent traits. Proc. R. Soc. B Biol. Sci. 263:1415-1421. doi:10.1098/rspb.1996.0207.

Royle NJ, Lindström J, Metcalfe NB. 2008. Context-dependent mate choice in relation to social composition in green swordtails Xiphophorus helleri. Behav. Ecol. 19:998-1005. doi:10.1093/beheco/arn059.

Royle NJ, Schuett W, Dall SRX. 2010. Behavioral consistency and the resolution of sexual conflict over parental investment. Behav. Ecol. 21:1125-1130. doi:10.1093/beheco/arq156. 
Rubenstein DR. 2007. Female extrapair mate choice in a cooperative breeder: trading sex for help and increasing offspring heterozygosity. Proc. R. Soc. B Biol. Sci. 274:1895-1903. doi:10.1098/rspb.2007.0424.

Ruuskanen S, Groothuis TGG, Baugh AT, Schaper S V., de Vries B, van Oers K. 2017. Maternal egg hormones in the mating context: The effect of pair personality. Funct. Ecol.:439-449. doi:10.1111/1365-2435.12987.

Ryder TB, Tori WP, Blake JG, Loiselle BA, Parker PG. 2010. Mate choice for genetic quality: A test of the heterozygosity and compatibility hypotheses in a lek-breeding bird. Behav. Ecol. 21:203-210. doi:10.1093/beheco/arp176.

Saaristo M, Craft JA, Lehtonen KK, Lindström K. 2009. Sand goby (Pomatoschistus minutus) males exposed to an endocrine disrupting chemical fail in nest and mate competition. Horm. Behav. 56:315-321. doi:10.1016/j.yhbeh.2009.06.010.

Saaristo M, Craft JA, Lehtonen KK, Lindström K. 2010. An endocrine disrupting chemical changes courtship and parental care in the sand goby. Aquat. Toxicol. 97:285-292. doi:10.1016/j.aquatox.2009.12.015.

Saladin V, Bonfils D, Binz T, Richner H. 2003. Isolation and characterization of 16 microsatellite loci in the Great Tit Parus major. Mol. Ecol. Notes 3:520-522. doi:10.1046/j.1471-8286.2003.00498.x.

Saladin V, Richner H. 2012. A set of 48 microsatellite loci for the great tit Parus major including 15 novel markers. Mol. Ecol. Resour. 12:185-189.

Sánchez-Tójar A, Nakagawa S, Sánchez-Fortún M, Martin DA, Ramani S, Girndt A, Bókony V, Kempenaers B, Liker A, Westneat DF, et al. 2018. Meta-analysis challenges a textbook example of status signalling: evidence for publication bias. bioRxiv:1-41. doi:10.1101/283150.

Sanderson JL, Wang J, Vitikainen EIK, Cant MA, Nichols HJ. 2015. Banded mongooses avoid inbreeding when mating with members of the same natal group. Mol. Ecol. 24:3738-3751. doi:10.1111/mec.13253.

Sappington TW, Taylor OR. 1990. Disruptive sexual selection in Colias eurytheme butterflies. Proc. Natl. Acad. Sci. USA 87:6132-6135. doi:10.1073/pnas.87.16.6132.

Scales J, Hyman J, Hughes M. 2013. Fortune favours the aggressive: Territory quality and behavioural syndromes in song sparrows, Melospiza melodia. Anim. Behav. 85:441-451. doi:10.1016/j.anbehav.2012.12.004.

Schuett W. 2008. Sexual selection and personality in zebra finches, Taeniopygia Guttata. $\mathrm{PhD}$ thesis, University of Exeter.

Schuett W, Dall SRX, Royle NJ. 2011. Pairs of zebra finches with similar "personalities" make better parents. Anim. Behav. 81:609-618. doi:10.1016/j.anbehav.2010.12.006.

Schuett W, Tregenza T, Dall SRX. 2010. Sexual selection and animal personality. Biol. Rev. Camb. Philos. Soc. 85:217-46. doi:10.1111/j.1469-185X.2009.00101.x.

Seddon N, Amos W, Mulder RA, Tobias JA. 2004. Male heterozygosity predicts territory size, song structure and reproductive success in a cooperatively breeding bird. Proc. R. Soc. B Biol. Sci. 271:1823-1829. doi:10.1098/rspb.2004.2805. 
Senar JC, Figuerola J, Pascual J. 2002. Brighter yellow blue tits make better parents. Proc. R. Soc. B Biol. Sci. 269:257-261. doi:10.1098/rspb.2001.1882.

Senar JC, Negro JJ, Quesada J, Ruiz I, Garrido J. 2008. Two pieces of information in a single trait? The yellow breast of the great tit (Parus major) reflects both pigment acquisition and body condition. :1195-1210.

Sepil I, Radersma R, Santure AW, De Cauwer I, Slate J, Sheldon BC. 2015. No evidence for MHC class I based disassortative mating in a wild population of great tits. J. Evol. Biol.:n/a--n/a. doi:10.1111/jeb.12600.

Sheldon BC. 2000. Differential allocation: tests, mechanisms and implications. Trends Ecol. Evol. 15:397-402. doi:10.1016/S0169-5347(00)01953-4.

Da Silva A, Gaillard JM, Yoccoz NG, Hewison AJM, Galan M, Coulson T, Allainé D, Vial L, Delorme D, Van Laere G, et al. 2009. Heterozygosity-fitness correlations revealed by neutral and candidate gene markers in roe deer from a long-term study. Evolution (N. Y). 63:403-417. doi:10.1111/j.1558-5646.2008.00542.x.

Sinn DL, Apiolaza LA, Moltschaniwskyj NA. 2006. Heritability and fitness-related consequences of squid personality traits. J. Evol. Biol. 19:1437-1447. doi:10.1111/j.14209101.2006.01136.x.

Slagsvold T, Dale S, Sætre GP. 1994. Dawn singing in the great tit (Parus major): mate attraction, mate guarding, or territorial defence? Behaviour 131:115-138.

Smith BR, Blumstein DT. 2007. Fitness consequences of personality: a meta-analysis. Behav. Ecol. 19:448-455. doi:10.1093/beheco/arm144.

Spoon TR, Millam JR, Owings DH. 2006. The importance of mate behavioural compatibility in parenting and reproductive success by cockatiels, Nymphicus hollandicus. Anim. Behav. 71:315-326. doi:10.1016/j.anbehav.2005.03.034.

Spottiswoode C, Møller AP. 2004. Genetic similarity and hatching success in birds. Proc. R. Soc. B Biol. Sci. 271:267-272. doi:10.1098/rspb.2003.2605.

Stoddard MC, Prum RO. 2008. Evolution of avian plumage color in a tetrahedral color space: a phylogenetic analysis of new world buntings. Am. Nat. 171:755-776. doi:10.1086/587526.

Stoffel MA, Esser M, Kardos M, Humble E, Nichols HJ, David P, Hoffman JI, Poisot T. 2016. inbreedR: an R package for the analysis of inbreeding based on genetic markers. Methods Ecol. Evol. 7:1331-1339. doi:10.1111/2041-210X.12588.

Sundin J, Berglund A, Rosenqvist G. 2010. Turbidity hampers mate choice in a pipefish. Ethology 116:713-721. doi:10.1111/j.1439-0310.2010.01787.x.

Szulkin M, Chapman JR, Patrick SC, Sheldon BC. 2012. Promiscuity, inbreeding and dispersal propensity in great tits. Anim. Behav. 84:1363-1370. doi:10.1016/j.anbehav.2012.08.030.

Szulkin M, Garant D, Mccleery RH, Sheldon BC. 2007. Inbreeding depression along a lifehistory continuum in the great tit. J. Evol. Biol. 20:1531-1543. doi:10.1111/j.14209101.2007.01325.x. 
Szulkin M, Sheldon BC. 2007. The environmental dependence of inbreeding depression in a wild bird population. PLoS One 2. doi:10.1371/journal.pone.0001027.

Szulkin M, Zelazowski P, Nicholson G, Sheldon BC. 2009. Inbreeding avoidance under different null models of random mating in the great tit. J. Anim. Ecol. 78:778-788. doi:10.1111/j.1365-2656.2009.01544.x.

Tinbergen JM, Daan S. 1990. Family planning in the great tit (Parus major): Optimal Clutch Size as Integration of Parent and Offspring Fitness. Behaviour 114:161-190.

Tomiuk J, Stauss M, Segelbacher G, Fietz J, Kömpf J, Bachmann L. 2007. Female genetic heterogeneity affects the reproduction of great tits (Parus major L., 1758) in low-quality woodlands. J. Zool. Syst. Evol. Res. 45:144-150. doi:10.1111/j.1439-0469.2006.00382.x.

Toomey MB, McGraw KJ. 2012. Mate choice for a male carotenoid-based ornament is linked to female dietary carotenoid intake and accumulation. BMC Evol. Biol. 12:3. doi:10.1186/1471-2148-12-3.

Tregenza T, Wedell N. 2000. Genetic compatibility, mate choice and patterns of parentage: Invited review. Mol. Ecol. 9:1013-1027. doi:10.1046/j.1365-294X.2000.00964.x.

Trivers RLL. 1972. Parental investment and sexual selection. In: Sexual Selection and the Descent of Man 1871-1971 (ed B Campbell). Aldine press. p. 136-179.

Tuomainen U, Candolin U. 2011. Behavioural responses to human-induced environmental change. Biol. Rev. 86:640-657. doi:10.1111/j.1469-185X.2010.00164.x.

Turner GF, Burrows MT. 1995. A model of sympatric speciation by sexual selection. Proc: Biol. Sci. 260:287-292.

Vahed K. 1998. The function of nuptial feeding in insects-review of empirical studies. Biol. Rev. 73:43-78.

Verbeek MEM, Boon A, Drent PJ. 1996. Exploration, Aggressive Behaviour and Dominance in Pair-Wise Confrontations of Juvenile Male Great Tits. Behaviour 133:945963.

Verbeek MEM, Drent PJ, Wiepkema PR. 1994. Consistent individual differences in early exploratory behaviour of male great tits. Anim. Behav. 48:1113-1121. doi:10.1006/anbe.1994.1344.

Voegeli B, Saladin V, Wegmann M, Richner H. 2012. Parasites as mediators of heterozygosity-fitness correlations in the Great Tit (Parus major). J. Evol. Biol. 25:584-590. doi:10.1111/j.1420-9101.2011.02445.x.

Wagner WEJ. 1998. Measuring female mating preferences. Anim. Behav. 55:1029-42. doi:10.1006/anbe.1997.0635.

Wang D, Forstmeier W, Kempenaers B. 2017. No mutual mate choice for quality in zebra finches: Time to question a widely-held assumption. Evolution (N. Y).:2661-2676. doi:10.1111/evo.13341.

Wang J. 2002. An estimator for pairwise relatedness using molecular markers. Genetics 160:1203-1215. 
Wang J. 2011. Coancestry: A program for simulating, estimating and analysing relatedness and inbreeding coefficients. Mol. Ecol. Resour. 11:141-145. doi:10.1111/j.17550998.2010.02885.x.

Wang MT, Hsu YC, Yao C Te, Li SH. 2005. Isolation and characterization of 12 tetranucleotide repeat microsatellite loci from the green-backed tit (Parus monticolus). Mol. Ecol. Notes 5:439-442. doi:10.1111/j.1471-8286.2005.00955.x.

Weddle CB, Hunt J, Sakaluk SK. 2013. Self-referent phenotype matching and its role in female mate choice in arthropods. Curr. Zool. 59:239-248.

Werner EE, Hall DJ. 1974. Optimal foraging and the size selection of prey by the Bluegill Sunfish (Lepomis Macrochirus). Ecology 55:1042-1052. doi:10.2307/1940354.

Whittingham LA, Dunn PO. 2005. Effects of extra-pair and within-pair reproductive success on the opportunity for selection in birds. Behav. Ecol. 16:138-144. doi:10.1093/beheco/arh140.

Widemo F, Sæther SA. 1999. Beauty is in the eye of the beholder: causes and consequences of variation in mating preferences. Trends Ecol. Evol. 14:26-31. doi:10.1016/S01695347(98)01531-6.

Wingfield JC, Hegner RE, Dufty, AM, Ball GF. 1990. The "Challenge Hypothesis": Theoretical implications for patterns of testosterone secretion, mating systems, and breeding strategies. Am. Nat. 136:829-846. doi:10.1086/285134.

Witte K. 2006. Time spent with a male is a good indicator of mate preference in female zebra finches. Ethol. Ecol. Evol. 18:195-204. doi:10.1080/08927014.2006.9522707.

Zahavi A. 1975. Mate selection-a selection for a handicap. J. Theor. Biol. 53:205-214.

Zandberg L, Gort G, van Oers K, Hinde CA. 2017. Direct fitness benefits explain mate preference, but not choice, for similarity in heterozygosity levels. Ecol. Lett. 20:1306-1314. doi:10.1111/ele.12827.

Zeh JA, Zeh DW. 1996. The Evolution of Polyandry I: Intragenomic conflict and genetic incompatibility. Proc. R. Soc. B Biol. Sci. 263:1711-1717. doi:10.1098/rspb.1996.0250.

Zuk M, Johnsen TS, Maclarty T. 1995. Endocrine-immune interactions, ornaments and mate choice in Red Jungle Fowl. Proc. R. Soc. B Biol. Sci. 260:205-210.

Zuk M, Thornhill R, Ligon JD, Johnson K, Austad S. 1990. The role of male ornaments and courtship behavior in female mate choice of Red Jungle Fowl. Am. Nat. 136:459-473. 



\section{Summary}

By choosing the right partner individuals can gain reproductive benefits and can increase their reproductive success. These benefits can be direct, when the offspring's quality or quantity is increased by the behaviour or investment of the mate, or indirect, when the quality of the offspring is increased by the genetic contribution of the mate. While previously it was often thought that reproductive benefits were primarily caused by the higher quality of these preferred partners, more and more studies suggest that also partner compatibility may increase reproductive success.

Mate compatibility may be especially important when individuals differ in their preferences for a mate. Although uniform preferences are often assumed, an increasing number of studies indicate that individuals have a preference for different trait values (Forstmeier and Birkhead 2004; Lehtonen and Lindström 2008; Holveck and Riebel 2010; Ihle et al. 2015). Moreover, these differences in preference can depend on the chooser's own trait values (Mays and Hill 2004; Holveck and Riebel 2007). These studies show that individuals may prefer a mate that is the best fit for them, rather than the universally 'best individual'.

A number of studies on captive populations have found that the fitness benefits from mating with a certain individual differ between individuals, suggesting individual differences in preference and both direct and indirect benefits of mating with these compatible mates. However, it is still unclear how compatibility and perceived mate attractiveness affect reproductive success in a wild population. Additionally, to understand fitness consequences and the evolution of mate preferences, preference and choice should be studied as two distinct processes. To our knowledge, no study thus far has combined all three and tested what the fitness benefits of mate preferences are under mate choice constraints in a wild population. Therefore, the overall aim of this thesis was to study the role of individual differences in mate preferences and its effects on reproductive success, by comparing measured mate preference with the resulting choice and relating the pair compatibility with different aspects of reproductive success.

When measuring mate preferences results may be affected by how individuals evaluate and compare the available options they are presented with. Preferences are often assumed to be absolute, assigning a fixed, absolute value to a cue or potential partner they encounter. However, in reality, individuals may compare the available options, in which case the social context plays an essential role in the preference for each potential partner. In chapter 2 I used a 6-choice mate choice setup to test great tits for their preferences in a mate. Using such a test design allowed individuals to comparatively evaluate potential 
partners. Additionally, presenting individuals with 6 choice options allowed for the measurement of non-linear preferences. I reveal that great tits use a combination of both absolute and comparative evaluation and that the social context affected the measured responses to stimulus groups. This suggests that great tits can flexibly use both evaluation methods, depending on the situation they encounter potential mates in. With such knowledge of how a study species encounters and evaluates potential mates, it is possible to choose the most appropriate experimental design and analysis to obtain reliable measurements of mate preferences. The ability to more accurately quantify preference is expected to increase our understanding of mate preferences, mate choice, and ultimately sexual selection.

By measuring preferences for traits in a mate I found that individuals differed in their preferences for a mate. In chapter 4 I have found that there were no population-wide mate preferences for the different bird traits that we measured (heterozygosity, intensity yellow breast plumage, black breast stripe size and body condition) and that individual preference slopes for (at least three of) these traits differed between individuals. Thus, rather than preferring the same universally attractive individuals, individuals may prefer a mate that is compatible to themselves. Moreover, individuals may differ in which traits they find important in a mate (chapter 4).

Compatibility between pair mates can be dependent on the combination of the pairs' trait values. In chapter 3 I have shown that preferences for certain traits were dependent on the choosers' own traits, suggesting preference for compatibility for these traits. By testing individual repeatedly for their mate preferences for genetic characteristics I found that both sexes preferred mates that had heterozygosity levels similar to themselves, and not those with which they would optimise offspring heterozygosity. Moreover, I found that these preferences for similarity in heterozygosity were in reflected in direct benefits for reproductive success. In the subsequent field experiment where we cross fostered offspring, foster parents with more similar heterozygosity levels had higher reproductive success, despite the absence of assortative mating patterns. This suggests that direct fitness benefits underlie mate preferences for genetic characteristics and that the potential for selection for preferences persists despite constraints on mate choice.

Another trait that is thought to play a large role in pair compatibility is behavioural compatibility. Especially for species with biparental care behavioural traits, such as personality traits and behavioural compatibility may be important for reproductive success. In chapter 5 I studied the role of personality and behavioural compatibility in the provisioning behaviour in particular, by recording parental provisioning behaviour of great tit pairs with known exploratory behaviour. By experimentally cross-fostering 
offspring I was able to distinguish between the pre-hatching and rearing effects of parental personality on offspring weight and fledging probability. Here I found that the combination of personalities in a pair did not affect their provisioning behaviour. Instead, I found that although all birds provision on a trade-off between visit rate and prey volume, fast birds brought in smaller prey for a given visit rate resulting in a lower overall amount of prey delivered to their offspring. These differences in provisioning strategy led to faster explorers bringing a lower total prey volume to their offspring. Thus, personality may certainly play a role in direct benefits that individuals gain from finding a mate, however, this benefit was not dependent on the choosers' own traits. Despite the personality dependent differences in provisioning behaviour, there was no effect of pair compatibility on provisioning performance. However, it is possible that these effects of personality are context dependent, or only become apparent when pairs encounter challenges in their breeding attempt. To gain a better insight into how selection pressures may favour certain combinations of personalities these personality effects should be measured over more different contexts (over different years, different contexts or using experimental manipulations). Additionally, a context dependence of behavioural compatibility suggests a fluctuating selection on personality traits and pair compatibility, and with it a potential for fluctuating selection on preferences for mate personality.

Individuals have been shown to have increased reproductive success when they are able to mate with individuals that they perceive as attractive. Since here individuals have been found to differ in their preferences, and some traits can be more or less important for different individuals depending on their own traits (chapter 3 and 4), each individual can differ in how attractive they perceive the same potential mate. This has been shown in captivity where it has been found that the same courter may give higher benefits to some choosers than to others. Here I investigated if the attractiveness of an individual, as perceived by its mate, increased the reproductive success of the pair (chapter 4). On the basis of preference tests I calculated preference functions for each individual and in the subsequent breeding season estimated a measure of how attractive each individual perceived the mate they were paired with. Here I found that while individuals differed in their preference for a mate, they were not more likely to obtain a mate that they perceived as attractive than under random mating. Additionally, I found no effects of perceived mate attractiveness on female reproductive investment, offspring weight or fledging probability. Thus, although with a limited sample size, we found no effect of our measure individual mate attractiveness on reproductive success, this method of estimating preferences and individual mate attractiveness gives the potential for further studies studying pair compatibility and reproductive success. A better understanding of 
preferences for compatibility rather than quality and the benefits related to this may shed light on the adaptive value of individual differences in preference for mates.

By measuring both preference and choice I found that, quite often, preferences are not reflected in the mating patterns that we find in the population (chapter 3 and 4 ). However, I did find that these preferences were reflected in reproductive benefits (chapter 3). Thus, while not always being able to, those individuals that did find a mate according to their preferences benefitted from compatibility with their mate. Mate choice is therefore the result of choosing a partner according to ones' preferences whilst under physical and environmental constraints. This emphasizes the importance of measuring both preference and choice to fully understand how sexual selection acts on preferences.

To conclude, in this thesis I investigated the role of compatibility on mate preferences, mate choice and reproductive success. Additionally, I revealed how individuals evaluate potential partners and how perceived mate attractiveness may affect reproductive success. Here I have found that mate preferences for a compatible, rather than universally attractive, partner may have evolved to gain direct or indirect benefits from pairing. Additionally, while there may be constraints on the choice of a mate, finding a compatible mate can increase reproductive success. These results also illustrate the importance of considering mate preferences and mate choice two distinct processes, both of which should be studied to better understand how sexual selection acts on preferences. 




\section{Samenvatting}

Het kiezen van een geschikte partner is van groot belang. Immers, door de juiste partner te kiezen kunnen dieren voordelen behalen voor hun voortplanting en daarmee hun voortplantingssucces vergroten. Deze voordelen kunnen direct zijn, wanneer de kwaliteit of de kwantiteit van de jongen hoger is door het gedrag of de investeringen van de partner, of indirect, wanneer de kwaliteit van de jongen hoger is dankzij de genen van de gekozen partner. Eerder dacht men dat deze reproductieve voordelen van partnerkeuze voornamelijk veroorzaakt werden door de hogere kwaliteit van deze partners, maar tegenwoordig zijn er steeds meer studies die suggereren dat de compatibiliteit tussen partners ook van belang is om een hoger voortplantingssucces te behalen.

Deze compatibiliteit tussen partners kan met name een rol spelen in diersoorten waar individuen verschillen in hun voorkeur voor een partner. Hoewel het vaak aangenomen wordt dat partnervoorkeuren uniform zijn, zijn er toch steeds meer studies die aangeven dat individuen verschillen in hun voorkeur voor bepaalde uiterlijke of innerlijke eigenschappen in een partner. Bovendien kunnen deze voorkeuren weer afhangen van de kiezers' eigen eigenschappen. Deze studies geven aan dat dieren niet een voorkeur hebben voor het universele 'beste' individu, maar voor de partner die het beste bij hen past.

Verschillende studies aan dieren in gevangenschap hebben aangetoond dat individuen verschillen in hun voorkeur voor een partner en dat het voorplantingssucces van het paren met de ene partner hoger is, dan wanneer ze gekoppeld worden aan andere partner. De ene combinatie bleek succesvoller dan de andere, ongeacht de kwaliteit van elk van de partners. Dit suggereert niet alleen dat er individuele verschillen zijn in voorkeur, maar ook dat er zowel directe als indirecte voordelen te behalen zijn door met een compatibel individu te paren. Wat alleen nog niet duidelijk is, is hoe compatibiliteit met de partner en de aantrekkelijkheid van de partner reproductief succes beïnvloedt in een natuurlijke populatie. Zeker in wilde populaties kan dit effect van compatibiliteit relevant zijn omdat het in de natuur niet altijd mogelijk is om een aantrekkelijke of compatibele partner te vinden. Bovendien, om de gevolgen voor de fitness en de evolutie van partner voorkeuren te begrijpen, moeten zowel de voorkeuren als de uiteindelijke keuze voor een partner gemeten worden omdat de combinatie van deze twee processen samen bepalend zijn voor het voortplantingssucces en daarmee de seksuele selectie. Voor zover wij weten is er tot nu toe geen studie geweest die, in een wilde populatie deze drie aspecten, voorkeur, keuze en reproductief succes gecombineerd hebben en vervolgens getest hebben wat de fitness voordelen van het hebben van partnervoorkeuren is onder beperkte partnerkeuze. Daarom is het doel van deze thesis om de rol van individuele verschillen in 
partnervoorkeur en de effecten daarvan op reproductief succes te bestuderen. Dit doen we door de partnervoorkeuren te meten en te vergelijken met de partnerkeuze die uiteindelijk gemaakt is om de paar-compatibiliteit te relateren aan de verschillende aspecten van reproductief succes.

Wanneer men partnervoorkeuren meet kunnen de resultaten beïnvloed worden door de manier waarop individuen de beschikbare opties evalueren en vergelijken. Vaak wordt gedacht dat de voorkeuren 'absoluut' zijn, waarbij een vaste absolute waarde van aantrekkelijkheid wordt verbonden aan een bepaalde eigenschap of een potentiële partner. In realiteit echter, kunnen individuen ook de beschikbare opties vergelijken. In dit geval spelen de beschikbare opties, of de sociale context, een essentiële rol in de voorkeur voor elke potentiele partner. In hoofdstuk 2 heb ik de partnervoorkeuren van wilde koolmezen getest door ze met een 6-keuze testopstelling te testen. Door middel van dit meerkeuze testontwerp kunnen de kiezers de potentieel beschikbare partners vergelijken. Bovendien, door de kiezers te presenteren met 6 opties is het mogelijk om ook non-lineaire voorkeuren te meten. Hier laat ik zien dat koolmezen de potentieel beschikbare partners evalueren door een combinatie van zowel absolute als vergelijkende evaluatie, en dat de sociale context de gemeten voorkeuren beïnvloedt. Dit suggereert dat koolmezen, afhankelijk van de situatie waarin zij mogelijke partners tegenkomen, flexibel zijn in het gebruiken van beide evaluatiemethoden. Met dergelijke kennis over hoe een studiesoort potentiele partners tegenkomt en evalueert, is het mogelijk om de meest passende experimentele aanpak en analyses te kiezen om partnervoorkeuren zo betrouwbaar mogelijk te kunnen meten. Door deze voorkeuren accurater te kwantificeren kunnen we onze kennis over partnervoorkeuren, partnerkeuze, en uiteindelijk seksuele selectie vergroten.

Door het meten van de voorkeuren voor verschillende eigenschappen heb ik gevonden dat individuen verschilden in hun voorkeuren voor een partner. In hoofdstuk 4 heb ik bijvoorbeeld gevonden dat er, voor de eigenschappen die wij gemeten hebben (heterozygositeit, intensiteit van de gele borstveren, formaat van de zwarte borststreep, en de lichaamsconditie), geen sprake was van uniforme partnervoorkeuren in de deze populatie en dat individuele voorkeursfuncties voor (tenminste drie van deze eigenschappen) verschilden tussen individuen. Dus, in plaats van dat elk individu dezelfde voorkeur voor dezelfde universeel aantrekkelijke individuen heeft, heeft elk individu zijn eigen voorkeur voor een compatibele partner. Daarnaast kunnen individuen ook verschillen in welke eigenschappen ze belangrijk vinden in een partner (hoofdstuk $4)$. 
Compatibiliteit binnen een paar kan afhangen van de combinatie van de eigenschappen van deze beide partners. In hoofdstuk 3 laat ik zien dat voorkeuren voor bepaalde eigenschappen afhankelijk waren van de kiezers' eigen eigenschappen. Dit suggereert dat ze een voorkeur hebben voor compatibiliteit voor deze eigenschappen. Door individuen herhaaldelijk voor hun voorkeuren voor genetische eigenschappen te testen heb ik gevonden dat zowel mannelijke als vrouwelijke koolmezen een voorkeur hebben voor een partner die net zo heterozygoot is als zichzelf, en niet, zoals we verwacht hadden, een partner waarmee ze heterozygote nakomelingen zouden krijgen. Daarnaast heb ik gevonden dat deze voorkeuren voor gelijke heterozygositeit in een partner weerspiegeld werden in het broedsucces die ze kregen van het vinden van een dergelijke, compatibele partner. In een opvolgend veldexperiment heb ik jongen uitgewisseld tussen nesten om onderscheid te kunnen maken tussen de effecten van de opvoeding van de pleegouders en de effecten van de biologische ouders (bijvoorbeeld genen, hormonen en andere investeringen in het ei van de moeder) op de groei of uitvliegkansen van de jongen. Met dit experiment heb ik gevonden dat wanneer de pleegouders gelijke niveaus van heterozygositeit hadden, ze beter waren in het opvoeden van de jongen. Maar ondanks de directe voordelen voor de voortplanting, was er geen sprake van een assortatief keuze patroon, waarin heterozygote dieren vaker paarden met heterozygote dieren en vice versa voor de homozygote dieren. Dit suggereert dat, ondanks beperkingen in de keuze van een compatibele partner, de partnervoorkeuren voor genetische eigenschappen toch tot stand kunnen komen, en kunnen blijven bestaan, dankzij de directe voordelen voor reproductie.

Een andere eigenschap die een rol kan spelen in paar-compatibiliteit is compatibiliteit in gedrag. Vooral voor soorten waarbij beide ouders een rol spelen in het opvoeden van de jongen kan dit van belang zijn voor hun reproductieve succes. In hoofdstuk 5 heb ik de rol van comptabiliteit op het gebied van gedrag, in het bijzonder van persoonlijkheid, in het voeren van de jongen bestudeerd. Dit heb ik gedaan door het voergedrag van koolmees- paren van wie het exploratiegedrag (gebruikt als meting van persoonlijkheid) bekend was, te observeren. Door experimenteel de jongen tussen nesten tussen nesten uit te wisselen heb ik gevonden dat de combinatie van persoonlijkheden in een paar geen effect had op hun voergedrag. In plaats daarvan heb ik gevonden dat, ondanks dat alle vogels een 'trade-off' hebben wanneer ze hun jongen voeren, een afweging tussen hoe vaak ze bezoeken en hoe groot de prooi is die ze brengen, vogels met snel exploratiegedrag, vaak voor een gegeven aantal bezoeken gemiddeld kleinere prooien binnenbrengen. Deze verschillen in voerstrategieën leiden ertoe dat deze 'snelle' dieren een lagere totale hoeveelheid aan prooien aan hun jongen voeren. Persoonlijkheid kan dus zeker een rol spelen in de keuze voor een partner en de voordelen (of nadelen) voor voortplanting die daarmee gemoeid zijn. Toch, is dit voordeel niet afhankelijk van de 
persoonlijkheid van de kiezer zelf: ondanks de persoonlijkheidsafhankelijke verschillen in voergedrag, was er geen effect van paar-gedragscompatibiliteit op de voerprestaties. Het is echter ook mogelijk dat deze effecten van persoonlijkheid op broedsucces afhankelijk zijn van de omgeving, of dat deze effecten alleen tot uitdrukking komen wanneer paren moeilijke omstandigheden tegenkomen tijdens het opvoeden van hun jongen. Om hier een beter begrip van te krijgen zouden deze persoonlijkheidseffecten in verschillende situaties gemeten moeten worden (verschillende jaren, omstandigheden of omgevingen of onder verschillende experimentele manipulaties). Bovendien, wanneer gedragscompatibiliteit afhankelijk is van de context, dan suggereert dit dat er ook sprake kan zijn van een wisselende selectie op voorkeuren voor persoonlijkheid in een partner.

Het is aangetoond dat individuen die de kans krijgen om zich voort te planten met een partner die ze die ze aantrekkelijk vonden een hoger reproductief succes hebben. Omdat ik gevonden heb dat individuen verschillen in hun voorkeur voor bepaalde eigenschappen, en individuen ook verschillen in hoe belangrijk ze de verschillende eigenschappen in een partner vinden afhankelijk van hun eigen eigenschappen (hoofstukken 3 en 4), kan elk individu ook verschillen in hoe aantrekkelijk ze bepaalde potentiele partners vinden. Dit is voorheen al aangetoond waar eenzelfde individu in gevangenschap hogere reproductieve voordelen kan geven aan de ene partner dan aan de andere partner. Ik heb onderzocht of de aantrekkelijkheid van een individu, vanuit het oogpunt van zijn partner, het reproductieve succes van een paar kan verhogen. Op basis van voorkeurstesten heb ik de voorkeursfuncties van elk individu berekend. In het opvolgende broedseizoen heb ik op basis van deze functies een schatting gemaakt van hoe aantrekkelijk ze de partner die ze uiteindelijk gekozen hebben zouden vinden. Hier heb ik gevonden dat, ondanks dat individuen verschilden in hun voorkeur voor een partner, de kans dat zij een partner kregen die zij aantrekkelijk vonden niet hoger was dan de kans dat ze een onaantrekkelijke partner vonden. Bovendien heb ik ook geen effecten gevonden van deze partneraantrekkelijkheid (zoals gezien door hun eigen partner) op de investering in voortplanting door de vrouwtjes, op de groei van de jongen of hun uitvliegkansen. Dus, ondanks dat we, met een relatief kleine steekproefomvang, geen effect gevonden van onze schatting van partneraantrekkelijkheid op broedsucces, geeft deze methode van het schatten van voorkeuren en een mate van partneraantrekkelijkheid wel het potentieel voor verdere studies die het verband tussen paarcompatibiliteit en voortplantingssucces bestuderen. Een beter begrip van voorkeuren voor compatibiliteit in plaats van voor kwaliteit in een partner en de voordelen die hieraan gerelateerd zijn kunnen zou een verklaring kunnen geven voor de adaptieve waarde van individuele verschillen in voorkeuren voor een partner. 
Dankzij het meten van zowel de voorkeur als de uiteindelijke keuze voor een partner heb ik gevonden dat, in veel gevallen, de voorkeuren niet terugkomen in de uiteindelijke keuze en de resulterende keuzepatronen in de populatie (hoofdstukken 3 en 4). Ik heb echter wel gevonden dat deze voorkeuren terugkomen in de voordelen voor het broedsucces die individuen krijgen van hun keuze (hoofdstuk 3). Dus, ondanks dat het niet altijd mogelijk is om een partner te vinden die aan hun voorkeur voldoet, hebben de individuen die wel een dergelijke partner hebben kunnen vinden een voordeel van het hebben van een dergelijke compatibele partner. Partnerkeuze is dus het resultaat van de keuze die individuen moeten maken op basis van hun voorkeuren, terwijl ze beperkt worden door bijvoorbeeld hun eigen fysieke gesteldheid of uiteenlopende omgevingsfactoren. Dit benadrukt het belang van het meten van zowel voorkeur als keuze om echt een beter begrip te krijgen van hoe partnervoorkeuren ontstaan en blijven bestaan onder seksuele selectie.

In conclusie, in deze thesis heb ik de rol van compatibiliteit in partnervoorkeuren, partnerkeuze en reproductief succes onderzocht. Verder heb ik laten zien hoe individuen potentiële partners evalueren en hoe aantrekkelijkheid, zoals gezien door hun partner, reproductief succes kan beïnvloeden. Ik heb gevonden dat partnervoorkeuren voor compatibele partners, in plaats van universeel aantrekkelijke partners, ontstaan kunnen zijn door de directe of indirecte voordelen die individuen krijgen wanneer ze met een compatibele partner paren. 



\section{Curriculum Vitae}

Elisabeth Christina Ada Zandberg, also better known as Lies, was born on 23th of March 1987 in Tilburg, the Netherlands. She grew up in Deventer and moved to Wageningen in 2005 to start her Bachelor in Biology at Wageningen University. In 2010 she obtained this BSc degree with a specialisation in Animal Biology.

Following her BSc, Lies continued at the same university with her MSc in Biology, again specialising in Animal Biology. For her minor thesis she performed

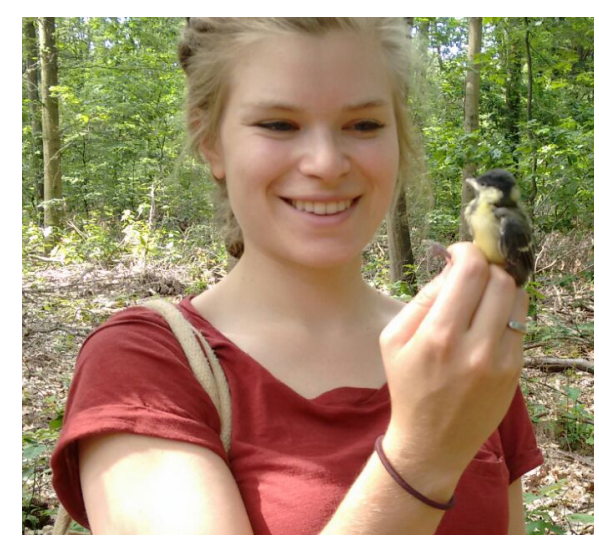
a social network analysis on a herd of horse and studied the effects of removal of different individuals on the network. Subsequently for her major thesis she joined the group of Alex Thornton at the University of Cambridge to study the development of vocal recognition in jackdaw nestlings (Corvus monedula). Now being infected with the 'bird-virus' she chose to do another research project on birds, this time with Kees van Oers at the Netherlands Institute of Ecology (NIOO-KNAW), where she studied the influence of personality on foraging and problem solving in a social context in great tits (Parus major). Lies obtained her MSc degree in 2013 with the distinction cum laude.

After her obtaining her MSc Lies started her PhD project studying sexual selection and mate choice in great tits in the Behavioural Ecology group at Wageningen University and the Department of Animal Ecology of the Netherlands Institute of Ecology (NIOOKNAW). Supervised by Dr. Camilla Hinde and Dr. Kees van Oers she studied the role of compatibility in mate preferences in great tits. For this work she was awarded the Storm van der Chijs stipendium for most talented female PhD student at Wageningen University in 2017.

Lies has now started as a postdoctoral research assistent at Queen Mary University London working on the perception and cultural evolution of song in birds with Robert Lachlan. 



\section{Acknowledgements}

First, I would like to thank you, Camilla. Thank you for taking a chance on me with this project. Even though I might not have been so much of an ecologist when I started out on this project, I would say I definitely am now! It's been absolutely wonderful to have you as my supervisor. You inspire me to always go for the best possible option, even though this may not always be the easiest route to take. I am extremely grateful for all your advice and support.

Kees, thank you so much for always trusting in me and my abilities, and giving me the confidence to pursue a career in science. In a way it all started with doing my MSc intership with you at the NIOO-KNAW, when you said that I could and should definitely find a $\mathrm{PhD}$ position if I wanted to. And I am very happy that I took your advice. Thank you for all the discussions, about scientific and non-scientific topics, and for always making time for a chat.

I couldn't have been happier with the help of my two paranymphs during the last stretch of my PhD. Lydia, your office has been my favorite stop on my way to the coffee machine. Thank you so much for our chats about everything: about happiness and frustrations, about fluffy animals and basically about life. Especially in times of stress it's great to have someone to vent to about it and then someone to laugh about it with. Sabine, having a coffee with you invariably leads to good conversations. I love your openmindedness and how you can be interested in and enthusiastic about pretty much anything. And I admire the way that you can put your work into perspective and are able to prioritise the important things in life.

I would also like to thank all my colleagues at the Behavioural Ecology Group. It has been really great to be part of this group and see it grow and change over the years. We've had a lot of fun with our outings such as pub quizzes, escape rooms, watching rutting deer, and of course the legendary visits to the Efteling. Lysanne, you were my first PhD buddy at BHE. Thank you for introducing me to the world of science communication with WEES and Beleef de Lente. I think we made a great Buurten bij de Buren team! I also really liked our discussions about the scientific world in general and about animal behaviour and welfare. Since the coffee at Zodiac is not the best in the world, I was very happy to have found some colleagues who, just like me, are pretty much always up for a proper cup of coffee, even if we have to take a walk to get it. Nina, it's been great to have another colleague working on great tits. Your determination and thoroughness are absolutely admirable. Nienke, your friendliness and positivity can be felt every time someone walks into your office. Thank you for always being helpful and cheerful in doing so. Jerine, 
Anne, Elske and Malou it's been great having you around in our group. I really enjoyed our lunch time discussions! James and Shana thank you both for your statistical advice and for being wonderful lunch companions. And even though you haven't been in our group for very long yet, Filipe, seeing your grin peering over my computerscreen always put me in a good mood (although all those chocolates you smuggled into our room probably also helped with that!). It's been absolutely great fun having all of you around. Bas, Bonne, Jutta and Joanne, I really enjoyed being in such a diverse group with a mix of applied and fundamental science. Looking at the same thing from different perspectives can enrich and improve our understanding of these things. And last, but certainly not least, Marc, thank you for being my promotor and for always taking the time to give me advice when I needed it.

It's been very inspiring to be able to work at two great scientific institutions at the same time: Wageningen University and the Netherlands Institute for Ecology. I would like to thank all the colleagues at this 'second home', the Department of Animal Ecology at the NIOO-KNAW for making my time there absolutely great. I have learned so much from all of you. Piet thank you for being my guide into the world of great tits. Your experience and advice have been so helpful in learning how to work with these birds. But apart from the fact that you are undeniably the biggest expert on great tits I have ever met, I also appreciate it enormously that you always have time to sit down to talk, about birds, about science, but also about all the other things in life. Christa, thank you so much for helping me with all the genetic analyses and of course for all our chats and all those great book suggestions (I still haven't been able to finish all of them!). And ofcourse a very big thank you to my PhD colleagues Lucia, Barbara, Maaike, Thomas, Davide, Jip, Irene, Rascha and Els. It was you guys who always made working in the $\mathrm{PhD}$ room a lot of fun! Peter and Lysanne, thank you for including me in 'Team Koolmees' for Beleef de Lente. Your enthusiasm about science communication is absolutely contagious! Marylou, Franca, Ruben, Anouk and Coretta thank you so much for helping me out with taking care of the birds. I know bringing in so many birds over the winter months took a lot of work, and a lot of flexibility to work around the experiments.

Many, many people have helped me out in the field, and without their help this project would never have been possible. And for everyone that joined on one of the many roostchecks to catch birds on cold winter evenings, I absolutely admire your tenacity in finding even those last hidden nest boxes in a dark forest! Moreover, most of the people who have helped me out in the field and/or with data analyses were students. Thank you: Maaike, Maxime, Lisanne, Kristin, Neeltje, Tjomme, Orestis, Kim, Moniek, Anne, Daniel, Tim, Lieske, Yfke, Pim, Annemiek and Coen. And many thanks to my field assistants Peter and Antoine who worked tirelessly to help me get all the work done by 
the end of the day, even at the busiest of field seasons. Without the help of so many great people I would never have been able to do this work. Thank you all!

Dear Mariska and Mirelle, I think our run training sessions during the last year of my PhD have played a large role in keeping me sane. Just clear your head and go. We should definitely get together in London to do this again! Judith, thank you so much for all those great little dinner parties we had, and ofcourse for making the fantastic cover of my thesis! Van jongs af aan was ik al geïnteresseerd in dieren. Het was daarom fantastisch om op te kunnen groeien omringd door een wilde verzameling dieren: vissen, hamsters, muizen, zebravinken, cavia's, ratten en een hond. Ik mag me dan ook gelukkig prijzen met een thuis dat me altijd gestimuleerd heeft om die passie voor dieren te volgen en er uiteindelijk ook mijn beroep van te maken! Mam, dankjewel voor je onvoorwaardelijke steun en liefde. Jouw liefde voor boeken, lezen en nieuwe dingen leren heb je zeker over weten te dragen op mij. Pap, mijn koppigheid en doorzettingsvermogen moet ik haast wel van jou hebben. En zoals jij met groot observatievermogen het gedrag van mensen vastlegt op je foto's, zo bestudeer ik het gedrag van dieren. Dankjewel dat je me altijd gestimuleerd hebt om er volledig voor te gaan. Fem, ook al denken we niet altijd hetzelfde over dingen, toch heb ik jouw advies en mening altijd ontzettend waardevol gevonden. Je bent mijn grote zus en ik zal waarschijnlijk altijd je 'vervelende kleine zusje' zijn, ik bewonder je doorzettingsvermogen en de manier waarop je volledig achter je standpunten staat enorm. Koen, mijn kleine broertje dat ondertussen best wel groot geworden is. Van kleine computernerd naar zeer gewaardeerde techneut. Ik vind het prachtig hoe je de dingen op jouw manier doet, op jouw tempo en hoe je je niet van de wijs laat brengen.

En als laatste lieve Lieven, het hele proces van mijn $\mathrm{PhD}$ ben je erbij geweest, van sollicitatie tot aan mijn defence. Je hebt mij altijd gestimuleerd om het beste uit mezelf te halen en nooit op te geven, en tegelijkertijd heb je me geholpen om dingen te relativeren wanneer dat nodig was. Ik ben ontzettend dankbaar voor je onuitputtelijke geduld op de momenten dat ik er even doorheen zat en voor al je steun, zowel praktisch als moreel. En nu samen op naar het volgende avontuur in Londen! 



\section{WIAS Training and Education Statement}

Completed in fulfilment of the requirements for the Education Certificate of the Wageningen Institute of Animal Sciences

The Basic Package

3 ECTS

WIAS Introduction Course

2014

Course on philosophy of science and/or ethics

2016

Scientific Exposure

19 ECTS

International conferences

Annual meeting of the Netherlands society for Behavioural Biology, Soesterberg,

International Society for Behavioral Ecology meeting (ISBE), New York, USA

2014

Association for the Study of Animal Behaviour (ASAB) winter meeting, London,

2014

UK

Annual meeting of the Netherlands society for Behavioural Biology (NVG),

2016

Soesterberg,

International Society for Behavioral Ecology meeting (ISBE), Exeter, UK

2016

Behaviour 2017, joint meeting International Ethological Conference (IEC) and

Association for the Study of Animal Behaviour (ASAB), Estoril, Portugal

Association for the Study of Animal Behaviour (ASAB) winter meeting, London,

UK

\section{Seminars and workshops}

PhD Workshop Netherlands society for Behavioural Biology, Soesterberg, NL

\section{Presentations}

$\underline{\text { Scientific presentations }}$

Oral presentation "How is phenotypic variation maintained by sexual selection?" at

Annual meeting of the Netherlands society for Behavioural Biology (NVG) PhD workshop, Soesterberg

Poster "Mate choice a matter of taste?" International Society for Behavioral

Ecology meeting (ISBE), New York, USA

Oral presentation "Mate preference a matter of taste? How is variation maintained by sexual selection?" NIOO/KNAW seminar

Oral presentation "Mate choice a matter of taste? Individual variation in mate

Oral presentation "Mate preference a matter of taste? Individuals differ in mate preferences for genetic traits." At International Society for Behavioral Ecology meeting (ISBE), Exeter, UK

Oral presentation "Preferences for genetic traits and the effects on reproductive success." Annual meeting of the Netherlands society for Behavioural Biology (NVG)

Oral presentation "Mate preferences for fitness benefits. Preference not choice for similarity in heterozygosity levels." Behaviour 2017, joint meeting International 
Ethological Conference (IEC) and Association for the Study of Animal Behaviour (ASAB), Estoril, Portugal

Poster "Mate preference for compatible partners. Preference not choice for similarity in heterozygosity levels." Association for the Study of Animal Behaviour (ASAB) winter meeting, London, UK

Presentations for 'layman'

Oral presentation "Partnervoorkeur bij koolmezen: een kwestie van smaak" for Biesbosch fieldguides at Biesboschcentrum

Oral presentation "Partnervoorkeur in koolmezen" for KNNV vereniging voor

Veldbiologie Wageningen

\section{In-Depth Studies}

Disciplinary and interdisciplinary courses

Life History Theory (RUG)

Workshop Personality 2015

Workshop Parental Provisioning

Advanced statistics courses

Meta analysis

PhD students' discussion groups

Department of Animal Ecology NIOO-KNAW, weekly journal club

2013-2016

Behavioural Ecology Group, Science meetings (+organisation of meetings)

2015-2017

\section{Professional Skills Support Courses}

Brain training

PhD Competence assessment or Job assessment

\section{Research Skills Training}

Reviewing journal articles: Animal Behaviour (3), Behavioral Processes (1)

\section{Didactic Skills Training}

\section{Supervising practicals and excursions}

Behavioural Ecology (BHE-30306)

Animal behaviour (BHE-20303)

\section{Supervising theses}

3 MSc (2 major, 1 minor)

\section{Scientific outreach}


"Beleef de Lente" expert blogs: writing a weekly blog during the great tit 2014-2018 breeding season on the website of the Dutch Society for the Protection of Birds (VBN; visited by $>1000.000$ unique visitors yearly: www.beleefdelente. $n \mathrm{l}$ )

Management Skills Training 2 ECTS

Organisation of seminars and courses

Wageningen Evolution and Ecology Seminars (WEES)

2014-2018

EDUCATION AND TRAINING TOTAL

71 ECTS $^{1}$

${ }^{1}$ One ECTS credit equals a study load of approximately 28 hours 


The research described in this thesis was financially supported by 'SEX SEL-VAR' project number 334544 funded by Marie Curie Career Integration Grant from the European Commission (FP7) and the Dr. J.L. Dobberke Foundation.

proefschriftmaken.nl II Digiforce

Cover Design by Judith van Voorst Vader

\section{Photo credits}

Irin-K / shutterstock.com

Maksimilian / shutterstock.com

Marion Wiercx 


\section{Propositions}

1. A universal Prince Charming does not exist.

(this thesis)

2. Mate preference and mate choice are separate processes, but only by considering both can we understand sexual selection.

(this thesis)

3. Unlike incorrect statistics, the concept of correct statistics is merely an opinion.

4. Fundamental science is fundamental to applied sciences.

5. Good work environments should facilitate individual differences in daily productivity patterns.

6. When communicating to the public about individual animal behaviour, we should never omit to educate them about the purpose of death and selection.

\section{Propositions belonging to the thesis, entitled:}

'A matter of taste: the role of compatibility in mate preferences in great tits'

Lies Zandberg

Wageningen, 2 November 2018 